\title{
Locoregional endpoints in breast cancer research
}

Citation for published version (APA):

Moossdorff, M. (2017). Locoregional endpoints in breast cancer research: Better, faster \& stronger results. [Doctoral Thesis, Maastricht University]. Maastricht University. https://doi.org/10.26481/dis.20171027mm

Document status and date:

Published: 01/01/2017

DOI:

10.26481/dis.20171027mm

Document Version:

Publisher's PDF, also known as Version of record

\section{Please check the document version of this publication:}

- A submitted manuscript is the version of the article upon submission and before peer-review. There can be important differences between the submitted version and the official published version of record.

People interested in the research are advised to contact the author for the final version of the publication, or visit the DOI to the publisher's website.

- The final author version and the galley proof are versions of the publication after peer review.

- The final published version features the final layout of the paper including the volume, issue and page numbers.

Link to publication

\footnotetext{
General rights rights.

- You may freely distribute the URL identifying the publication in the public portal. please follow below link for the End User Agreement:

www.umlib.nl/taverne-license

Take down policy

If you believe that this document breaches copyright please contact us at:

repository@maastrichtuniversity.nl

providing details and we will investigate your claim.
}

Copyright and moral rights for the publications made accessible in the public portal are retained by the authors and/or other copyright owners and it is a condition of accessing publications that users recognise and abide by the legal requirements associated with these

- Users may download and print one copy of any publication from the public portal for the purpose of private study or research.

- You may not further distribute the material or use it for any profit-making activity or commercial gain

If the publication is distributed under the terms of Article $25 \mathrm{fa}$ of the Dutch Copyright Act, indicated by the "Taverne" license above, 


\section{LOCOREGIONAL ENDPOINTS IN BREAST CANCER RESEARCH Better, faster \& stronger results}



Locoregional endpoints in breast cancer research:

better, faster \& stronger results 
(C) Martine Moossdorff, Maastricht 2017

No part of this book may be reproduced or transmitted in any form or by any means, without prior permission in writing by the author, or when appropriate, by the publishers of the publications.

Layout: $\quad$ Tiny Wouters

Cover image: Sabdesign85/Adobe Stock

Printing: ProefschriftMaken

ISBN/EAN: $\quad 9789462957459$ 


\title{
Locoregional endpoints in breast cancer research: better, faster \& stronger results
}

\author{
Proefschrift \\ ter verkrijging van de graad van doctor aan de Universiteit Maastricht, \\ op gezag van de Rector Magnificus, Prof dr. Rianne M. Letschert \\ volgens het besluit van het College van Decanen, \\ in het openbaar te verdedigen op \\ vrijdag 27 oktober 2017 om 10.00 uur
}

door

Martine Moossdorff 
Promotor

Prof. dr. L.P.S. Stassen

\section{Copromotores}

Dr. M.L. Smidt

Dr. L.J.A. Strobbe

Beoordelingscommissie

Prof. dr. F.C.S. Ramaekers (voorzitter)

Prof.dr. G.L. Beets

Prof.dr. R.M.A. Heeren

Dr. R.M.H. Roumen 


\section{Index}

$\begin{array}{ll}\text { I. General introduction and outline } & 7\end{array}$

II. Part 1 - Endpoint definitions in breast cancer research 13

Chapter 1. Inconsistent selection and definition of local and regional 15 endpoints in breast cancer research.

Chapter 2. Maastricht Delphi Consensus on event definitions for

classification of recurrence in breast cancer research.

Chapter 3. Contralateral lymph node recurrence in breast cancer: regional event rather than distant metastatic disease. A systematic review of the literature

Chapter 4. Prognosis of contralateral lymph node recurrence: data from national cancer registries and individual hospitals.

Chapter 5. TNM classification and the need for revision of pN3a breast cancer.

III Part 2 - Individual risk \& timing of recurrences during follow-up

Chapter 6. Local recurrence after mastectomy for breast cancer in the current era: which subgroups are still at risk?

Chapter 7. Conditional Local Recurrence: The effect of event-free years on the risk of 5-year local recurrence in different subtypes of breast cancer

Chapter 8. Conditional regional recurrence for different subtypes of breast cancer.

IV Summary, discussion and future perspectives

$\checkmark$ Appendices

Nederlandse samenvatting

Valorisation

Dankwoord

Curriculum vitae

List of publications 

General introduction and outline 



\section{General introduction and outline}

The enormous amount of breast cancer research that has been conducted over the past decades, has led to incredible improvements in survival and quality of life of breast cancer patients. For example, in the UK in 1971-72, the average age adjusted 5-year survival of any new diagnosed breast cancer patient (so all stages I-IV combined, including patients with distant metastases) was 52.2\%, whereas in 2010-11 this was 86.6\%. ${ }^{1}$ In The Netherlands, 5-year age adjusted survival has increased from $77 \%$ in 1989-1993 to $87 \%$ in 2008-2012. ${ }^{2}$ These results are spectacular and would not have been possible if thousands of researchers and millions of patients would not have dedicated their lives to these projects. This is of course, great news for breast cancer patients. However, there is still room for improvement in several aspects of the quality and efficiency of breast cancer research.

\section{Time and size: two problems in breast cancer research}

The spectacular improvement in survival is, besides great news for breast cancer patients, ironically also bad news for breast cancer research. In a way, breast cancer research is becoming the victim of its own success for two reasons. First, studies need to include more and more patients because the prognosis is favorable: because recurrence and death now fortunately occur in only few patients (especially in populations with early breast cancer), large numbers of patients are needed to produce reliable results, i.e. be sure the benefit of a treatment the study shows is not mere coincidence. In other words, large sample sizes are necessary to provide enough power. The second problem is that we need very long follow-up. Many breast cancer survivors live up to 10 to 20 years or even longer and although many recurrences occur in the first few years, it is known that breast cancer can recur many years after initial diagnosis. This has led to studies needing at least 5 but more often 10 years of follow-up before clinicians, insurance companies, governments, or other stakeholders are prepared to implement the results. If a woman enrolls in a breast cancer study today, and the new treatment proves to be superior, it may take over 10 to 15 years for that treatment to become standard of care.

Requiring very large numbers of patients and very long follow-up are two major problems in breast cancer research nowadays. They cause studies to be very expensive, as collecting, storing, and analyzing all the data from these patients is a very costly process. Critics already state that the proportion of attention and funds that are allocated to breast cancer research is too large, and other important diseases are neglected. ${ }^{3,4}$ Finding solutions for the required time and size of breast cancer studies would be a major step forward. 


\section{Outcome measurement in breast cancer research}

Another important issue in breast cancer research is whether the countless studies actually measure the same outcomes. Many different endpoints are used, such as for survival: examples are overall survival, disease-free survival, event-free survival, and breast cancer specific survival. The same goes for recurrence: examples include breast cancer recurrence, in-breast recurrence, local recurrence, ipsilateral breast tumor recurrence, locoregional relapse, regional recurrence, and distant metastasis. But do they all measure the same thing? If they don't, comparing them in reviews and guidelines, or pooling them in meta-analyses would be like comparing apples and oranges. Even an endpoint such as local recurrence consists of a set of events: breast cancer may recur in the same breast, in the other breast, in the skin or subcutaneous tissue, in the surgical scar, but some may also count the other breast or lymph nodes as local recurrences. This means that although "local recurrence" seems pretty straightforward, the definition may vary between studies. These inconsistencies limit mutual comparison of study results. In that way, inconsistent endpoint definitions may lead to incorrect conclusions and thus harm evidence-based treatment of breast cancer.

\section{Goal and outline}

It is our responsibility as doctors and researchers studying breast cancer to make sure we use the available funds and efforts optimally. We can improve that by carefully choosing both which outcome we measure and for how long we need to measure it. The aim of this thesis is to avoid comparing apples and oranges in breast cancer research to allow reliable comparison of results, and to explore if we can save research funds and decrease delay in implementation by investigating whether shorter follow-up time is also sufficient.

The first chapters are dedicated to differences in outcome measures: I will describe if breast cancer studies really use different endpoint definitions (Chapter 1), how we can make sure we use the same definitions in the future (Chapter 2) and whether there are events we should categorize differently (Chapters 3, 4, and 5). The second part of this thesis will focus on time: is it possible to tailor follow up to individual risk and to obtain results in less time (Chapters 6, 7, and 8)?

Finally, in the Summary, Discussion, and Future perspectives chapter, I will focus on the future of outcome measurement in breast cancer research, as well as interpretation and implementation in clinical practice. 


\section{References}

1 Cancer Research UK. Breast cancer survival statistics [Internet]. Cancer Research UK. 2015 [cited 2017 Jan 5]. Available from: http://www.cancerresearchuk.org/health-professional/cancer-statistics/statisticsby-cancer-type/breast-cancer/survival\#ref-2

2 IKNL. Cijfers over Kanker [Internet]. [cited 2017 Jan 5]. Available from: http://www.cijfersoverkanker.nl/selecties/dataset_1/img586e3d5d8a99d

3 Lung Cancer Alliance. 2012 Lung Cancer Facts [Internet]. 2012 [cited 2017 Jan 5]. Available from: http://www.lungcanceralliance.org/assets/docs/media/LCA\%20Funding\%20Fact\%20Sheet\%202012.pdf

4 Gander K. Why do some forms of cancer receive more research funding than others? [Internet]. The Independent. 2014 [cited 2017 Jan 5]. Available from: http://www.independent.co.uk/life-style/healthand-families/health-news/why-do-some-forms-of-cancer-receive-more-research-funding-than-others9771396.html 



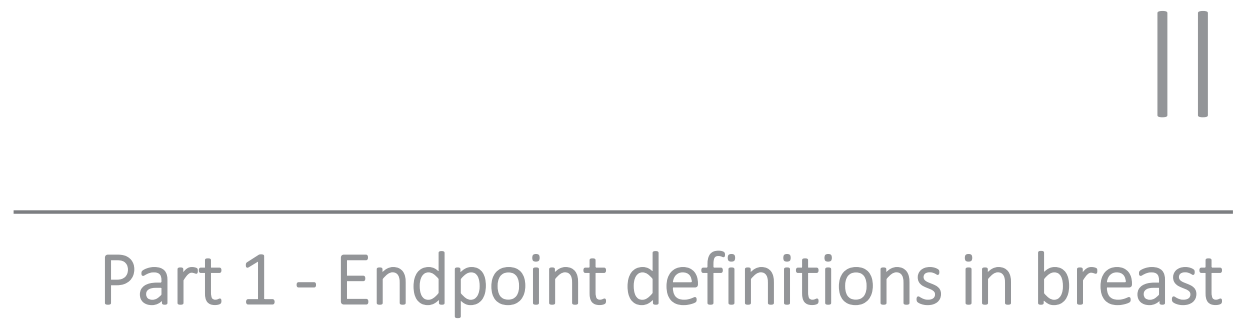

cancer research 



\section{Chapter 1}

Inconsistent selection and definition of local and regional endpoints in breast cancer research

Moossdorff M, van Roozendaal LM, Schipper RJ, Strobbe LJA, Voogd AC, Tjan-Heijnen VCG, Smidt ML * MM and LvR are co-first authors on this chapter British Journal of Surgery, 2014; 101: 1657-1665 


\section{Abstract}

\section{Background}

Results in breast cancer research are reported using study endpoints. Most are composite endpoints (such as locoregional recurrence), consisting of several components (for example local recurrence) that are in turn composed of specific events (such as skin recurrence). Inconsistent endpoint selection and definition might lead to unjustified conclusions when comparing study outcomes. This study aimed to determine which locoregional endpoints are used in breast cancer studies, and how these endpoints and their components are defined.

\section{Methods}

PubMed was searched for breast cancer studies published in nine leading journals in 2011. Articles using endpoints with a local or regional component were included and definitions were compared.

\section{Results}

Twenty-three different endpoints with a local or regional component were extracted from 44 articles. Most frequently used were disease-free survival (25 articles), recurrence-free survival (7), local control (4), locoregional recurrence-free survival (3) and event-free survival (3). Different endpoints were used for similar outcomes. Of 23 endpoints, five were not defined and 18 were defined only partially. Of these, 16 contained a local and 13 a regional component. Included events were not specified in 33 of 57 (local) and 27 of 50 (regional) cases. Definitions of local components inconsistently included carcinoma in situ and skin and chest wall recurrences. Regional components inconsistently included specific nodal sites and skin and chest wall recurrences.

\section{Conclusion}

Breast cancer studies use many different endpoints with a locoregional component. Definitions of endpoints and events are either not provided or vary between trials. To improve transparency, facilitate trial comparison and avoid unjustified conclusions, authors should report detailed definitions of all endpoints. 


\section{Introduction}

When comparing results of breast cancer studies, one is confronted with many different study endpoints and unclear definitions. Most studies have composite endpoints, for example locoregional recurrence, that consist of several components. These components consist of specific events, such as recurrence in axillary lymph nodes. Both the selection and definition of study endpoints (that is which specific events are included) may vary between studies. For instance, survival may be reported using a variety of endpoints, including disease-free survival, distant disease-free survival or breast cancer-specific survival. These endpoints do not always include the same components and events, and the paper may not provide the precise definition.

The definition of endpoints in breast cancer studies has been a topic of interest among medical researchers for several years. Cuzick $^{1}$ discussed inconsistent definitions of disease-free survival and noted that inconsistent selection of endpoints may confound the interpretation of study outcomes. Meropol ${ }^{2}$ advocated using a common language in cancer research outcome measures in general. Some efforts have been made to achieve uniform breast cancer endpoint definitions. Definitions for neoadjuvant and adjuvant trials were proposed by Hudis and colleagues ${ }^{3}$ in 2007 (Standardized Definitions for Efficacy End Points in adjuvant breast cancer trials, STEEP) and Fumagalli et al. ${ }^{4}$ in 2012. These definitions, however, have not been adopted universally into research practice. Since its publication in 2007, the STEEP article has been cited by 125 individual publications, according to PubMed Central, Google Scholar, Web of Knowledge and the Journal of Clinical Oncology website. A STEEP endpoint was used in 64 of these publications.

Comparing or pooling the results of studies using different endpoints, or the same endpoint with a different definition, may result in the comparison of apples and oranges. Therefore, comparing study results or pooling results in meta-analyses may not be justified, and may lead to incorrect conclusions. The aim of this study was to determine the extent of this problem, by providing an overview of local and regional study endpoints used in breast cancer studies, through a limited but representative review of the literature. The study explored which endpoints are being used, whether definitions are provided for the endpoints and their components, and, if so, which specific events are included. 


\section{Methods}

\section{Literature search}

The PubMed database was searched for experimental and observational research investigating breast cancer in humans. The PubMed limits 'clinical trials', 'randomized controlled trials' and 'comparative studies' were used, and the search was limited to research published between 1 January 2011 and 31 December 2011 in nine leading medical, surgical and radiation oncology journals. Journals were selected based on impact factor in order to provide an impression of study endpoints used in good-quality breast cancer research with considerable impact in the field.

Search terms were: breast neoplasms (MeSH), breast cancer, breast carcinoma, Annals of Surgery (Journal, NLM Catalog), Annals of Surgical Oncology (Journal), British Journal of Surgery (Journal), Journal of Clinical Oncology (Journal), Journal of the American Medical Association (Journal), Lancet (Journal), Lancet Oncology (Journal), New England Journal of Medicine (Journal) and Radiotherapy \& Oncology (Journal).

\section{Selection}

Articles found through this search were assessed for eligibility. Articles were subjected to review if the abstract met the following inclusion criteria: original research paper; observational or therapeutic study; investigation of any type of invasive early breast cancer; and use of a clinical study endpoint. Articles with study endpoints containing a local or regional component were analysed further. Selection of publications and endpoint extraction were performed independently by two authors. Discrepancies were resolved by consulting a third author.

\section{Data extraction}

All endpoints containing a local or regional component were extracted from the publications. Any definitions of the endpoints provided in the original article or appendix were extracted, including specific events included in the local and regional components.

\section{Results}

\section{Selection of articles}

The PubMed search identified 159 publications, of which 70 met the inclusion criteria. These 70 articles were evaluated for use of a local or regional study endpoint (or a 
composite endpoint with a local or regional component). This resulted in inclusion of 44 papers. A Preferred Reporting Items for Systematic Reviews and Meta-Analyses $(\text { PRISMA })^{5}$ flow chart is presented in Figure 1.1.

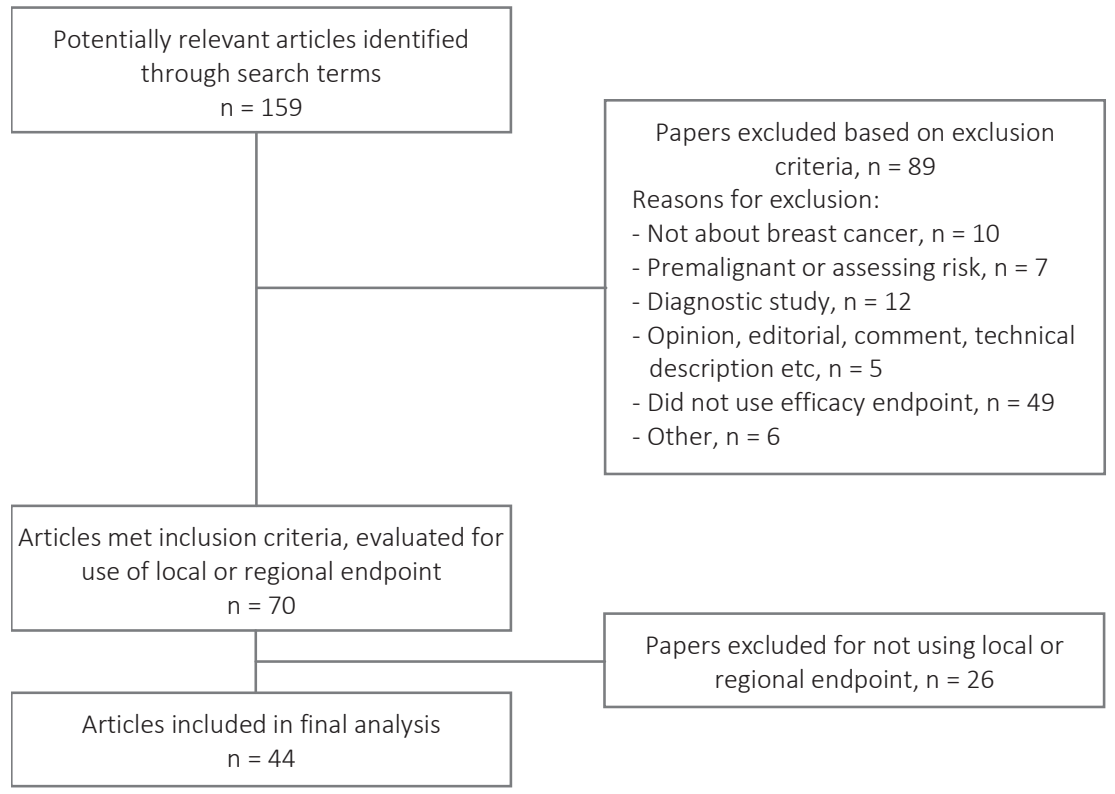

Figure 1.1 PRISMA flowchart: selection and inclusion of publications

\section{Local and regional study endpoints used in breast cancer trials}

The 44 articles $^{6-49}$ contained 23 different endpoints with a local and/or regional component (Table 1.1). Various study endpoints were used for similar outcomes. Of these 23 endpoints, disease-free survival was used most frequently (25 articles), followed by recurrence-free survival (7), local control (4), locoregional recurrence-free survival (3) and event-free survival (3). Twelve endpoints were used only once each among the 44 publications.

\section{Definitions of endpoints used}

Definitions of the endpoints were not provided consistently (Table 1.1). Five of 23 endpoints were not defined in any of the papers. The other 18 were defined partially at least once, describing either the time interval for a time-to-event endpoint (for 
example from randomization or from surgery) or describing which local and/or regional events were included, or both. Of the 18 defined endpoints, 16 contained a local component and 13 a regional component. Two endpoints were defined according to the STEEP guidelines ${ }^{3}$ : (invasive) breast cancer-free interval ${ }^{38}$ and invasive disease-free survival $^{13}$.

Table 1.1 Choice, frequency, and definitions of local and regional endpoints in 44 publications

\begin{tabular}{lcc}
\hline & No. of articles & $\begin{array}{c}\text { Definition provided } \\
\text { (at least partial) }\end{array}$ \\
\hline Local endpoints $(n=7)$ & 12 & 6 \\
Ipsilateral breast recurrence & 1 & 1 \\
Ipsilateral breast tumour recurrence & 2 & 1 \\
Ipsilateral breast relapse & 1 & 1 \\
Ipsilateral local tumour relapse & 1 & 1 \\
Local control & 4 & 1 \\
Local recurrence & 2 & 1 \\
Rate of cancer recurrence after mastectomy & 1 & 0 \\
Regional endpoints (n=3) & 4 & 3 \\
Axillary relapse & 1 & 1 \\
Crude cumulative incidence of axillary recurrence & 1 & 0 \\
Regional recurrence & 2 & 2 \\
Locoregional endpoints ( $n=3)$ & 6 & 4 \\
Local or regional failure & 1 & 0 \\
Locoregional control & 2 & 1 \\
Locoregional recurrence-free survival & 3 & 3 \\
Composite endpoints with local or regional component $(n=10)$ & 44 & 39 \\
Any breast cancer event & 1 & 0 \\
Breast cancer-free interval & 1 & 1 \\
Breast cancer-free survival & 1 & 1 \\
Disease-free survival & 25 & 25 \\
Event-free survival & 3 & 3 \\
Invasive disease-free survival & 1 & 1 \\
Recurrence-free survival & 7 & 6 \\
Relapse-free survival & 2 & 1 \\
Risk of recurrence & 1 & 0 \\
Time to recurrence & 2 & 52 \\
Total ( $n=23$ separate endpoints) & 66 & \\
\hline & & 1 \\
\hline
\end{tabular}

\section{Definitions of local components}

The 16 defined endpoints with a local component were used 57 times in the 44 articles (Table S1.1, supporting information). The definitions provided for these local components were compared with respect to the inclusion or exclusion of specific events. Events listed in definitions of the local component of endpoints included ipsilateral breast recurrence, in situ carcinomas, recurrence in skin, surgical scar and chest wall, and, in one case, lymph nodes. 
Over half of the cases (33 of 57) did not mention which specific events were included as a local recurrence. In the remaining 24, at least some included events were listed (Figure 1.2). Tumour recurrence in breast was included specifically 15 of 24 times. In contrast, the breast was not mentioned specifically six times. Three studies used an alternative definition for in-breast recurrence: one excluded resectable recurrences after lumpectomy and the other two subdivided breast recurrences as true/marginal or elsewhere in the breast. One of these papers also included recurrences in 'nodal basins' as a local event. None of the other papers made a distinction between true recurrences and new ipsilateral primary breast cancer. Carcinoma in situ was excluded as a local event eight times, but in the remaining 16 was neither included explicitly nor excluded. A skin recurrence was included twice as a local event, excluded once (but included as a regional recurrence) and not specified in the remaining 20 articles. One author included 'ipsilateral breast tissue and overlying skin'; no other author clarified whether the location of the skin recurrence (such as overlying tumour, in biopsy tract, or anywhere on the breast) was important. Recurrences in the surgical scar were included twice and not specified 22 times. Chest wall recurrences were mentioned as a local event in four articles, excluded once (but included as a regional recurrence) and unclear in the remaining 19 articles.

\section{Definitions of regional components}

Thirteen endpoints with a regional component were used 50 times in the 44 selected articles (Table S2, supporting information). Events listed under the regional components of these endpoints were skin and chest wall recurrences, as well as the involvement of lymph nodes in general and/or in specific nodal sites. In 27 of 50 cases, the articles did not specify the events that were considered regional recurrences. Fourteen of the remaining 23 cases included recurrences in 'lymph nodes' or 'nodal' recurrences, and nine described specific nodal sites that were included (Figure 1.3). These sites varied in the articles that provided this information; recurrences in axillary lymph nodes were specifically mentioned in nine, infraclavicular lymph nodes in two, supraclavicular lymph nodes in seven and internal mammary lymph nodes in seven. In six of the 23 cases, lymph nodes were not mentioned in the definition. In the remaining three, the endpoints disease-free survival, breast cancer-free survival and recurrence-free survival were said to include 'local or distant' recurrences, but did not refer to inclusion or exclusion of lymph node involvement. Of the 23 cases that listed the included and excluded events for the regional component, skin recurrences were included as a regional event in one, excluded in four and not specified in the remaining 18 . Chest wall recurrences were considered regional events in one of the 23 cases, excluded in six and unclear in 16. 

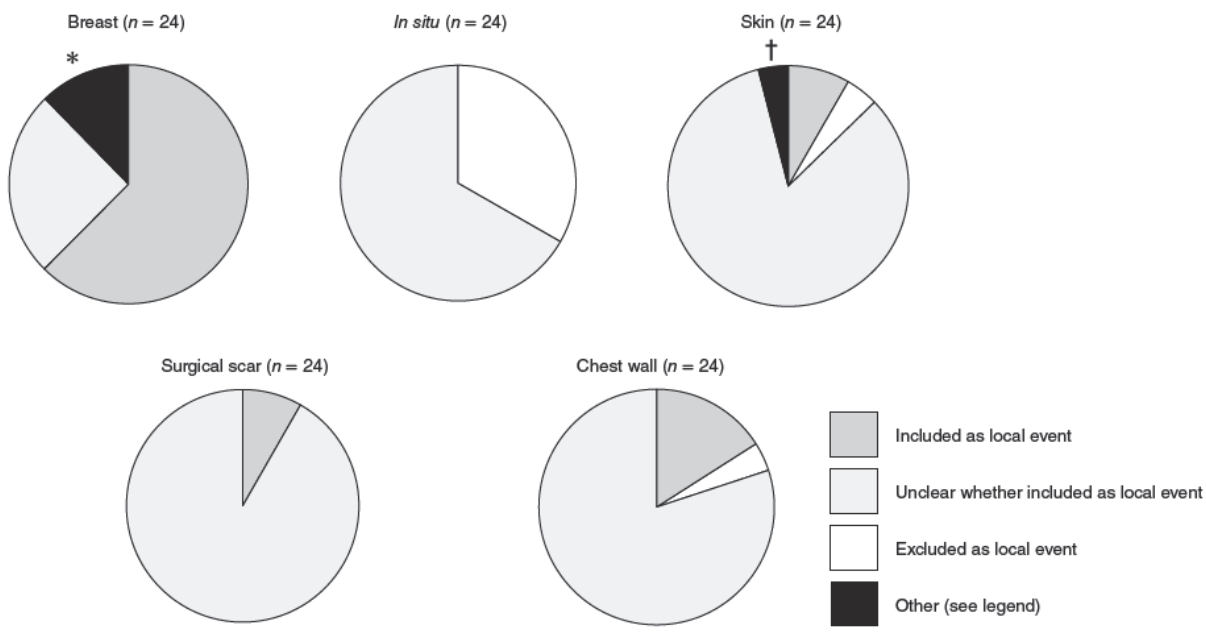

Figure 1.2 Events included as local recurrence in endpoints for which at least a partial definition was provided. *Distinguished true/marginal recurrence versus elsewhere in breast $(n=2)$ and excluded resectable recurrence after lumpectomy $(n=1) .+$ Ipsilateral breast tissue and overlying skin
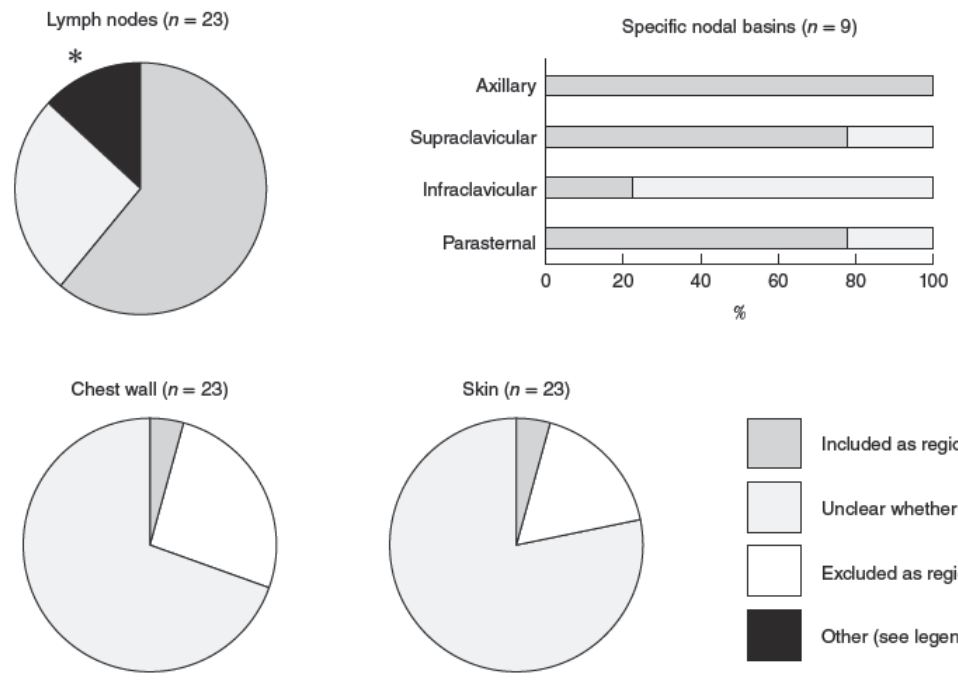

Included as regional event

Unclear whether included as regional event

Excluded as regional event

Other (see legend)

Figure 1.3 Events included as regional recurrence in endpoints for which at least a partial definition was provided. Included 'local and distant recurrence', without further mention of lymph nodes in disease-free survival, breast cancer-free survival and recurrence-free survival. * Recurrence in nodal basins was included once as a local recurrence 


\section{Discussion}

This study looked at breast cancer study endpoints with a local or regional component that were used in papers published in nine leading journals in 2011. There were several observations. First, many different endpoints were used for similar outcomes. Second, endpoint definitions were not provided consistently. For one in five endpoints, no definition at all could be deduced from the article; for others, definitions were often incomplete with respect to the specific events included or excluded as local or regional recurrences. Moreover, several inconsistencies in included local and regional events were observed between the definitions of similar endpoints. Only two of 44 papers used a standard definition of the endpoint.

Inconsistencies in the selection and definition of endpoints can limit interpretation and mutual comparison of trial results. Differences in study outcomes can be interpreted incorrectly as differences in treatment effects, leading to false conclusions and possible delays in the implementation of important study outcomes in clinical practice. Breast cancer studies are particularly vulnerable. Many new interventions show only small improvements in outcomes, considering the already favourable prognosis of most patients. ${ }^{50}$ When studying small absolute differences, the relative effect of varying endpoint definitions compared with the treatment effect may be even larger.

Almost all breast cancer study endpoints are composite endpoints. Composite endpoints have the advantage of increased event rates and, as a result, fewer patients are needed to provide significant results. However, for a composite endpoint to be a valid outcome measure, all included components, and subsequently the events included in these components, should meet predefined criteria. ${ }^{51}$ First, they should be of similar relevance to patients. If patients consider distant metastases and death to be of similar importance, it is not important how a risk reduction is distributed between the two. In contrast, ipsilateral ductal carcinoma in situ (DCIS) is less important to patients than mortality. In such instances, the distribution of risk reduction is important, and is not reflected properly if both are combined in one endpoint such as disease-free survival. Second, components should be influenced to a similar degree by the intervention. If an intervention effectively prevents breast recurrence but not distant metastasis, one endpoint measuring both does not provide specific information on treatment effect and may decrease the discriminative power of the study. The same applies to mortality, particularly in subgroups at high risk of non-breast cancer death, which is not influenced by the intervention to the same degree as breast cancer-specific mortality. Inclusion of all-cause mortality in an endpoint can therefore distort the results. ${ }^{52-54}$ Finally, the 
incidence of the more and less important components should be comparable. For instance, a high incidence of 'locoregional recurrence' could reflect either many lymph node recurrences and few instances of ipsilateral DCIS, or many cases of ipsilateral DCIS and few lymph node recurrences. In that case, the endpoint does not adequately reflect prognosis.

Clearly, not every endpoint currently used in breast cancer research meets these criteria. The standard definitions proposed by Hudis et al. $^{3}$ and Fumagalli and coworkers ${ }^{4}$ aimed to solve the problem of inconsistent use and definitions of study endpoints in adjuvant and neoadjuvant settings, but they are not used consistently. It is unknown why these definitions have not been adopted universally; possibilities include lack of awareness, the relatively short interval since publication, criticism of definitions, or anticipated problems in comparing new results with previous findings. For instance, the American College of Surgeons Oncology Group (ACOSOG) Z0011 study ${ }^{22}$ reported disease-free survival rather than protocol-specified distant disease-free survival to facilitate comparison with other studies. Additionally, these proposals focused on traditional adjuvant therapy trials, whereas the multidisciplinary character of breast cancer care requires easy comparison of results from other fields involved in management of breast cancer. An additional consensus-based proposal for standard definitions of endpoints in cancer research, including breast cancer, might be expected from the Definition for the Assessment of Time-to-event Endpoints in CANcer trials (DATECAN)group. ${ }^{55}$

The detrimental effect of inconsistent endpoint definitions on reliable comparison of trial results may be even larger when different events occur in the same patient. In patients with synchronous distant metastasis and axillary recurrence, researchers may only count distant metastasis and ignore the axillary recurrence, or count distant metastasis and include the axillary recurrence separately in an analysis of locoregional control. As the chosen approach either increases or decreases the event rate, differences between trials may contribute to variations in reported study outcomes. The same applies to the question of whether a thorough search for synchronous locoregional events should be conducted once distant metastases have occurred. These issues should be taken into account when interpreting trial results and again stress the need for a standard approach.

The articles selected for this review were published in only nine journals over a relatively short time. Furthermore, only endpoints with a local and/or regional component were selected. Therefore, the list of endpoints and variable definitions is probably not 
exhaustive, which may limit extrapolation of the results. With a longer time frame and additional journals, even more different endpoints and definitions could be encountered. It is striking, therefore, that such a large variety of endpoints was identified even in this limited search and that definitions were not provided consistently. Furthermore, many different definitions were used for similar endpoints. Additionally, it was found that the lack of definition of local and regional events lies at the root of inconsistent endpoint definitions. These inconsistencies suggest that detailed endpoint definitions do not have the full attention of authors and reviewers. It is unlikely that this problem is limited to the selection of journals or time frame of the search. Therefore, despite these restrictions, the results illustrate that the outcomes of major breast cancer studies are not readily comparable as a result of inconsistencies in endpoint selection and definition.

To improve transparency, facilitate trial comparison and avoid unjustified conclusions, authors should provide clear and detailed definitions of the endpoints. Preferably, standard endpoint definitions should be used, to facilitate reliable comparison of results. This also applies to definitions of the components included in the endpoints, such as local and regional events. To ensure transparency in endpoint definitions, clinical trial registries, reviewers of research protocols and journals publishing the results should insist on inclusion of detailed definitions of endpoints and their components. These should comprise at least all included (and excluded) events and, for time-to-event endpoints, the starting point (for example from randomization, from surgery). The Consolidated Standards of Reporting Trials (CONSORT) and STrengthening the Reporting of OBservational studies in Epidemiology (STROBE) checklists already contain an item requiring listing and definition of endpoints. Subsequently, journal editors and reviewers should assess whether these are covered sufficiently.

Designing standard endpoints for breast cancer trials should start with standard definitions of the specific components of these endpoints, such as local or regional recurrence. Only when the definitions of these components are used consistently can a valid and relevant combination be chosen as a valid and relevant composite endpoint. Currently, a consensus project using the RAND/UCLA Appropriateness Method, ${ }^{56}$ an adjusted version of the Delphi method, aiming to reach consensus on the definitions of local event, second primary breast cancer, regional event and distant event, is being undertaken. An international expert panel was formed for this purpose, consisting of leading breast cancer specialists, epidemiologists, presidents and members of scientific and clinical societies and boards, research groups, and editors and editorial board members of leading cancer journals. The proposed event definitions can be used to 
improve existing standard endpoint definitions or, if necessary, to build further towards a new proposal.

In anticipation of these proposals, authors reporting trial results should improve transparency in two ways. First, definitions of all endpoints and their components must be provided in the paper, so any differences in definitions between trials become evident. Second, authors should report the incidence of all separate events in a supplement, in addition to the incidence of the endpoint. For instance, a trial using 'locoregional recurrence' as the primary endpoint should also provide the incidence of all included events, such as ipsilateral recurrence in the breast, skin recurrence and recurrence in a supraclavicular lymph node. This improves transparency even further, and may help interpret conflicting results. As a result of these improvements, more reliable conclusions will become available, serving patients with breast cancer worldwide. 


\section{References}

1. Cuzick J. Controversies in design and interpretation of adjuvant clinical trials. Cancer Invest 2010; 28(Suppl 1):28-34.

2. Meropol NJ. Comparative effectiveness research to inform medical decisions: the need for common language. J Clin Oncol 2012;30:4192-4193.

3. Hudis CA, Barlow WE, Costantino JP, Gray RJ, Pritchard KI, Chapman JA et al. Proposal for standardized definitions for efficacy end points in adjuvant breast cancer trials: the STEEP system. J Clin Oncol 2007; 25:2127-2132.

4. Fumagalli D, Bedard PL, Nahleh Z, Michiels S, Sotiriou C, Loi S et al. A common language in neoadjuvant breast cancer clinical trials: proposals for standard definitions and endpoints. Lancet Oncol 2012;13: e240-e248.

5. Moher D, Liberati A, Tetzlaff J, Altman DG, Group P. Preferred reporting items for systematic reviews and meta-analyses: the PRISMA statement. PLoS Med 2009;6:e1000097.

6. Abdulkarim BS, Cuartero J, Hanson J, Deschênes J, Lesniak D, Sabri S. Increased risk of locoregional recurrence for women with T1-2NO triple-negative breast cancer treated with modified radical mastectomy without adjuvant radiation therapy compared with breast-conserving therapy. J Clin Oncol 2011;29:2852-2858.

7. Adkins FC, Gonzalez-Angulo AM, Lei X, Hernandez-Aya LF, Mittendorf EA, Litton JK et al. Triple-negative breast cancer is not a contraindication for breast conservation. Ann Surg Oncol 2011;18:3164-3173.

8. Agrawal RK, Alhasso A, Barrett-Lee PJ, Bliss JM, Bliss P et al.; FAST Trialists group. First results of the randomized UK FAST Trial of radiotherapy hypofractionation for treatment of early breast cancer (CRUKE/04/015). Radiother Oncol 2011;100:93-100.

9. Badwe R, Hawaldar R, Parmar V, Nadkarni M, Shet T, Desai S et al. Single-injection depot progesterone before surgery and survival in women with operable breast cancer: a randomized controlled trial. J Clin Oncol 2011;29:2845-2851.

10. Bartlett JM, Brookes CL, Robson T, van de Velde CJ, Billingham L, Campbell FM et al. Estrogen receptor and progesterone receptor as predictive biomarkers of response to endocrine therapy: a prospectively powered pathology study in the Tamoxifen and Exemestane Adjuvant Multinational trial. J Clin Oncol 2011;29:1531-1538.

11. Bliss JM, Kilburn LS, Coleman RE, Forbes JF, Coates AS, Jones SE et al. Disease-related outcomes with long-term follow-up: an updated analysis of the intergroup exemestane study. J Clin Oncol 2012;30: 709-717.

12. Bollet MA, Belin L, Reyal F, Campana F, Dendale R, Kirova YM et al. Preoperative radio-chemotherapy in early breast cancer patients: long-term results of a phase II trial. Radiother Oncol 2012;102:82-88.

13. Coleman RE, Marshall H, Cameron D, Dodwell D, Burkinshaw R, Keane M et al. Breast-cancer adjuvant therapy with zoledronic acid. N Engl J Med 2011;365:1396-1405.

14. Colleoni M, Giobbie-Hurder A, Regan MM, Thürlimann B, Mouridsen H, Mauriac L et al. Analyses adjusting for selective crossover show improved overall survival with adjuvant letrozole compared with tamoxifen in the BIG 1-98 study. J Clin Oncol 2011;29:1117-1124.

15. Coombes RC, Bliss JM, Espie M, Erdkamp F,Wals J, Tres A et al. Randomized, phase III trial of sequential epirubicinand docetaxel versus epirubicin alone in postmenopausal patients with node-positive breast cancer. J Clin Oncol 2011;29:3247-3254.

16. EiermannW, Pienkowski T, Crown J, Sadeghi S, Martin M, Chan A et al. Phase III study of doxorubicin/cyclophosphamide with concomitant versus sequential docetaxel as adjuvant treatment in patients with human epidermal growth factor receptor 2-normal, node-positive breast cancer: BCIRG005 trial. J Clin Oncol 2011;29:3877-3884.

17. Elkin EB, Klem ML, Gonzales AM, Ishill NM, Hodgson D, Ng AK et al. Characteristics and outcomes of breast cancer in women with and without a history of radiation for Hodgkin's lymphoma: a multiinstitutional, matched cohort study. J Clin Oncol 2011;29:2466-2473. 
18. Ellis GK, Barlow WE, Gralow JR, Hortobagyi GN, Russell CA, Royce ME et al. Phase III comparison of standard doxorubicin and cyclophosphamide versus weekly doxorubicin and daily oral cyclophosphamide plus granulocyte colony-stimulating factor as neoadjuvant therapy for inflammatory and locally advanced breast cancer: SWOG 0012. J Clin Oncol 2011;29: 1014-1021.

19. Fortunato L, Mascaro A, Poccia I, Andrich R, Amini M, Costarelli L et al. Lobular breast cancer: same survival and local control compared with ductal cancer, but should both be treated the same way? Analysis of an institutional database over a 10-year period. Ann Surg Oncol 2012;19:1107-1114.

20. Gentilini O, Veronesi P, Botteri E, Soggiu F, Trifirò G, Lissidini G et al. Sentinel lymph node biopsy in multicentric breast cancer: five-year results in a large series from a single institution. Ann Surg Oncol 2011;18:2879-2884.

21. Gianni L, Dafni U, Gelber RD, Azambuja E, Muehlbauer S, Goldhirsch A et al. Treatment with trastuzumab for 1 year after adjuvant chemotherapy in patients with HER2-positive early breast cancer: a 4-year follow-up of a randomized controlled trial. Lancet Oncol 2011;12:236-244.

22. Giuliano AE, Hunt KK, Ballman KV, Beitsch PD, Whitworth PW, Blumencranz PWet al. Axillary dissection vs no axillary dissection in women with invasive breast cancer and sentinel node metastasis: a randomized clinical trial. JAMA 2011;305:569-575.

23. Gnant M, Mlineritsch B, Stoeger H, Luschin-Ebengreuth G, Heck D, Menzel C et al. Adjuvant endocrine therapy plus zoledronic acid in premenopausal women with early-stage breast cancer: 62-month followup from the ABCSG-12 randomised trial. Lancet Oncol 2011;12:631-641.

24. Gobardhan PD, Elias SG, Madsen EV, vanWely B, van den Wildenberg F, Theunissen EB et al. Prognostic value of lymph node micrometastases in breast cancer: a multicenter cohort study. Ann Surg Oncol 2011;18:1657-1664.

25. Hackshaw A, Roughton M, Forsyth S, Monson K, Reczko K, Sainsbury R et al. Long-term benefits of 5 years of tamoxifen: 10-year follow-up of a large randomized trial in women at least 50 years of age with early breast cancer. J Clin Oncol 2011;29:1657-1663.

26. Isern AE, Manjer J, Malina J, Loman N, Mårtensson T, Bofin A et al. Risk of recurrence following delayed large flap reconstruction after mastectomy for breast cancer. Br J Surg 2011;98:659-666.

27. Jin H, Tu D, Zhao N, Shepherd LE, Goss PE. Longer-term outcomes of letrozole versus placebo after 5 years of tamoxifen in the NCIC CTGMA.17 trial: analyses adjusting for treatment crossover. J Clin Oncol 2012;30:718-721.

28. Joensuu H, Kellokumpu-Lehtinen PL, Huovinen R, Jukkola-Vuorinen A, Tanner M, Kokko R et al. Adjuvant capecitabine, docetaxel, cyclophosphamide, and epirubicin for early breast cancer: final analysis of the randomized FinXX trial. J Clin Oncol 2012;30:11-18.

29. Kauer-Dorner D, Pötter R, Resch A, Handl-Zeller L,Kirchheiner K, Meyer-Schell K et al. Partial breast irradiation for locally recurrent breast cancer within a second breast conserving treatment: alternative to mastectomy? Results from a prospective trial. Radiother Oncol 2012;102:96-101.

30. Kimple RJ, Klauber-DeMore N, Kuzmiak CM, Pavic D, Lian J, Livasy CA et al. Local control following singledose intraoperative radiotherapy prior to surgical excision of early-stage breast cancer. Ann Surg Oncol 2011;18:939-945.

31. Li S, Yu KD, Fan L, Hou YF, Shao ZM. Predicting breast cancer recurrence following breast-conserving therapy: a single-institution analysis consisting of 764 Chinese breast cancer cases. Ann Surg Oncol 2011; 18:2492-2499.

32. Lipton A, Chapman JA, Demers L, Shepherd LE, Han L,Wilson CF et al. Elevated bone turnover predicts for bone metastasis in postmenopausal breast cancer: results of NCIC CTG MA.14. J Clin Oncol 2011;29: 3605-3610.

33. Martelli G, Miceli R, Daidone MG, Vetrella G, Cerrotta AM, Piromalli D et al. Axillary dissection versus no axillary dissection in elderly patients with breast cancer and no palpable axillary nodes: results after 15 years of follow-up. Ann Surg Oncol 2011;18:125-133.

34. Perez EA, Romond EH, Suman VJ, Jeong JH, Davidson NE, Geyer CE Jr et al. Four-year follow-up of trastuzumab plus adjuvant chemotherapy for operable human epidermal growth factor receptor 2positive breast cancer: joint analysis of data from NCCTG N9831 and NSABP B-31. J Clin Oncol 2011;29: 3366-3373.

35. Perez EA, Suman VJ, Davidson NE, Gralow JR, Kaufman PA, Visscher DW et al. Sequential versus concurrent trastuzumab in adjuvant chemotherapy for breast cancer. J Clin Oncol 2011;29:4491-4497. 
36. Pfeiler G, Königsberg R, Fesl C, Mlineritsch B, Stoeger $H$, Singer CF et al. Impact of body mass index on the efficacy of endocrine therapy in premenopausal patients with breast cancer: an analysis of the prospective ABCSG-12 trial. J Clin Oncol 2011;29:2653-2659.

37 Pritchard KI, Shepherd LE, Chapman JA, Norris BD, Cantin J, Goss PE et al. Randomized trial of tamoxifen versus combined tamoxifen and octreotide LAR therapy in the adjuvant treatment of early-stage breast cancer in postmenopausal women: NCIC CTG MA.14. J Clin Oncol 2011;29:3869-3876.

38. Regan MM, Neven P, Giobbie-Hurder A, Goldhirsch A, Ejlertsen B, Mauriac L et al. Assessment of letrozole and tamoxifen alone and in sequence for postmenopausal women with steroid hormone receptor-positive breast cancer: the BIG 1-98 randomised clinical trial at $8 \cdot 1$ years median follow-up. Lancet Oncol 2011;12:1101-1108.

39. Shah C, Antonucci JV,Wilkinson JB,Wallace M, Ghilezan M, Chen P et al. Twelve-year clinical outcomes and patterns of failure with accelerated partial breast irradiation versus whole-breast irradiation: results of a matched-pair analysis. Radiother Oncol 2011;100:210-214.

40. Slamon D, EiermannW, Robert N, Pienkowski T, Martin M, Press M et al. Adjuvant trastuzumab in HER2positive breast cancer. N Engl J Med 2011;365:1273-1283.

41. Stull TS, Catherine Goodwin M, Gracely EJ, Chernick MR, Carella RJ, Frazier TG et al. A single-institution review of accelerated partial breast irradiation in patients considered "cautionary" by the American Society for Radiation Oncology. Ann Surg Oncol 2012;19:553-559.

42. Tausch C, Taucher S, Dubsky P, Seifert M, Reitsamer R, KwasnyWet al. Prognostic value of number of removed lymph nodes, number of involved lymph nodes, and lymph node ratio in 7502 breast cancer patients enrolled onto trials of the Austrian Breast and Colorectal Cancer Study Group (ABCSG). Ann Surg Oncol 2012;19:1808-1817.

43. Untch M, Fasching PA, Konecny GE, Hasmüller S, Lebeau A, Kreienberg R et al. Pathologic complete response after neoadjuvant chemotherapy plus trastuzumab predicts favorable survival in human epidermal growth factor receptor 2-overexpressing breast cancer: results from the TECHNO trial of the AGO and GBG study groups. J Clin Oncol 2011;29:3351-3357.

44. van de Velde CJ, Rea D, Seynaeve C, Putter H, Hasenburg A, Vannetzel JM et al. Adjuvant tamoxifen and exemestane in early breast cancer (TEAM): a randomised phase 3 trial. Lancet 2011;377:321-331.

45. Wang J, Shi M, Ling R, Xia Y, Luo S, Fu X et al. Adjuvant chemotherapy and radiotherapy in triple-negative breast carcinoma: a prospective randomized controlled multi-center trial. Radiother Oncol 2011;100: 200-204.

46. Weaver DL, Ashikaga T, Krag DN, Skelly JM, Anderson SJ, Harlow SP et al. Effect of occult metastases on survival in node-negative breast cancer. N Engl J Med 2011;364:412-421.

47. Wennemers M, Bussink J, Grebenchtchikov N, Sweep FC, Span PN. TRIB3 protein denotes a good prognosis in breast cancer patients and is associated with hypoxia sensitivity. Radiother Oncol 2011;101: 198-202.

48. Werkhoven E, Hart G, Tinteren H, Elkhuizen P, Collette L, Poortmans $P$ et al. Nomogram to predict ipsilateral breast relapse based on pathology review from the EORTC 22881-10882 boost versus no boost trial. Radiother Oncol 2011;100:101-107.

49. Wink CJ,Woensdregt K, Nieuwenhuijzen GA, van der Sangen MJ, Hutschemaekers S, Roukema JA et al. Hormone treatment without surgery for patients aged 75 years or older with operable breast cancer. Ann Surg Oncol 2012;19:1185-1191.

50. Newman LA. Epidemiology of locally advanced breast cancer. Semin Radiat Oncol 2009;19:195-203.

51. Montori VM, Permanyer-Miralda G, Ferreira-Gonzalez I, Busse JW, Pacheco-Huergo V, Bryant D et al. Validity of composite end points in clinical trials. BMJ 2005;330:594-596.

52. Nout RA, Fiets WE, Struikmans H, Rosendaal FR, Putter H, Nortier JW. The in- or exclusion of non-breast cancer related death and contralateral breast cancer significantly affects estimated outcome probability in early breast cancer. Breast Cancer Res Treat 2008;109:567-572.

53. Mell LK, Jeong J, Nichols MA, Polite BN, Weichselbaum RR, Chmura SJ. Predictors of competing mortality in early breast cancer. Cancer 2010;116:5365-5373.

54. Mell LK, Jeong JH. Pitfalls of using composite primary end points in the presence of competing risks. J Clin Oncol 2010;28:4297-4299. 
55. Bellera CA, Pulido M, Gourgou S, Collette L, Doussau A, Kramar A et al. Protocol of the Definition for the Assessment of Time-to-event Endpoints in CANcer trials (DATECAN) project: formal consensus method for the development of guidelines for standardised time-to-event endpoints' definitions in cancer clinical trials. Eur J Cancer 2013;49:769-781.

56. Fitch K, Bernstein SJ, Aguilar MS, Burn B, LaCalle JR, Lazaro P et al. The RAND/UCLA Appropriateness Method User's Manual. RAND: Santa Monica, 2001. 


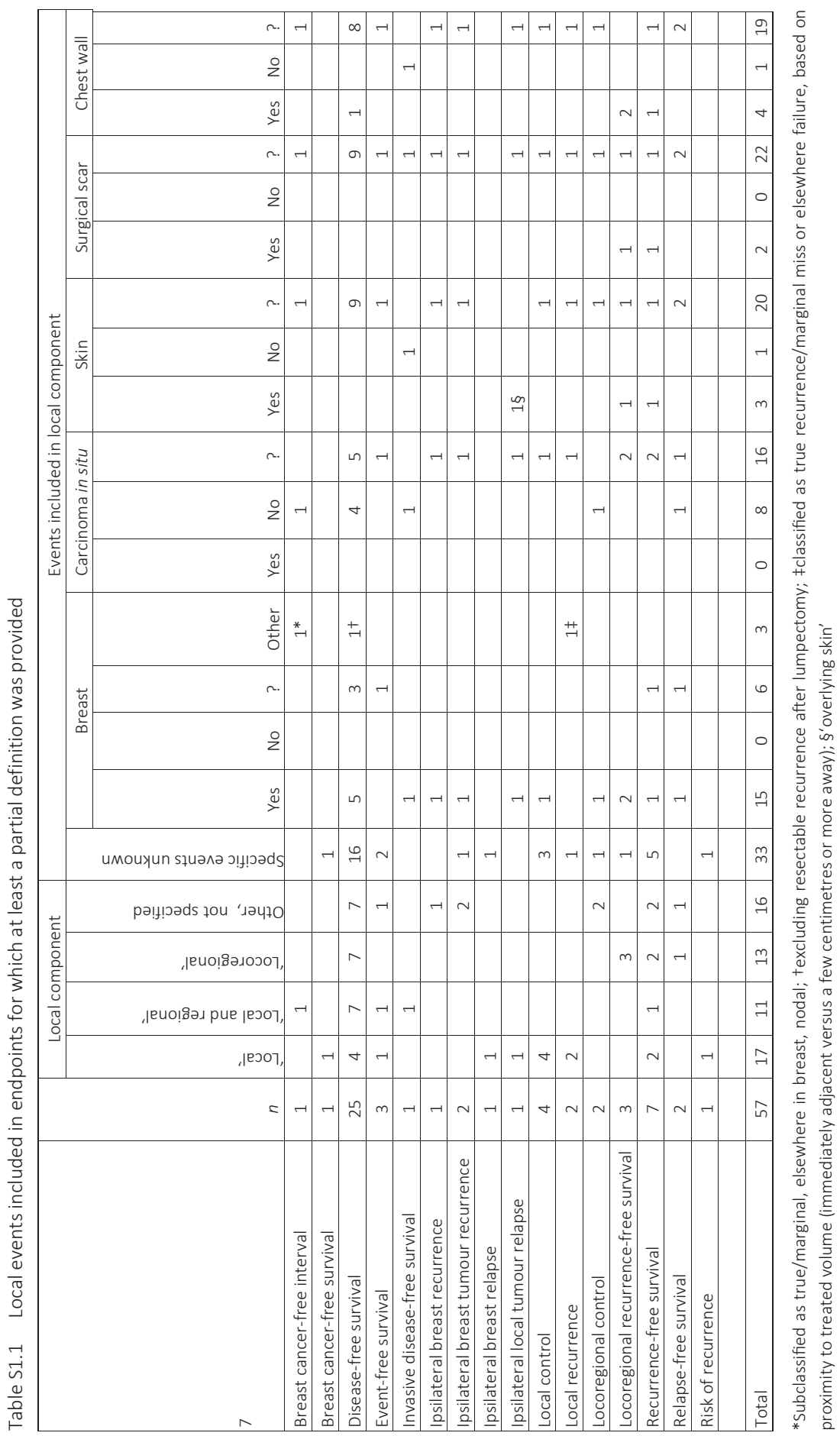




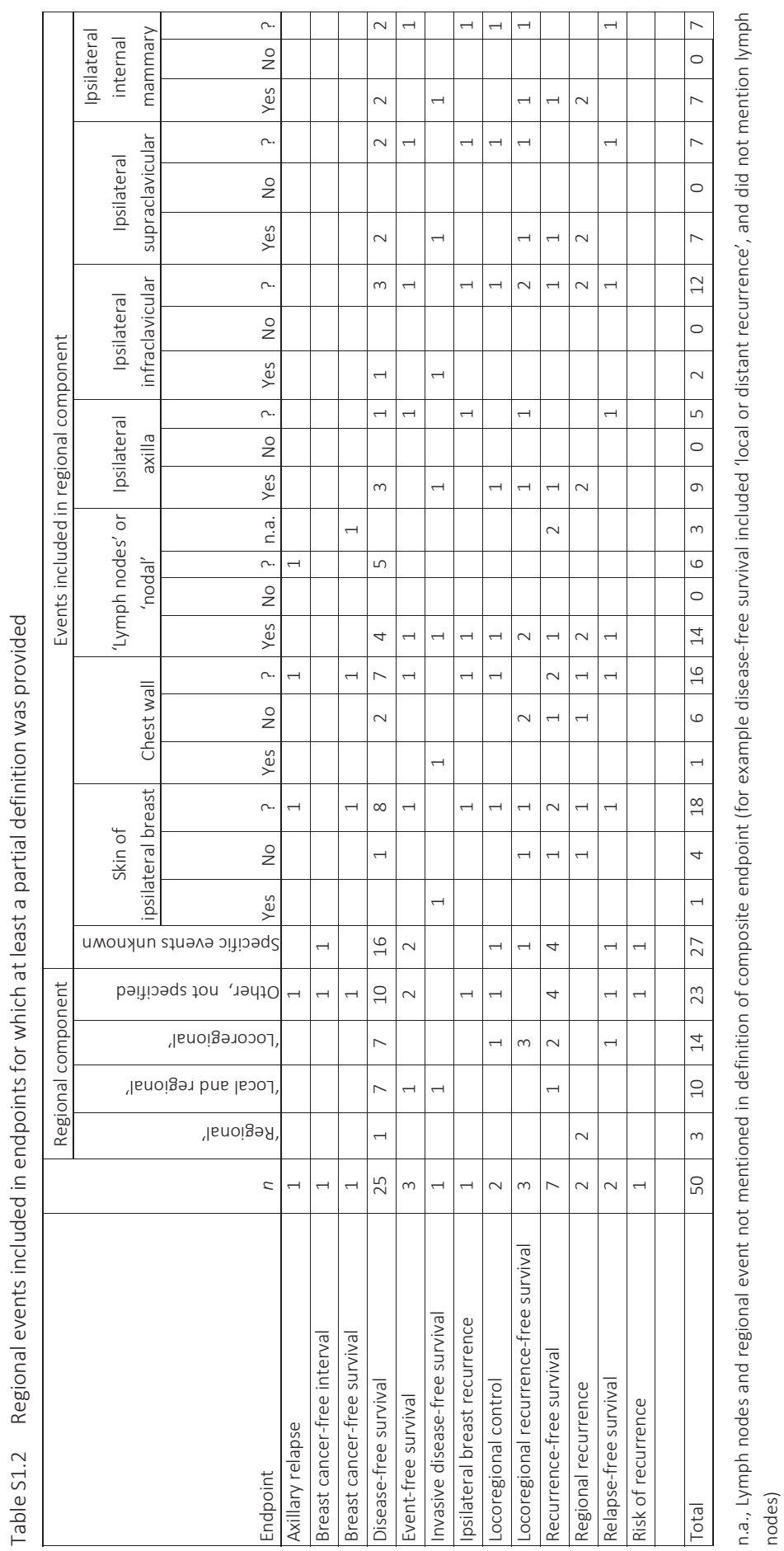




\section{Chapter 2}

\section{Maastricht Delphi Consensus on event definitions for classification of recurrence in}

breast cancer research

Moossdorff M, Van Roozendaal LM, Strobbe LJA, Aebi S, Cameron DA, Dixon JM, Giuliano AE, Haffty BG, Hudis CA, Klimberg S, Koczwara B, Kühn T, Lippman ME, Lucci A, Piccart MJ, Smith BD, Tjan-Heijnen VCG, Van de Velde CJH, Van Zee KJ, Vermorken JB, Viale G, Voogd AC, Wapnir IL, White J, Smidt ML JNCl J Natl Cancer Inst (2014) 106(12): dju288 


\section{Abstract}

\section{Background}

In breast cancer studies, many different endpoints are used. Definitions are often not provided or vary between studies. For instance, "local recurrence" may include different components in similar studies. This limits transparency and comparability of results. This project aimed to reach consensus on the definitions of local event, second primary breast cancer, regional and distant event for breast cancer studies.

\section{Methods}

The RAND-UCLA Appropriateness method (modified Delphi method) was used. A Consensus Group of international breast cancer experts was formed, including representatives of all involved clinical disciplines. Consensus was reached in two rounds of online questionnaires and one meeting.

\section{Results}

Twenty-four international breast cancer experts participated. Consensus was reached on 134 items in four categories. Local event is defined as any epithelial breast cancer or ductal carcinoma in situ (DCIS) in the ipsilateral breast, or skin and subcutaneous tissue on the ipsilateral thoracic wall. Second primary breast cancer is defined as epithelial breast cancer in the contralateral breast. Regional events are breast cancer in ipsilateral lymph nodes. A distant event is breast cancer in any other location. Therefore, this includes metastasis in contralateral lymph nodes and breast cancer involving the sternal bone. If feasible, tissue sampling of a first, solitary, lesion suspected for metastasis is highly recommended.

\section{Conclusion}

This project resulted in consensus-based event definitions for classification of recurrence in breast cancer research. Future breast cancer research projects should adopt these definitions to increase transparency. This should facilitate comparison of results and conducting reviews as well as meta-analysis. 


\section{Introduction}

When reporting breast cancer outcomes, many different endpoints are used. Definitions of these endpoints are not consistently provided and vary between trials. ${ }^{1}$ These inconsistencies limit transparency and comparison of study results. For instance, when interpreting different trials, it is important to know if "breast cancer-free interval" and "disease-specific survival" can be readily compared. Furthermore, even if studies use the same endpoint terminology, these endpoints may not include the same events. An endpoint such as "disease-free survival" may include local, regional, and distant events, as well as mortality and second primary cancer. Even if an endpoint consists of the same events (such as local recurrence), the specific components (eg, breast cancer in skin, metastasis in contralateral lymph node) included in these events may also vary. Therefore, the lack of consistent definition of events lies at the very root of the problem of inconsistent endpoint definitions.

These inconsistencies may compromise transparency of results. Differences in the reported outcome may reflect inconsistent end- point definitions, rather than treatment effect. This is especially the case when the absolute number of events is low, such as in early breast cancer. When the absolute number of events is small, adding or omit- ting a component (e.g., ipsilateral LCIS to local event) will have a proportionally larger effect on the incidence of the reported outcome. Therefore, there is need for standardized definitions of end- points. Several authors have addressed this problem. ${ }^{1-4}$ Efforts have been made to achieve uniform endpoint definitions in breast cancer research, specifically for the neoadjuvant and adjuvant setting. ${ }^{5,6}$ Such proposals are important steps towards overcoming this problem. Ideally, definitions are based on evidence regarding incidence, prognostic and therapeutic consequences, importance to patients, and degree to which the component is influenced by the intervention. ${ }^{7}$ However, for many events in breast cancer research, solid evidence regarding these criteria is not available. Therefore, expert consensus is a suitable alternative.

The aim of this project was to achieve consensus on the definitions of the most commonly used components in breast cancer study endpoints: local event, second primary breast cancer, regional event, and distant event, in order to improve transparency and facilitate comparison of results. 


\section{Methods}

The RAND/UCLA Appropriateness Method ${ }^{8}$ was used to assess consensus in an expert panel on the definitions of local event, second primary breast cancer, regional event, and distant event.

\section{Consensus methods}

Several formal consensus methods are available.,10 Among these is the Delphi method, which was introduced in the 1950s for decision making and forecasting for military purposes. $^{11}$ In a Delphi study, several rounds of questionnaires are completed by an expert panel. The aim is convergence of opinions as the process advances, by allowing panel members to adapt their opinions based on input from the panel. This is done anonymously, to minimize the influence of seniority, presumptions of expertise, and dominant characters. Since the introduction, the Delphi method has been used and adapted many times. One of those adaptions is the RAND/UCLA Appropriateness Method (RAM), ${ }^{8}$ often used for medical research. The RAM constitutes of a number of questionnaires followed by a face-to-face meeting to address unresolved disagreement.

\section{Steps of the consensus process}

The consensus process is summarized in Figure 2.1. First, a limited review of the literature was performed to assess which items may be included as local events, second primary breast cancers, regional events, and distant events.

Second, breast cancer experts were contacted personally by email to assess their willingness to participate. Potential panel members were selected based on considerable experience with high impact breast cancer research (surgical treatment, radiotherapy, [neo]adjuvant systemic therapy, prognostic, and epidemiological studies), occupation of leading positions on professional boards and societies, leading positions in major breast cancer research groups, and/or leading positions in major journals. In addition, the aim was to create a balanced panel in terms of discipline, geography, gender, and affiliation to major research groups and professional organizations.

Third, the questionnaires were developed and distributed using SurveyMonkey (SurveyMonkey, Inc., Palo Alto, CA; www.surveymonkey.com). The list of items was based on the literature review, as well as suggestions from breast cancer experts. Panel members were asked to score on a nine-point scale whether they found it appropriate to include the specific item as a local event, second primary breast cancer, regional event, and distant event. No open questions were asked. Participants were encouraged 
to list additional items and other important factors in free text fields after each question. An example question is shown in Figure 2.2.

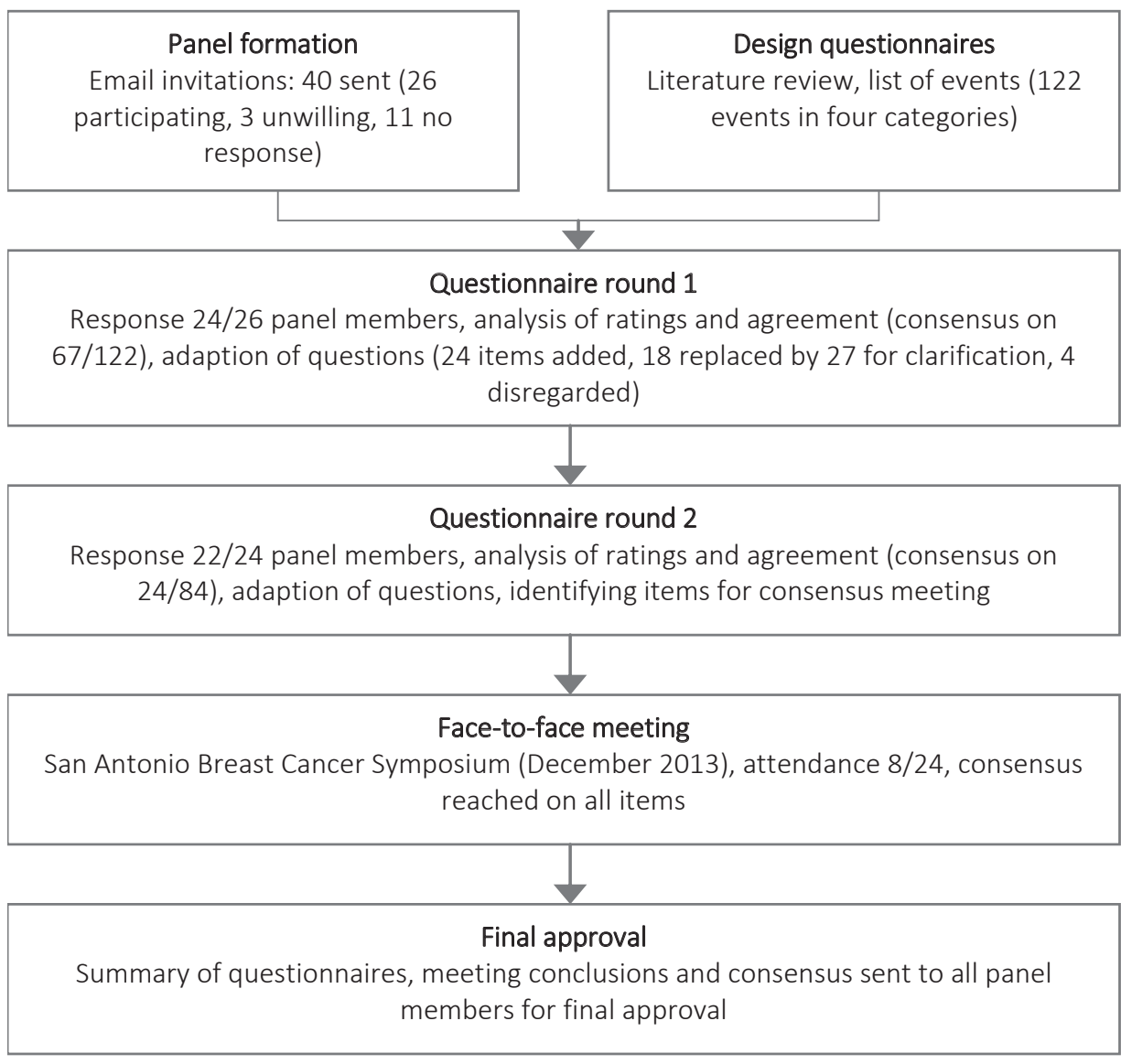

Figure 2.1 Flow-chart of the consensus process

The second questionnaire was based on the first. Items on which consensus was reached were not repeated. Items that were unclear or ambiguous based on comments in the free text fields were adjusted and repeated. Items suggested by panel members were added. For repeated items, the median and range of the ratings, as well as any additional remarks were provided. Consequently, arguments for rating the item were available to other panel members in the second round and meeting. The results of the second questionnaire were analyzed as described above. 
A face-to-face meeting was held during the San Antonio Breast Cancer Symposium in December 2013 to resolve any remaining issues. Panel members who completed the first survey were invited. After introduction of the item with presentation of the median rating, range, and any additional remarks, the item was discussed. After the discussion, panel members rated the item again on a nine-point scale. This lead resulted either in agreement that the item was appropriate or inappropriate, or in the conclusion that current evidence on the item is insufficient for the item to be incorporated into a definition. A summary of the meeting was sent to the entire panel.

\section{Regional Recurrence}

This section is about regional recurrences. Questions are in the same format as before. Please indicate if you find it appropriate to include the particular events as "regional recurrences" and please let us know if you think any other factors should be taken into account.

Again, some events were listed under local recurrence and/or will be listed under distal recurrence. If this is the case, it is noted in the question.

\section{*11. Recurrence in ipsilateral lymph nodes.}

\section{Is it appropriate to include recurrence in the following locations as a "regional} recurrence"?

The item ipsilateral intramammary lymph node was also listed under "local recurrence" and "second primary breast cancer".

Please note that you will be asked the same question under "distant recurrence".

\begin{tabular}{|c|c|c|c|c|c|c|c|c|c|}
\hline & $\begin{array}{c}\text { 1: Very } \\
\text { inappropriate }\end{array}$ & 2 & 3 & 4 & 5 & 6 & 7 & 8 & $\begin{array}{c}\text { 9: Very } \\
\text { appropriate }\end{array}$ \\
\hline $\begin{array}{l}\text { Ipsilateral axillary lymph } \\
\text { node }\end{array}$ & C & $c$ & C & C & $c$ & $c$ & $c$ & C & C \\
\hline $\begin{array}{l}\text { Ipsilateral infraclavicular } \\
\text { lymph node }\end{array}$ & 0 & 0 & C & 0 & 0 & 0 & 0 & 0 & 0 \\
\hline $\begin{array}{l}\text { Ipsilateral supraclavicular } \\
\text { lymph node }\end{array}$ & c & C & C & c & C & C & C & C & 0 \\
\hline $\begin{array}{l}\text { Ipsilateral internal } \\
\text { mammary / parasternal } \\
\text { lymph node }\end{array}$ & 0 & 0 & c & 0 & 0 & 0 & 0 & 0 & 0 \\
\hline $\begin{array}{l}\text { Ipsilateral intramammary } \\
\text { lymph node }\end{array}$ & c & $c$ & 0 & $r$ & $c$ & $c$ & $c$ & C & $c$ \\
\hline
\end{tabular}

Other important factors or remarks

Figure 2.2 Example of a question from the first questionnaire 


\section{Statistical analysis}

The results were exported to MS Excel 2010 (Microsoft Corporation, Redmond WA). Consensus was present if the panel rated the event appropriate or inappropriate (panel median 1-3 or 7-9) without disagreement, which was tested using the IPRAS (interpercentile range adjusted for symmetry) formula in accordance to the RAND/UCLA Appropriateness Method Manual. For more detailed information on the analysis and the definition of disagreement, see the Supplementary Methods section (Appendix 2.I).

\section{Results}

\section{Panel formation}

Email invitations were sent to 40 persons (10 surgical oncologists, 10 medical oncologists, eight radiation oncologists, five pathologists, three epidemiologists, and four other professionals involved in designing, publishing, or funding of breast cancer research). Of 40 persons, 26 were willing to participate and 11 did not respond. Three persons were unwilling to participate, of whom two felt that their expertise was insufficient (breast cancer currently not main field of interest); one person did not agree with the aim of the project.

\section{Characteristics of panel members}

The characteristics of the panel members are summarized in Table 2.1. All clinical breast cancer disciplines are represented. The panel members are affiliated with a variety of professional and research organizations, including American College of Surgeons Oncology Group, American Society of Breast Surgeons, American Society of Clinical Oncology, American Society for Radiation Oncology, Breast International Group, Cochrane Breast Cancer Review Group, Clinical Oncology Society Australia, European Cancer Organisation, European Organisation for Research and Treatment of Cancer, European Society for Medical Oncology, European Society for Radiotherapy and Oncology, European Registration of Cancer Care, International Breast Cancer Study Group, Medical Oncology Group of Australia, National Surgical Adjuvant Breast and Bowel Project, Royal Australian and New Zealand College of Radiologists, Society of Surgical Oncology, as well as several local and national research groups, guideline committees, and professional boards. The above listed institutions themselves were not involved in this project and do not necessarily approve of the consensus. 


\section{Participation}

The first questionnaire was sent to 26 people and completed by 24 . The second questionnaire was sent to all respondents of the first survey, and was completed by 22 of 24. All 24 panel members were invited to the consensus meeting, which took place at the San Antonio Breast Cancer Symposium in December 2013. Eight panel members attended.

\section{First Questionnaire}

The first questionnaire consisted of 122 items in four categories, namely local event, second primary breast cancer, regional event, and distant event. Some items were listed in multiple categories. For instance, recurrence in skin on ipsilateral thoracic wall appeared in the local, regional, and distant categories. After the first round, consensus existed on 67 of 122 items (54.9\%) and disagreement or uncertainty on 33 of 122 items. Based on additional remarks, four of 122 items were disregarded, and 18 of 122 items were replaced or rephrased for clarification.

\section{Second questionnaire}

The second questionnaire consisted of 84 items, namely items on which consensus did not exist in the first round $(n=33)$, items added based on additional comments $(n=24)$, and items which were replaced or clarified ( $n=27$, replacing 18 items from the first survey). After the second round, consensus existed on 24 of 84 (28.6\%) items, in addition to the 67 items on which consensus was reached in the first round.

Table 2.1 Characteristics of panel members ( $n=24$, participants of first questionnaire)

\begin{tabular}{lc}
\hline Characteristics & $\mathrm{N}$ \\
\hline Discipline & 5 \\
Epidemiology & 8 \\
Medical oncology & 1 \\
Pathology & 5 \\
Radiation oncology & 8 \\
Surgical oncology & 1 \\
Other & \\
Sex & 8 \\
Female & 16 \\
Male & \\
Continent & 2 \\
Australia & 12 \\
Europe & 10 \\
North America & \\
\hline
\end{tabular}




\section{Final meeting}

In the final meeting, items on which consensus did not exist after two rounds of questionnaires were discussed. These items concerned a limited number of issues, namely classification of breast cancer in skin and subcutaneous tissue (27 items in categories local, regional, and distant event), distinction between local events and new primary ipsilateral breast cancers (13 items in local event and second primary breast cancer), contralateral lymph nodes (14 items in regional and distant event), and appropriate diagnostics of distant events (seven items).

In general, panel members preferred the word "event" over "recurrence", as the former is more objective and less suggestive of etiology.

The first topic of debate was whether ipsilateral breast cancer should be subclassified as true recurrence or second primary. Several potential factors, such as distance from original tumor, histologic features, and molecular similarity were listed as items in the categories "local event" and "second primary breast cancer". During the questionnaire rounds, there was disagreement regarding the appropriate classification of events occurring in another quadrant of the breast than the original tumor, events with another morphology/histologic subtype, receptor switch (particularly negative to positive), and distinction based on molecular characteristics such as loss of heterozygosity analysis. Finally, for reasons of simplicity, heterogeneity within tumors, and lack of evidence regarding prognostic significance of this distinction, the panel decided during the meeting that all ipsilateral epithelial breast cancer as well as ductal carcinoma in situ (DCIS) should be considered a local event.

The second topic of debate was isolated recurrence in contralateral lymph nodes (ie, axillary, supraclavicular, infraclavicular, parasternal, or internal mammary), in absence of synchronous malignancy in either breast or synchronous distant metastasis. Initially, a distinction was made between contralateral lymph node events after sentinel lymph node biopsy, axillary lymph node dissection, or axillary radiotherapy, as well as after a previously medially located tumor, and after inflammatory breast cancer. These distinctions were removed because of disagreement. Many panel members felt that contralateral lymph node events are associated with a worse prognosis than ipsilateral lymph node events, but a better prognosis than most distant events. Classifying metastatic contralateral nodes as a separate category was considered. During the meeting, consensus was reached that contralateral lymph node events should be considered distant events. The biology and prognostic and therapeutic consequences of contralateral lymph node events should be subject to future research.

The third topic of debate was resectability. It was suggested that irresectable recurrence should be considered distant. The panel concluded that irresectability is subjective and 
should not be a reason to classify an event as distant, although outcome might be worse in particular cases.

Finally, the panel discussed whether tissue sampling should be mandatory for a first, solitary lesion suspected for metastasis on imaging. The panel recommended biopsy if feasible. If tissue sampling is not possible (which the panel considered to be very rare), unconfirmed first solitary metastasis is acceptable at the discretion of the treating physician or interdisciplinary tumor board. Multiple lesions consistent with metastases on imaging are acceptable with- out tissue sampling, although even in these cases, histologic confirmation should be performed if feasible.

\section{Consensus-based definitions}

The consensus is summarized in Table 2.2. Consensus was reached on 134 items in four categories. All epithelial breast cancer or DCIS in the ipsilateral (former) breast, or in skin and subcutaneous tissue on the ipsilateral thoracic wall, are considered local events. Second primary breast cancer is epithelial breast cancer in the contralateral breast (with or without nodal involvement on that side).

Regional events are breast cancer in ipsilateral lymph nodes (axillary, supra- clavicular, infraclavicular, internal mammary, and intramammary). A distant event is breast cancer anywhere else than listed above. Thus, distant events include breast cancer involving the sternal bone, isolated contralateral lymph nodes (axillary, supraclavicular, infraclavicular, parasternal, and internal mammary) in absence of synchronous ipsilateral or contralateral breast malignancy or distant metastasis, as well as skin and subcutaneous tissue outside the ipsilateral thoracic wall. Pathology confirmation of a first, solitary lesion suspected for metastasis on imaging is highly recommended if feasible. Multiple metastases on imaging are acceptable without tissue sampling.

\section{Discussion}

This project used the RAND/UCLA Appropriateness method to develop consensus-based, standardized definitions of local event, second primary breast cancer, regional event, and distant event for use in breast cancer research. Adoption of these definitions in breast cancer studies will increase transparency and facilitate comparison of results. 
Table 2.2 Summary of the consensus on the definition of local event, second primary breast cancer, regional event, and distant event for classification of recurrence in breast cancer research

\begin{tabular}{|c|c|}
\hline $\begin{array}{l}\text { Local event } \\
\text { (after mastectomy or breast } \\
\text { conserving therapy) }\end{array}$ & $\begin{array}{l}\text { Any epithelial breast cancer or DCIS in ipsilateral breast tissue } \\
\text { Breast cancer in surgical scar } \\
\text { Breast cancer in biopsy tract } \\
\text { Breast cancer in skin and subcutaneous tissue on the (former) ipsilateral } \\
\text { breast and ipsilateral thoracic wall* } \\
\text { Should NOT include: LCIS, phyllodes tumors, any benign breast lesion, any } \\
\text { breast cancer event involving the sternal bone. }\end{array}$ \\
\hline Second primary breast cancer & $\begin{array}{l}\text { Any epithelial breast cancer in the contralateral breast (with or without } \\
\text { lymph node metastases on that side) }\end{array}$ \\
\hline Regional event & $\begin{array}{l}\text { Breast cancer in ipsilateral axillary, infraclavicular, supraclavicular, internal } \\
\text { mammary/parasternal, or intramammary lymph node }\end{array}$ \\
\hline Distant event & $\begin{array}{l}\text { Breast cancer in any organ other than breast, excluding the items listed } \\
\text { under local event, second primary breast cancer, and regional event. } \\
\text { Therefore also including any breast cancer event involving the sternal bone } \\
\text { Therefore also including breast cancer in contralateral lymph nodes (axillary, } \\
\text { infraclavicular, supraclavicular, and internal mammary), in absence of } \\
\text { synchronous ipsilateral or contralateral breast malignancy or distant } \\
\text { metastasis } \\
\text { Tissue sampling } \\
\text { Pathology confirmation (histology or cytology) of a first, solitary lesion } \\
\text { suspected for metastasis is highly recommended if feasible. If tissue } \\
\text { sampling is impossible, unconfirmed metastasis is acceptable at discretion } \\
\text { of the treating physician. } \\
\text { Multiple lesions consistent with metastases on imaging are acceptable } \\
\text { without pathology confirmation }\end{array}$ \\
\hline
\end{tabular}

\footnotetext{
*Ipsilateral thoracic wall: area between contralateral sternal border medially, posterior axillary line laterally, the clavicle superiorly and the (former) inframammary fold inferiorly.

Abbreviations: DCIS ductal carcinoma in situ, LCIS lobular carcinoma in situ.
}

The definitions are designed for classification of events in research; they are not intended to guide individual patient management. For instance, a recurrence invading the chest wall after mastectomy can be treated with curative intent for one patient, considering it to be a "local" problem, whereas for the next patient it can be considered equivalent to "distant disease" as a consequence of age, comorbidity, and/or extent of the disease. Obviously, this is relevant for managing the individual patient. In contrast, registration of research data requires simplicity and consistency. Additionally, techniques for classification must be available throughout the world. A molecular technique may be promising to distinguish second primary breast cancer from true recurrence. However, if it is not universally available, incorporating it in definitions will compromise reliable comparison of results. 
This consensus is based on the opinion of 24 breast cancer experts. Strengths of this approach include selection of panel members in all disciplines involved in breast cancer care and members of most major research groups and a variety of professional societies and boards. Although the number of panel members (particularly, attendance to the final meeting) is an inherent limitation of a consensus project, we consider the panel to be representative.

Results of a formal consensus project can be seen as a systematic evaluation of expert opinions. Expert opinions do not constitute the highest level of evidence, which is a second limitation of this project. If a higher level of evidence can be obtained, this is desirable. In the case of events in endpoints, this would require consistent evidence concerning prognostic and therapeutic relevance of all items. Ideally, a valid composite endpoint consists of elements that are of similar prognostic significance, importance to patients, and incidence, and are influenced by the intervention to a similar degree. ${ }^{7}$ If this is not the case, reporting the incidence of a composite endpoint may be misleading and differences in prognosis or treatment effect in study arms may not be adequately reflected. Therefore, it would have been appropriate to provide information regarding these criteria for each item. However, in the light of major changes in local treatment, systemic treatment, and diagnostics in the last decades, specific information was not available for most items. The lack of evidence concerning these criteria is both a limitation of this study and the reason why formal expert consensus is a suitable approach. Future research may illuminate prognostic and therapeutic relevance of specific items, prompting adaption of the definitions. In the meantime, however, the problem of inconsistent event definitions is so pressing that the use of standardized definitions is desirable, even if an expert consensus (with its inherent initial disagreement on some topics, as a consensus, by definition, does not reflect everybody's initial opinion) is the highest level of evidence that can be obtained at this moment.

Using uniform definitions of events in breast cancer research is essential for transparency and reliable comparison of results. Earlier, Hudis ${ }^{6}$ and Fumagalli ${ }^{5}$ proposed standardized definitions of endpoints for the neoadjuvant and adjuvant setting. An additional proposal may be expected from the Definition for the Assessment of Time-toevent Endpoints in CANcer trials group. ${ }^{12}$ The current project strengthens these proposals, because uniform definition of endpoints requires uniform definition of included events. The Standardized Definitions for Efficacy End Points in Adjuvant Breast Cancer Trials (STEEP) project by Hudis et al. ${ }^{6}$, for instance, was specifically designed for the adjuvant setting. Although it is specific about inclusion and exclusion of noninvasive lesions in specific endpoints and distinguishes between invasive ipsilateral breast tumor recurrence and local regional recurrence, the STEEP project left room for interpretation 
concerning which events should be considered local, regional, and distant. The current project fills this gap. Therefore, it improves applicability in research on local and regional treatment. It also facilitates presenting incidence of specific events in addition to the primary endpoint, as was suggested by Hudis et al. Adoption of these standardized event definitions will improve transparency and will facilitate comparison of study results. This effect will be particularly pronounced when authors report the incidence of separate events (e.g., number of local events, regional events) in addition to the primary endpoint. In that case, data will always be comparable, even if the primary endpoint differs.

These consensus-based definitions should be adopted in all breast cancer research using clinical outcomes. This includes research collaborative groups, national cancer institutes, and regulatory authorities. They should be integrated in coding rules for data management. They should also be used as building blocks for composite endpoints in publications. In addition, authors should report the incidence of separate events in addition to the incidence of the primary endpoint.

In conclusion, these consensus-based definitions of local event, second primary breast cancer, regional event, and distant event can serve as building blocks for endpoints in breast cancer research. They should be adopted by data managers of breast cancer studies, as well as researchers initiating, conducting, or publishing results of breast cancer research. 


\section{References}

1. Mathoulin-Pelissier S, Gourgou-Bourgade S, Bonnetain F, Kramar A. Survival End Point Reporting in Randomized Cancer Clinical Trials: A Review of Major Journals. J Clin Oncol. 2008;26(22):3721-3726.

2. Meropol NJ. Comparative Effectiveness Research to Inform Medical Decisions: The Need for Common Language. J Clin Oncol. 2012;30(34):4192-4193.

3. Cuzick J. Primary endpoints for randomised trials of cancer therapy. Lancet. 2008;371(9631):2156-2158.

4. Nout RA, Fiets WE, Struikmans $H$, et al. The in- or exclusion of non- breast cancer related death and contralateral breast cancer significantly affects estimated outcome probability in early breast cancer. Breast Cancer Res Treat. 2008;109(3):567-572.

5. Fumagalli D, Bedard PL, Nahleh Z, et al. A common language in neoad- juvant breast cancer clinical trials: proposals for standard definitions and endpoints. Lancet Oncol. 2012;13(6):e240-e248.

6. Hudis CA, Barlow WE, Costantino JP, et al. Proposal for Standardized Definitions for Efficacy End Points in Adjuvant Breast Cancer Trials: The STEEP System. J Clin Oncol. 2007;25(15):2127-2132.

7. Montori VM, Permanyer-Miralda G, Ferreira-Gonzalez I, et al. Validity of composite end points in clinical trials. BMJ. 2005;330(7491):594-596.

8. Fitch K, Bernstein SJ, Aguilar MS, et al. The RAND/UCLA Appropriateness Method User's Manual. Santa Monica, CA: RAND; 2001.

9. Fink A, Kosecoff J, Chassin M, Brook RH. Consensus methods: character- istics and guidelines for use. Am J Public Health. 1984;74(9):979-983.

10. Jones J, Hunter D. Consensus methods for medical and health services research. BMJ. 1995;311(7001): 376-380.

11. Linstone HA, Turoff M. The Delphi method: techniques and applications: Addison-Wesley Pub. Co., Advanced Book Program; 1975.

12. Bellera CA, Pulido M, Gourgou S, et al. Protocol of the Definition for the Assessment of Time-to-event Endpoints in CANcer trials (DATECAN) project: Formal consensus method for the development of guidelines for standardised time-to-event endpoints' definitions in cancer clinical trials. Eur J Cancer. 2013;49(4):769-781. 


\section{Appendix 2.1}

\section{Supplementary Methods Section: analysis of questionnaire results}

Panel members score each item on a nine-point scale, where 1 equals "Very inappropriate" and 9 equals "Very appropriate". Analysis of rating of events was conducted using MS Excel 2010. The formulas that were used are listed in Table A.

Table A MS Excel 2010 formulas used to assess appropriateness and disagreement

\begin{tabular}{ll}
\hline Median of panel rating & $=\operatorname{MEDIAN}(\mathrm{x}: \mathrm{x})$ \\
$30^{\text {th }}$ percentile & $=$ PERCENTILE.EXC $(\mathrm{x}: \mathrm{x} ; 0,3)$ \\
$70^{\text {th }}$ percentile & $=$ PERCENTILE.EXC $(\mathrm{x}: \mathrm{x} ; 0,7)$ \\
Interpercentile range $30^{\text {th }}-70^{\text {th }}$ & $=\left[70^{\text {th }}\right.$ percentile $]-\left[30^{\text {th }}\right.$ percentile $]$ \\
Central point IPR & $=\left(\left[70^{\text {th }}\right.\right.$ percentile $]+\left[30^{\text {th }}\right.$ percentile $\left.]\right) / 2$ \\
Asymmetry Index & $=A B S(5-[$ central point IPR $])$ \\
IPRAS & $=2,35+\left(1,5^{*}[\right.$ Asymmetry index $\left.]\right)$ \\
IPRAS-IPR & $=[$ IPRAS $]-[$ IPR $]$ \\
\hline
\end{tabular}

Consensus was defined as a panel median between 1 and 3 (Inappropriate) or between 7 and 9 (Appropriate) without disagreement. Inversely, this means that consensus did not exist if the panel median was between 4 and 6 (Uncertain), or if the answers varied so much that the definition of disagreement was met.

Disagreement was assessed according to the IPRAS Method as described in the RAND/UCLA Appropriateness Method Manual ${ }^{8}$. Traditionally, the Appropriateness Method defined disagreement based on the amount of panel members that voted outside the 3-point range that contained the median. However, for panels consisting of more than nine members, another method, based on the InterPercentile Range (IPR), is recommended. A smaller IPR of the panel's answers reflects more agreement. The 30th70th percentile range is used because it most accurately reflects the traditional RAND/UCLA definition of disagreement. However, the IPR in itself is not sufficient to assess agreement. One also needs to adjust for symmetry of the answers, because the IPR in itself does not take into account if the answers are at the same side of the rating scale of if there are extreme differences between panel members (reflected in answers distributed symmetrically on both sides of the rating scale). To illustrate the importance of correcting for symmetry, an example for a nine member panel is shown in table $\mathrm{B}$. The IPR is the same for both samples, although it is clear that panel members did not agree as much on question 1 as they did on question 2. To correct for this problem, RAND/UCLA developed a formula called IPRAS (InterPercentile Range Adjusted for 
Symmetry). In short, the IPRAS method determines if disagreement is present, based on the IPR of the ratings of the panel members, adjusted for symmetry.

Table B Example of the difference between interpercentile range (IPR) and interpercentile range adjusted for symmetry (IPRAS) in a nine member panel

\begin{tabular}{|c|c|c|c|c|c|c|c|c|c|c|c|}
\hline & \multicolumn{9}{|c|}{ Panel ratings } & \multirow[t]{2}{*}{ IPR 30\%-70\% } & \multirow[t]{2}{*}{ IPRAS-IPR } \\
\hline & $a$ & $b$ & c & $d$ & $e$ & $f$ & $g$ & $h$ & $i$ & & \\
\hline Item 1 & 1 & 1 & 3 & 5 & 5 & 5 & 7 & 9 & 9 & 4 & $-1,65$ : disagreement \\
\hline Item 2 & 1 & 1 & 1 & 3 & 3 & 3 & 5 & 5 & 5 & 4 & 1,35: agreement \\
\hline
\end{tabular}

The IPRAS formula (see Table A) contains fixed variables and a measure of asymmetry, the Asymmetry Index. As the answers of the panel (and therefore the symmetry of the answers) differ per item, every item has its own Asymmetry Index. The IPRAS reflects the broadest IPR that would constitute agreement at a certain Asymmetry Index. Next, the IPRAS can be compared to the actual IPR of the ratings of the panel. If the actual IPR is larger than the calculated IPRAS, this means disagreement is present taking into account the asymmetry of the answers. Therefore, IPRAS-IPR is $<0$ if the actual IPR of the ratings is larger than the range that would be the threshold for disagreement at the particular level of asymmetry of the answers. Thus, IPRAS-IPR indicates agreement if $>0$ and disagreement if $<0$.

In the example in Table B, scores on both items have a 30\%-70\% IPR of 4 . For item 1, the calculated IPRAS minus the observed IPR results in disagreement, reflecting the fact that in question 1, panel members answered on both extremes of the scale, whereas in question 2, there was some uncertainty but answers were generally on the low side of the rating scale, which was recognized as in this case IPRAS-IPR does indicate agreement. 


\section{Chapter 3}

Contralateral lymph node recurrence in breast cancer: regional event rather than distant metastatic disease. $\mathrm{A}$ systematic review of the literature Moossdorff M, Vugts G, Maaskant-Braat AJG, Strobbe LJA, Voogd AC, Nieuwenhuizen GAP, Smidt ML

* MM and GV are co-first authors on this chapter Eur J Surg Oncol 2015; 41: 1128-1136 


\section{Abstract}

\section{Aims}

After treatment for breast cancer, some patients experience a contralateral lymph node recurrence (CLNR). Traditionally, contralateral nodes are considered a distant site. However, aberrant lymph drainage after previous surgery is common. This might indicate that CLNR is a regional event. This study aimed to review the literature to determine prognosis after CLNR.

\section{Methods}

PubMed was searched up until July 2014. Articles on CLNR with or without ipsilateral breast tumour recurrence (IBTR), and repeat sentinel node (SN) studies reporting on positive contralateral nodes were included. Exclusion criteria were synchronous contralateral breast cancer and synchronous distant events.

\section{Results}

24 articles were included, describing 48 patients. Of these 48, 26 patients had an isolated CLNR, 7 IBTR and clinically detected CLNR and 15 IBTR with a positive contralateral repeat SN. Isolated CLNR occurred earlier (45.9 months) than IBTR with CLNR (126.6 months, $p<0.001)$ or with a positive contralateral repeat SN (217.2, $p=0.02)$. Surgical treatment was described for 38 patients, and consisted of axillary lymph node dissection for 34 (89.5\%). Information on adjuvant therapy was available for 27 patients, 21 (77.8\%) received chemotherapy. Follow-up information after CLNR was available for 23 patients (47.9\%). Mean follow-up was 50.3 months. Overall survival and disease-free survival were $82.6 \%$ [95\% $\mathrm{Cl} 67.1-98.1]$ and $65.2 \%$ [45.7-84.7] respectively at last followup.

\section{Conclusions}

Although observed in a small population, the survival of CLNR is not comparable to distant disease. Most patients received locoregional and systemic treatment suggesting a curative approach. This indicates that CLNR should be regarded as a regional event. 


\section{Introduction}

After curative treatment for breast cancer, a small proportion of patients experience a contralateral lymph node recurrence during follow-up. When affected at initial diagnosis, contralateral lymph nodes (CLNs) are traditionally considered to be a result of systemic dissemination. ${ }^{1}$

However, lymphoscintigraphy studies in patients who previously underwent surgery of the breast or axilla frequently show lymph drainage to contralateral nodal basins, such as the contralateral axilla, internal mammary chain or periclavicular sites. ${ }^{2-9}$ Hypothetically, these aberrant drainage patterns might indicate that a contralateral lymph node recurrence (CLNR) after previous treatment for breast cancer should be considered as a regional event, rather than systemic disease.

The prognostic impact and therapeutic consequences of CLNRs are not clear. If prognosis of CLNR is comparable to the prognosis of an ipsilateral lymph node recurrence it would support treatment as a regional event, aiming for regional control with curative rather than palliative intent. Prognosis of a CLNR may depend on tumour and treatment related factors. First, prognosis may be affected by synchronous events; CLNR can occur isolated (i.e. without malignancy in either breast or other distant events), or synchronous to an ipsilateral breast tumour recurrence (IBTR), or distant event. In metastatic breast cancer, prognosis is determined mainly by the distant event. In patients with a CLNR without distant metastases, prognosis and the influence of concurrent IBTR are unclear. Another relevant prognostic factor may be the detection method of CLNR. CLNR can be clinically evident with palpable nodes at physical examination and confirmed by cytological or histological examination. CLNR could also be detected as part of the diagnostic workup for an IBTR. Furthermore, the introduction of repeat sentinel node biopsy (SNB) in patients with an IBTR may lead to the detection of tumour positive contralateral sentinel nodes, also to be considered as CLNR. ${ }^{2,5,6,8,10-12}$ These lymph node metastases would have previously gone unnoticed, and may have a different prognostic impact compared to clinically manifest CLNRs. Initial locoregional treatment defines the chance of developing contralateral lymph drainage patterns, as is shown in repeat SNB studies. Patients who previously underwent axillary lymph node dissection (ALND) more often develop contralateral lymph drainage. ${ }^{12}$

In this systematic review of the available literature, we have identified and described all patients with CLNR after previous curative treatment for breast cancer, with or without synchronous IBTR without metastases to other distant sites. We aim to evaluate the prognosis of CLNR. 


\section{Methods}

\section{Search}

The PubMed database (including MEDLINE) was searched until July 2014 using the following terms as free terms and Mesh terms: breast neoplasms, breast cancer, lymph nodes, contralateral, axilla. The full strategy is presented in Appendix 3.I.

\section{Selection}

The selection process of the articles for this review is summarized in Figure 3.1. The abstracts that were retrieved by the search were screened independently by two authors (GV and MM) for eligibility, based on predefined inclusion and exclusion criteria. Articles were eligible if they described breast cancer patients, described recurrence in CLNs, or studied repeat SNB in recurrent breast cancer. Editorials, conference reports, comments on other studies, and animal studies were excluded. Articles were excluded if they described patients with synchronous contralateral breast cancer (i.e. on the same side as the CLNR), synchronous distant events, synchronous CLN involvement at initial diagnosis (i.e. the contralateral lymph node was no recurrence), patients whose CLN was not breast cancer (i.e. benign, non-breast malignancies), and if authors made no distinction between CLNR and other distant events. Patients with isolated tumour cells (ITC) in a contralateral sentinel node were considered node negative and therefore not taken into account for this analysis. Patients with micrometastases in contralateral repeat sentinel nodes were considered node positive, and described separately. Of the selected articles, the full text as well as the reference list were reviewed independently by two authors (GV and MM). If the reference list contained possible eligible articles, these were included. Disagreement was solved by discussion. From publications reporting on multiple individual patients, only those individuals meeting the inclusion criteria were selected for this review.

\section{Data-extraction and statistical analysis}

Data extraction was performed independently by two authors (GV and MM). Disagreement was solved by discussion. The following characteristics were extracted from included publications: study design, whether it concerned CLNR with IBTR or isolated CLNR, initial TNM-classification, initial treatment (axillary, breast, systemic), time from primary breast cancer to CLNR, detection method, number and location of affected CLNs, presence of synchronous metastatic ipsilateral lymph nodes, the method of excluding occult breast cancer on the side of the CLNR, the method of excluding synchronous distant metastasis, treatment of the CLNR (axillary, breast, systemic), and 
outcome (disease free survival, overall survival and months of follow-up). The available data were collected; means and medians were calculated for the period of time from the occurrence of a primary breast tumour to CLNR. These data were stratified for isolated CLNR, IBTR and synchronous CLNR and IBTR with a tumour positive contralateral sentinel node. Time to CLNR within these groups of patients was compared using the Mann Whitney $U$ Test. P-values $<0.05$ were considered statistically significant. For overall and disease- free survival during follow-up after CLNR, 95\% confidence intervals (CI) were calculated.

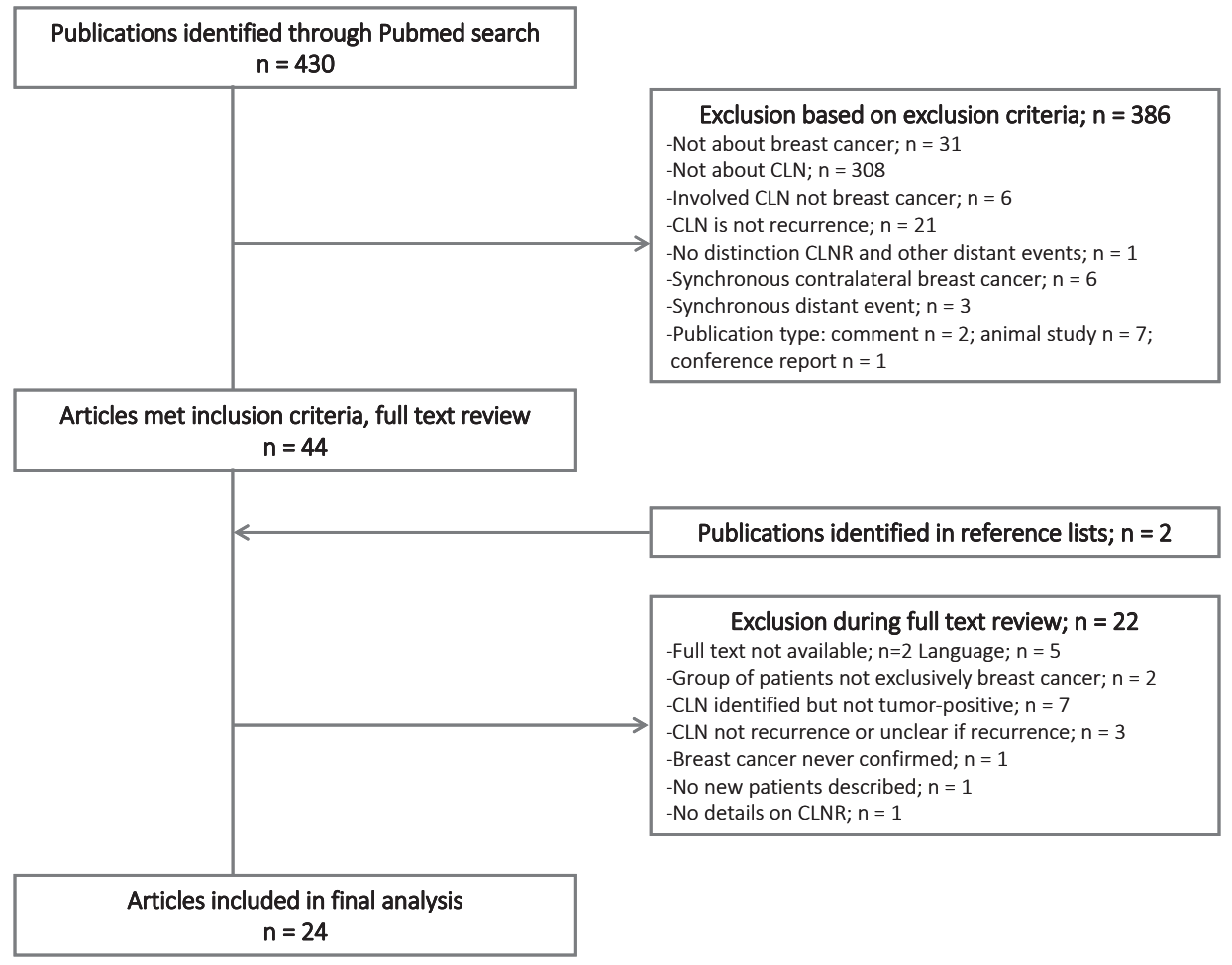

Figure $3.1 \quad$ Flow-chart of search strategy

CLN: contralateral lymph node, CLNR: contralateral lymph node recurrence

\section{Results}

\section{Selection of publications}

The selection process is summarized in Figure 3.1. The PubMed search strategy yielded 430 abstracts. Of these, 386 publications were excluded based on the exclusion criteria. 
The remaining 44 articles were subjected to full text review. Through a manual search of the reference lists, 2 additional eligible articles were obtained. In this stage, 22 publications were excluded. Finally, 24 articles were included in the final analysis. $^{6,8,9,11-31}$

\section{Characteristics of the included studies}

The characteristics of the included studies are presented in Table 3.1. Of the 24 included articles, 15 were studies and case reports describing patients with CLNR with or without a synchronous IBTR, 9 were studies and case reports describing IBTR with a contralateral positive sentinel node. All manuscripts were published between 1995 and 2014. In the 24 selected studies, a total of 48 eligible patients were described, ranging from 1 to 6 per publication.

Table 3.1 Articles included for final analysis

\begin{tabular}{|c|c|c|c|c|c|}
\hline \multirow[t]{2}{*}{ Author } & \multirow[t]{2}{*}{ Year } & \multirow[t]{2}{*}{$\mathrm{N}$} & \multirow[t]{2}{*}{ Article type } & \multicolumn{2}{|c|}{ Detection method } \\
\hline & & & & Clinical & Repeat SNB \\
\hline Jaffer & 1995 & 1 & Case report & $x$ & \\
\hline Daoud & 1998 & 3 & Retrospective case series & $x$ & \\
\hline Lim & 2004 & 1 & Case report & & $x$ \\
\hline Schlechter & 2004 & 1 & Retrospective case series & $x$ & \\
\hline Agarwal & 2005 & 1 & Prospective repeat SNB study & & $x$ \\
\hline Roumen & 2006 & 2 & Prospective repeat SNB study & & $x$ \\
\hline Taback & 2006 & 2 & Prospective repeat SNB study & & $x$ \\
\hline Huston & 2007 & 6 & Retrospective case series & $x$ & \\
\hline Wellner & 2007 & 1 & Case report & $x$ & \\
\hline Koizumi & 2008 & 1 & Retrospective case series & & $x$ \\
\hline Kroon & 2008 & 1 & Case report & $x$ & \\
\hline Lanitis & 2009 & 2 & Retrospective case series & $x$ & \\
\hline Tasevki & 2009 & 1 & Case report & & $x$ \\
\hline Van der Ploeg & 2009 & 2 & Retrospective analysis of prospective cohort & $x$ & \\
\hline Kinoshita & 2010 & 1 & Case report & $x$ & \\
\hline Kim & 2011 & 2 & Retrospective case series & $x$ & \\
\hline Morcos & 2011 & 6 & Retrospective case series & $x$ & \\
\hline Herold & 2011 & 1 & Case report & $x$ & \\
\hline Sabate & 2011 & 1 & Case report & $x$ & \\
\hline Maaskant-Braat & 2013 & 5 & Prospective repeat SNB study & & $x$ \\
\hline Kiluk & 2014 & 3 & Retrospective case series & $x$ & \\
\hline Nishimura & 2014 & 2 & Case report & & $x$ \\
\hline Pasta & 2014 & 1 & Case report & $x$ & \\
\hline Tokmak & 2014 & 1 & Prospective repeat SNB study & & $x$ \\
\hline Total & & 48 & & & \\
\hline
\end{tabular}

N Number of patients; SNB sentinel node biopsy 


\section{Characteristics of patients with CLNR}

All patients included in this systematic review had a history of breast cancer. Tumour characteristics and treatment of these initial breast cancers are shown in Table 3.2.

Table 3.2 Characteristics of the primary breast tumor and treatment in patients with CLNR.

\begin{tabular}{|c|c|c|c|c|c|}
\hline Characteristics & & $\begin{array}{l}\text { CLNR without } \\
\text { IBTR }\end{array}$ & $\begin{array}{c}\text { IBTR with CLNR } \\
\text { (clinically detected) }\end{array}$ & $\begin{array}{l}\text { IBTR with CLNR } \\
\text { (SNB detected) }\end{array}$ & Total \\
\hline Total & & 26 & 7 & 15 & 48 \\
\hline \multirow[t]{5}{*}{ T-stage } & Tis & 0 & 0 & 1 & $1(2.1 \%)$ \\
\hline & $\mathrm{T} 1$ & 5 & 3 & 0 & $8(16.7 \%)$ \\
\hline & $\mathrm{T} 2$ & 5 & 2 & 1 & $8(16.7 \%)$ \\
\hline & $\mathrm{T} 4$ & 1 & 0 & 0 & $1(2.1 \%)$ \\
\hline & Unknown & 15 & 2 & 13 & $30(62.5 \%)$ \\
\hline \multirow[t]{6}{*}{$\mathrm{N}$-stage } & NO & 8 & 3 & 1 & $12(25.0 \%)$ \\
\hline & N1mi & 0 & 1 & 0 & $1(2.1 \%)$ \\
\hline & N1 & 1 & 1 & 0 & $2(4.2 \%)$ \\
\hline & $\mathrm{N} 2$ & 2 & 0 & 0 & $2(4.2 \%)$ \\
\hline & N3 & 1 & 0 & 0 & $1(2.1 \%)$ \\
\hline & Unknown & 14 & 2 & 14 & $30(62.5 \%)$ \\
\hline \multirow[t]{3}{*}{ ER receptor status } & Positive & 6 & 2 & 0 & $8(16.7 \%)$ \\
\hline & Negative & 6 & 2 & 0 & $8(16.7 \%)$ \\
\hline & Unknown & 14 & 3 & 15 & $32(66.7 \%)$ \\
\hline \multirow[t]{3}{*}{ PR receptor status } & Positive & 4 & 2 & 0 & $6(12.5 \%)$ \\
\hline & Negative & 8 & 2 & 0 & $10(20.8 \%)$ \\
\hline & Unknown & 14 & 3 & 15 & $32(66.7 \%)$ \\
\hline \multirow[t]{3}{*}{ HER2 receptor status } & Positive & 5 & 0 & 0 & $5(10.4 \%)$ \\
\hline & Negative & 4 & 2 & 0 & $6(12.5 \%)$ \\
\hline & Unknown & 17 & 5 & 15 & 37 (77.1\%) \\
\hline \multirow[t]{3}{*}{ Breast treatment } & $\mathrm{BCT}$ & 10 & 5 & 11 & $26(54.2 \%)$ \\
\hline & Mastectomy & 4 & 0 & 1 & $5(10.4 \%)$ \\
\hline & Unknown & 12 & 2 & 3 & $17(35.4 \%)$ \\
\hline \multirow[t]{4}{*}{ Axillary treatment } & ALND & 11 & 7 & 11 & $29(60.42 \%)$ \\
\hline & SNB & 2 & 0 & 1 & $3(6.25 \%)$ \\
\hline & None & 0 & 0 & 1 & $1(2.1 \%)$ \\
\hline & Unknown & 13 & 0 & 2 & $15(31.25 \%)$ \\
\hline \multirow[t]{3}{*}{ Chemotherapy } & Yes & 6 & 4 & 2 & $12(25.0 \%)$ \\
\hline & No & 4 & 1 & 2 & $7(14.6 \%)$ \\
\hline & Unknown & 16 & 2 & 11 & $29(60.4 \%)$ \\
\hline \multirow[t]{3}{*}{ Endocrine therapy } & Yes & 3 & 1 & 1 & 5 (10.4\%) \\
\hline & No & 6 & 3 & 1 & $10(20.8 \%)$ \\
\hline & Unknown & 17 & 3 & 13 & $33(68.75 \%)$ \\
\hline \multirow[t]{3}{*}{ Trastuzumab } & Yes & 1 & 0 & 0 & $1(2.1 \%)$ \\
\hline & No & 6 & 4 & 2 & $12(25.0 \%)$ \\
\hline & Unknown & 19 & 3 & 13 & 35 (72.9\%) \\
\hline
\end{tabular}

CLNR contralateral lymph node recurrence; IBTR ipsilateral breast tumor recurrence; SNB sentinel node biopsy; $T$-stage tumor stage; $N$-stage nodal stage; $B C T$ breast conserving therapy; $A L N D$ axillary lymph node dissection; $E R$ estrogen; $P R$ progesterone 
None of the included patients presented with inflammatory breast cancer. Twenty-six patients (54.2\%) had undergone breast conserving therapy (BCT) and 5 (10.4\%) mastectomy, of whom 2 (4.2\%) also underwent chest wall irradiation. The initial breast treatment was not specified in the publication for 17 (35.4\%) patients. Previous surgery of the axilla consisted of axillary lymph node dissection (ALND) in 29 (60.4\%) patients, $3(6.3 \%)$ patients underwent SNB only, 1 (2.1\%) patient did not receive any axillary treatment, and axillary treatment was not specified for 15 (31.3\%) patients. For 19 patients, the use of adjuvant chemotherapy for the primary tumour was registered; chemotherapy was administered in 12 (63.2\%) (Table 3.2). Administration of endocrine therapy was described for 15 of the 48 patients (31.25\%), 5 of whom received some form of endocrine therapy. In patients with an isolated CLNR, the mean time interval from primary tumour to CLNR was 45.9 months. This was 126.6 months in patients with CLNR and synchronous IBTR and 217.2 months in IBTR patients with a positive contralateral sentinel node. Time from primary tumour to the detection of CLNR was shorter in patients with an isolated CLNR; this difference was statistically significant compared to patients with IBTR and clinically detected CLNR $(p<0.001)$, as well as compared to patients with IBTR and a positive contralateral sentinel node $(p=0.02$; Table 3.3).

\section{Detection method}

The 48 eligible patients were divided into 3 groups, based on the type of their CLNR. The first group ( $N=26)$ concerned patients with an isolated CLNR; the second group $(N=7)$ consists of patients with an IBTR and synchronous CLNR detected clinically (i.e. at physical examination or during the diagnostic work-up); the third group ( $N=15)$ consists of patients with an IBTR and a positive contralateral sentinel node (subclinical disease). Physical examination was the most common method (45.5\% of patients) to detect clinical CLNR (Table 3.3). The contralateral axilla was the most common basin for a CLNR, with $97.9 \%$ of all CLNRs. One patient with a CLNR in the internal mammary chain was described. ${ }^{9}$ In a total of 19 patients (39.6\%) the method of excluding a contralateral breast tumour was recorded. This varied between prophylactic contralateral mastectomy $(\mathrm{N}=2)$, to several radiological examinations; breast imaging was not specified in one patient, for other patients mammography only $(\mathrm{N}=5)$, mammography and $\mathrm{MRI}(\mathrm{N}=3), \mathrm{MRI}$ only $(\mathrm{N}=3), \mathrm{MRI}$ and PET-CT $(\mathrm{N}=3)$ or PET-CT only $(\mathrm{N}=2)$ were performed. 


\section{Regional and systemic treatment}

Almost all patients underwent surgery for their CLNR. ALND was performed in 34 (70.8\%) of all patients, in 3 of which $(6.3 \%)$ it was combined with regional radiotherapy. In the remaining patients, axillary radiotherapy only $(\mathrm{N}=2)$, resection of the affected node $(\mathrm{N}=1)$ or no axillary treatment at all $(\mathrm{N}=1)$ was carried out. In 10 patients (20.8\%) regional treatment was not described (Table 3.3). Chemotherapy following CLNR was administered in 21 patients (43.8\%), endocrine therapy in 7 patients (14.6\%). In $43.8 \%$ of patients, administration of adjuvant systemic treatment was not described. Of the 22 patients with a synchronous IBTR, 21 underwent mastectomy and 1 patient underwent BCT.

Table 3.3 Detection and treatment of CLNR

\begin{tabular}{|c|c|c|c|c|}
\hline & CLNR without IBTR & $\begin{array}{c}\text { IBTR with CLNR } \\
\text { (clinically detected) }\end{array}$ & $\begin{array}{l}\text { IBTR with CLNR } \\
\text { (SNB detected) }\end{array}$ & Total \\
\hline $\mathrm{N}$ & 26 & 7 & 15 & 48 \\
\hline \multicolumn{5}{|l|}{ Months to recurrence } \\
\hline Mean & 45.9 & 126.6 & 217.2 & 127.8 \\
\hline Median & 34 & 108 & 138 & 69.5 \\
\hline \multicolumn{5}{|l|}{ Detection method } \\
\hline Clinically & $12(46.2 \%)$ & $3(42.9 \%)$ & 0 & $15(31.3 \%)$ \\
\hline US & $2(7.7 \%)$ & 0 & 0 & $2(4.2 \%)$ \\
\hline PET & & $2(28.5 \%)$ & 0 & $2(4.2 \%)$ \\
\hline Repeat SNB ${ }^{a}$ & $1(3.8 \%)$ & 0 & $15(100 \%)$ & $16(33.3 \%)$ \\
\hline Unknown & $11(42.3 \%)$ & $2(28.6 \%)$ & 0 & $13(27.1 \%)$ \\
\hline \multicolumn{5}{|l|}{ LN location } \\
\hline Axilla & $26(100 \%)$ & $6(85.7 \%)$ & 15 (100\%) & 47 (97.9\%) \\
\hline Internal mammary & 0 & $1(14.3 \%)$ & 0 & $1(2.1 \%)$ \\
\hline \multicolumn{5}{|l|}{ LN treatment } \\
\hline ALND & $17(65.4 \%)$ & $5(71.4 \%)$ & $9(60 \%)$ & $31(64.6 \%)$ \\
\hline ALND \& RTX & $1(3.8 \%)$ & $1(14.3 \%)$ & $1(6.7 \%)$ & $3(6.3 \%)$ \\
\hline $\mathrm{RTx}$ & $1(3.8 \%)$ & 0 & $1(6.7 \%)$ & $2(4.2 \%)$ \\
\hline Resection $^{\mathrm{b}}$ & $1(3.8 \%)$ & 0 & 0 & $1(2.1 \%)$ \\
\hline None & $1(3.8 \%)$ & 0 & 0 & $1(2.1 \%)$ \\
\hline Unknown & $5(19.2 \%)$ & $1(14.3 \%)$ & $4(26.7 \%)$ & $10(20.8 \%)$ \\
\hline \multicolumn{5}{|l|}{ Systemic treatment } \\
\hline Chemotherapy & $7(26.9 \%)$ & $4(57.1 \%)$ & $7(46.7 \%)$ & $18(37.5 \%)$ \\
\hline Chemo- \& endocrine therapy & $3(11.5 \%)$ & 0 & 0 & $3(6.3 \%)$ \\
\hline Endocrine therapy & 0 & 0 & $4(26.7 \%)$ & $4(8.3 \%)$ \\
\hline None & $2(7.7 \%)$ & 0 & $1(6.7 \%)$ & $3(6.3 \%)$ \\
\hline Unknown & $14(53.8 \%)$ & $3(42.9 \%)$ & $4(26.7 \%)$ & $21(43.8 \%)$ \\
\hline
\end{tabular}

a a prophylactic contralateral mastectomy and SNB was carried out; ${ }^{b}$ resection of the affected lymph nodes only, no completion ALND 


\section{Follow-up after CLNR}

To assess prognosis after CLNR, follow-up data were analyzed. Follow-up data were available for 23 patients (47.9\%). Mean available follow-up time for all patients was 50.3 months. Overall survival was $82.6 \%(95 \% \mathrm{Cl} 67.1-98.1)$ and disease-free survival was $65.2 \%(95 \% \mathrm{Cl} 45.7-84.7)$. In patients with an isolated CLNR ( $\mathrm{N}=13)$ the mean available follow-up time was 69.2 months (range: 7-408) while this was 23.5 months (range: 1236) in patients with CLNR and an IBTR $(\mathrm{N}=4)$ and 27 months (range: 6-72) in IBTR patients with a positive contralateral sentinel node $(\mathrm{N}=6)$. Of the patients with isolated CLNR, 76.9\% (95\% Cl 54-99.8\%) was alive after the mean follow-up time of 69.2 months (Table 3.4). Disease-free survival was lower: $46.1 \%$ of patients with isolated CLNR (95\% $\mathrm{Cl}$ 19-73.2) were alive without locoregional recurrence or metastases at last follow-up. Disease free survival of patients with IBTR and synchronous CLNR was $100 \%(\mathrm{~N}=4)$. Overall survival of patients with IBTR and a positive contralateral sentinel node was 83.4\% (95\% Cl 53.5-100), with all surviving patients being disease-free at last follow-up.

Table $3.4 \quad$ Follow-up and survival after CLNR

\begin{tabular}{lcccc}
\hline & CLNR without IBTR & $\begin{array}{c}\text { IBTR with CLNR } \\
\text { (clinically detected) }\end{array}$ & $\begin{array}{c}\text { IBTR with CLNR (SNB } \\
\text { detected) }\end{array}$ & Total \\
\hline $\mathrm{N}$ & 26 & 7 & 15 & 48 \\
Follow-up data available & $13(50 \%)$ & $4(57.1 \%)$ & $6(40 \%)$ & $23(47.9 \%)$ \\
Mean follow-up after & 69.2 & 23.5 & 27 & 50.3 \\
$\begin{array}{l}\text { CLNR (months) } \\
\text { Survival at last follow-up }\end{array}$ & 10 & 4 & 5 & 19 \\
$\quad \begin{array}{l}\text { Percentage } \\
\text { 95\% Cl }\end{array}$ & $76.9 \%$ & $100 \%$ & $83.3 \%$ & $82.6 \%$ \\
$\begin{array}{l}\text { Disease free at last } \\
\text { follow-up }\end{array}$ & $54-99.8$ & $43.5-100$ & $67.1-98.1$ \\
$\quad \begin{array}{l}\text { Percentage } \\
\text { 95\% Cl }\end{array}$ & 6 & $46.1 \%$ & $53 \%$ & 15 \\
\hline
\end{tabular}

CLNR contralateral lymph node recurrence; IBTR ipsilateral breast tumor recurrence; SNB sentinel node biopsy; $N$ number of patients; $\mathrm{Cl}$ Confidence Interval.

\section{Discussion}

Currently, the prognostic impact of CLNR is unclear. This study systematically reviewed literature on the detection, treatment and prognostic impact of CLNRs. Literature is scarce and consists of mostly small studies and case re- ports, in which the level of detail and completeness of the reported data varied. However, in this series of 48 patients with CLNR (of whom 23 with available follow-up data) we observed that the prognosis of CLNR (overall survival of $82.6 \%$ after a mean of 50.3 months) is not comparable to the 
prognosis of metastatic breast cancer. Furthermore, the majority of the patients received surgical (92.1\% of patients) and systemic treatment (88.9\%), suggesting a curative instead of palliative intent. Therefore, the classification of CLNR as distant disease does not seem justified.

The origin of CLNR may be different to the origin of metastatic disease. Distant metastases occur due to systemic circulating spread of tumour cells. CLNR might originate due to aberrant lymph drainage from the ipsilateral breast to contralateral nodal basins, similar to ipsilateral lymph node recurrences. Lymphatic drainage from the breast towards the ipsilateral axilla is well established and was described for the first time by the French anatomist Sappey, in $1874 .^{32}$ Lymph drainage outside the ipsilateral axilla occurs in $20-57 \%$ of primary breast cancer patients. ${ }^{3,5,33}$ This depends on the sentinel node identification technique (e.g. injection site, amount and type of tracer), and consists mainly of drainage to the internal mammary chain. Drainage to the contralateral axilla is more rare, occurring in 0-2\% at initial diagnosis. ${ }^{34,35}$ However, after previous surgery or radiotherapy of the breast or axilla, aberrant drainage patterns are more common. Overall, drainage outside of the ipsilateral axilla is described in $18-70 \%$ after previous surgery or radiotherapy for breast cancer. ${ }^{4-6}$ Drainage to the contralateral axilla has been described in $14.7 \%$ of patients, in the largest available repeat SNB study. ${ }^{12}$ Aberrant drainage occurs more frequently after previous ALND, than after previous SNB. ${ }^{12}$ Therefore, CLNR could be caused by aberrant lymph drainage, especially after previous surgery of the ipsilateral breast or axilla.

It is remarkable and in line with repeat SNB studies that in this study, 18 of 20 patients (90\%) with an IBTR and synchronous CLNR, for whom information on primary axillary treatment was available, had undergone ALND. This supports the hypothesis that CLNR are regional nodal metastases of the IBTR, arising from aberrant lymph drainage after ALND. Isolated CLNR should be regarded as a different entity than an IBTR with synchronous CLNR. In this review, a difference in time to recurrence was observed between these two entities; isolated CLNRs occur significantly earlier (34 months) than IBTRs with synchronous CLNR (108 and 138 months for clinically detected and SNB detected CLNR, respectively). This suggests that isolated CLNR could be an occult contralateral nodal metastasis of the primary breast cancer, remaining in situ during the treatment of the primary breast tumour. Since the involvement of CLNs is seldom assessed in breast cancer patients, small tumour burden in a CLN would go unnoticed and untreated. Eventually, this initially subclinical disease could develop into a clinically detectable CLNR.

Although follow-up data were available for only half of all described patients, the prognosis of CLNR (82.6\% overall survival after a mean of 50.3 months) appears to be much better than the prognosis of patients with metastatic breast cancer. This prognosis 
is in line with prognosis of patients with a regional recurrence. Ipsilateral locoregionally recurrent breast cancer has a 5-year disease free survival of 56\%-84\%. ${ }^{36,37}$ The mean 5year overall survival of metastatic breast cancer varies from $23 \%$ in patients with bone metastases to only $13 \%$ in patients with visceral metastases. ${ }^{38}$ We observed some variation in overall and disease-free survival amongst different subgroups of CLNR patients, but the small numbers do not allow formal statistical testing.

Another observation from this review concerns treatment of CLNR. Although a CLNR is traditionally considered distant metastatic disease, most patients received surgical as well as systemic treatment. A total of $89.5 \%$ of patients underwent surgery for their CLNR. In patients with available data on systemic treatment, chemotherapy and/or endocrine therapy was administered in $77.8 \%$. The frequent use of surgery combined with systemic treatment implies that clinicians are treating these patients with curative rather than palliative intent, and appear to regard CLNR as a regional rather than a distant event. In addition to treatment decisions, prognosis of CLNR should have consequences for event registration in breast cancer research. For registration purposes, a composite endpoint should consist of events with similar prognostic impact, ${ }^{39}$ otherwise the clinical meaning of the endpoint is unclear. If prognosis after a CLNR differs from the prognosis of distant events, CLNRs should no longer be registered as distant metastases in breast cancer research. ${ }^{40}$

Due to the retrospective character of this study and the small number of included patients, some limitations need to be considered when interpreting the results. First, reporting bias may have occurred. Our review consists mostly of case reports and small retrospective studies. Since it might be more likely to report on remarkable cases and prognostic extremes, this may have led to both overestimation as well as underestimation of prognosis. Additionally, the small number of patients, particularly in the subgroups, is an important limitation of this study and limited comparisons of overall and disease-free survival. Also, the mean follow-up time of patients with an isolated CLNR was much longer than follow-up time of patients with IBTR and CLNR. It is important to put survival differences into the perspective of available follow-up time, since more events might occur during the course of a longer follow-up.

Despite the limitations of this study, the observed disease free survival and overall survival indicate that CLNR should be regarded as a regional rather than distant disease and should be treated accordingly. Additionally, the results indicate that in breast cancer research, CLNRs should not be registered as a distant event. Since the incidence of this phenomenon is unknown, we would suggest that CLNR should be included in a prospective registration, preferably national cancer registries, to confirm that CLNR without concurrent systemic metastases should be treated with curative intent. 


\section{References}

1. Edge SB, Compton CC. The American joint committee on cancer: the 7th edition of the AJCC cancer staging manual and the future of TNM. Ann Surg Oncol 2010;17:1471-1474.

2. Boughey JC, Ross MI, Babiera GV, et al. Sentinel lymph node surgery in locally recurrent breast cancer. Clin Breast Cancer 2006; 7:248-253.

3. Estourgie SH, Nieweg OE, Olmos RA, Rutgers EJ, Kroon BB. Lymphatic drainage patterns from the breast. Ann Surg 2004;239: 232-237.

4. Maaskant-Braat AJ, de Bruijn SZ, Woensdregt K, Pijpers H, Voogd AC, Nieuwenhuijzen GA. Lymphatic mapping after previous breast surgery. Breast 2012;21:444-448.

5. Port ER, Garcia-Etienne CA, Park J, Fey J, Borgen PI, Cody 3rd HS. Reoperative sentinel lymph node biopsy: a new frontier in the management of ipsilateral breast tumor recurrence. Ann Surg Oncol 2007;14:2209-2214.

6. Roumen RM, Kuijt GP, Liem IH. Lymphatic mapping and sentinel node harvesting in patients with recurrent breast cancer. Eur J Surg Oncol 2006;32:1076-1081.

7. Sood A, Youssef IM, Heiba SI, et al. Alternative lymphatic pathway after previous axillary node dissection in recurrent/primary breast can- cer. Clin Nucl Med 2004;29:698-702.

8. Taback B, Nguyen P, Hansen N, Edwards GK, Conway K, Giuliano AE. Sentinel lymph node biopsy for local recurrence of breast cancer after breast-conserving therapy. Ann Surg Oncol 2006;13:1099-1104.

9. Wellner R, Dave J, Kim U, Menes TS. Altered lymphatic drainage after breast-conserving surgery and axillary node dissection: local recurrence with contralateral intramammary nodal metastases. Clin Breast Cancer 2007; 7:486-488.

10. Schrenk P, Tausch C, Wayand W. Lymphatic mapping in patients with primary or recurrent breast cancer following previous axillary surgery. Eur J Surg Oncol 2008;34:851-856.

11. Tasevski R, Gogos AJ, Mann GB. Reoperative sentinel lymph node biopsy in ipsilateral breast cancer relapse. Breast 2009;18:322-326.

12. Maaskant-Braat AJ, Roumen RM, Voogd AC, et al. Sentinel node and recurrent breast cancer (SNARB): results of a nationwide registration study. Ann Surg Oncol 2013;20:620-626.

13. Agarwal A, Heron DE, Sumkin J, Falk J. Contralateral uptake and metastases in sentinel lymph node mapping for recurrent breast cancer. J Surg Oncol 2005;92:4-8.

14. Daoud J, Meziou M, Kharrat M, Sellami D, Boudawara T, Frikha M. Contralateral axillary lymph node metastasis of cancer of the breast. Bull Cancer 1998;85:713-715.

15. Herold Cl, Gaughan EM, Lamb CC, Tung NM. Second primary ipsilateral breast cancer with contralateral axillary involvement: a case report and literature review. Clin Breast Cancer 2011;11:406-408.

16. Huston TL, Pressman PI, Moore A, et al. The presentation of contralateral axillary lymph node metastases from breast carcinoma: a clinical management dilemma. Breast J 2007;13:158-164.

17. Jaffer S, Goldfarb AB, Gold JE, Szporn A, Bleiweiss IJ. Contralateral axillary lymph node metastasis as the first evidence of locally recurrent breast carcinoma. Cancer 1995;75:2875-2878.

18. Kiluk JV, Prowler V, Lee MC, Khakpour N, Laronga C, Cox CE. Contralateral axillary nodal involvement from invasive breast cancer. Breast 2014;23(3):291-294. http://dx.doi.org/10.1016/j.breast. 2014.03.004 [Epub 2014 Apr 6].

19. Kim SJ, Moon WK, Cho N, Chang JM. The detection of recurrent breast cancer in patients with a history of breast cancer surgery: comparison of clinical breast examination, mammography and ultrasonography. Acta Radiol 2011;52:15-20.

20. Kinoshita S, Hirano A, Kobayashi S, et al. Metachronous secondary primary occult breast cancer initially presenting with metastases to the contralateral axillary lymph nodes: report of a case. Breast Cancer 2010;17:71-74.

21. Koizumi M, Koyama M, Tada K, et al. The feasibility of sentinel node biopsy in the previously treated breast. Eur J Surg Oncol 2008;34: 365-368.

22. Kroon BB, Hoefnagel CA, Valdes Olmos RA, Nieweg OE. [Regional lymph nodes at a distance]. Ned Tijdschr Geneeskd 2008;152:1997-2000.

23. Lanitis S, Behranwala KA, Al-Mufti R, Hadjiminas D. Axillary metastatic disease as presentation of occult or contralateral breast cancer. Breast 2009;18:225-227. 
24. Lim I, Shim J, Goyenechea M, Kim CK, Krynyckyi BR. Drainage across midline to sentinel nodes in the contralateral axilla in breast cancer. Clin Nucl Med 2004;29:346-347.

25. Morcos B, Jaradat I, El-Ghanem M. Characteristics of and therapeutic options for contralateral axillary lymph node metastasis in breast cancer. Eur J Surg Oncol 2011;37:418-421.

26. Nishimura S, Koizumi M, Kawakami J, Koyama M. Contralateral axillary node metastasis from recurrence after conservative breast cancer surgery. Clin Nucl Med 2014;39:181-183.

27. Pasta V, Urciuoli P, D'Orazi V, Sottile D, Monti M, Redler A. Contralateral axillary metastases from breast cancer. Personal experience and review of literature. Ann Ital Chir 2014;85.

28. Sabate M, Roca I, Cordoba O, Kysiel N, Rubio I, Castell J. Contralateral axillary drainage in breast tumor recurrence. Rev Esp Med Nucl 2011;30:327-328.

29. Schlechter BL, Yang Q, Larson PS, et al. Quantitative DNA fingerprinting may distinguish new primary breast cancer from disease recurrence. J Clin Oncol 2004;22:1830-1838.

30. Tokmak H, Kaban K, Muslumanoglu M, Demirel M, Aktan S. Management of sentinel node re-mapping in patients who have second or recurrent breast cancer and had previous axillary procedures. World J Surg Oncol 2014;12:205.

31. van der Ploeg IM, Oldenburg HS, Rutgers EJ, et al. Lymphatic drainage patterns from the treated breast. Ann Surg Oncol 2010;17: 1069-1075.

32. Suami H, Pan WR, Taylor GI. Historical review of breast lymphatic studies. Clin Anat 2009;22:531-536.

33. Barranger E, Montravers F, Kerrou K, et al. Contralateral axillary sentinel lymph node drainage in breast cancer: a case report. J Surg Oncol 2004;86:167-169.

34. Lizarraga IM, Scott-Conner CE, Muzahir S, Weigel RJ, Graham MM, Sugg SL. Management of contralateral axillary sentinel lymph nodes detected on lymphoscintigraphy for breast cancer. Ann Surg Oncol 2013;20:3317-3322.

35. Trifiro G, Ravasi L, Paganelli G. Contralateral or bilateral lymph drainage revealed by breast lymphoscintigraphy. Eur J Nucl Med Mol Imaging 2008;35:225-229.

36. Clemons M, Danson S, Hamilton T, Goss P. Locoregionally recurrent breast cancer: incidence, risk factors and survival. Cancer Treat Rev 2001;27:67-82.

37. Shenouda MN, Sadek BT, Goldberg SI, et al. Clinical outcome of isolated locoregional recurrence in patients with breast cancer according to their primary local treatment. Clin Breast Cancer 2014;14: 198-204.

38. Giordano SH, Buzdar AU, Smith TL, Kau SW, Yang Y, Hortobagyi GN. Is breast cancer survival improving? Cancer 2004; 100:44-52.

39. Montori VM, Permanyer-Miralda G, Ferreira-Gonzalez I, et al. Validity of composite end points in clinical trials. BMJ 2005;330:594-596.

40. Moossdorff M, Van Roozendaal L, Strobbe LJA, et al. Maastricht Delphi Consensus on event definitions for classification of recurrence in breast cancer research. J Natl Cancer Inst 2014;106(12). http://dx.doi.org/10.1093/jnci/dju288 pii: dju288. Print 2014. 


\section{Appendix 3.1}

(((contralateral[All Fields] OR (contralateral[All Fields] AND ("axilla"[MeSH Terms] OR "axilla"[All Fields]))) OR (contralateral[All Fields] AND ("axilla"[MeSH Terms] OR "axilla”[All Fields]))) AND ((((("Iymph nodes"[MeSH Terms] OR ((“Iymph"[MeSH Terms] OR "lymph"[All Fields]) AND nodes[All Fields])) OR ("lymph nodes"[MeSH Terms] OR ("Iymph"[All Fields] AND "no- des"[All Fields]) OR "lymph nodes"[All Fields])) OR (("Iymph"[MeSH Terms] OR "Iymph"[All Fields]) AND node[All Fields])) OR ("Iymph nodes"[MeSH Terms] OR ("Iymph"[All Fields] AND "nodes"[All Fields]) OR "lymph nodes"[All Fields] OR ("lymph"[All Fields] AND "node"[All Fields]) OR "lymph node"[All Fields])) OR "Lymph Nodes"[Mesh])) AND (((((((“breast neoplasms"[- MeSH Terms] OR "breast neoplasms"[MeSH Terms]) OR ("breast neoplasms"[MeSH Terms] OR ("breast"[All Fields] AND "neoplasms"[All Fields]) OR "breast neo- plasms"[All Fields])) OR (("breast"[MeSH Terms] OR "breast"[All Fields]) AND ("neoplasms"[MeSH Terms] OR "neoplasms"[All Fields]))) OR (("breast"[MeSH Terms] OR "breast"[All Fields]) AND ("neoplasms"[MeSH Terms] OR "neoplasms"[All Fields] OR "cancer"[- All Fields]))) OR ("breast neoplasms"[MeSH Terms] OR ("breast"[All Fields] AND "neoplasms"[All Fields]) OR "breast neoplasms"[All Fields] OR ("breast"[All Fields] AND "cancer"[All Fields]) OR "breast cancer"[All Fields])) OR (("breast"[MeSH Terms] OR "breast"[All Fields]) AND ("carcinoma"[MeSH Terms] OR "carcino- ma"[All Fields]))) OR ("breast neoplasms"[MeSH Terms] OR ("breast"[All Fields] AND "neoplasms"[All Fields]) OR "breast neoplasms"[All Fields] OR ("breast"[All Fields] AND "carcinoma"[All Fields]) OR "breast carcinoma"[All Fields])). 



\section{Chapter 4}

Prognosis of contralateral lymph node recurrence: data from national cancer registries and individual hospitals

Moossdorff M, Vugts G, Kühn T, de Munck L, Filtenborg Tvedskov T, Leidenius M, Rubio IT, Kroman N, Nieuwenhuijzen GAP, Stassen LPS, Smidt ML 


\section{Abstract}

\section{Introduction}

After curative treatment for breast cancer, some patients experience a recurrence in a contralateral lymph node (CLNR). At initial diagnosis, these are traditionally considered distant events. However, after treatment of breast cancer, aberrant lymph drainage is common and CLNR may actually be the first nodal basin, suggesting a regional event. This study aims to determine prognosis after CLNR compared to ipsilateral lymph node recurrence (ILNR) and distant metastasis (DM).

\section{Methods}

Cases of CLNR were identified in two national cancer databases and three individual hospital databases. Endpoints were overall survival (OS) and breast cancer specific survival (BCSS). Results were presented separately for different eras of diagnosis. For comparison, OS of ILNR and DM were calculated.

\section{Results}

A total of 183 cases of CLNR were identified. Median age at initial diagnosis was 56 years. Year of initial diagnosis was 2005 or later in 51 patients (27.9\%), 1995-2004 in 46 (25.1\%), and before 1995 in 85 (47.0\%). Median time to CLNR was 25 months. Median follow-up after CLNR was 26 months. Five year OS was 30.2\%, this was slightly better for more recent years of initial diagnosis (<1995: 19.8\%; 1995-2004: 46.1\%; >2005: 33.6\%). BCSS data was available for 158 cases and 5-year BCSS was $33.4 \%$. Five year OS after ILNR ( $n=75,2005-2008$ Dutch cohort only) was $57.4 \%$ and $10.1 \%$ after DM $(n=2748)$.

\section{Conclusion}

OS after CLNR was poor at 30.2\% after 5 years, BCSS was similar to OS. Patients diagnosed more recently had slightly better prognosis. Although this study is at risk for underestimation of prognosis, it suggests that prognosis is worse than after ILND although slightly better than after DM. Despite poor prognosis compared to ILNR, treatment with curative intent may be suitable for individual patients. 


\section{Introduction}

After treatment for breast cancer, some patients experience recurrence in a contralateral lymph node (CLNR). When positive contralateral lymph nodes are encountered at initial diagnosis, they are traditionally considered distant metastases. ${ }^{1}$ However, after breast cancer treatment, aberrant lymph drainage is common, particularly after radiotherapy and axillary surgery. ${ }^{2-10}$ This may mean that contralateral lymph nodes are actually the first basins that lymph from the treated breast drains towards, and CLNR are therefore more similar to ipsilateral lymph node recurrences (ILNR, usually considered regional recurrences) than distant metastasis.

Although CLNR is a rare entity, we may encounter it more frequently in the future. CLNR can be detected clinically as a palpable node, but also during workup for an ipsilateral breast tumor recurrence (IBTR), for instance by PET(-CT) or repeat sentinel node procedure. As these are increasingly used, we might detect more CLNR than previously.

The meaning of CLNR in terms of prognosis influences the approach to an individual patient experiencing CLNR, and also the classification of CLNR in breast cancer research: do we count them as regional recurrences or as distant disease?

A review of all published cases and case series ${ }^{11}$ suggested that after a median follow-up of 50.3 months, overall survival was $82.6 \%$ (95\% $\mathrm{Cl}$ 67.1-98.1) and disease free survival was $65.2 \%$ (45.7-84.7). Five year overall survival of metastatic breast cancer varies but is reported to be $23 \%$ in patients with bone metastases and $13 \%$ in patients with visceral metastases. ${ }^{12}$ Prognosis of CLNR in this review was better than the expected prognosis of distant metastasis and suggests that CLNR is more similar to regional recurrence. However, heterogeneous data and a small number of subjects limited this review.

The aim of this study is to estimate the prognosis of CLNR in a more homogenous and larger population to determine whether it should be seen as a regional or a distant recurrence.

\section{Methods}

Retrospective data on the occurrence and prognosis of CLNR were collected from two national databases as well as from individual hospital databases (Table 4.1). Local collaborating physicians who obtained the data from patient's records supplied information from individual hospitals. Data were provided without patient identifiers in a secure file format. The local institutional review board waived the need for medical 
ethical approval and informed consent of patients, as information could not be traced back to individual persons.

\section{Selection of patients}

Inclusion criteria were patients 18 years of age or over, with a previous history of curative treatment for invasive breast cancer, with pathology confirmed breast cancer recurrence in a contralateral lymph node (i.e. contralateral axillary, internal mammary, supra- or infraclavicular or intramammary lymph node). The CLNR can be either isolated (i.e. in absence of ipsilateral breast recurrence), or synchronous with an IBTR.

Exclusion criteria were objection of the patient to use data for research purposes, history of bilateral breast cancer, synchronous distant metastases, and synchronous contralateral breast cancer (on the side of the current contralateral lymph node, i.e. the other breast than where the original tumor was located).

\section{Collected data}

Patients were identified by searching national databases. The first database was the Netherlands Cancer Registry (Comprehensive Cancer Organisation the Netherlands, IKNL). Trained data registration clerks obtained the data from patients' charts from all hospitals in the Netherlands. For a period of 5 years after diagnosis, the first breast cancer event was registered for a total of 34453 breast cancer patients. Survival status was derived from the Dutch population register and up to date until December 31, 2013. The second database was the Danish Breast Cancer registry (DBCG). Survival status was derived from the Danish Population Registry and available until June 15, 2015 and cause of death until December 31, 2013.

Individual cases were obtained from hospital databases from three hospitals: Klinikum Esslingen (Esslingen, Germany), Helsinki University Hospital (Helsinki, Finland), and Hospital Universitario Vall d'Hebron (Barcelona, Spain). Data were collected prospectively but not specifically for this purpose.

If available, the following types of data were collected: patient age, characteristics of the primary tumor and its treatment including specific treatment to the axilla, characteristics of the CLNR (with or without concurrent IBTR) and its treatment, including detection method, location and number of positive nodes, distant events after CLNR, survival, and cause of death.

\section{Outcomes and statistics}

The primary endpoint was overall survival (OS, defined as time from CLNR to death of any cause), and breast cancer specific survival (BCSS, time from CLNR to death resulting 
from breast cancer). OS results are presented separately for different cohorts of year of diagnosis. BCSS status (i.e. cause of death) was not registered for the Dutch cancer registry population. As these missing values are not random and the Dutch database formed a significant proportion of the total study population, including them (and treating them either as breast cancer deaths or non-breast cancer deaths) would distort the results. Therefore, all cases from the Dutch cancer registry (both surviving and deceased subjects) were excluded from the BCSS analysis.

Analyses were performed using SPSS [IBM Corporation, version 23.0.0.0]. Kaplan-Meier analysis was used to determine OS and BCSS after 24 and 60 months after CLNR.

\section{Comparison with prognosis after ILNR and DM}

For comparison, OS after ILNR and DM were determined from the Netherlands Cancer Registry database. Data on ILNR and DM were not available from the other data sources. For the ILNR analysis, cases with synchronous DM (i.e. <91 days of initial diagnosis) were excluded in analogy to the CLNR analyses. For calculating OS after DM, synchronous other events were not excluded.

\section{Results}

\section{Data sources}

Two cancer registries and three individual hospitals participated. Characteristics are shown in Table 4.1.

\section{Table 4.1 Data sources}

\begin{tabular}{lccc}
\hline Source & $\mathrm{N}=$ & Year of initial diagnosis & Outcomes available \\
\hline Dutch national cancer registry (IKNL) & $25^{*}$ & $2005-2008$ & OS \\
Danish national cancer registry & 152 & $1978-2012$ & OS, BCSS \\
Helsinki University Hospital, Finland & 2 & $2000-2002$ & OS, BCSS, DM \\
Klinikum Esslingen, Germany & 2 & 2012 & OS, BCSS, DM \\
Hospital Universitario Vall d'Hebron, Spain & 2 & $1999-2001$ & OS, BCSS, DM \\
\hline
\end{tabular}

* Total number of patients with complete 5 -year follow up: $n=34453$, i.e. 0,07\%

OS: overall survival, BCSS: breast cancer specific survival, DM: distant metastasis

\section{Baseline characteristics}

A total of 183 cases of CLNR after breast cancer treatment were available from these sources. The years of diagnosis per source are listed in Table 4.1. In total, 51 patients (27.9\%) were diagnosed in or after 2005, 46 (25.1\%) between 1995 and 2004, and 85 
(47.0\%) were diagnosed before 1995. Patient and tumor characteristics are shown in Table 4.2. Median age at initial breast cancer diagnosis was 56 years. Most tumors were pT1 and pT2 and only 41 (22.4\%) were pNO at initial diagnosis. Receptor status was unknown for a considerable number of patients. ER was known for 111 patients (60.7\%), of which 75 were ER+ (67.5\%, 41\% of total). Her2 status was known for 61 (33.3\%) patients, of which 17 were Her2+ (27.9\%, 9.3\% of total). Mastectomy was performed in $153(83.6 \%)$ of patients. This percentage was slightly higher in patients diagnosed earlier; i.e. 94.2\% when diagnosed before 1995, 73.9\% when diagnosed between 1995 and 2004, and $74.5 \%$ when diagnosed from 2005.

The median time from diagnosis to CLNR was 25 months (mean 38 months, range 0.7-264). Location and detection method of the CLNR was unfortunately unknown in the majority of cases (170/183, 92.9\%), as was the number of affected nodes (175/183, 95.6\%).

Treatment after CLNR was also unknown for a large number of subjects. In the cases with complete data on treatment, surgery of the affected lymph nodes was performed in $19 / 34$ (55.9\%) of subjects, radiation therapy was performed in 16/36 (44.4\%), chemotherapy was administered to $18 / 35$ (51.4\%) and endocrine therapy to $16 / 36$ (44.4\%).

\section{Survival analysis}

The median follow-up after diagnosis of CLNR for OS was 26.3 months (mean 44.0 months, range 2.4-346.3). Median follow-up was 39.9 months (mean 38.8) in the Dutch database, 26.2 months (mean 45.3) in the Danish database, and 25.3 months (mean 31.0 ) in the cases from the individual hospitals. OS data was complete for all 183 cases. After 24 months, OS was 58.2\% and after 60 months (i.e. 5 years), OS was 30.2\% (Figure 4.1a).

For BCSS (Figure 1b), 25 patients from the Dutch National Cancer Registry were excluded (see Methods section). Subsequently, BCSS data were available for 158 subjects, in which median follow-up was 26.2 months (mean 44.8, range 2.4-346.3). By exclusion of the Dutch Cancer registry patients, the subjects included in the BCSS analysis were diagnosed earlier, namely before 1995 in 86 of patients (54.4\%), between 1995 and 2004 in 46 (29.1\%), and in 2005 or later in 26 (16.5\%). Of the included patients, 29 (18.4\%) were alive at last follow up, 110 (69.6\%) died of breast cancer, 6 (3.8\%) died of another cancer, 7 (4.4\%) died of an other cause, and $6(3.8 \%)$ died of an unknown cause. Survival analysis revealed that after 24 months, BCSS was $60.0 \%$ and after 60 months, BCSS was $33.4 \%$. 
Table 4.2 Patient, tumor, and treatment characteristics

\begin{tabular}{|c|c|c|c|c|c|}
\hline Age at initial & Median (range) & $56(26-87)$ & Initial chemotherapy & Yes & $79(43.2 \%)$ \\
\hline diagnosis & & & & No & $46(25.1 \%)$ \\
\hline Initial breast & pT1 & $71(38.8 \%)$ & & Unknown & $58(31.7 \%)$ \\
\hline cancer pT & pT2 & $74(40.4 \%)$ & & & \\
\hline & pT3 & $27(14.8 \%)$ & Initial endocrine & Yes & $67(36.6 \%)$ \\
\hline & pT4 & $4(2.2 \%)$ & therapy & No & $58(31.7 \%)$ \\
\hline & Unknown & $7(3.8 \%)$ & & Unknown & $58(31.7 \%)$ \\
\hline Initial breast & pNO & $41(22.4 \%)$ & Initial trastuzumab & Yes & $8(4.4 \%)$ \\
\hline cancer pN & pN1 & $63.9 \%(63.9 \%)$ & & No & $117(63.9 \%)$ \\
\hline & pN2 & $7(3.8 \%)$ & & Unknown & $58(31.7 \%)$ \\
\hline & pN3 & $12(6.6 \%)$ & & & \\
\hline Initial tumor & $\mathrm{ER+}$ & 75 (41\%) & & & \\
\hline receptors & ER unknown & $72(39.3 \%)$ & & & \\
\hline & $\mathrm{PR}+$ & $42(23 \%)$ & & & \\
\hline & PR unknown & $101(55 \%)$ & Characteristics of CLN & $\mathrm{R}$ and its treatme & ent \\
\hline & Her2+ & $17(9.3 \%)$ & $\begin{array}{l}\text { Time from initial } \\
\text { diagnosis to CLNR }\end{array}$ & $\begin{array}{l}\text { Months, } \\
\text { median (range) }\end{array}$ & $25(0.7-264)$ \\
\hline & Her2 unknown & $122(66.7 \%)$ & With concurrent & Yes & $9(15.8 \%)$ \\
\hline Initial histology & Ductal & $149(81.3 \%)$ & IBTR & No & $29(4.9 \%)$ \\
\hline & Lobular & $18(9.8 \%)$ & & Unknown & $145(79.2 \%)$ \\
\hline & Medullary & $4(2.2 \%)$ & CLNR surgery & Yes & 19 (10.4\%) \\
\hline & Other & $5(2.7 \%)$ & & No & $15(8.7 \%)$ \\
\hline & Unknown & $2(1 \%)$ & & Unknown & $148(80.9 \%)$ \\
\hline Initial breast & Mastectomy & $153(83.6 \%)$ & CLNR radiation & Yes & $16(8.7 \%)$ \\
\hline cancer surgery* & BCS & $30(16.4 \%)$ & therapy & No & $20(10.9 \%)$ \\
\hline Initial axillary & ALND & $111(60.7 \%)$ & & Unknown & $147(80.3 \%)$ \\
\hline surgery* & SN only & $5(2.7 \%)$ & CLNR chemotherapy & Yes & $18(9.8 \%)$ \\
\hline & Nodal sampling ${ }^{\#}$ & $64(35.0 \%)$ & & No & $17(9.3 \%)$ \\
\hline & Unknown & $3(1.6 \%)$ & & Unknown & $148(80.9 \%)$ \\
\hline Initial radiation & Yes & $64(35 \%)$ & CLNR endocrine & Yes & $16(8.7 \%)$ \\
\hline therapy & No & $5(2.7 \%)$ & therapy & No & 20 (10.9\%) \\
\hline & Unknown & $114(62.3 \%)$ & & Unknown & $147(80.3 \%)$ \\
\hline
\end{tabular}

ER estrogen receptor, PR progesterone receptor, BCS breast conserving surgery, ALND axillary lymph node dissection, SN sentinel node procedure, IBTR ipsilateral breast tumor recurrence, CLNR contralateral lymph node recurrence. ${ }^{*}$ most extensive surgery listed, if first SNB and then completion ALND, or first BCS and then mastectomy, only ALND and mastectomy are counted respectively. \# nodal sampling was performed in the Danish study population

\section{Prognosis depending on year of diagnosis}

Particularly the Danish database included a large number of patients who were initially diagnosed several decades ago. OS in categories depending on year of initial breast cancer diagnosis is shown in Figure 4.2. OS after 24 and 60 months for patients diagnosed in 2005 or later were $72.4 \%$ and $33.6 \%$ respectively. For patients diagnosed between 1995 and 2004, 24 month OS was 73.4\% and 60 month OS 46.1\%. In patients diagnosed before 1995, OS 24 months after CLNR was 43.0\%, and 19.8\% after 60 months. 


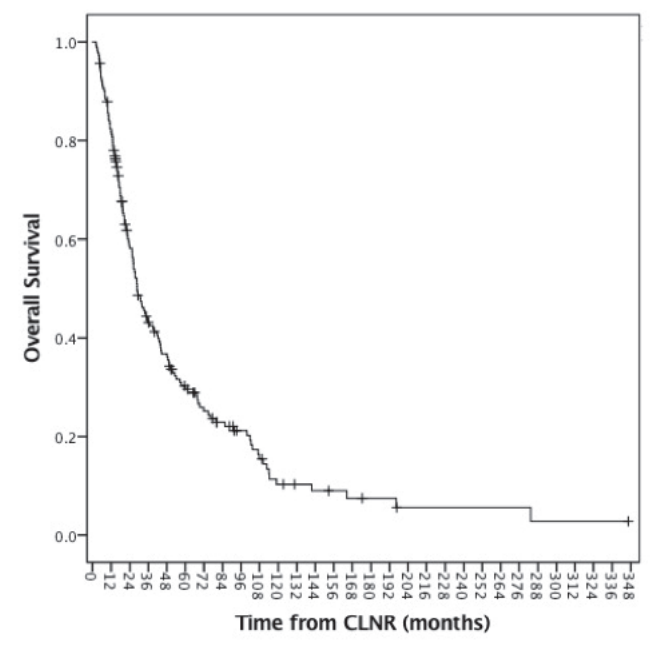

\begin{tabular}{lccccc}
\hline Months & 0 & 12 & 24 & 60 & 120 \\
\hline No. at risk & 183 & 149 & 97 & 44 & 10 \\
\hline
\end{tabular}

Figure 4.1a Kaplan-Meier estimator plots of overall survival after CLNR (from time of CLNR to death or end of follow-up)

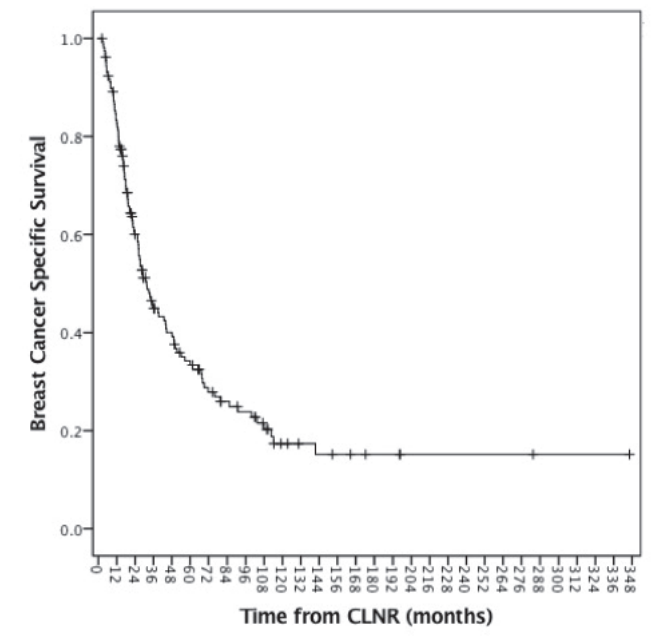

\begin{tabular}{lccccc}
\hline Months & 0 & 12 & 24 & 60 & 120 \\
\hline No. at risk & 158 & 128 & 82 & 40 & 10 \\
\hline
\end{tabular}

Figure 4.1b Kaplan-Meier estimator plots of breast cancer specific survival after CLNR (from time of CLNR to death or end of follow-up) 


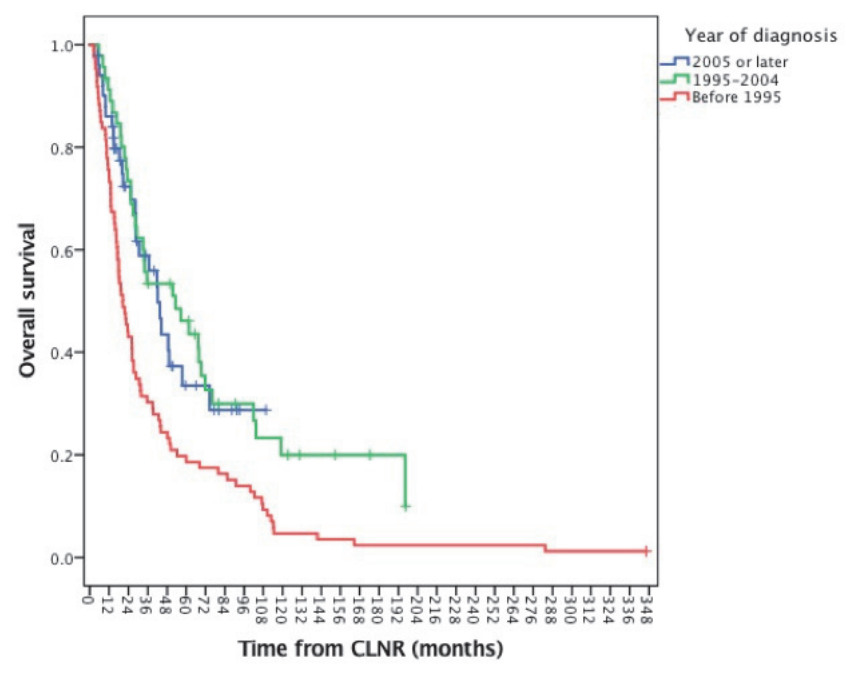

\begin{tabular}{llllrl}
\hline Months & 0 & 12 & 24 & 60 & 120 \\
\hline No. at risk & & & & & \\
\hline$>2005$ & 51 & 43 & 27 & 8 & 0 \\
\hline $1995-2004$ & 46 & 41 & 33 & 19 & 6 \\
\hline$<1995$ & 89 & 65 & 37 & 17 & 4 \\
\hline
\end{tabular}

Figure 4.2 Kaplan-Meier estimator plots of overall survival in different eras of initial breast cancer diagnosis (from time of CLNR to death or end of follow-up)

\section{Prognosis after ILNR and distant metastases}

From the Netherlands Cancer Registry database (containing patients diagnosed between 2005 and 2008), 75 (/34453, 0.2\%) cases of ILNR without simultaneous distant metastases were identified. Median time to ILNR was 23.1 months (range 2.8-59.4). Median follow-up after ILNR was 45.9 months (7.9-93.5). Overall survival was $73.3 \%$ after 2 years $57.4 \%$ after 5 years (Figure 4.3a).

From the same database, 2948 (/34453, 8.5\%) cases of distant metastases as a first event were identified. Median time to distant metastasis was 26.2 months (range 3.0-60.2). Median follow-up after DM was 17.1 months (range 3.0-112.7). Overall survival was $38.5 \%$ after 2 years and $10.3 \%$ after 5 years (Figure $4.3 \mathrm{~b}$ ). 
a

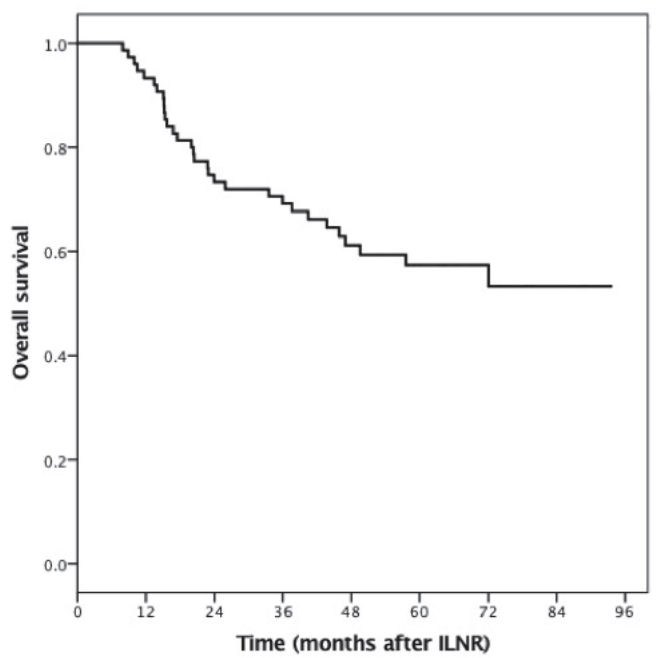

\begin{tabular}{lcccc}
\hline Months & 0 & 12 & 24 & 60 \\
\hline No. at risk & 75 & 70 & 51 & 25 \\
\hline
\end{tabular}

b

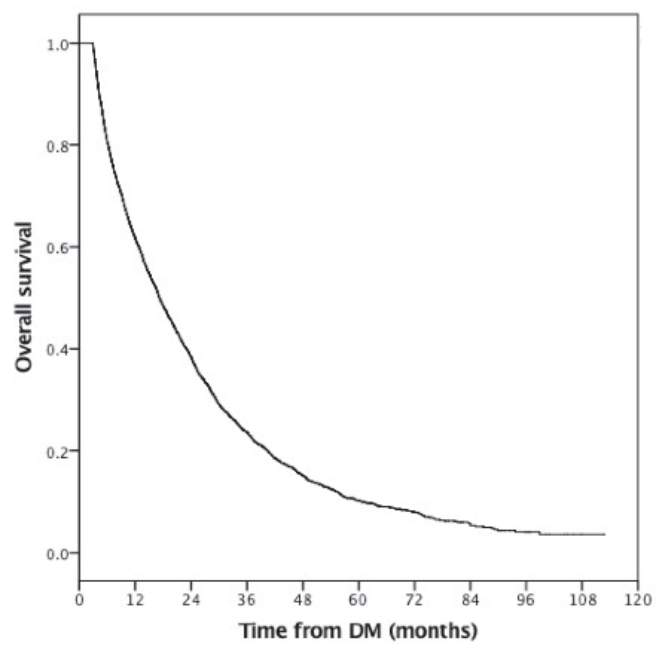

\begin{tabular}{lccccc}
\hline Months & 0 & 12 & 24 & 60 & 96 \\
\hline No. at risk & 2948 & 1819 & 1113 & 197 & 13 \\
\hline
\end{tabular}

Figure 4.3 Kaplan-Meier estimator plots of overall survival after ILNR (a) and distant metastasis (b) (from time of ILNR/DM until time of death or end of follow-up) 


\section{Discussion}

This study, investigating prognosis after breast cancer recurrence in a contralateral lymph node (without simultaneous distant metastases), in 183 patients from two national cancer registries and three individual hospital databases, shows that 5 year OS after CLNR is poor with $30.2 \%$ (compared to $57.4 \%$ after ipsilateral lymph node recurrence and $10.1 \%$ after distant metastasis, from the Dutch National Cancer Registry from 2005-2008). More recent diagnosis of initial breast cancer showed slightly better prognosis: 5-year OS was 19.8\% when diagnosed before 1995, 46.1\% when diagnosed between 1995 and 2004, and 33.6\% when diagnosed after 2005. BCSS was similar to OS, although BCSS data was available mainly for patients who were diagnosed in earlier decades. Although information on local and systemic treatment of CLNR and presence of simultaneous ipsilateral breast tumor recurrence was missing and results could not be corrected for these factors, this study suggests that prognosis of CLNR is inferior compared to ILNR although slightly better than prognosis after DM, in contrast to earlier publication.

The major strength of this study is that it is the largest compilation of information on prognosis of CLNR without simultaneous (other) distant metastases to date. Limitations concern mainly missing data from the various data sources. The Dutch database was limited by lacking information on cause of death. As a result, these patients could not be included in the BCSS analysis. As the Dutch database contained relatively recent cases, this may lead to underestimation of BCSS. The Danish database included patients who were diagnosed as early as in the 1970s, which means both initial treatment and treatment of the CLNR itself (particularly systemic) may be suboptimal to current standards which may lead to underestimation of prognosis in this study. It also made it harder to validate the absence of simultaneous distant metastasis, both in terms of registration and limited diagnostics at the time of CLNR diagnosis.

Compared to the earlier published systematic review on this subject ${ }^{11}$, this study shows inferior prognosis. The earlier review found an OS of $82.6 \%$ after a median follow up of 50.3 months, which is even higher than the observed OS in ILNR. This may be explained by the fact that this review included case reports that may be subject to publication bias of favorable results. A second explanation may be that $47 \%$ of patients in the present study were diagnosed before 1995. Although this may usually bias towards underestimation of prognosis, the separate analysis of patients diagnosed after 2005 showed slightly better prognosis compared to earlier cohorts, but still inferior to prognosis after ILNR which was derived from the same years of diagnosis (5-year OS 
$33.6 \%$ vs 57.3\%). Furthermore, many cases in the earlier published review were derived from repeat sentinel node studies, and it was already suggested that CLNR with IBTR has more favorable prognosis than isolated CLNR. Due to missing data (only 9 cases with known IBTR+CLNR, unknown for 145 subjects), this could not be explored further in the current study and an overrepresentation of isolated CLNR may have lead to inferior prognosis. Finally, data on treatment after CLNR (local and systemic) were missing for an important part of the study population. As a result, we could not explore whether patients were treated with curative intent and how this affected prognosis.

In summary, this study shows that CLNR has a 5-year OS of 30\%, which is inferior compared to ILNR but better than distant disease in the current era. In literature, ipsilateral locoregional recurrence (breast and/or lymph nodes) has a 5-year DFS of 5684\%. ${ }^{13-15}$ The randomized CALOR trial included patients with completely excised isolated locoregional recurrence, and showed 5-year DFS of 69\% (56-79\%) with chemotherapy and $57 \%$ (44-67\%) without chemotherapy. ${ }^{16}$ These outcomes are similar to 5-year OS after ILNR (with/without ipsilateral breast tumor recurrence) that was calculated for comparison in the current study. In contrast, it has been described earlier that "locoregional recurrence outside the breast" carries a far worse outcome with 5-year OS of $24.1 \% .{ }^{15}$ Prognosis of distant metastases are associated with even lower 5 -year OS, for instance $23 \%$ for bone and $13 \%$ for visceral metastases ${ }^{12}$, similar to 5 -year OS after DM that was observed in the current study.

The current study suggests that prognosis after CLNR is inferior to prognosis after ILNR, although better than prognosis after DM, notwithstanding a potential risk for underestimation of prognosis in this study. Future evidence on recent cohorts may illuminate this issue further, including the difference between isolated CLNR and CLNR with synchronous IBTR. For instance, repeat sentinel node studies could prospectively evaluate prognosis of the patients in which CLNR were present. Up until then, we suggest that CLNR is classified as a distant event in breast cancer research. However, in clinical practice, it is conceivable that physicians determine to treat with curative intent depending on the individual patient (e.g. with resection and for instance systemic therapy, as suggested by the CALOR trial for isolated locoregional recurrences ${ }^{16}$ ). 


\section{References}

1 Edge SB, Compton CC. The American Joint Committee on Cancer: the 7th edition of the AJCC cancer staging manual and the future of TNM. Ann Surg Oncol. 2010;17:1471-1474.

2 Boughey JC, Ross MI, Babiera GV, Bedrosian I, Feig BW, Hwang RF, et al. Sentinel lymph node surgery in locally recurrent breast cancer. Clin Breast Cancer. 2006;7:248-253.

3 Estourgie SH, Nieweg OE, Olmos RA, Rutgers EJ, Kroon BB. Lymphatic drainage patterns from the breast. Ann Surg. 2004;239:232-237.

4 Maaskant-Braat AJ, de Bruijn SZ, Woensdregt K, Pijpers H, Voogd AC, Nieuwenhuijzen GA. Lymphatic mapping after previous breast surgery. Breast. 2012;21:444-448.

5 Port ER, Garcia-Etienne CA, Park J, Fey J, Borgen PI, Cody HS3. Reoperative sentinel lymph node biopsy: a new frontier in the management of ipsilateral breast tumor recurrence. Ann Surg Oncol. 2007;14: 2209-2214.

6 Roumen RM, Kuijt GP, Liem IH. Lymphatic mapping and sentinel node harvesting in patients with recurrent breast cancer. Eur J Surg Oncol. 2006;32:1076-1081.

7 Sood A, Youssef IM, Heiba SI, El-Zeftawy H, Axelrod D, Seigel B, et al. Alternative lymphatic pathway after previous axillary node dissection in recurrent/primary breast cancer. Clin Nucl Med. 2004;29:698-702.

8 Taback B, Nguyen P, Hansen N, Edwards GK, Conway K, Giuliano AE. Sentinel lymph node biopsy for local recurrence of breast cancer after breast-conserving therapy. Ann Surg Oncol. 2006;13:1099-1104.

9 Wellner R, Dave J, Kim U, Menes TS. Altered lymphatic drainage after breast-conserving surgery and axillary node dissection: local recurrence with contralateral intramammary nodal metastases. Clin Breast Cancer. 2007;7:486-488.

10 Vugts G, Maaskant-Braat AJG, Voogd AC, van Riet YEA, Luiten EJT, Rutgers EJT, et al. Repeat sentine node biopsy should be considered in patients with locally recurrent breast cancer. Breast Cancer Res Treat. 2015;153:549-556.

11 Moossdorff M, Vugts G, Maaskant-Braat AJ, Strobbe LJ, Voogd AC, Smidt ML, et al. Contralateral lymph node recurrence in breast cancer: Regional event rather than distant metastatic disease. A systematic review of the literature. Eur J Surg Oncol. 2015;41:1128-1136.

12 Giordano SH, Buzdar AU, Smith TL, Kau SW, Yang Y, Hortobagyi GN. Is breast cancer survival improving? Cancer. Wiley Subscription Services, Inc., A Wiley Company; 2004;100:44-52.

13 Clemons M, Danson S, Hamilton T, Goss P. Locoregionally recurrent breast cancer: incidence, risk factors and survival. Cancer Treat Rev. 2001;27:67-82.

14 Shenouda MN, Sadek BT, Goldberg SI, Keruakous AR, Croft BJ, Abi Raad RF, et al. Clinical outcome of isolated locoregional recurrence in patients with breast cancer according to their primary local treatment. Clin Breast Cancer. 2014;14:198-204.

15 Wapnir IL, Aebi S, Geyer CE, Zahrieh D, Gelber RD, Anderson SJ, et al. A Randomized Clinical Trial of Adjuvant Chemotherapy for Radically Resected Locoregional Relapse of Breast Cancer: IBCSG 27-02, BIG 1-02, and NSABP B-37. Clin Breast Cancer. 2008;8:287-292.

16 Aebi S, Gelber S, Anderson SJ, Lang I, Robidoux A, Martin M, et al. Chemotherapy for isolated locoregional recurrence of breast cancer (CALOR): a randomised trial. Lancet Oncol. 2014;15:156-163. 



\section{Chapter 5}

\section{TNM classification and the need for revision of pN3a breast cancer}

van Nijnatten TJA, Moossdorff M, de Munck L, Goorts B, Vane MLG, Keymeulen KBMI, Beets-Tan RGH, Lobbes MBI, Smidt ML

Eur J Cancer 2017;79:23-30 


\section{Abstract}

\section{Background}

According to the seventh edition of tumour-node-metastasis (TNM) classification, pN3a status in breast cancer patients consists of presence of an infraclavicular lymph node metastasis (LNM) and/or presence of $\geq 10$ axillary LNMs. The aim of this study was to determine whether prognosis of pN3a based on at least an infraclavicular LNM differs from $\geq 10$ axillary LNMs.

\section{Methods}

Data were obtained from the Netherlands Cancer Registry. All patients were diagnosed between 2005 and 2008 with primary invasive epithelial breast cancer and pN2a or pN3a status as pathologic result. Patients with pN3a were subdivided in pN3a based on at least an infraclavicular LNM or $\geq 10$ axillary LNMs. Disease-free survival (DFS) included any local, regional or contralateral recurrence, distant metastasis or death within 5 years. Kaplan-Meier curves provided information on 5-year DFS and 8-year overall survival (OS). In addition, Cox proportional hazards model was used to measure the effect of relevant clinicopathological variables on DFS and OS.

\section{Results}

A total of 3400 patients with pN2a and 1788 patients with pN3a were included. In 83 patients, pN3a was based on at least an infraclavicular LNM (4.6\%) and in 1705 patients because of $\geq 10$ axillary LNMs (95.4\%). After multivariable analyses, DFS and OS were inferior in patients with pN3a based on $\geq 10$ axillary LNMs compared to infraclavicular LNM (DFS 48.8\% versus 63.8\%, hazard ratio [HR] 1.59, $p=0.036$; OS 46.6\% versus $63.9 \%, H R$ 1.46, $p=0.042$ ). Furthermore, $p N 2 a$ and $p N 3 a$ based on infraclavicular LNM had comparable DFS and OS.

\section{Conclusion}

PN3a status based on an at least an infraclavicular LNM is rare, yet its prognosis is superior to $\geq 10$ axillary LNMs. Reclassification of infraclavicular LNM in the next TNM should therefore be considered into pN2a. 


\section{Introduction}

In 1958, the first edition of the tumour-node-metastasis (TNM) classification of malignant tumours of the breast was published by the Union for International Cancer Control (UICC) in order to achieve worldwide consensus for the classification of, eventually, each solid tumour type. ${ }^{1}$ Subsequently, this classification system was revised each decade to implement new insights. For instance, the introduction of neoadjuvant systemic therapy, sentinel lymph node biopsy, immunohistochemical staining and the method of pathologic nodal staging. ${ }^{2}$

Regarding pathologic nodal staging, axillary lymph node metastases (LNMs) were divided into three categories in the fifth edition of the TNM classification: pNO (0 axillary LNMs), pN1 (movable axillary LNMs) and pN2 (fixed axillary LNMs). ${ }^{3}$ After revision in sixth edition, the number of axillary LNMs was incorporated as key element in the classification, as impaired prognosis was demonstrated in the presence of an increasing number of axillary LNMs. ${ }^{4}$ This resulted in four categories: pNO (0 axillary LNMs), pN1a (1-3 axillary LNMs), pN2a (4-9 axillary LNMs) and pN3a ( $\geq 10$ axillary LNMs). ${ }^{5}$ Furthermore, a study by Newman et al. observed a worse disease-free (DFS) and overall survival (OS) in patients with infraclavicular (level III) and axillary LNMs compared to patients with axillary LNMs only (DFS 50\% versus 68\%; OS 58\% versus 83\%, respectively). ${ }^{6}$ As a consequence, the UICC decided to redefine infraclavicular LNM as pN3a in the sixth edition; in contrast to earlier, when an infraclavicular LNM was considered equivalent to other axillary LNMs in the fifth edition. Currently pN3a nodal status consists of patients with $\geq 10$ axillary LNMs and of patients with infraclavicular LNM. $^{7}$

The combination of both groups within pN3a suggests that their prognosis is similar. ${ }^{8,9}$ However, no study thus far analysed this assumption. Therefore, the purpose of this study is to determine whether the prognosis of pN3a breast cancer patients based on at least an infraclavicular LNM is different compared to patients with $\geq 10$ axillary LNMs and to patients with 4-9 axillary LNMs.

\section{Materials and methods}

\section{Data collection}

Data were obtained from the Netherlands Cancer Registry (NCR), managed by the Netherlands Comprehensive Cancer Organisation (IKNL). The NCR collects data of all patients diagnosed with any type of cancer in the Netherlands, after a notification of the PALGA ('Nationwide network and registry of histo- and cytopathology in the 
Netherlands') system. Afterwards, trained data collection registrars from the NCR extracted data from patients' records concerning patient characteristics, treatment and follow-up.

In this study, all patients diagnosed between 2005 and 2008 with primary invasive epithelial breast cancer and pN2a or pN3a statuses as the final pathologic result were included. Exclusion criteria were synchronous breast cancer, distant metastases at time of diagnosis (or within 91 days) or an unknown number of LNMs. Patients without surgery were also excluded. Data were collected on age, tumour type, receptor status, surgical procedures, systemic therapy, radiation therapy and pathological results, including pathologic TNM classification and the number of LNMs. For a period of 5 years after diagnosis, the first breast cancer event was registered, which consisted of any local, regional or contralateral recurrence or distant metastasis.

Patients with pN3a were divided into two subgroups according to the number of LNMs, to simulate pN3a based on infraclavicular or $\geq 10$ axillary LNMs. Patients with nine or less positive lymph nodes required at least one infraclavicular LNM to be considered pN3a, while patients with $\geq 10$ positive lymph nodes required at least 10 axillary LNMs (with or without infraclavicular LNMs).

\section{Treatment}

According to the national guideline of 2005, regional treatment depended on nodal status: sentinel lymph node biopsy (SLNB) in case of clinically node negative status, based on physical examination (axillary ultrasound was common but not mandatory at that time), or axillary lymph node dissection (ALND) in case of clinically node positive status, contraindication for SLNB or positive SLNB. ${ }^{10}$

Adjuvant irradiation of regional nodal fields was applied in case of four or more axillary LNMs or involvement of top axillary LNM. Recommended dose was 45-50 Gy in 5 weeks. Systemic therapy was generally recommended for all patients with LNM. Chemotherapy was advised in all premenopausal women and in women $<69$ years old with estrogen (ER) and progesterone (PR) tumours. In postmenopausal women, aged 50-59 years with ER+ PR+, chemotherapy was considered in physically fit patients and in women aged 60-69 years only if four or more nodes were involved. Chemotherapy regimen consisted of five courses 5 Fluorouracil, Epirubicin, Cyclophosphamide or six courses of Taxotere, Adriamycin and Cyclophosphamide. In case of Her2Neu receptor (HER 2) amplification, targeted therapy (Trastuzumab) was given in addition to chemotherapy. Endocrine therapy was recommended for all ER+ and/or PR+ tumours. 


\section{Statistics}

Statistical analyses were performed by using Statistical Package for the Social Sciences software (Version 22, IBM, Armonk, New York, USA). General characteristics between both subgroups were compared using chi-squared test for categorical data and MannWhitney U-test for continuous data.

For DFS, an event was defined as any local, regional or contralateral recurrence, distant metastasis or mortality within 5 years after the primary diagnosis. Events occurring 0-91 days after diagnosis were considered synchronous to the original tumour and not counted as recurrences. Date of death or date of emigration were derived from the Municipal Personal Records Database and completed until 31st December 2014. Patients were censored at the date of their first event, date of last follow-up, date of death or date of emigration, whatever came first.

DFS and OS for the pN3a subgroups, respectively, based on an infraclavicular LNM and $\geq 10$ axillary LNMs, were calculated with Kaplan-Meier curves and compared with the logrank test. ${ }^{11}$ P-Values (two-sided) $<0.05$ were considered statistically significant. Relevant clinicopathological variables associated with DFS and OS were examined using univariable and, where applicable, multivariable Cox proportional hazards regression, with hazard ratio (HR) and corresponding 95\% confidence intervals. The number of variables used for multivariable Cox proportional hazards regression depends on the number of outcome events per predictor variable, which requires at least five events per variable. $^{12}$

Finally, DFS and OS of patients with pN3a based on infraclavicular LNM were compared to patients with pN2a (i.e. 4-9 axillary LNMs), by calculating Kaplan-Meier curves and comparing with the log-rank test. In addition, univariable and multivariable Cox proportional hazards regression evaluated association of relevant clinicopathological variables associated with DFS and OS.

\section{Results}

A total of 51,239 patients were diagnosed with primary invasive epithelial breast cancer between 2005 and 2008 in the Netherlands, of whom 3442 patients had pN2a (6.6\%) and 1799 patients (3.5\%) had pN3a status (Figure 5.1). Eventually, 83 patients were classified as pN3a based on infraclavicular LNM (4.6\%) and 1705 patients based on $\geq 10$ axillary LNMs (95.4\%). Compared to patients with $\geq 10$ axillary LNMs, patients with at least an infraclavicular LNM were younger ( 55 versus 59 years, $p=0.010$ ), less often had pT3-4 tumours ( $15 \%$ versus $24 \%, p=0.049$ ) with a smaller mean tumour size (31 versus $36 \mathrm{~mm}, \mathrm{p}=0.032$ ) and, obviously, had fewer positive lymph nodes (mean 6 versus 15, 
$\mathrm{p}<0.001)$. A more detailed overview of the general characteristics is provided in Table 57.1.

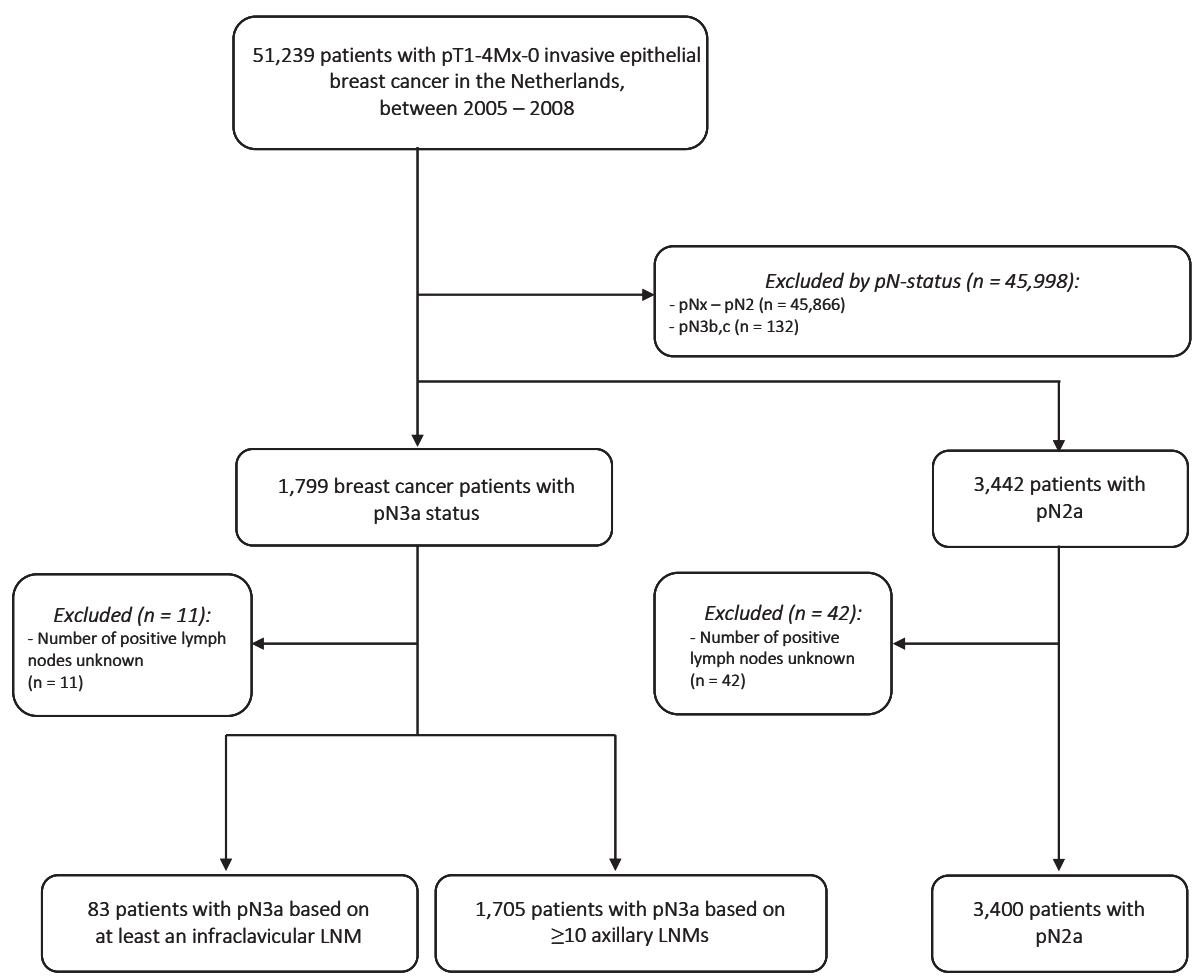

Figure 5.1 Flowchart of included patients. Abbreviations: $p N$ pathologic nodal status, LNM lymph node metastases.

Table 5.1 General patient and tumour characteristics.

\begin{tabular}{lccc}
\hline & $\begin{array}{c}\text { pN3a Infraclavicular LNM } \\
(\mathrm{n}=83)\end{array}$ & $\begin{array}{c}\text { pN3a } \geq 10 \text { axillary } \\
\text { LNMs } \\
(\mathrm{n}=1705)\end{array}$ & $\mathrm{p}$-value \\
\hline Mean age (years) (range) & $55.3(30-84)$ & $59.1(26-97)$ & 0.010 \\
Pathologic T-stage (\%) & $69(83.1)$ & $1252(73.4)$ & 0.049 \\
T0-2 & $12(14.5)$ & $406(23.8)$ & 0.049 \\
T3-4 & $2(2.4)$ & $47(2.8)$ & 0.850 \\
Unknown & $31.4(6-114)$ & $35.6(0-250)$ & 0.032 \\
Mean tumour size (mm) (range) & & & \\
Tumour grade (\%) & $39(47.0)$ & $700(41.1)$ & 0.284 \\
1-2 & $33(39.7)$ & $785(46.0)$ & 0.262 \\
3 & $11(13.3)$ & $220(12.9)$ & 0.926 \\
\hline Unknown & & & \\
\hline
\end{tabular}


Table $5.1 \quad$ (continued)

\begin{tabular}{|c|c|c|c|}
\hline & $\begin{array}{l}\text { pN3a Infraclavicular LNM } \\
\qquad(\mathrm{n}=83)\end{array}$ & $\begin{array}{c}\text { pN3a } \geq 10 \text { axillary } \\
\text { LNMs } \\
(n=1705)\end{array}$ & p-value \\
\hline Mean number of positive lymph nodes (range) & $5.7(1-9)$ & $15.2(10-53)$ & $<0.001$ \\
\hline \multicolumn{4}{|l|}{ ER (\%) } \\
\hline Positive & $64(77.1)$ & $1244(73.0)$ & 0.405 \\
\hline Negative & 19 (22.9) & $451(26.4)$ & 0.472 \\
\hline Unknown & 0 & $10(0.6)$ & 1.000 \\
\hline \multicolumn{4}{|l|}{ PR (\%) } \\
\hline Positive & $48(57.8)$ & $896(52.6)$ & 0.347 \\
\hline Negative & $34(41.0)$ & $723(42.4)$ & 0.795 \\
\hline Unknown & $1(1.2)$ & $86(5.0)$ & 0.183 \\
\hline \multicolumn{4}{|l|}{ Her2 (\%) } \\
\hline Positive & $12(14.5)$ & $346(20.3)$ & 0.195 \\
\hline Negative & $65(78.3)$ & $1247(73.1)$ & 0.298 \\
\hline Equivocal & $3(3.6)$ & $59(3.5)$ & 0.763 \\
\hline Unknown & $3(3.6)$ & $53(3.1)$ & 0.743 \\
\hline \multicolumn{4}{|l|}{ Tumour type (\%) } \\
\hline Invasive carcinoma NST & $58(69.9)$ & $1151(67.5)$ & 0.652 \\
\hline Lobular & $15(18.1)$ & $344(20.2)$ & 0.640 \\
\hline Mixed ductal and lobular & $5(6.0)$ & $92(5.4)$ & 0.805 \\
\hline Other & $5(6.0)$ & 118 (6.9) & 0.753 \\
\hline \multicolumn{4}{|l|}{ Subtype (\%) } \\
\hline $\mathrm{ER}+\mathrm{PR}+$, Her2- & $41(49.4)$ & $747(43.8)$ & 0.224 \\
\hline ER+PR-, Her2- & $13(15.7)$ & $241(14.1)$ & 0.697 \\
\hline $\mathrm{ER}+\mathrm{Her} 2+$ & $5(6.0)$ & $168(9.9)$ & 0.249 \\
\hline ER-Her2+ & $7(8.4)$ & $174(10.2)$ & 0.601 \\
\hline Triple negative & $12(14.5)$ & $238(14.0)$ & 0.898 \\
\hline Unknown & $4(4.8)$ & $127(7.4)$ & 0.369 \\
\hline \multicolumn{4}{|l|}{ Breast surgery (\%) } \\
\hline Breast conserving therapy & $22(26.5)$ & 339 (19.9) & 0.142 \\
\hline Mastectomy & $61(73.5)$ & 1363 (79.9) & 0.159 \\
\hline Unknown & 0 & $3(0.2)$ & 1.000 \\
\hline \multicolumn{4}{|l|}{ Axillary surgery (\%) } \\
\hline SLNB & $4(4.8)$ & $2(0.1)$ & $<0.001$ \\
\hline SLNB followed by ALND & $26(31.3)$ & $316(18.6)$ & 0.004 \\
\hline ALND & $53(63.9)$ & $1378(80.8)$ & $<0.001$ \\
\hline Unknown & 0 & $9(0.5)$ & 1.000 \\
\hline \multicolumn{4}{|l|}{ Radiation therapy (\%) } \\
\hline Yes & $76(91.6)$ & $1528(89.6)$ & 0.569 \\
\hline \multicolumn{4}{|l|}{ Chemotherapy (\%) } \\
\hline Yes & $67(80.7)$ & $1267(74.3)$ & 0.190 \\
\hline \multicolumn{4}{|l|}{ Endocrine therapy to ER+ subtype (\%) } \\
\hline Yes & $58(90.6)$ & $1130(90.8)$ & 0.955 \\
\hline \multicolumn{4}{|l|}{ Trastuzumab to Her2+ subtype (\%) } \\
\hline Yes & $8(66.7)$ & $254(73.4)$ & 0.604 \\
\hline
\end{tabular}

Abbreviations: ER estrogen, PR progesteron, Her2 human epidermal growth factor receptor 2, SLNB sentinel lymph node biopsy, ALND axillary lymph node dissection. 


\section{Disease-free survival}

Five year follow-up was available for 1293 patients $(72.3 \%, n=58$ versus $n=1235$, for patients with at least an infraclavicular versus $\geq 10$ axillary LNMs). Within 5 years after diagnosis, $43.6 \%$ experienced a first locoregional or contralateral recurrence or distance metastasis and $6.9 \%$ deceased. Thus $50.5 \%$ of the patients experienced an event, resulting in a DFS of $49.5 \%$. In subgroup analyses, DFS was $63.8 \%$ in patients with at least an infraclavicular LNM and $48.8 \%$ of patients with $\geq 10$ axillary LNMs $(p=0.018)$ (Figure 5.2a).

In multivariable Cox regression analyses, the effect of having $\geq 10$ axillary LNMs on DFS was significant (HR 1.59, $P=0.036$ ) (Table 5.2). Receiving chemotherapy (HR 0.51, $\mathrm{p}<0.001$ ) and radiation therapy (HR 0.59, $\mathrm{p}<0.001$ ) were identified as significant predictors for increased DFS, whereas triple negative subtype (HR 2.57, $p<0.001$ ) was identified as significant predictor for decreased DFS.

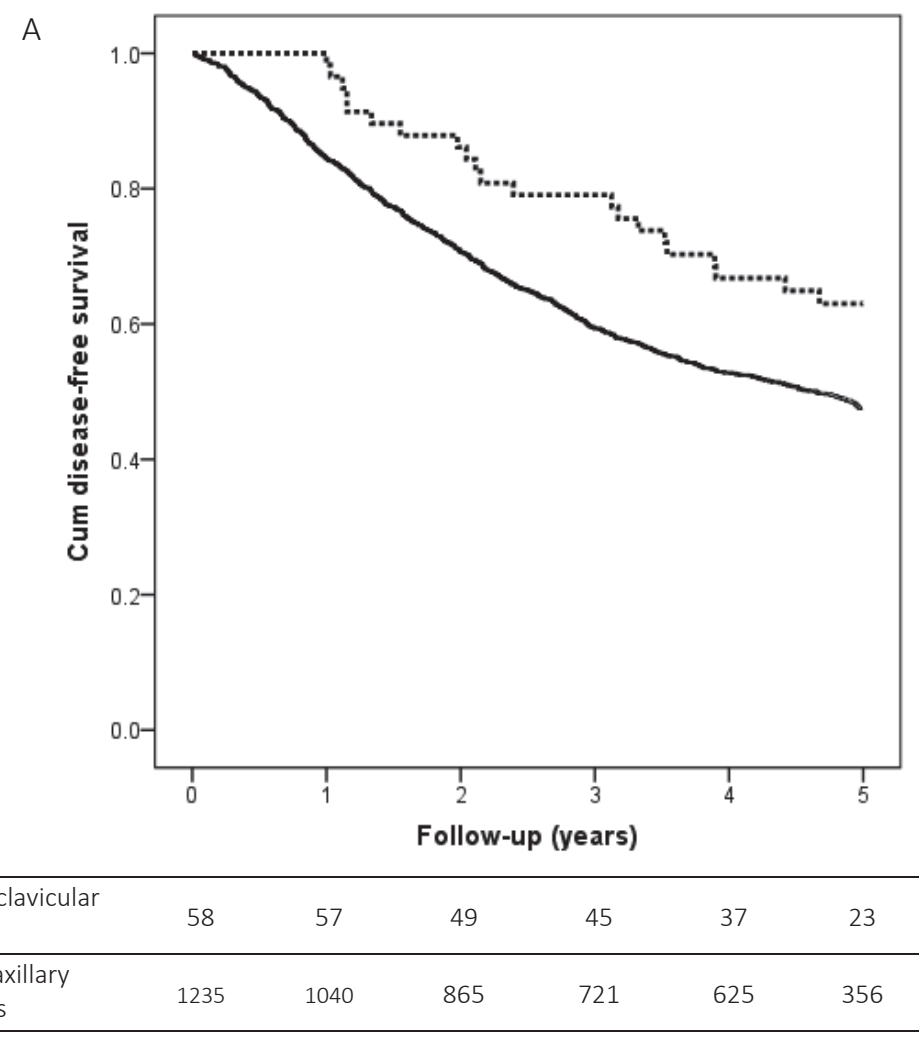


B

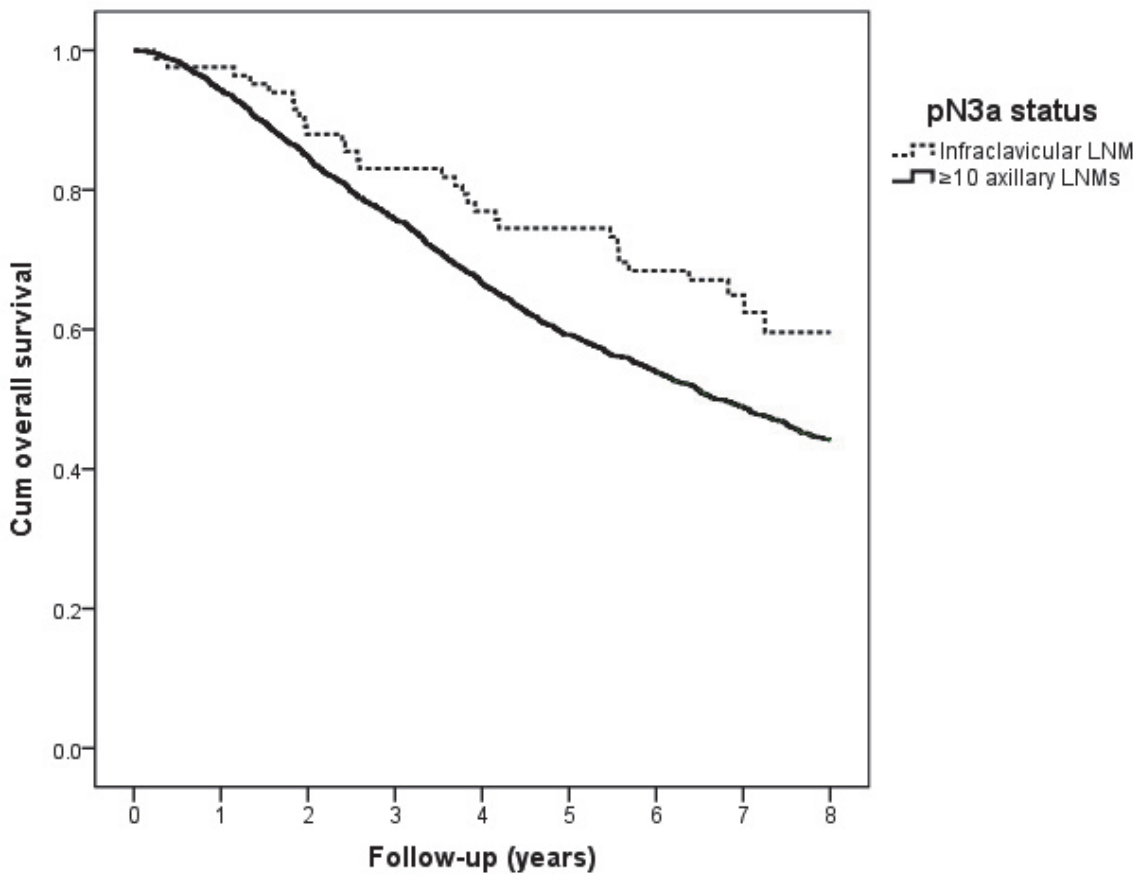

\begin{tabular}{lrrrrrrrrr}
\hline $\begin{array}{l}\text { Infraclavicular } \\
\text { LNM }\end{array}$ & 83 & 81 & 73 & 68 & 63 & 61 & 56 & 26 & 12 \\
\hline $\begin{array}{l}\geq 10 \text { axillary } \\
\text { LNMs }\end{array}$ & 1705 & 1607 & 1444 & 1288 & 1130 & 1004 & 912 & 627 & 373 \\
\hline
\end{tabular}

Figure 5.2 (A) and (B) Kaplan-Meier curves for disease-free survival after 5 years of follow up and overall survival after 8 years, including the number of patients at risk. LNM, lymph node metastases.

\section{Overall survival}

After 8 years of follow-up, $47.4 \%$ of all patients were alive. This concerned $63.9 \%$ of patients with at least an infraclavicular LNM and $46.6 \%$ with $\geq 10$ axillary LNMs ( $P=0.009)$ (Figure 5.2b).

In multivariable Cox regression analyses, the effect of having $\geq 10$ axillary LNMs on OS was statistically significant ( $H R$ 1.46, $p=0.042$ ) (Table 5.3). Significant predictors for decreased OS were the presence of pT3-4 tumours (HR 1.60, $p<0.001)$ and triple negative subtype ( $H R$ 1.79, $p<0.001)$. Receiving chemotherapy ( $H R$ 0.42, $p<0.001)$, endocrine therapy $(H R$ 0.60, $p<0.001)$ and radiation therapy $(H R 0.53, p<0.001)$ were identified as significant predictors for increased OS. 


\section{Comparison of infraclavicular LNM to pN2a nodal status}

In the subgroup of pN2a, 5-year follow-up was available for 2483 patients (73.0\%) with a DFS of $67.3 \%$. Compared to patients with pN3a based on infraclavicular LNM (DFS 63.8\%) this was not statistically significant ( $p=0.661$ ) (Appendix 5.1a). In multivariable Cox regression analyses, the effect of having pN3a based on infraclavicular LNM on DFS remained comparable to $\mathrm{pN2a}$ (HR 1.17, $\mathrm{p}=0.491$ ) (Appendix 5.2a).

After 8 years of follow-up, 65.5\% of pN2a patients were alive. Again, which was not different compared to OS of patients with pN3a based on infraclavicular LNM (OS 63.9\%) ( $p=0.500$ ) (Appendix 5.1b). In multivariable Cox regression analyses, the effect of having pN3a based on infraclavicular LNM on OS remained comparable to pN2a (HR 1.25, $\mathrm{p}=0.233$ ) Appendix 5.2b).

Table $5.2 \quad$ Uni- and multivariable analyses of predictors for disease-free survival.

\begin{tabular}{lcccc}
\hline & \multicolumn{2}{c}{ Univariable analysis } & \multicolumn{2}{c}{ Multivariable analysis } \\
\cline { 2 - 5 } & HR $(95 \% \mathrm{Cl})$ & $\mathrm{p}$-value & HR $(95 \% \mathrm{Cl})$ & $\mathrm{p}$-value \\
\hline Infraclavicular LNM & Reference & & Reference \\
$\geq 10$ axillary LNMs & $1.68(1.09-2.59)$ & 0.020 & $1.59(1.03-2.46)$ & 0.036 \\
Age (per year increment) & $1.02(1.02-1.03)$ & $<0.001$ & - & - \\
pT-stage T3-4 vs. T0-2 & $1.65(1.39-1.96)$ & $<0.001$ & - & - \\
Tumour grade 3 vs. 1-2 & $1.45(1.24-1.69)$ & $<0.001$ & - & $<0.001$ \\
Triple negative subtype Yes vs. No & $2.43(2.01-2.94)$ & $<0.001$ & $2.57(2.13-3.11)$ & $<0.001$ \\
Chemotherapy Yes vs. No & $0.49(0.42-0.58)$ & $<0.001$ & $0.51(0.43-0.60)$ & - \\
Trastuzumab Yes vs. No & $0.74(0.59-0.92)$ & 0.007 & - & - \\
Endocrine therapy Yes vs. No & $0.50(0.43-0.59)$ & $<0.001$ & $<0.001$ \\
Radiation therapy Yes vs. No & $0.48(0.37-0.60)$ & $<0.001$ & $0.59(0.46-0.75)$ & $<0.001$ \\
\hline
\end{tabular}

Abbreviations: LNM lymph node metastases, $p T$-stage pathologic tumour stage.

Table 5.3 Uni- and multivariable analyses of predictors for overall survival.

\begin{tabular}{lcccc}
\hline & \multicolumn{2}{c}{ Univariable analysis } & \multicolumn{2}{c}{ Multivariable analysis } \\
\cline { 2 - 5 } & HR $(95 \% \mathrm{Cl})$ & $\mathrm{p}$-value & HR $(95 \% \mathrm{Cl})$ & $\mathrm{p}$-value \\
\hline Infraclavicular LNM & Reference & & Reference \\
$\geq 10$ axillary LNMs & $1.61(1.12-2.32)$ & 0.010 & $1.46(1.01-2.10)$ & 0.042 \\
Age (per year increment) & $1.03(1.03-1.04)$ & $<0.001$ & - & - \\
pT-stage T3-4 vs T0-2 & $1.56(1.35-1.79)$ & $<0.001$ & $1.60(1.39-1.85)$ & $<0.001$ \\
Tumour grade 3 vs 1-2 & $1.43(1.26-1.63)$ & $<0.001$ & - & - \\
Triple negative subtype Yes vs No & $2.38(2.03-2.80)$ & $<0.001$ & $1.79(1.47-2.19)$ & $<0.001$ \\
Chemotherapy Yes vs No & $0.42(0.36-0.48)$ & $<0.001$ & $0.42(0.36-0.48)$ & $<0.001$ \\
Trastuzumab Yes vs No & $0.64(0.53-0.78)$ & $<0.001$ & - & - \\
Endocrine therapy Yes vs No & $0.51(0.45-0.58)$ & $<0.001$ & $0.60(0.51-0.71)$ & $<0.001$ \\
Radiation therapy Yes vs No & $0.38(0.32-0.45)$ & $<0.001$ & $0.53(0.44-0.64)$ & $<0.001$ \\
\hline
\end{tabular}

Abbreviations: LNM lymph node metastases, $p$ T-stage pathologic tumour stage. 


\section{Discussion}

According to the sixth and seventh edition of the TNM classification for breast cancer, pathologic nodal status is defined using the number and location of LNMs. A pN3a status in breast cancer consists either of at least an infraclavicular (level III) or $\geq 10$ axillary LNMs. ${ }^{7}$ Inclusion of both groups in the same category of TNM suggests a similar prognosis. ${ }^{8,9,13}$ However, our study demonstrated superior DFS and OS in patients with pN3a based on at least an infraclavicular LNM compared to $\geq 10$ axillary LNMs. Furthermore, DFS and OS of patients with pN3a based on at least an infraclavicular LNM compared to patients with pN2a were comparable according to our study.

The decision to redefine infraclavicular LNM as pN3a breast cancer was solely based on the results of a study of Newman et al.. ${ }^{2,6}$ In this study, incidence and prognosis of infraclavicular LNM among patients with axillary LNMs was investigated, which showed worse DFS and OS in case of suspicious adenopathy. ${ }^{6}$ Study limitations were the absence of pathological confirmation of the suspicious nodes, as well as potentially confounding factors like presence of supraclavicular LNM in some patients. ${ }^{14}$ In our study, pN3a was defined according to final pathological report, resulting in pathologically confirmed infraclavicular LNM in all 83 patients and therefore representing a more valid patient population.

Classification of infraclavicular LNM as pN3a disregards the number of nodal metastases and the size of the largest metastasis. A single micrometastasis in an infraclavicular lymph node would represent pN3a status, whereas pN3a without infraclavicular involvement would require $\geq 10$ LNMs with at least one macrometastasis. ${ }^{7}$ Disregarding size of the nodal metastases by only taking infraclavicular location into account can explain part of the difference in DFS and OS between pN3a based on at least an infraclavicular LNM and $\geq 10$ axillary LNMs.

DFS after 5 years in patients with $\geq 10$ axillary LNMs in our study cohort is comparable to previous results of Koca et al., in which 5 -year DFS was $46.2 \%$ in patients with $\geq 10$ axillary LNMs. ${ }^{15}$ In a similar cohort of patients with $\geq 10$ axillary LNMs, Turker et al. demonstrated the highest 5-year DFS rate of $49.2 \%$ in patients with ER/PRp and Her2subtype. ${ }^{16}$ These results confirm our findings concerning DFS in patients with pN3a based on $\geq 10$ axillary LNMs, which was $48.8 \%$.

The definition of an infraclavicular (level III) lymph node during surgery may be open to interpretation. According to the American Society of Breast Surgeons, a level lelll ALND (extending to the apex of the axilla) is only recommended in patients with evidence of suspicious nodes located behind the pectoralis minor muscle (level II). ${ }^{17}$ However, in some cases a suspicious level II node can be incorrectly defined as infraclavicular (level III) node during ALND. As a consequence, these patients were considered pN3a based on 
infraclavicular LNM rather than potentially pN2a or pN1a, depending on the total number of axillary LNMs.

Due to new imaging techniques, the detection of infraclavicular LNM has increased over time. Prior to the introduction of the sixth edition of TNM in 2002, infraclavicular LNMs were detected during physical examination and/or surgery. In the current era, with imaging modalities like breast magnetic resonance imaging (MRI) and positron-emission tomography/computed tomography (PET/CT), smaller (infraclavicular) LNMs can be detected prior to surgery. ${ }^{18,19}$ However, the seventh edition of TNM is still based on a 2001 study in which infraclavicular LNMs were detected with physical examination and ultrasound rather than MRI or PET-CT. ${ }^{2,6}$ Our cohort consisted of patients diagnosed between 2005 and 2008, which is more in line with the current imaging modalities. MRI was already recommended in our study cohort according to the national guidelines of 2005. ${ }^{7,10}$

Although the incidence of patients with pN3a based on at least an infraclavicular LNM in our cohort is small (4.6\%), our findings suggest that reclassification in the next TNM classification should be considered. We advise to redefine an infraclavicular LNM as equivalent to other axillary LNMs rather than taking the location of infraclavicular LNM into account. Consequently, patients with an infraclavicular LNM with $\leq 9$ LNMs will be considered pN2a rather than pN3a. In this way, infraclavicular LNM will become consistent with intramammary and interpectoral LNM, which are coded as axillary LNMs (level $\mathrm{I} / \mathrm{II}$ ) in the current TNM classification. ${ }^{7}$ Yet, adjuvant (radiation) therapy of infraclavicular LNM is still recommended.

This study had limitations. A major limitation of this study concerns the subgroup of patients with $\geq 10$ axillary LNMs, which still might have infraclavicular LNM as well. Yet, the focus of this study was to compare prognosis between both subgroups, since the current TNM atlas considers both as one category. Our results should therefore be interpreted with this important limitation in mind.

A second limitation of this study was the use of a retrospective database. Some clinically relevant parameters were not present, for instance, radiation therapy fields and the presence of lymphovascular invasion of the tumour. As a consequence, irradiation of regional nodal fields is unknown in this study cohort, which is generally recommended in breast cancer patients with LNMs. ${ }^{20}$ Furthermore, presence of lymphovascular invasion can have a negative effect on overall survival. ${ }^{21}$

Third, these data only contain patients treated in the Netherlands between 2005 and 2008. This might have influence on prognosis when these data would be extrapolated to cohorts in other countries. For instance, the 5-year survival rate of breast cancer patients in the Netherlands still is different when compared to Asian or South American countries. $^{22}$ 
Fourth, despite the collection of nationwide data between 2005 and 2008 in the Netherlands, the subgroup of patients with pN3a based on at least an infraclavicular LNM remained small ( $n=83)$. As a consequence, the number of variables for multivariable Cox proportional hazards regression was restricted due to the limited number of events. ${ }^{12}$ However, differences between both subgroups regarding tumour subtypes and adjuvant treatment were small, which means that it is unlikely that the difference in prognosis would be attributable to the difference in covariates between the two cohorts.

In conclusion, DFS and OS of patients staged as pN3a based on at least an infraclavicular LNM is superior compared to patients with $\geq 10$ axillary LNMs. Therefore, reclassification of infraclavicular LNM in the next edition of TNM should be considered to classify an infraclavicular LNM with fewer than 10 LNMs to pN2a rather than pN3a. 


\section{References}

1. UICC, Committee on Clinical Stage Classification and Applied Statistics. Clinical stage classification and presentation of results, malignant tumors of the breast and larynx. Paris: International Union Against Cancer; 1958.

2. Singletary SE, Greene FL, Breast Task F. Revision of breast cancer staging: the 6th edition of the TNM classification. Semin Surg Oncol 2003;21:53-59.

3. Sobin LH, Fleming ID. TNM classification of malignant tumors, fifth edition (1997). Union Internationale Contre le Cancer and the American Joint Committee on Cancer. Cancer 1997;80:1803-1804.

4. Beenken SW, Urist MM, Zhang Y, Desmond R, Krontiras H, Medina H, et al. Axillary lymph node status, but not tumor size, predicts locoregional recurrence and overall survival after mastectomy for breast cancer. Ann Surg 2003;237:732-8. discussion 738-739.

5. Sobin L, Wittekind C. International union against cancer (UICC): TNM classification of malignant tumors 6th edition. New York: WilleyeLiss; 2002.

6. Newman LA, Kuerer HM, Fornage B, Mirza N, Hunt KK, Ross MI, et al. Adverse prognostic significance of infraclavicular lymph nodes detected by ultrasonography in patients with locally advanced breast cancer. Am J Surg 2001;181:313-318.

7. Sobin LH, Gospodarowicz MK, Wittekind C. TNM classification of malignant tumours. John Wiley \& Sons; 2011.

8. Gospodarowicz M, Mackillop W, O'Sullivan B, Sobin L, Henson D, Hutter RV, et al. Prognostic factors in clinical decision making: the future. Cancer 2001;91(8 Suppl.):1688-1695.

9. Webber C, Gospodarowicz M, Sobin LH, Wittekind C, Greene FL, Mason MD, et al. Improving the TNM classification: findings from a 10-year continuous literature review. Int J Cancer 2014;135:371-378.

10. СBO Kwaliteitsinstituut voor de Gezondheidszorg. Vereniging van Integrale Kankercentra. Guideline 'Treatment of breast cancer' (Richtlijn 'Behandeling van het Mammacarcinoom'). 2005.

11. Kaplan EL, Meier P. Nonparametric estimation from incomplete observations. J Am Stat Assoc 1958;53:457-481.

12. Vittinghoff $\mathrm{E}, \mathrm{McC}$ (lloch $\mathrm{CE}$. Relaxing the rule of ten events per variable in logistic and Cox regression. Am J Epidemiol 2007;165:710-718.

13. Veronesi U, Viale G, Rotmensz N, Goldhirsch A. Rethinking TNM: breast cancer TNM classification for treatment decisionmaking and research. Breast 2006;15:3-8.

14. Brito RA, Valero V, Buzdar AU, Booser DJ, Ames F, Strom E, et al. Long-term results of combined-modality therapy for locally advanced breast cancer with ipsilateral supraclavicular metastases: the University of Texas M.D. Anderson Cancer Center experience. J Clin Oncol 2001;19:628-633.

15. Koca E, Kuzan TY, Dizdar O, Babacan T, Sahin I, Ararat E, et al. Outcomes of locally advanced breast cancer patients with $\geq 10$ positive axillary lymph nodes. Med Oncol 2013;30:615.

16. Turker I, Arslan UY, Yazici O, Uyeturk U, Oksuzoglu B, Budakoglu B, et al. Prognostic factors in operated stage IIIC, pathological N3a breast cancer patients. Breast care (Basel, Switzerland) 2014;9:421-427.

17. American Society of Breast Surgeons. Performance and Practice guidelines for axillary lymph node dissection in breast cancer patients. Available from: https://www.breastsurgeons.org/ new_layout/about/statements/PDF_Statements/PerformancePracticeGuidelines_ALND.pdf. Visited: 176-2016.

18. Kuijs VJ, Moossdorff M, Schipper RJ, Beets-Tan RG, Heuts EM, Keymeulen KB, et al. The role of MRI in axillary lymph node imaging in breast cancer patients: a systematic review. Insights Imaging 2015;6: 203-215.

19. Koolen BB, Valdes Olmos RA, Vogel WV, Vrancken Peeters MJ, Rodenhuis S, Rutgers EJ, et al. Prechemotherapy 18F-FDG PET/CT upstages nodal stage in stage II-III breast cancer patients treated with neoadjuvant chemotherapy. Breast Cancer Res Treat 2013;141:249-254.

20. Senkus E, Kyriakides S, Ohno S, Penault-Llorca F, Poortmans P, Rutgers E, et al. Primary breast cancer: ESMO clinical practice guidelines for diagnosis, treatment and follow-up. Ann Oncol 2015;26(Suppl. 5):v8-30.

21. Houvenaeghel G, Classe JM, Garbay JR, Giard S, Cohen M, Faure C, et al. Survival impact and predictive factors of axillary recurrence after sentinel biopsy. Eur J Cancer 2016;58:73-82. 
22. Allemani C, Weir HK, Carreira H, Harewood R, Spika D, Wang XS, et al. Global surveillance of cancer survival 1995e2009: analysis of individual data for 25,676,887 patients from 279 population-based registries in 67 countries (CONCORD-2). Lancet 2015;385977-1010. 
Appendix 7.1

A

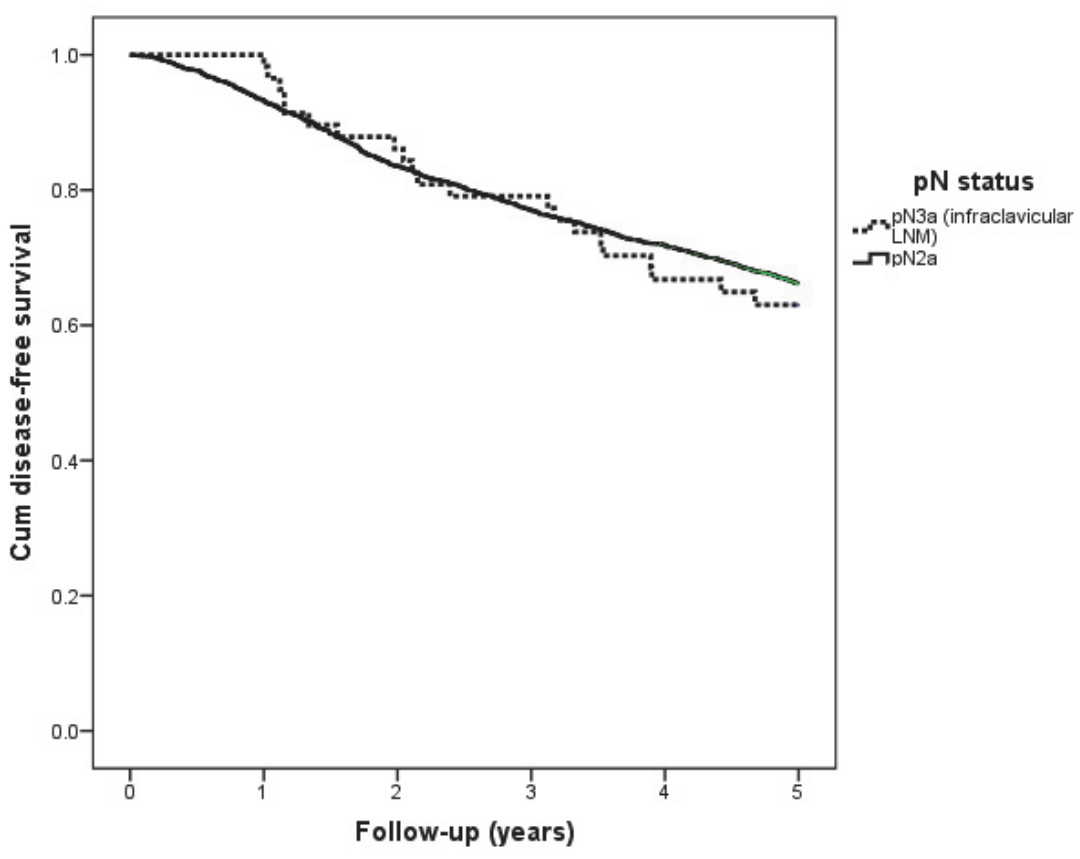

\begin{tabular}{lrrrrrr}
\hline $\begin{array}{l}\text { Infraclavicular } \\
\text { LNM }\end{array}$ & 58 & 57 & 49 & 45 & 37 & 23 \\
\hline pN2a & 2483 & 2317 & 2076 & 1916 & 1791 & 1670 \\
\hline
\end{tabular}


B

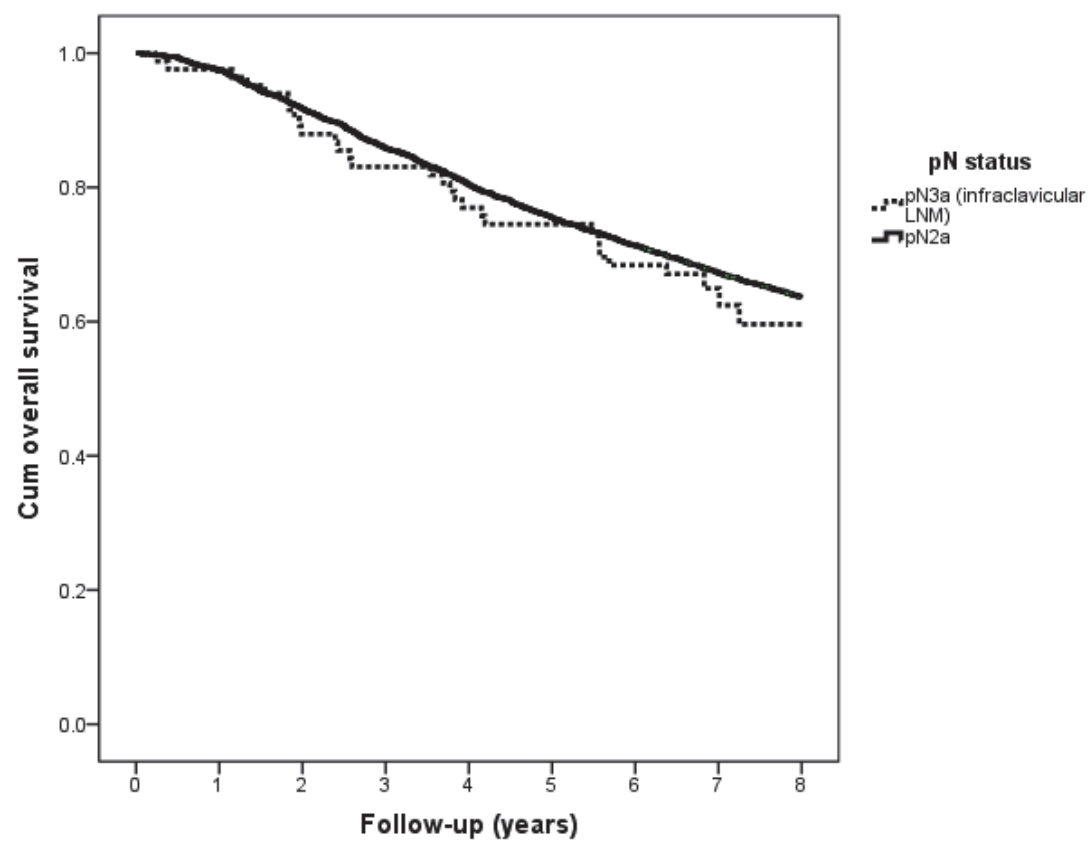

\begin{tabular}{lrrrrrrrrr}
\hline $\begin{array}{l}\text { Infraclavicular } \\
\text { LNM }\end{array}$ & 83 & 81 & 73 & 68 & 63 & 61 & 56 & 26 & 12 \\
\hline $\begin{array}{l}\geq 10 \text { axillary } \\
\text { LNMs }\end{array}$ & 1705 & 1607 & 1444 & 1288 & 1130 & 1004 & 912 & 627 & 373 \\
\hline
\end{tabular}




\section{Appendix 7.2}

A

\begin{tabular}{lcccc}
\hline & \multicolumn{2}{c}{ Univariable analysis } & \multicolumn{2}{c}{ Multivariable analysis } \\
\cline { 2 - 5 } & \multicolumn{1}{c}{ HR $(95 \% \mathrm{Cl})$} & P-value & HR $(95 \% \mathrm{Cl})$ & $P$-value \\
\hline pN2a & Reference & & Reference & 0.491 \\
\hline Infraclavicular LNM & $1.10(0.71-1.70)$ & 0.661 & $1.17(0.76-1.80)$ & - \\
\hline Age (per year increment) & $1.03(1.02-1.03)$ & $<0.001$ & - & - \\
pT-stage T3-4 vs T0-2 & $1.94(1.64-2.31)$ & $<0.001$ & - & - \\
\hline Tumour grade 3 vs 1-2 & $1.65(1.44-1.88)$ & $<0.001$ & $<0.001$ \\
Triple negative subtype Yes vs No & $2.92(2.47-3.46)$ & $<0.001$ & $3.08(2.60-3.65)$ & $<0.001$ \\
\hline Chemotherapy Yes vs No & $0.41(0.35-0.47)$ & $<0.001$ & $0.44(0.38-0.51)$ & - \\
Trastuzumab Yes vs No & $0.72(0.59-0.88)$ & 0.001 & - & - \\
\hline Endocrine therapy Yes vs No & $0.42(0.36-0.48)$ & $<0.001$ & - & $<0.001$ \\
Radiation therapy Yes vs No & $0.40(0.33-0.49)$ & $<0.001$ & $0.52(0.43-0.64)$ & - \\
\hline
\end{tabular}

Abbreviations: LNM lymph node metastases, $p T$-stage pathologic tumour stage.

B

\begin{tabular}{lcccc}
\hline & \multicolumn{2}{c}{ Univariable analysis } & \multicolumn{2}{c}{ Multivariable analysis } \\
\cline { 2 - 5 } & HR $(95 \% \mathrm{Cl})$ & $P$-value & HR $(95 \% \mathrm{Cl})$ & $P$-value \\
\hline pN2a & Reference & & Reference & \\
Infraclavicular LNM & $1.13(0.79-1.63)$ & 0.501 & $1.25(0.87-1.79)$ & 0.233 \\
\hline Age (per year increment) & $1.04(1.04-1.05)$ & $<0.001$ & - & - \\
pT-stage T3-4 vs T0-2 & $1.88(1.64-2.18)$ & $<0.001$ & $1.82(1.58-2.10)$ & $<0.001$ \\
\hline Tumour grade 3 vs 1-2 & $1.50(1.34-1.68)$ & $<0.001$ & - & - \\
Triple negative subtype Yes vs No & $2.68(2.32-3.10)$ & $<0.001$ & $1.75(1.47-2.10)$ & $<0.001$ \\
\hline Chemotherapy Yes vs No & $0.31(0.28-0.35)$ & $<0.001$ & $0.34(0.30-0.38)$ & $<0.001$ \\
Trastuzumab Yes vs No & $0.62(0.52-0.74)$ & $<0.001$ & - & - \\
\hline Endocrine therapy Yes vs No & $0.44(0.39-0.49)$ & $<0.001$ & $0.51(0.45-0.59)$ & $<0.001$ \\
Radiation therapy Yes vs No & $0.34(0.30-0.40)$ & $<0.001$ & $0.56(0.48-0.65)$ & $<0.001$ \\
\hline
\end{tabular}

Abbreviations: LNM lymph node metastases, $p T$-stage pathologic tumour stage. 
III

\section{Part 2 - Individual risk \& timing of recurrences during follow-up}





\section{Chapter 6}

Local recurrence after mastectomy for breast cancer in the current era: which subgroups are still at risk?

Moossdorff M, Smit L, van Nijnatten T, van Kuijk S, Keymeulen KBMI, de Boer M, Boersma L, Strobbe LJA, Siesling S, Smidt ML

Submitted 


\section{Abstract}

\section{Background}

The incidence of local recurrence (LR) after mastectomy has decreased. However, preventing LR is still a major goal of local treatment. The indication for post-mastectomy radiation therapy is based on traditional risk factors. Recently, emphasis has shifted from traditional risk factors (e.g. T-stage, nodal involvement) to tumor biology, (e.g. receptor status, molecular diagnostics). The risk of LR might vary between breast cancer subtypes. The aim of this study was to determine the risk of LR as a first event after mastectomy for breast cancer subtypes in the current era.

\section{Methods}

From the Netherlands Cancer Registry, including data from all hospitals in the Netherlands, all new invasive epithelial breast cancers (MO) treated with mastectomy, diagnosed in 2005-2008 were included. Endpoints were incidence of and predictors for LR after mastectomy as a first event within 5 years, overall and in subtypes of breast cancer.

\section{Results}

In total, 15382 breast cancers were analyzed, which were treated with radiotherapy in $29.8 \%$, chemotherapy in $45.9 \%$, endocrine therapy in $69 \%$ of ER+ and trastuzumab (in Her2+ tumors) in 58.3\%. Overall, 5-year LR as a first event occurred in 3.8\%. This was 2.8\% in ER+PR+Her2-, 3.1\% in ER+PR-Her2-, 3.0\% in ER+Her2+, $4.7 \%$ in ER-Her2+, and $9.5 \%$ in triple negative tumors. ER+HER2+ and ER-Her2+ cancers that were treated with both trastuzumab and chemotherapy had significantly fewer LR compared to treatment with chemotherapy alone (2.0\% vs 6.0\% in ER+Her2+ and 3.5\% vs 6.9\% in ER-Her2+). The strongest independent predictors of $L R$ in the overall population were endocrine therapy (protective, versus no endocrine therapy, HR 0.29[95\% Cl 0.23-0.36]), >3 positive nodes (versus 1-3, HR 2.29[1.63-3.21]) and T4-stage (versus T0-1, HR 5.50[3.05-8.38]). The strength of the predictors varied between subtypes, particularly for the number of positive nodes, radiation therapy, and T-stage.

\section{Conclusion}

Currently, particularly triple negative tumors are at risk for LR after mastectomy. Commonly known risk factors (number of positive nodes, T4-stage, and no endocrine therapy) were confirmed, but their importance varied between subtypes. Local treatment should be tailored to breast cancer subtype and trials investigating local treatment should report results stratified on different breast cancer subtypes. 


\section{Introduction}

The incidence of local recurrence (LR) after mastectomy has decreased over the last decades, resulting from better diagnostics, surgery, radiotherapy, and systemic treatment, such as anthracycline and taxane chemotherapy and trastuzumab. Preventing LR remains a major goal of local treatment.

An estimated high risk of LR prompts recommending post-mastectomy radiation therapy (PMRT). This estimate is based on traditional risk factors, such as nodal stage, tumor stage, lymphovascular invasion (LVI), tumor grade, and age. ${ }^{1-3}$ The recommendations based on nodal stage are widely used and based mainly on the EBCTCG meta-analyses. First, the indication was established for high risk patients, i.e. with $>3$ positive nodes or >T3 tumors, and later also for intermediate risk patients (1-3 involved nodes, or T2 tumors with LVI or grade 3). ${ }^{4,5}$ However, locoregional recurrence (LRR) rate in the included trials was $20-30 \%$, which is much higher than observed recently. ${ }^{6}$ Additionally, the studies in the EBCTCG meta-analyses enrolled between 1961 and 1988. Therefore, they reflect a different population with more unfavorable characteristics, resulting from absence of screening and no or suboptimal systemic therapy compared to nowadays (CMF instead of antracyclines and taxanes; no trastuzumab). Also, radiation techniques and planning have improved (e.g., 3D instead of 2D techniques). Finally, radiation fields varied between the trials and were generally more extensive (including the axilla, supraclavicular fossa and internal mammary chain) than many clinics would currently use. These differences in incidence and patient management may all impact the risk of LR. As a consequence, risk assessment, and the potential benefit derived from these trials may not be applicable in the current era.

Furthermore, emphasis in breast cancer research has shifted from traditional risk factors (e.g. T-stage and N-stage) to a tumor biology based approach (intrinsic subtypes, molecular profiling). It is conceivable that different subtypes of breast cancer pose different risks for LR after mastectomy, and different absolute and/or relative benefit from radiation therapy. Several studies have addressed risk of LR after mastectomy in subtypes of breast cancer, some also including Her2 status. ${ }^{7-9}$ However, these studied older cohorts, that were often treated without modern systemic therapy and trastuzumab. A nomogram to assess LRR risk was also proposed (although not specific for mastectomy and type of surgery was not significant on univariable analysis), but Her2 status was not known for this population. ${ }^{10}$ Thus, studies assessing LR risk for different subtypes of breast cancer, treated in the current era, including Her2-status and treatment with trastuzumab, are lacking. 
This study aims to determine 5-year risk of LR as a first event after mastectomy in different breast cancer subtypes, treated in the current era. Additionally, it aims to determine factors that predict 5-year LR in different subtypes. If absolute risk and risk factors for LR differ per subtype, local therapy should be tailored to subtype and trials investigating local therapy should report results separately for different subtypes.

\section{Methods}

\section{Data collection}

The Netherlands Cancer Registry (NCR) contains data on all new cancer patients in The Netherlands. Trained data managers of the Comprehensive Cancer Organisation the Netherlands (IKNL) gather data from patients' records. The database includes patient and tumor characteristics, as well as surgical, radiation, and systemic treatment. For a period of 5 years after diagnosis, the first breast cancer event was registered (LR, contralateral breast cancer, regional recurrence, or distant recurrence).

\section{Included patients}

From this database, all new epithelial breast cancers in women diagnosed between 2005-2008 and treated with mastectomy were included. Patients with distant metastasis at diagnosis (or within 91 days) were excluded.

\section{Treatment according to the national guideline}

According to the guideline of $2005^{11}$ (in effect at the time of diagnosis for this cohort), regional treatment consisted of sentinel lymph node biopsy (SLNB) in clinically node negative breast cancer, based on physical examination (axillary ultrasound was common but not mandatory). Contraindications for SLNB were $>T 2$, multiple tumors, and previous axillary surgery. If positive nodes were identified preoperatively, or SLNB was contraindicated, or SLNB was positive, axillary lymph node dissection (ALND) was performed. Chest wall irradiation was recommended for positive margins, T4 tumors, involvement of the pectoralis muscle, and was considered individually for pT3 tumors. Chest wall irradiation including regional nodal fields was applied in case of $\geq p N 2$ or involvement of upper medial axillary nodes. Recommended dose was 45-50Gy in 5 weeks, and boost to 60-70Gy in case of residual tumor.

Indication for systemic treatment depended on nodal involvement, age, tumor size, grade, and receptor status. In N+ breast cancer, endocrine therapy was recommended for all ER+ and/or PR+ tumors. Chemotherapy was advised for $\mathrm{N}+$ breast cancer in all 
premenopausal women, and in women $<69$ years with ER- and PR- tumors. In postmenopausal women aged 50-59 with ER+PR+ and $\mathrm{N}+$ tumors, chemotherapy was considered in fit patients, and in women aged 60-69 only if 4 or more of nodes were involved.

For NO breast cancer, systemic therapy (chemotherapy and endocrine therapy for ER+ or PR+ tumors and chemotherapy for ER-PR-tumors) was considered for patients $\leq 35$ years (except grade I tumors $\leq 1 \mathrm{~cm}$ ), and patients $>35$ years with tumors $\geq 3 \mathrm{~cm}$, or $\geq 1 \mathrm{~cm}$ and grade III, or $\geq 2 \mathrm{~cm}$ and grade $\mathrm{II}$.

Chemotherapy consisted of 5 courses of FEC or 6 courses of TAC. If chemotherapy was indicated for a Her2+ tumor, patients were treated with trastuzumab. Endocrine therapy consisted of 2-3 years of tamoxifen and aromatase inhibitor for 3-2 years, or 5 years of aromatase inhibitor for postmenopausal women, or 5 years of tamoxifen for premenopausal women, optionally including LHRH agonist if not postmenopausal after chemotherapy.

\section{Pathology \& subtypes}

Five different subtypes of breast cancer were studied, namely ER+PR+Her2-, ER+PRHer2-, ER+Her2+, ER-Her2+, and triple negative tumors. Tumors were considered ER+ and $\mathrm{PR}+$ if more than $10 \%$ of tumor cells showed nuclear staining on immunohistochemistry (IHC). Her2 status was evaluated with at least IHC, in which 3+ was considered positive ( $>10 \%$ of cells show circumferential membrane staining with strong intensity) and 0 and $1+$ negative $(<10 \%$ circumferential membrane staining, or $>10 \%$ membrane staining but weak intensity). In case of $2+$ on IHC ( $>10 \%$ circumferential membrane staining with moderate intensity), the guideline advised FISH for confirmation. The result of FISH overruled the result of IHC. If subtype could not be determined, the case was disregarded for all subtype analyses.

\section{Endpoints}

The primary endpoint was LR as a first event within 5 years after diagnosis. LR was defined as any invasive breast cancer on the ipsilateral thoracic wall including the mastectomy scar, i.e. both LR and new primary ipsilateral breast cancer were counted. ${ }^{12}$ Events occurring 0-91 days after diagnosis were regarded synchronous to the original tumor and not counted as recurrences. Patients were censored at the date of their first event, at the date of last follow up, or at the date of death. If another event occurred within 91 days of the first recurrence, this was considered synchronous to the first event, and also counted as a first recurrence. 


\section{Statistical analysis}

Analyses were performed using SPSS [IBM Corporation, version 22/23.0.0.0] and R [R foundation, version 3.3.2]. LR incidence was determined for the whole cohort and the subtypes using Kaplan-Meier analysis. Significance of the difference between the subtypes was tested with the Log-rank test. Univariable and multivariable Cox regression models were used to determine risk factors for LR, overall and in subtypes. Factors that likely influence the probability of LR after mastectomy were included in the multivariable analysis. The proportional hazards assumption was tested by visual inspection of logminus-log plots. In case of doubt, scaled Schoenfeld residuals were calculated and the proportional hazards assumption was tested by assessing the correlation of the Schoenfeld residuals with time. A slope different from zero indicates a violation of the proportional hazards assumption. P-values of $<0.05$ were considered statistically significant.

\section{Results}

In total 15382 new epithelial invasive breast cancers, diagnosed between 2005 and 2008 and treated with mastectomy were analyzed. Baseline characteristics are shown in $3.5 \%$ equivocal). Subtype could not be determined in $13.7 \% \quad(n=2106)$. PRMT was administered in $29.8 \%$, chemotherapy in $45.9 \%$, endocrine therapy to $69.1 \%$ of ER+ tumors, and trastuzumab to $58.3 \%$ of Her2+ tumors. Adjuvant treatment per subtype is shown in Table S6.1.

\section{Incidence of $L R$ after mastectomy}

Median follow-up time was 57.7 months. LR after mastectomy as a first event occurred in 3.8\% (Table 6.2/Figure 6.1). The risk of LR varied between subtypes, and was lowest for ER+PR+Her2- tumors (2.8\%) and highest for triple negative tumors (9.5\%). The overall difference between the subtypes was statistically significant (Log Rank (MantelCox) test, Chi-square $(4)=166.039, \mathrm{p}<0.001)$. Univariable Cox regression was used to compare subtypes to the most favorable subtype. Compared to ER+PR+Her2-, no significant difference existed for ER+PR-Her2- tumors (HR 1.155 [95\% Cl 0.839-1.589], $p=0.377)$ and $E R+H e r 2+(H R$ 1.096 [0.766-1.569], $p=0.616)$, in ER-Her2+ and triple negative breast cancers significantly more LR occurred. 
Table 6.1 Baseline characteristics

\begin{tabular}{|c|c|c|c|c|c|}
\hline & & $\mathrm{N}(\%)$ & & & $\mathrm{N}(\%)$ \\
\hline $\begin{array}{l}\text { Median age } \\
\text { (range) }\end{array}$ & & $59.0(20-100)$ & Morphology & Ductal & $10750(69.9 \%)$ \\
\hline \multirow[t]{6}{*}{ pT-stage } & TO & $173(1.1 \%)$ & & Lobular & 2233 (14.5\%) \\
\hline & $\mathrm{T} 1$ & $6641(43.2 \%)$ & & $\begin{array}{l}\text { Mixed ductal \& } \\
\text { lobular }\end{array}$ & 753 (4.9\%) \\
\hline & $\mathrm{T} 2$ & 6866 (44.6\%) & & Other & $1647(10.7 \%)$ \\
\hline & T3 & 992 (6.4\%) & Residual tumor & No & $14542(94.5 \%)$ \\
\hline & $\mathrm{T} 4$ & $312(2.0 \%)$ & & Microscopic & $559(3.6 \%)$ \\
\hline & Tx & $398(2.6 \%)$ & & Macroscopic & $33(0.2 \%)$ \\
\hline \multirow[t]{6}{*}{ pN-stage } & NO & 7433 (48.3\%) & & Unknown & $248(1.6 \%)$ \\
\hline & N1mi & $861(5.6 \%)$ & & & \\
\hline & N1 & $3976(25.8 \%)$ & Radiation & Yes & 4581 (29.8\%) \\
\hline & N2 & 1799 (11.7\%) & therapy & No & $10801(70.2 \%)$ \\
\hline & N3 & $1102(7.2 \%)$ & Chemotherapy & Yes & 7057 (45.9\%) \\
\hline & $\mathrm{Nx}$ & $211(1.4 \%)$ & & No & 8325 (54.1\%) \\
\hline \multirow[t]{4}{*}{ Grade } & 1 & 2449 (15.9\%) & & Neoadjuvant & $1343(8.7 \%)$ \\
\hline & 2 & 6275 (40.8\%) & Endocrine & Yes & $8256 / 11948(69.1 \%)$ \\
\hline & 3 & $5051(32.8 \%)$ & $\begin{array}{l}\text { therapy for ER+ } \\
\text { tumors }\end{array}$ & No & $3692 / 11948$ (30.9\%) \\
\hline & Unknown & 1607 (10.4\%) & Trastuzumab for & Yes & $1509 / 2589(58.3 \%)$ \\
\hline \multirow[t]{3}{*}{ ER } & Positive & $11948(77.7 \%)$ & Her2+ tumors & No & $1080 / 2589$ (41.7\%) \\
\hline & Negative & 3208 (20.9\%) & & & \\
\hline & Unknown & $226(1.5 \%)$ & Subtype & $\mathrm{ER}+\mathrm{PR}+\mathrm{Her} 2-$ & 7296 (47.4\%) \\
\hline \multirow[t]{3}{*}{ PR } & Positive & 9182 (59.7\%) & & ER+PR-Her2- & $1822(11.8 \%)$ \\
\hline & Negative & $5383(35.0 \%)$ & & $\mathrm{ER}+\mathrm{Her} 2+$ & 1364 (8.9\%) \\
\hline & Unknown & 817 (5.3\%) & & ER-Her2+ & 1198 (7.8\%) \\
\hline \multirow[t]{4}{*}{ Her2 } & Positive & $2589(16.8 \%)$ & & Triple negative & 1596 (10.4\%) \\
\hline & Negative & $11329(73.7 \%)$ & & Unknown & 2106 (13.7\%) \\
\hline & Equivocal & $533(3.5 \%)$ & & & \\
\hline & Unknown & $931(6.1 \%)$ & Total & & 15382 \\
\hline
\end{tabular}

ER: estrogen receptor, PR: progesterone receptor, Her2: Her2Neu receptor.

Table 6.2 Local recurrence as a first event overall and in subtypes and hazard ratio compared to $\mathrm{ER}+\mathrm{PR}+\mathrm{Her}$ - on univariable Cox regression

\begin{tabular}{lrccc}
\hline & $\mathrm{N}=$ & 5-year LR as a first event & $\mathrm{HR}(95 \% \mathrm{Cl})$ & $\mathrm{p}$-value \\
\hline Total/overall & 15382 & $3.8 \%$ & Ref & \\
ER+PR+Her2- & 7296 & $2.8 \%$ & $1.155(0.839-1.589)$ & 0.377 \\
ER+PR-Her2- & 1822 & $3.1 \%$ & $1.096(0.766-1.569)$ & 0.616 \\
ER+Her2+ & 1364 & $3.0 \%$ & $1.863(1.357-2.558)$ & $<0.001$ \\
ER-Her2+ & 1198 & $4.7 \%$ & $3.871(3.073-4.876)$ & $<0.001$ \\
Triple negative & 1596 & $9.5 \%$ & & \\
\hline
\end{tabular}

Overall comparison Log Rank (Mantel Cox): Chi-square (4)=166.039, p<0.001 


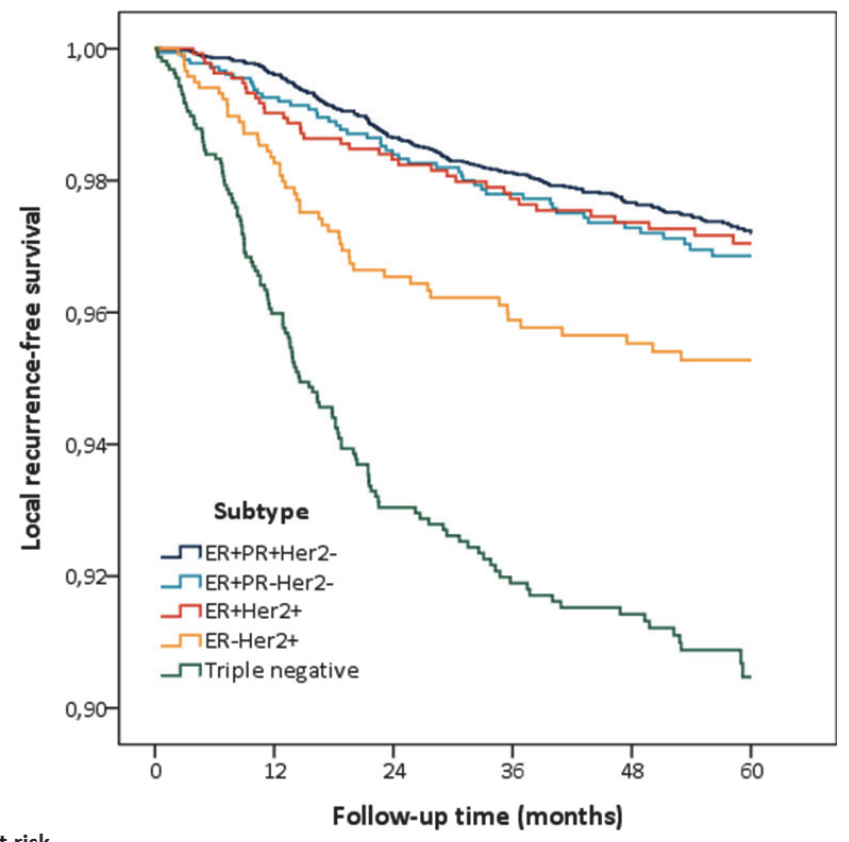

$\mathrm{N}$ at risk

$\begin{array}{lcccccc}\text { ER+PR+Her2- } & 7296 & 6953 & 6522 & 6054 & 5477 & 2600 \\ \text { ER+PR-Her2- } & 1822 & 1668 & 1537 & 1398 & 1251 & 575 \\ \text { ER+Her2+ } & 1364 & 1294 & 1204 & 1114 & 1037 & 678 \\ \text { ER-Her2+ } & 1198 & 1078 & 935 & 840 & 778 & 502 \\ \text { Triple negative } & 1596 & 1318 & 1119 & 996 & 904 & 377\end{array}$

Figure 6.1 Kaplan-Meier plot of local recurrence as a first event within 5 years after diagnosis, in different subtypes of breast cancer

\section{Incidence of LR after mastectomy in Her2+ tumors with and without}

\section{trastuzumab}

Of 1364 ER+Her2+ tumors and 1198 ER-Her2+ tumors, 853 (62.5\%) and 857 (71.5\%) were treated with chemotherapy, and 751 (55\%) and 745 (62.2\%) with trastuzumab. If chemotherapy was administered (suggesting that trastuzumab was also indicated), $86.9 \%$ of ER+Her2+ and $86.6 \%$ of ER-Her2+ tumors also received trastuzumab. LR as a first event occurred in $2.5 \%$ of ER+Her2+ and $4.7 \%$ of ER-Her2+ tumors (Table 6.3). The incidence of LR was significantly lower in patients treated with both chemotherapy and trastuzumab than treatment with chemotherapy alone $(2.0 \%$ vs. $6.0 \%$ in ER+Her2+, $p=0.020$; and $3.5 \%$ vs $6.9 \%$ in ER-Her2+, $p=0.047$ ). The group treated without chemotherapy and trastuzumab is heterogeneous (either no indication or contraindication for systemic therapy) and the group treated with trastuzumab alone consisted of only 13 patients. 
Table 6.3 Local recurrence as a first event in ER+Her2+ and ER-Her2+ patients with and without trastuzumab

\begin{tabular}{|c|c|c|c|c|}
\hline & $\mathrm{ER}+\mathrm{Her} 2+$ & 5-year LR as $1^{\text {st }}$ event & ER-Her2+ & 5-year LR as $1^{\text {st }}$ event \\
\hline Chemotherapy + trastuzumab & $741(54.3 \%)$ & $2.0 \% *$ & 742 (61.9\%) & $3.5 \%^{\#}$ \\
\hline Chemotherapy only & $112(8.2 \%)$ & $6.0 \% *$ & $115(9.6 \%)$ & $6.9 \%$ \\
\hline Trastuzumab only & $10(0.7 \%)$ & $14.3 \%(n=1)$ & $3(0.3 \%)$ & $0 \%$ \\
\hline No chemotherapy/trastuzumab & $501(36.7 \%)$ & $3.6 \%$ & $338(28.2 \%)$ & $6.9 \%$ \\
\hline Total & 1364 & $2.5 \%$ & 1198 & $4.7 \%$ \\
\hline
\end{tabular}

* Chemotherapy\&trastuzumab vs. chemotherapy only: Log Rank (Mantel-Cox) 5.411, p=0.020. \# Chemotherapy\&trastuzumab vs chemotherapy only: Log Rank (Mantel-Cox) 3.928, p=0.047

\section{Predictors for LR after mastectomy as a first event in the overall population}

To assess predictors for LR after mastectomy, several factors were analyzed using univariable (Supplement Table S6.2) and multivariable Cox regression (Table 6.4). The proportional hazards assumption was met for all but two variables included in the multivariable model, namely endocrine therapy and grade.

In the overall population, most factors were significantly associated with LR. The strongest independent predictors were endocrine therapy (protective, $\mathrm{HR} 0.29[95 \% \mathrm{Cl}$ 0.23-0.36]), >3 positive nodes (higher risk compared to 1-3, HR 2.29[1.63-3.21]) and T4 tumor (higher risk compared to T0-1, HR 5.50[3.05-8.38]).

The effect of age was not consistent; only patients aged 40-49 had slightly more LRs than patients $\geq 60$ (HR 1.55[1.17-2.07]).

\section{Multivariable analysis: predictors for LR after mastectomy as a first event per subtype}

The strongest independent predictors for $L R$ on multivariable analysis varied between subtypes (Table 6.4). For three subtypes (ER+PR-Her2-, ER+Her2+, and ER-Her2+), the absolute number of LR was low, leading to wide confidence intervals.

For ER+PR+Her2- breast cancer, the strongest factors were radiation therapy (protective, HR 0.28[0.14-0.54]), endocrine therapy (protective, HR 0.36[0.23-0.56]), and $>3$ positive nodes (higher risk vs. 1-3 nodes, HR 2.74[1.40-5.33]).

For ER+PR-Her2- tumors, the strongest predictors were radiation therapy (protective, HR 0.28 [0.09-0.91]), endocrine therapy (protective, HR 0.34 [0.16-0.75]), and no positive nodes (protective vs. 1-3 nodes, HR 0.31[0.14-0.67]).

In ER+Her2+ tumors, significant factors were trastuzumab (protective, HR 0.26[0.08$0.83]$ ) and endocrine therapy (protective, HR 0.33[0.13-0.87]).

In ER-Her2+ tumors, the only significant predictor was age $40-49$ versus $\geq 60$ (higher risk, HR 2.69 [1.03-7.04]). 


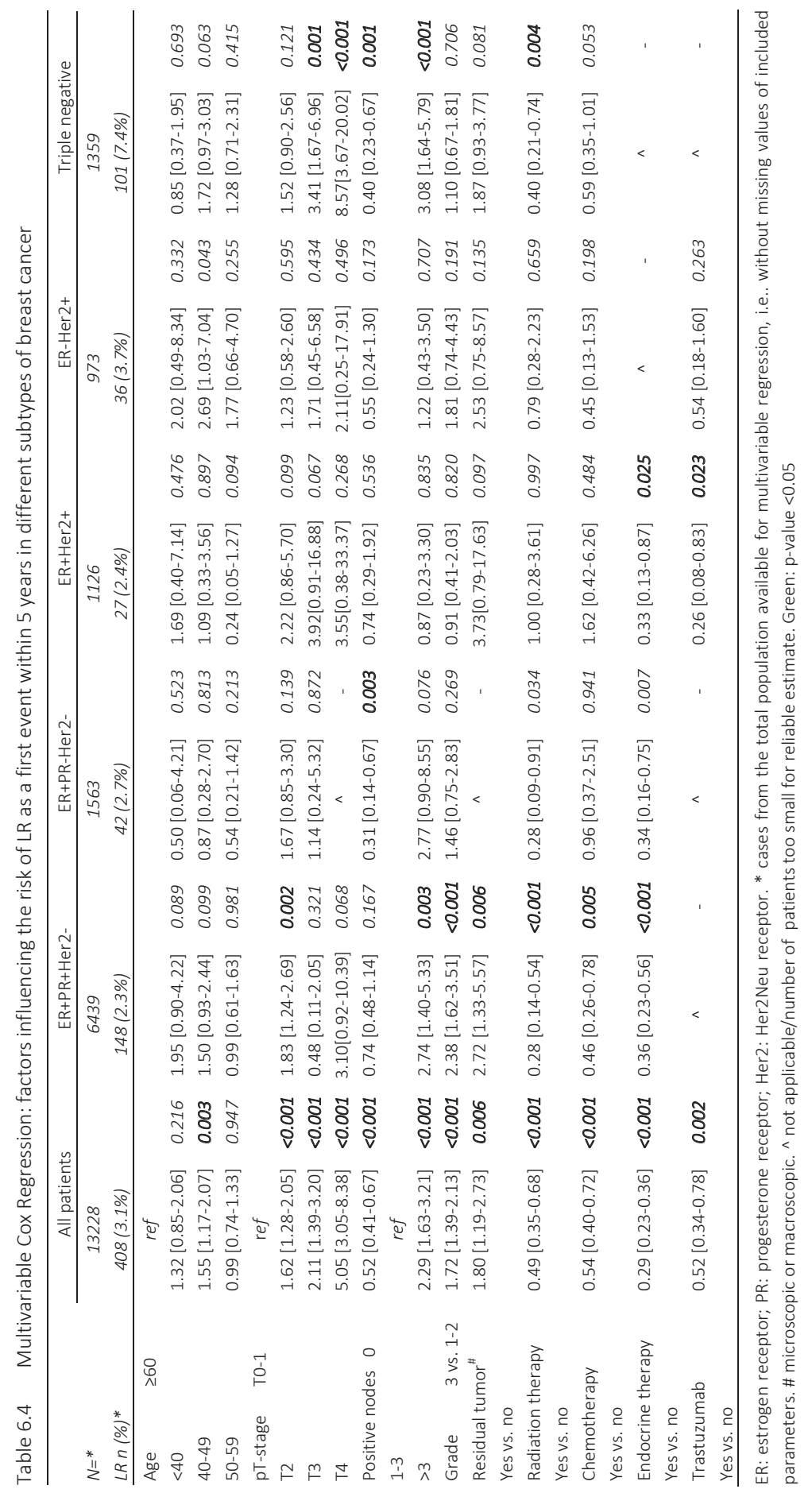


In triple negative tumors, the strongest significant factors were T-stage, both T3 tumor compared to T0-1 (higher risk, HR 3.41[1.67-6.96]) and T4 tumor compared to T0-1 (higher risk, HR 8.57[3.67-20.02]); and the amount of affected lymph nodes: 0 versus 1-3 nodes was protective (HR 0.40[0.23-0.67]) and >3 vs. 1-3 positive nodes increased the risk (HR 3.08[1.64-5.79]).

\section{Multivariable analysis: predictors with different effects in different subtypes}

Some predictors had different effects in different subtypes. PMRT was significantly protective in all subtypes, except for Her2+ subtypes, which showed HRs around 1 and broad confidence intervals. This reflects little or no effect on LR and/or lack of precision of the model as a result of a low number of events. A similar pattern was seen for chemotherapy in ER+PR-Her2- and ER+Her2+ tumors.

A significantly increased risk of LR with higher T-stage was seen overall, but the higher risk for T2 and T3 tumors compared to T0-1 tumors was not significant in most subtypes. In the largest group, ER+PR+Her2- tumors, T3 tumors even showed a non-significant risk reduction compared to T0-1 tumors. In contrast, in triple negative tumors, T3 or T4 stage was a strong significant risk factor for $L R$.

More affected nodes were associated with significantly more LR in the overall population. However, ER+PR+Her2- tumors with 1-3 positive nodes did not have more LRs than patients without positive nodes. In contrast, in triple negative breast cancers, the number of affected nodes was a strong predictor for LR on this multivariable analysis. Grade 3 was associated with an increased risk of LR in the overall population and ER+PR+Her2-tumors, but was not significant in the other subtypes.

\section{Discussion}

This study of 15382 breast cancers treated with mastectomy in The Netherlands in 2005-2008, showed that LR as a first event within 5 years after mastectomy occurred in $3.8 \%$. The incidence varied between subtypes; fewest LR occurred in ER+PR+Her2(2.8\%) and most in triple negative tumors (9.5\%). Significantly fewer LRs occurred in patients with Her2+ tumors treated with both chemotherapy and trastuzumab than patients treated with chemotherapy alone (2.0\% vs. $6.0 \%$ in ER+Her $2+; 3.5 \%$ vs. $6.9 \%$ in ER-Her2+). The strongest independent predictors for LR in the overall population were endocrine therapy (protective), >3 positive lymph nodes, and T4-stage. The importance of risk factors varied between subtypes, most notably the number of positive nodes, radiation therapy, and $\mathrm{T}$-stage. 
Some of the findings suggest that current guidelines regarding more aggressive local therapy, based on traditional characteristics without considering subtype, may be inappropriate. Her2+ tumors had fewer LRs than described in earlier publications ${ }^{13}$ and ER+Her2+ tumors showed no more LRs than ER+Her2- tumors. This illustrates the protective effect of trastuzumab on local endpoints ${ }^{14-16}$ and the difference in biology of ER+Her2+ and ER-Her2+ tumors. The protective effect of PMRT on LR was not seen in Her2+ tumors in this study, resulting from either few events (low precision of the model) and/or little or no effect of PMRT on LR.

The exact relation between Her2+, trastuzumab, and sensitivity to PMRT has not been elucidated. In this study, the protective effect of PMRT was significant in all subtypes except Her2+ tumors, after correction for trastuzumab. Resistance to radiotherapy in Her2+ tumors has been described, e.g. in a post-hoc review of the Danish trials ${ }^{13}$, conducted before introduction of trastuzumab. This showed that generally Her2+ tumors did not have fewer LRR after PMRT, although ER-Her2+ tumors did. Further, a recent study found a non-significant reduction in LRR after PMRT for Her2+ tumors treated without trastuzumab, no LRRs were seen when both trastuzumab and PMRT were used. ${ }^{17}$ Another retrospective analysis of two cohorts treated with and without trastuzumab ${ }^{14}$ showed that trastuzumab reduced LRR in women receiving PMRT but not in women not receiving PMRT. These studies were limited by few events and small patient numbers receiving each combination of treatments (PMRT+trastuzumab, trastuzumab only, PMRT only). This limits valid estimation of any interaction between Her2, trastuzumab, and radiosensitivity. A preclinical study ${ }^{18}$ showed DNA repair in Her2+ tumors after radiation, but addition of a Her2 antibody diminished DNA repair, thus potentially increasing the effect of radiation. The precise interaction between Her2 overexpression, trastuzumab, and radiation is unclear, although earlier publications suggest radioresistance of Her2+ tumors (without trastuzumab) and potentially increased radiosensitivity by trastuzumab.

Furthermore, this study suggests that 1-3 affected nodes is not a risk factor for LR in $\mathrm{ER}+\mathrm{PR}+\mathrm{Her} 2$ - tumors, but it is in triple negative tumors. Having 1-3 positive nodes is often considered a sign of intermediate LR risk. These results show that "intermediate risk" may not be the same for all patients, which is important when identifying risk groups. Additionally, younger age was not consistently associated with more LRs. Younger age is commonly considered an indication for radiation therapy in guidelines, although the evidence regarding higher risk of LR or more benefit of PRMT is scarce. ${ }^{1-3}$ 
A strength of this study is the large patient number in this comprehensive, nationwide database. Many databases are too small to perform multivariable analysis, particularly within subtypes of breast cancer.

A weakness is potential indication bias. For example, more positive nodes might be associated with more LR, but is also an indication for PMRT. This makes etiologic interpretation of HRs difficult. Indication bias is partly overcome by including most indications for therapy in the multivariable model. Secondly, the proportional hazards assumption held for all variables except endocrine therapy and grade, in multivariable analysis of the overall group. As a result, the HR estimates may be somewhat conservative. ${ }^{19}$ As estimating time-dependency of risk factors was not a goal of this study, we did not replicate the modeling including time dependent covariates. Additionally, information on LVI was not available, nor was Ki67. ${ }^{7,8}$ Further, more than five years follow-up might be necessary for ER+tumors, as these are associated with late recurrences, 8 although the effect of Her2 status, targeted treatment, modern chemotherapy, and endocrine treatment on late recurrences is unknown. In this study, the Kaplan-Meier curve showed a constant rate of LR until 5 years for ER+ tumors. Finally, even in this large database, the number of events was small in some subtypes, limiting the precision of the model in ER+PR-Her2-, ER+Her2+, and ER-HER2+ tumors. This illustrates that current LR rates are low, and that assessing risk factors and treatment benefit in less common subtypes is difficult, as even in very large cohorts, few events occur. Finally, it illustrates how natural overrepresentation of ER+PR+Her2tumors in trials may obscure different benefits of treatment in less common subtypes.

The results have consequences for LR risk assessment. First, the absolute risk of LR was lower than in the older studies included in the EBCTCG meta-analysis, even in high-risk subtypes. ${ }^{5}$ A lower absolute risk with the same reduction implies lower absolute benefit. This means that the absolute benefit of PMRT might be small, especially in lower-risk subtypes. Secondly, although a high risk in a retrospective analysis does not prove that a subtype would benefit from more aggressive treatment, the differences between subtypes should be considered in randomised studies investigating local treatment, such the SUPREMO-trial ${ }^{20}$, investigating PMRT in intermediate risk patients. Based on the current study, it is likely that the definition of intermediate risk differs per subtype, in addition to varying radiosensitivity and benefit from systemic treatment. Trials investigating local treatment should report results separately for subtypes. Because the low number of events in less common subtypes may limit statistical power, pooling of different trials is essential. 
In conclusion, the overall risk of LR as a first event after mastectomy was $3.2 \%$ and significantly differed between subtypes of breast cancer. Triple negative tumors were at highest risk and ER+PR+Her2- at the lowest. Commonly known risk factors were confirmed, but their importance varied between subtypes. Based on varying absolute risk, risk factors, and potentially different treatment sensitivity, local treatment should be tailored to subtype and trials investigating local treatment should report on potentially different risk profiles and benefit of treatment in breast cancer subtypes. 


\section{References}

1. NABON, Vereniging van Integrale Kankercentra. Breast Cancer: Nation-wide guideline (2.0). November 2012:1-297.

2. Recht A, Edge SB, Solin LJ, et al. Postmastectomy radiotherapy: clinical practice guidelines of the American Society of Clinical Oncology. J Clin Oncol. 2001;19(5):1539-1569.

3. NICE. Early and Locally Advanced Breast Cancer: Diagnosis and Treatment. Full Guideline. (National Collaborating Centre for Cancer, ed.). Velindre NHS Trust, Cardiff, Wales; 2009.

4. Clarke M, Collins R, Darby S, et al. Effects of radiotherapy and of differences in the extent of surgery for early breast cancer on local recurrence and 15-year survival: an overview of the randomised trials. Lancet. 2005;366(9503):2087-2106.

5. EBCTCG, McGale P, Taylor C, et al. Effect of radiotherapy after mastectomy and axillary surgery on 10year recurrence and 20-year breast cancer mortality: meta-analysis of individual patient data for 8135 women in 22 randomised trials. Lancet. 2014;383(9935):2127-2135.

6. van der Heiden-van der Loo M, Ho VKY, Damhuis RAM, et al. Weinig lokaal recidieven na mammachirurgie: goedekwaliteit van de Nederlandse borstkankerzorg. Nederlands tijdschrift voor geneeskunde. 2010;154(28):A1984.

7. Voduc KD, Cheang MCU, Tyldesley S, Gelmon K, Nielsen TO, Kennecke H. Breast cancer subtypes and the risk of local and regional relapse. J Clin Oncol. 2010;28(10):1684-1691.

8. Colleoni M, Sun Z, Price KN, et al. Annual Hazard Rates of Recurrence for Breast Cancer During 24 Years of Follow-Up: Results From the International Breast Cancer Study Group Trials I to V. J Clin Oncol. 2016; 34(9):927-935.

9. Lowery AJ, Kell MR, Glynn RW, Kerin MJ, Sweeney KJ. Locoregional recurrence after breast cancer surgery: a systematic review by receptor phenotype. Breast Cancer Res Treat. 2012;133(3):831-841.

10. Witteveen A, Vliegen IMH, Sonke GS, Klaase JM, IJzerman MJ, Siesling S. Personalisation of breast cancer follow-up: a time-dependent prognostic nomogram for the estimation of annual risk of locoregional recurrence in early breast cancer patients. Breast Cancer Res Treat. 2015;152(3):627-636.

11. CBO Kwaliteitsinstituut voor de Gezondheidszorg, Vereniging van Integrale Kankercentra. Guideline 'Treatment of breast cancer' (Richtlijn 'Behandeling van het Mammacarcinoom'). 2005. http://www.oncoline.nl/uploaded/FILES/mammacarcinoom/Richtlijn\%20Behandeling\%20van\%20het\%20 Mammacarcinoom\%20oktober\%202005.pdf.

12. Moossdorff M, van Roozendaal LM, Strobbe LJ, et al. Maastricht Delphi Consensus on Event Definitions for Classification of Recurrence in Breast Cancer Research. Journal of the National Cancer Institute. 2014;106(12):dju288-dju288.

13. Kyndi M, Sørensen FB, Knudsen H, et al. Estrogen receptor, progesterone receptor, HER-2, and response to postmastectomy radiotherapy in high-risk breast cancer: the Danish Breast Cancer Cooperative Group. J Clin Oncol. 2008;26(9):1419-1426.

14. Lanning RM, Morrow M, Riaz N, et al. The Effect of Adjuvant Trastuzumab on Locoregional Recurrence of Human Epidermal Growth Factor Receptor 2-Positive Breast Cancer Treated with Mastectomy. Ann Surg Oncol. 2015;22(8):2517-2525.

15. Dahabreh IJ, Linardou H, Siannis F, Fountzilas G, Murray S. Trastuzumab in the adjuvant treatment of early-stage breast cancer: a systematic review and meta-analysis of randomized controlled trials. Oncologist. 2008;13(6):620-630.

16. Romond EH, Perez EA, Bryant J, et al. Trastuzumab plus adjuvant chemotherapy for operable HER2positive breast cancer. N Engl J Med. 2005;353(16):1673-1684.

17. Tseng YD, Uno H, Hughes ME, et al. Biological Subtype Predicts Risk of Locoregional Recurrence After Mastectomy and Impact of Postmastectomy Radiation in a Large National Database. Int J Radiat Oncol Biol Phys. 2015;93(3):622-630.

18. Pietras RJ, Poen JC, Gallardo D, Wongvipat PN, Lee HJ, Slamon DJ. Monoclonal antibody to HER2/neureceptor modulates repair of radiation-induced DNA damage and enhances radiosensitivity of human breast cancer cells overexpressing this oncogene. Cancer Res. 1999;59(6):1347-1355.

19. Harrell FE. Regression Modeling Strategies. Springer Science \& Business Media; 2001. 
20. Kunkler IH, Canney P, van Tienhoven G, Russell NS, MRC/EORTC (BIG 2-04) SUPREMO Trial Management Group. Elucidating the role of chest wall irradiation in "intermediate-risk" breast cancer: the MRC/EORTC SUPREMO trial. Clin Oncol (R Coll Radiol). 2008;20(1):31-34. 


\section{Supplement tables}

Table S6.1 Adjuvant treatment in different subtypes

\begin{tabular}{llllll}
\hline & ER+PR+Her2- & ER+PR-Her2- & ER+Her2+ & ER-Her2+ & Triple negative \\
& 7296 & 1822 & 1364 & 1198 & 1596 \\
\hline Radiation therapy & $1983(27.2 \%)$ & $559(30.7 \%)$ & $473(34.7 \%)$ & $500(41.7 \%)$ & $566(35.5 \%)$ \\
Chemotherapy & $2952(40.5 \%)$ & $694(38.1 \%)$ & $853(62.5 \%)$ & $857(71.5 \%)$ & $1026(64.3 \%)$ \\
Endocrine therapy & $5042(69.1 \%)$ & $1272(69.8 \%)$ & $1083(79.4 \%)$ & $87(7.3 \%)$ & $59(3.7 \%)$ \\
Trastuzumab & $22(0.3 \%)$ & $6(0.3 \%)$ & $751(55.1 \%)$ & $745(62.2 \%)$ & $11(0.7 \%)$ \\
\hline
\end{tabular}




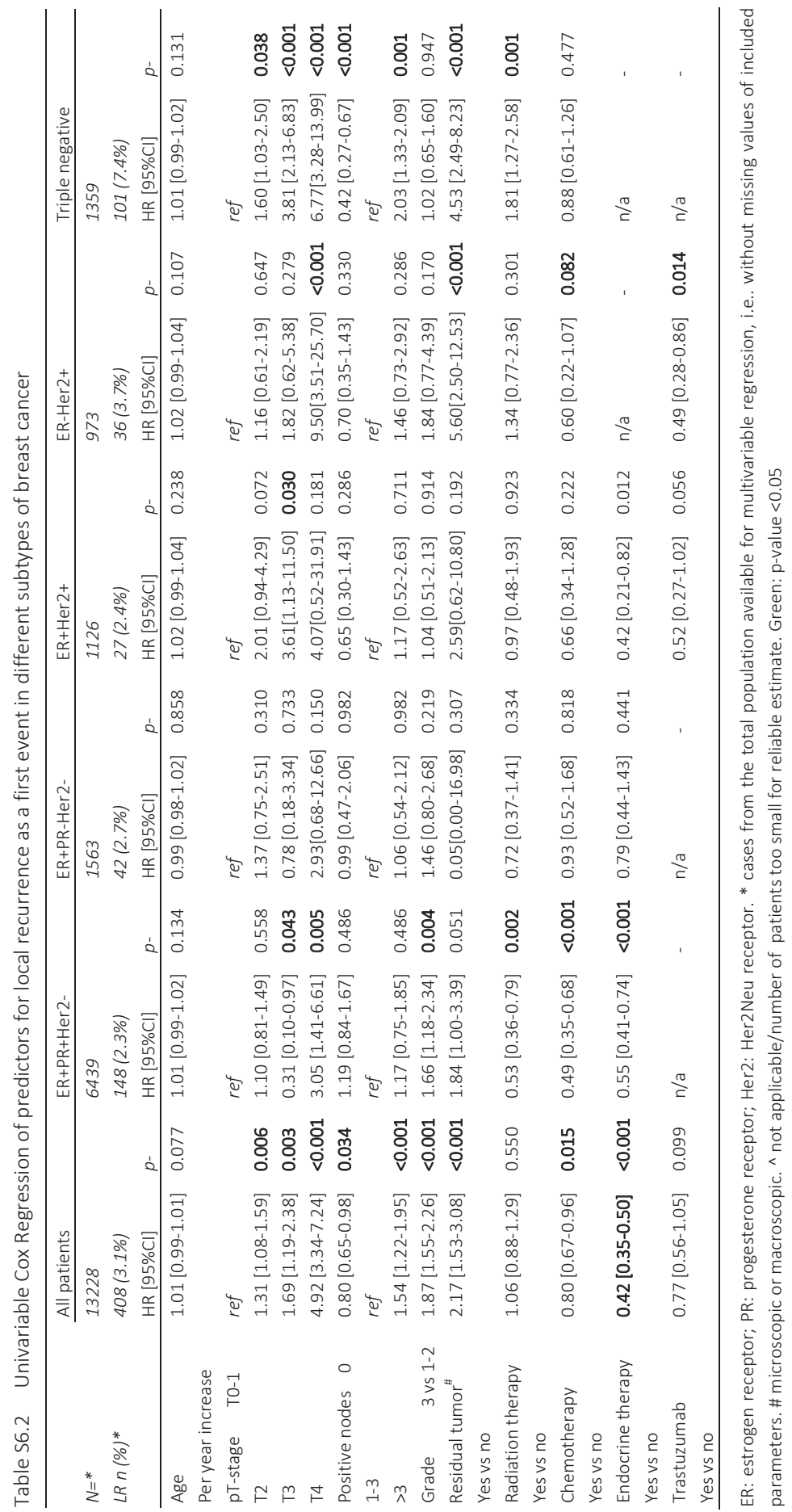




\section{Chapter 7}

Conditional local recurrence: The effect of event-free years on the risk of 5-year local recurrence in different subtypes of breast cancer

Moossdorff M, van Nijnatten T, Vane M, van Maaren MC, Goorts B, Heuts EM, Strobbe LJA, Smidt ML

Submitted 


\section{Abstract}

\section{Introduction}

After treatment for breast cancer, follow-up consists of physical examination and mammography for at least five years, to detect local and regional recurrence. The chance of getting such a recurrence may decrease after event-free time, perhaps even to the point that follow-up is no longer useful. The aim of this study is to determine the risk of local recurrence (LR) as a first event until 5 years after diagnosis, conditional on being event-free for 1, 2, 3, and 4 years.

\section{Methods}

The Netherlands Cancer Registry contains data of all newly diagnosed cancers in the Netherlands. All new epithelial breast cancers without distant metastasis, diagnosed between 2005 and 2008 were included. LR risk was calculated with Kaplan-Meier analysis, overall and for different breast cancer subtypes. Conditional LR (assuming $x$ event-free years) was determined by selecting patients without an event at $x$ years, and calculating the risk of LR within 5 years after diagnosis.

\section{Results}

Five-year follow-up was available for 34,453 patients. Overall, 5-year LR as a first event occurred in $3.0 \%$. This risk varied for different subtypes and was highest for triple negative (6.8\%) and lowest for ER+PR+Her2- (2.2\%) tumors. After 1, 2, 3, and 4 eventfree years, the average risk of LR before the end of regular follow-up (5 years after diagnosis) decreased from $3.0 \%$ to $2.4 \%, 1.6 \%, 1.0 \%$, and $0.6 \%$. The risk decreased in all subtypes and the effect was most pronounced in subtypes with the highest baseline risk (ER-Her2+ and triple negative breast cancer). After 3 event-free years, the risk of LR in the next two years (i.e. before 5 years after diagnosis/end of regular follow-up) was $1 \%$ or less in all subtypes except triple negative tumors (1.6\%).

\section{Conclusion}

The risk of 5-year LR as a first event was low overall (3.0\%). This risk decreased even further with the number of event-free years. After 3 event-free years, the overall risk was $1 \%$. This improvement in prognosis is reassuring to patients during follow-up. It also suggests that follow-up beyond 3 years may have a low yield of $L R$, both for individual patients and clinical studies using LR as the primary outcome. This can be used as a starting point to tailor follow-up to individual needs. 


\section{Introduction}

Outcomes such as local recurrence (LR) are usually expressed as 5 or 10-year probability from the time of breast cancer diagnosis. However, as time progresses and a patient remains event-free, this initial estimate of local recurrence (or other outcomes) may have improved. Event-free time is usually not considered as a prognostic factor. An estimate of prognosis that takes the recurrence-free interval into account is called conditional survival or recurrence.

Earlier publications have addressed conditional overall and disease-free survival in breast cancer patients, ${ }^{1-3}$ however without focus on local recurrence. Further, these studies were based on older cohorts that differed from current breast cancer patients in several ways: worse baseline prognosis, diagnosis in a time period when breast cancer screening was unavailable, incomplete information on intrinsic subtypes including Her2 status, incomplete use of modern (taxane-based) chemotherapy regimens, and incomplete use of trastuzumab for Her2 overexpressing tumors.

The advantage of calculating conditional local recurrence risks is that individual patients can receive more tailored information about their prognosis, which could be reassuring. Furthermore, this information can also help to determine the optimal follow-up time, both for everyday practice and clinical research. After treatment for breast cancer, follow-up consists of physical examination and mammography for at least five years. Thereafter, recommendations vary with regard to frequency, duration, and required investigations. One of the goals of follow-up is to detect possible local and regional recurrences. ${ }^{4-7}$ Information on conditional local recurrence risk may be used to tailor follow-up to individual needs. Although extended follow-up may be desirable for other goals such as monitoring endocrine therapy and reassurance, a low chance of events may be a reason to shorten follow-up in specific cases. Safely tailoring follow-up to individual patients could improve quality of care by reducing the number of hospital visits and stress. It can also save health care costs, and may also decrease the required time and financial resources for clinical trials if follow-up can be shortened. In order to preserve quality of care, we need to explore which patients may be eligible for this approach.

Earlier studies on conditional overall and disease-free survival demonstrated the greatest improvement of prognosis (in other words: greatest reduction of the chance of recurrence and death) for patients with the worst prognosis at baseline. This is in line with conditional survival studies for other types of cancer. ${ }^{8-11}$ As we hypothesize this may also be the case for LR risk in breast cancer, the role of biologic subtype as prognostic factor may be of interest, in addition to traditional prognostic factors such as tumor size and nodal status. Different subtypes show different patterns of recurrence. ${ }^{12}$ It is 
plausible that the prognostic differences between subtypes depend, among others, on contemporary chemotherapy and trastuzumab. Knowing the effect of event-free years on LR risk in different subtypes could allow tailoring of follow-up, both for clinical practice and trials using LR as an endpoint.

This study aims to determine the risk of LR as a first event within 5 years after diagnosis, conditional on having no breast cancer event for $1,2,3$, and 4 years. The results will be presented separately for ER+PR+Her2-, ER+PR-Her2-, ER+Her2+, ER-Her2+, and triple negative tumors.

\section{Methods}

\section{Data collection}

The Netherlands Cancer Registry (NCR) collects data on all newly diagnosed cancer patients in all hospitals in the Netherlands from 1989 onward. For the years 2005-2008, both five-year follow-up on recurrences and information on Her2 status and treatment with trastuzumab are available. Trained data registrars of the Netherlands Comprehensive Cancer Organisation (IKNL) obtain data on tumor characteristics, as well as surgical, radiation, and systemic treatment from patients' records. Tumor topography and morphology were coded according to the International Classification of Diseases for Oncology (ICD-O, $3^{\text {rd }}$ edition ${ }^{13}$ ), and staging was coded according to the tumor, node and metastasis (TNM) classification system (AJCC/UICC, $6^{\text {th }}$ edition ${ }^{14}$ ). For a period of 5 years after diagnosis, the first breast cancer event was registered (LR, new primary ipsilateral breast cancer, contralateral breast cancer, regional recurrence, or distant recurrence).

\section{Included patients}

From the NCR database, all new invasive epithelial breast cancers diagnosed between 2005 and 2008, of which 5-year follow-up was complete, were included. Patients with distant metastasis at (or within 91 days of) diagnosis were excluded.

\section{Treatment according to guideline}

Patients were treated according to the Dutch national breast cancer guideline of $2005 .{ }^{15}$ Local treatment consisted of breast conserving therapy (lumpectomy and whole breast irradiation) or mastectomy. Post-mastectomy chest wall irradiation was recommended for positive margins, involvement of the pectoralis muscle or skin (T4 tumors), and was considered individually for $\mathrm{pT} 3$ tumors. Locoregional radiation was performed for $\geq \mathrm{pN} 2$ or involvement of upper medial axillary nodes. Recommended dose was 45-50 Gy in 5 
weeks, or 60-70 Gy in 6 or 7 weeks in case of residual tumor. Lymph node involvement was assessed with sentinel lymph node biopsy (SLNB) for clinically node negative patients according to physical examination and biopsy/fine needle aspiration. Axillary ultrasound was common but not mandatory. Contraindications for SLNB at that time were multiple tumors, >T2, and previous axillary surgery. If SLNB was contraindicated, or if positive lymph nodes were identified either preoperatively or by SLNB, an axillary lymph node dissection (ALND) was performed.

The indication for systemic treatment depended on nodal involvement, tumor size, grade, receptor status, and age. In $\mathrm{N}+$ breast cancer, endocrine therapy was recommended for all patients with ER+ and/or PR+ tumors. Chemotherapy was advised for $\mathrm{N}+$ breast cancer in all premenopausal women and in women $<70$ years old with ERand PR-tumors. In postmenopausal women aged 50-59 with ER+PR+ and N+tumors, chemotherapy was considered if patients were in good physical condition, and in women aged 60-69 only if 4 or more of nodes were involved.

For NO breast cancer, systemic therapy (both chemotherapy and endocrine therapy for ER+ or PR+ tumors and chemotherapy for ER-PR-tumors) was considered for patients $\leq 35$ years (except grade I tumors $\leq 1 \mathrm{~cm}$ ), and for patients $>35$ years with tumors $\geq 3 \mathrm{~cm}$, or $\geq 1 \mathrm{~cm}$ and grade $\mathrm{II}$, or $\geq 2 \mathrm{~cm}$ and grade II. Standard chemotherapy consisted of 5 courses of FEC (fluorouracil/epirubicin/cyclophosphamide) or 6 courses of TAC (docetaxel/doxorubicin/cyclophosphamide). If chemotherapy was indicated for a Her2 overexpressing tumor, patients were treated with trastuzumab for one year after chemotherapy.

Endocrine therapy consisted of tamoxifen for 5 years for premenopausal women, optionally including LHRH agonist if not postmenopausal after chemotherapy. For postmenopausal women, either an aromatase inhibitor was given for 5 years, or tamoxifen for 2 years, followed by an aromatase inhibitor.

\section{Pathology and approximate subtypes}

Five subtypes of breast cancer were distinguished, namely ER+PR+Her2-, ER+PR-Her2-, ER+Her2+, ER-Her2+, and triple negative tumours. Tumours were considered ER+ and $\mathrm{PR}+$ if more than $10 \%$ of tumour cells showed nuclear staining on immunohistochemistry (IHC). Her2 status was evaluated with at least IHC, in which 3+ was considered positive ( $>10 \%$ of cells with strong intensity circumferential membrane staining) and 0 and $1+$ were considered negative ( $<10 \%$ circumferential membrane staining, or $>10 \%$ with weak intensity membrane staining). In case of a $2+\mathrm{IHC}$ score ( $>10 \%$ circumferential membrane staining with moderate intensity), fluorescence in situ hybridization (FISH) 
was mandatory in addition to IHC. If FISH was used, the result of FISH overruled the result of IHC.

\section{Endpoints}

The primary endpoint was (conditional) LR as a first event within 5 years after diagnosis. LR was defined as any invasive breast cancer in the ipsilateral breast (including skin, biopsy tract and surgical scar) or on the ipsilateral thoracic wall including the mastectomy scar, i.e. both LR and new primary ipsilateral breast cancer were counted as LR. ${ }^{16}$ Events between 0 and 91 days after diagnosis were regarded as synchronous with the original tumour. Patients were censored at the date of their first event (see data collection above), at the last date of follow-up, or at the date of death. If another event occurred within 91 days of the first recurrence, this was considered synchronous with the first event, and also counted as a first recurrence.

Statistical analyses were performed using SPSS [IBM Corporation, version 23.0.0.0]. Kaplan-Meier analysis was used to determine 5-year LR as a first event, for the overall population and separately for five approximate subtypes of breast cancer. To check whether there was an effect of subtype independent of tumor and treatment characteristics, multivariable Cox regression was performed. Variables that were significantly associated with LR on univariable analysis, as well as those known to influence the risk of LR were included in the multivariable analysis. Missing values were disregarded, not imputed. Conditional LR (assuming $x$ event-free years) was determined by selecting patients without an event at $x$ years, and calculating the risk of LR within 5 years after diagnosis for this selection.

\section{Results}

\section{Baseline characteristics}

In total, the database contained 34.453 new breast cancers diagnosed between 2005 and 2008, of which 5-year follow-up was available. Median age was 59.0 years [range: 20-100]. Of these patients, 15.382 (44.6\%) were treated with mastectomy, 19.071 $(55.4 \%)$ with breast conserving therapy. The majority of tumors were ER+PR+Her2(51.6\%), 11.4\% were ER+PR-Her2-, 7.8\% were ER+Her2+, 5.5\% ER-Her2+, and 10.5\% triple negative. Of 4548 (13.2\%) tumors, subtype was unknown (Table 7.1). 
Table 7.1 Baseline characteristics

\begin{tabular}{|c|c|c|c|c|c|}
\hline Median age (range) & & $59.0[20-100]$ & Morphology & Ductal & $25833(75.0 \%)$ \\
\hline \multirow[t]{6}{*}{ pT-stage } & TO & $240(0.7 \%)$ & & Lobular & 3753 (10.9\%) \\
\hline & $\mathrm{T} 1$ & $20759(60.3 \%)$ & & $\begin{array}{l}\text { Mixed } \\
\text { ductal/lobular }\end{array}$ & $2122(6.1 \%)$ \\
\hline & $\mathrm{T} 2$ & 11547 (33.5\%) & & Other & $2745(8.0 \%)$ \\
\hline & T3 & $1036(3.0 \%)$ & Positive margins & No & 32504 (94.3\%) \\
\hline & T4 & $343(1.0 \%)$ & & Microscopic & $1398(4.1 \%)$ \\
\hline & $T x$ & $528(1.5 \%)$ & & Macroscopic & $49(0.1 \%)$ \\
\hline \multirow[t]{5}{*}{ pN-stage } & NO & $20884(60.6 \%)$ & & Unknown & $502(1.5 \%)$ \\
\hline & N1 & 9157 (26.6\%) & Breast surgery & Mastectomy & $15382(44.6 \%)$ \\
\hline & N2 & 2533 (7.3\%) & & $\mathrm{BCT}$ & 19071 (55.4\%) \\
\hline & N3 & $1403(4.1 \%)$ & Radiation therapy & Yes & $23128(67.1 \%)$ \\
\hline & $\mathrm{Nx}$ & $476(1.4 \%)$ & & No & 11325 (32.9\%) \\
\hline \multirow[t]{4}{*}{ Grade } & 1 & 7449 (21.6\%) & Chemotherapy & Yes & 13392 (38.9\%) \\
\hline & 2 & 14275 (41.5\%) & & Neoadjuvant & $1708(5.0 \%)$ \\
\hline & 3 & 10204 (29.6\%) & & No & 21061 (61.1\%) \\
\hline & Unknown & 2525 (7.3\%) & $\begin{array}{l}\text { Endocrine therapy for } Y \\
\text { ER+ tumors }\end{array}$ & Yes & $\begin{array}{l}15281 / 27628 \\
(55.3 \%)\end{array}$ \\
\hline \multirow[t]{3}{*}{$\overline{E R}$} & Positive & $27628(80.2 \%)$ & $\begin{array}{l}\text { Trastuzumab } \\
\text { Her2+ tumors }\end{array}$ & Yes & $\begin{array}{l}2584 / 4638 \\
(55.7 \%)\end{array}$ \\
\hline & Negative & $6314(18.3 \%)$ & $\begin{array}{ll}\text { Trastuzumab } & \text { for Y } \\
\text { Her2+ } & \text { tumors } \\
\text { receiving } & \\
\text { chemotherapy* } & \\
\end{array}$ & Yes & $\begin{array}{l}2560 / 2926 \\
(87.5 \%)\end{array}$ \\
\hline & Unknown & $511(1.5 \%)$ & Subtype & $\mathrm{ER}+\mathrm{PR}+\mathrm{Her} 2-$ & 17770 (51.6\%) \\
\hline \multirow[t]{3}{*}{$P R$} & Positive & 21750 (63.1\%) & & ER+PR-Her2- & 3930 (11.4\%) \\
\hline & Negative & 10960 (31.8\%) & & ER+Her2+ & 2689 (7.8\%) \\
\hline & Unknown & $1743(5.1 \%)$ & & ER-Her2+ & 1897 (5.5\%) \\
\hline \multirow[t]{4}{*}{ Her2 } & Positive & $4638(13.5 \%)$ & & Triple negative & 3619 (10.5\%) \\
\hline & Equivocal & 1092 (3.2\%) & & Unknown & $4548(13.2 \%)$ \\
\hline & Negative & $26693(77.4 \%)$ & & & \\
\hline & Unknown & $2030(5.9 \%)$ & Total & & 34453 \\
\hline
\end{tabular}

ER: estrogen receptor, PR: progesterone receptor, BCT: breast conserving therapy. * If a patient with a Her2+ tumor was eligible for chemotherapy, this patient was also eligible for trastuzumab. \# Included in chemotherapy 'yes', percentage of total

\section{Local recurrence as a first event within 5 years in different subtypes}

The incidence of LR as a first event within 5 years of diagnosis varied between the subtypes of breast cancer (Table 7.2, Figure 7.1). Incidence was highest in triple negative tumors (5.6\%) and lowest in ER+PR+Her2-tumors (1.9\%). The difference between the subtypes was significant, except for the difference between ER+PR+Her2- and ER+PRHer2- (2.2\% vs. 2.4\%, p=0.329); and ER+PR-Her2- and ER+Her2+ (2.4\% vs. 2.8\%, $p=0.342)$. The difference between $E R+P R+H e r 2-(2.2 \%)$ and ER+Her2+ (2.8\%) was significant $(p=0.046)$. 
Table 7.2 Risk of local recurrence as a first event (Kaplan-Meier survival estimates) within 5 years after diagnosis in different subtypes of breast cancer

\begin{tabular}{lccc}
\hline & $N$ & $\begin{array}{c}\text { 5-year risk of LR at diagnosis } \\
\text { All patients }\end{array}$ & $\begin{array}{c}\text { Significance of difference between the } \\
\text { Kaplan-Meier curves }\end{array}$ \\
\hline Approximate subtypes & 34453 & $3.0 \%$ & \\
\hline $\begin{array}{l}\text { ER+PR+Her2- } \\
17770\end{array}$ & $2.2 \%$ & \}$p=0.329, \chi^{2}=0.954$ \\
$\begin{array}{l}\text { ER+PR-Her2- } \\
\text { ER+Her2+ }\end{array}$ & $2.4 \%$ & \}$p=0.342^{*}, \chi^{2}=0.902$ \\
ER-Her2+ & 1897 & $2.8 \%$ & \}$p<0.001, \chi^{2}=12.599$ \\
Triple negative & 3619 & $4.7 \%$ & \}$p=0.006, \chi^{2}=7.535$ \\
\hline
\end{tabular}

ER: estrogen receptor, PR: progesterone receptor, Her2: Her2Neu receptor. Log Rank (Mantel-Cox) was used to compare significance between the Kaplan-Meier curves. * ER+Her2+ $2.8 \%)$ tumors did not have significantly more LR than ER+PR-Her2- (2.4\%), but ER+Her2+ did have significantly more LR than the most favorable subtype $E R+P R+H e r 2-(2.2 \%), p=0.046, \chi^{2}=3.978$

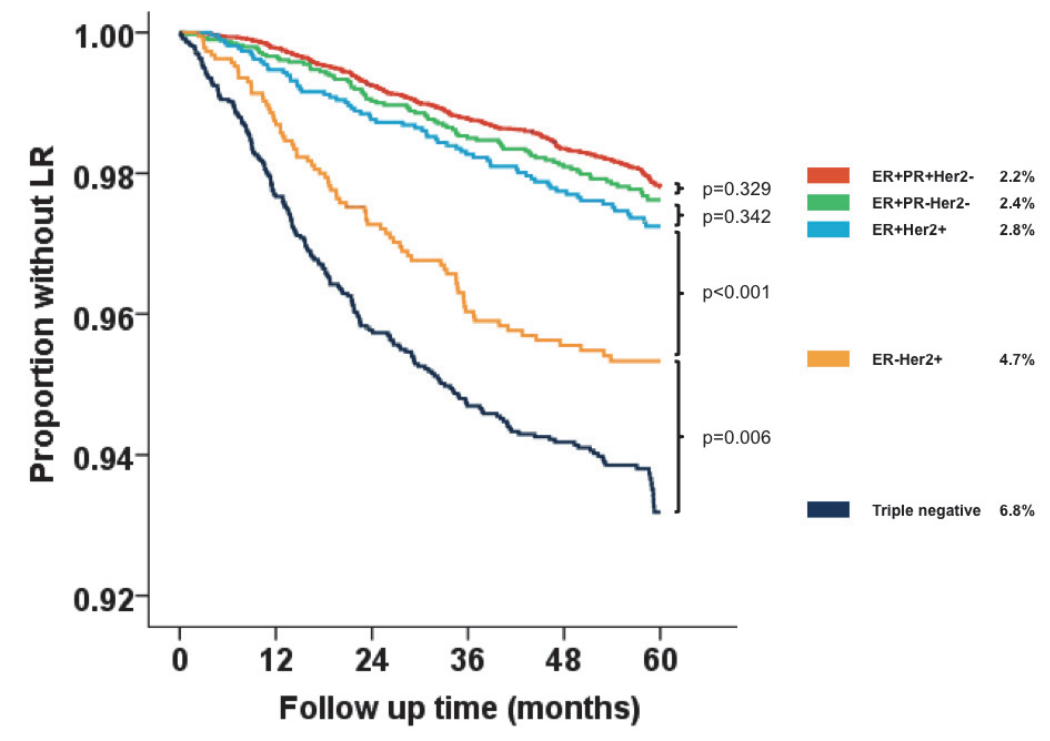

Figure 7.1 Kaplan-Meier estimator plot of risk of local recurrence as a first event within 5 years after diagnosis in different subtypes of breast cancer 


\section{Local recurrence in different subtypes: differences significant on multivariable analysis}

Factors that may influence the risk of LR in different subtypes were selected based on known prognostic significance and/or univariable analysis. When corrected for the selected factors using multivariable Cox regression, the difference in LR between $\mathrm{ER}+\mathrm{PR}+\mathrm{Her} 2$ - tumors and the other subtypes was still significant (p-values $<0.05, \mathrm{HRs}, \mathrm{Cls}$ and $p$-values in Table 7.3), except for the difference between ER+PR+Her2- versus ER+PR-Her2- which has a HR of 0.954 with $p=0.329$. Additionally, after correction for these factors, there was no longer a significant difference in LR between patients treated with mastectomy and breast conserving therapy (HR 1.234, 95\% Cl 0.944-1.614, $\mathrm{p}=0.124)$.

Table 7.3 Multivariable Cox regression to assess the impact of breast cancer subtype on 5-year local recurrence as a first event, corrected for confounding factors

\begin{tabular}{|c|c|c|c|}
\hline & $\mathrm{HR}$ & $95 \% \mathrm{Cl}$ & $p$-value \\
\hline Subtype vs. ER+PR+Her2- & Ref & & \\
\hline ER+PR-Her2- & 1.134 & $0.876-1.467$ & 0.341 \\
\hline ER+Her2+ & 1.535 & $1.120-2.105$ & 0.008 \\
\hline ER-Her2+ & 1.525 & $1.044-2.228$ & 0.029 \\
\hline Triple negative & 2.102 & $1.613-2.740$ & $<0.001$ \\
\hline $\begin{array}{l}\text { Age } \\
\text { Per year increase }\end{array}$ & 0.992 & $0.984-0.999$ & 0.019 \\
\hline $\begin{array}{l}\mathrm{N} \text {-stage } \\
\mathrm{N}+\text { vs. NO }\end{array}$ & 2.152 & $1.785-2.594$ & $<0.001$ \\
\hline $\begin{array}{l}\text { T-stage } \\
\text { T3-4 vs. T1-2 }\end{array}$ & 2.221 & $1.581-3.121$ & $<0.001$ \\
\hline $\begin{array}{l}\text { Grade } \\
3 \text { vs. } 1-2\end{array}$ & 1.530 & $1.254-1.866$ & $<0.001$ \\
\hline $\begin{array}{l}\text { Breast surgery } \\
\text { Mastectomy vs. BCT }\end{array}$ & 1.234 & $0.944-1.614$ & 0.124 \\
\hline $\begin{array}{l}\text { Radiation therapy } \\
\text { No vs. yes }\end{array}$ & 1.575 & $1.216-2.039$ & 0.001 \\
\hline $\begin{array}{l}\text { Chemotherapy } \\
\text { No vs. yes }\end{array}$ & 1.837 & $1.438-2.346$ & $<0.001$ \\
\hline $\begin{array}{l}\text { Endocrine therapy } \\
\text { No vs yes }\end{array}$ & 2.428 & $1.934-3.049$ & $<0.001$ \\
\hline $\begin{array}{l}\text { Trastuzumab } \\
\text { No vs. yes }\end{array}$ & 1.656 & $1.104-2.485$ & 0.015 \\
\hline
\end{tabular}

\section{The effect of event-free years on the risk of local recurrence within} 5 years

For each subtype, the risk of conditional 5-year LR was calculated by selecting patients who were event free (i.e. no local, regional, or distant recurrence, no contralateral breast cancer, and no death) at 12, 24, 36, and 48 months. For each time point and each 
subtype, the risk of LR within 5 years of diagnosis (the end of regular follow-up) was calculated (Table 7.3).

For the overall group, the risk of developing LR before the end of regular follow-up (5 years) was $2.5 \%$. This risk decreased with event-free years, to $2.0 \%, 1.4 \%, 0.9 \%$, and $0.4 \%$ after $1,2,3$, and 4 event-free years (Table 7.4).

This decrease in risk was seen in all subtypes, and was proportionally largest in the subtypes with the highest baseline risk (triple negative and ER-Her2+ tumors). After 3 event-free years, the risk of developing LR before the end of regular follow-up (5 years) was $1 \%$ or less in all subtypes but triple negative tumors (Table 7.4).

Table 7.4 Impact of a number of event-free years on the risk of local recurrence as a first event within 5 years after diagnosis in subtypes of breast cancer

\begin{tabular}{|c|c|c|c|c|c|c|}
\hline & \multirow[b]{2}{*}{$N=$} & \multirow[b]{2}{*}{$\begin{array}{c}\text { Risk of LR at } \\
\text { diagnosis }\end{array}$} & \multicolumn{4}{|c|}{$\begin{array}{c}\text { Risk of LR within } 5 \text { years after diagnosis, assuming x event- } \\
\text { free years - events/persons at risk (\%) }\end{array}$} \\
\hline & & & $\begin{array}{l}\text { After } 1 \text { event- } \\
\text { free year }\end{array}$ & $\begin{array}{l}\text { After } 2 \text { event- } \\
\text { free years }\end{array}$ & $\begin{array}{l}\text { After } 3 \text { event- } \\
\text { free years }\end{array}$ & $\begin{array}{l}\text { After } 4 \text { event- } \\
\text { free years }\end{array}$ \\
\hline All patients & 34453 & $3.0 \%$ & $2.4 \%$ & $1.6 \%$ & $1.0 \%$ & $0.6 \%$ \\
\hline \multicolumn{7}{|c|}{ Approximate subtypes } \\
\hline ER+PR+Her2- & 17770 & $2.2 \%$ & $2.0 \%$ & $1.5 \%$ & $1.0 \%$ & $0.6 \%$ \\
\hline ER+PR-Her2- & 3930 & $2.4 \%$ & $2.0 \%$ & $1.4 \%$ & $0.9 \%$ & $0.5 \%$ \\
\hline $\mathrm{ER}+\mathrm{Her} 2+$ & 2689 & $2.8 \%$ & $2.2 \%$ & $1.5 \%$ & $1.0 \%$ & $0.4 \%$ \\
\hline ER-Her2+ & 1897 & $4.7 \%$ & $3.4 \%$ & $2.0 \%$ & $0.7 \%$ & $0.2 \%$ \\
\hline Triple negative & 3619 & $6.8 \%$ & $4.6 \%$ & $2.7 \%$ & $1.6 \%$ & $1.1 \%$ \\
\hline
\end{tabular}

LR: local recurrence; ER: estrogen receptor, PR: progesterone receptor

\section{Percentage of LRs occurring in each year of follow-up}

On a group level (e.g. in clinical studies) it is of interest to know which proportion of LRs occurs in which years of follow-up. In ER-Her2+ and triple negative tumors, $62.4 \%$ and $69.5 \%$ of the total number of events occurred in the first two years, whereas $40 \%$ would be expected when LRs were distributed equally over 5 years of follow-up (100\%/5 years $=20 \%$ per year). In the ER+ subtypes, the number of LRs was more equally distributed over the five years of follow-up (Table 7.5). 
Table $7.5 \quad$ Number of local recurrences as a first event within 5 years that occurred in each year of followup

\begin{tabular}{lcccccc}
\hline & & \multicolumn{5}{c}{ Number of LRs as a first event within 5 years after diagnosis that } \\
& & \multicolumn{5}{c}{ occurred in each year of follow-up } \\
\cline { 3 - 7 } & Total no. of LRs & In 1st year* & In 2nd year & In 3rd year & In 4th year & In 5th year \\
\hline All patients & $874(100 \%)$ & $203(23.2 \%)$ & $238(27.2 \%)$ & $186(21.3 \%)$ & $127(14.5 \%)$ & $120(13.7 \%)$ \\
Approximate subtypes & & & & & & \\
ER+PR+Her2- & $331(100 \%)$ & $39(11.8 \%)$ & $89(26.9 \%)$ & $77(23.3 \%)$ & $65(19.6 \%)$ & $61(18.4 \%)$ \\
ER+PR-Her2- & $79(100 \%)$ & $13(16.5 \%)$ & $23(29.1 \%)$ & $18(22.8 \%)$ & $13(16.5 \%)$ & $12(15.2 \%)$ \\
ER+Her2+ & $66(100 \%)$ & $14(21.2 \%)$ & $18(27.3 \%)$ & $12(18.2 \%)$ & $12(18.2 \%)$ & $10(15.1 \%)$ \\
ER-Her2+ & $77(100 \%)$ & $24(31.2 \%)$ & $24(31.2 \%)$ & $19(24.7 \%)$ & $7(9.1 \%)$ & $3(3.9 \%)$ \\
Triple negative & $203(100 \%)$ & $81(39.9 \%)$ & $60(29.6 \%)$ & $31(15.3 \%)$ & $14(6.9 \%)$ & $17(8.4 \%)$ \\
\hline
\end{tabular}

* in 1st year: events within 3 months after initial diagnosis were counted as synchronous to the original tumor, thus, 1st year equals 3 months -1 year after diagnosis. LR: local recurrence, ER: estrogen receptor, PR: progesterone receptor

\section{Discussion}

This population-based study of 34.453 breast cancer patients diagnosed between 2005 and 2008 showed that the risk of LR as a first event within 5 years after diagnosis was $3.0 \%$. This risk differed significantly between subtypes, with triple negative tumors being at highest risk with $6.8 \%$ and $E R+P R+H e r 2-$ at the lowest with $2.2 \%$. The difference (ER+PR+Her2-compared to the other types) remained significant when corrected for age, T-status, N-status, grade, type of breast surgery, radiation therapy, chemotherapy, endocrine therapy, and trastuzumab (except ER+PR+Her2- compared to ER+PR-Her2-). With increasing number of event-free years, the risk of having a LR before the end of regular 5-year follow-up decreased. After three event-free years, the risk was $1.0 \%$ or less in all subtypes except triple negative breast cancer (1.6\%). The decrease in the first four years after diagnosis was most pronounced in the higher risk subtypes, namely triple negative (6.8\% to $1.1 \%)$ and ER-Her $2+(4.7 \%$ to $0.2 \%)$ tumors.

In clinical practice, this means that a breast cancer patient who has been event-free for 3 years, has a risk of $1 \%$ or less developing LR as a first event before the end of regular 5 year follow-up (unless triple negative, than 1.6\%). In a research setting (for instance, in a study using LR as an endpoint) for every 100 event-free patients after 3 years of followup, 1 LR can be expected if follow-up is continued until 5 years. This suggests that although recurrences do occur later in follow-up, 3-year results may produce similar results to 5 years, depending on the size of the study.

Our results are in line with publications on breast cancer survival and other cancers, suggesting that improvement with event-free years is greatest for tumors with the worst baseline prognosis. ${ }^{8-11}$ The results reflect that ER- (particularly triple negative) tumors show relatively many early LRs (within 2 years), whereas ER+ tumors have a fairly 
constant rate of LRs throughout the 5 years of follow-up. A study investigating conditional disease-free survival in relation to subtype also showed that ER- tumors conditional DFS improved but suggested that conditional survival decreased for ER+ tumors. This study was limited by a very small number of patients at risk after more than three disease-free years. ${ }^{17}$

The strength of this approach is the large, nationwide and comprehensive database, which includes substantial numbers of patients, even of the less common subtypes. Further, this study provides specific percentages of the chance of LR after a number of event-free years. Although the information on conditional LR can be partly deduced from the slope of the Kaplan-Meier curve, these exact percentages help using the information on the declining risk for determining the use of continued follow-up, both in clinical practice and breast cancer research. Limitations of this study are the lack of follow-up beyond 5 years, which would have been useful especially for ER+ tumors, in which late recurrences are known to occur. ${ }^{18}$ Further, in a population that was treated according to a guideline, bias by indication will occur. This is partially overcome by multivariable analysis. Furthermore, bias by indication is less important in this project compared to other studies, as determining exact estimates of the hazard ratios for treatment and tumor characteristics was not an objective of this study. Due to the inclusion period, tumors were classified according to the $6^{\text {th }}$ edition of the AJCC TNM classification. This is, in terms of primary tumor and local recurrence, the same as the current $7^{\text {th }}$ edition. ${ }^{14}$ In this study, no distinction was made between "true recurrences" and ipsilateral second primary breast cancers, both were counted as local events (consistent with an earlier consensus project ${ }^{16}$ ). This may lead to a higher estimate of LR when compared to studies that do make this distinction.

These results may be used as a starting point for tailoring follow-up to individual needs, both in clinical practice and for breast cancer research. First, a patient who has been event-free for 3 years may ask about the benefit of continued follow-up visits with physical examination and/or mammography to detect LR. Follow-up visits may have different goals beside detecting local recurrence, including monitoring endocrine therapy, encouraging its use, monitoring and treating other side effects of breast cancer treatment, evaluation of psychosocial concerns, and patient reassurance. However, for some patients, a less than $1 \%$ chance of finding a LR may be a reason to discontinue follow-up or tailor it to individual needs. National guidelines may use this information to allow personalized decisions about the duration of follow-up. Different guidelines propose slightly different but similar recommendations for follow-up frequency in the first 5 years, and also differ in their recommendations after 5 years (return to screening 
program, continued annual mammograms, no recommendations). ${ }^{4,5,7,20}$ Of these guidelines, only the ASCO guideline recommends to consider patient preferences and personal risk, based on age, specific diagnosis, and treatment protocol. None of these guidelines describe which specific patient and tumor characteristics should prompt higher or lower frequency or duration of follow-up. Data on conditional local recurrence in relation to subtype may be used as a starting point for tailoring follow-up to individual patients. An even more personalized risk might be calculated with a nomogram, such as proposed by Witteveen et al. ${ }^{19}$, partly on the same population. This model, however, does not incorporate the effect of trastuzumab. Additionally, for breast cancer research using LR as an endpoint, the information on the pattern of LR may be used to determine optimal follow-up time for clinical studies.

In conclusion, in this nationwide database including 34.453 breast cancer patients diagnosed between 2005-2008, the incidence of LR as a first event within 5 years was low overall with $3.0 \%$. The incidence was different between subtypes of breast cancer, $\mathrm{ER}+\mathrm{PR}+\mathrm{Her} 2$ - tumors posed the lowest risk and triple negative tumors the highest. The risk of developing a LR within 5 years of diagnosis decreased with event-free years. After 3 years, this risk was $1 \%$ or less in all subtypes except triple negative cancers. This improvement in prognosis is reassuring to patients during follow-up. It also suggests that follow-up beyond 3 years may have limited yield when it comes to finding additional LR, both for individual patients and clinical studies using LR as the primary outcome. Although there are many reasons to choose longer follow-up, this may be a starting point to tailor follow-up duration to individual needs and preferences. 


\section{References}

1 Allemani C, Minicozzi P, Berrino F, Bastiaannet E, Gavin A, Galceran J, et al. Predictions of survival up to 10 years after diagnosis for European women with breast cancer in 2000-2002. International journal of cancer Journal international du cancer. 2013; 132: 2404-2412.

2 Arrington AK, Goldstein L, Kruper L, Vito C, Yim J, Chen SL. Life expectancy after curative-intent treatment of breast cancer: impact on long-term follow-up care. The American surgeon. 2014; 80: 604-609.

3 Janssen-Heijnen ML, van Steenbergen LN, Voogd AC, Tjan-Heijnen VC, Nijhuis PH, Poortmans PM, et al. Small but significant excess mortality compared with the general population for long-term survivors of breast cancer in the Netherlands. Annals of oncology : official journal of the European Society for Medical Oncology / ESMO. 2014; 25: 64-68.

4 Senkus E, Kyriakides S, Ohno S, Penault-Llorca F, Poortmans P, Rutgers E, et al. Primary breast cancer: ESMO Clinical Practice Guidelines for diagnosis, treatment and follow-up. Annals of oncology : official journal of the European Society for Medical Oncology / ESMO. 2015. pp. v8-v30.

5 Runowicz CD, Leach CR, Henry NL, Henry KS, Mackey HT, Cowens-Alvarado RL, et al. American Cancer Society/American Society of Clinical Oncology Breast Cancer Survivorship Care Guideline. Journal of clinical oncology : official journal of the American Society of Clinical Oncology. 2016. pp. 611-635.

$6 \quad$ NABON, Vereniging van Integrale Kankercentra. Breast Cancer: Nation-wide guideline (2.0). 2012;: 1-297.

7 NICE. Early and locally advanced breast cancer: diagnosis and treatment. Full guideline. National Collaborating Centre for Cancer, editor. Velindre NHS Trust, Cardiff, Wales; 2009.

8 Yu XQ, Baade PD, O'Connell DL. Conditional survival of cancer patients: an Australian perspective. BMC cancer. 2012; 12: 460.

9 Zamboni BA, Yothers G, Choi M, Fuller CD, Dignam JJ, Raich, P. C., et al. Conditional survival and the choice of conditioning set for patients with colon cancer: an analysis of NSABP trials C-03 through C-07. Journal of clinical oncology : official journal of the American Society of Clinical Oncology. 2010;28: 25442548.

10 Janssen-Heijnen ML, Gondos A, Bray F, Hakulinen T, Brewster DH, Brenner H, et al. Clinical relevance of conditional survival of cancer patients in europe: age-specific analyses of 13 cancers. Journal of clinical oncology : official journal of the American Society of Clinical Oncology. 2010; 28: 2520-2528.

11 Kurta ML, Edwards RP, Moysich KB, McDonough K, Bertolet M, Weissfeld JL, et al. Prognosis and conditional disease-free survival among patients with ovarian cancer. Journal of clinical oncology : official journal of the American Society of Clinical Oncology. 2014; 32: 4102-4112.

12 Metzger-Filho O, Sun Z, Viale G, Price KN, Crivellari D, Snyder RD, et al. Patterns of Recurrence and outcome according to breast cancer subtypes in lymph node-negative disease: results from international breast cancer study group trials VIII and IX. Journal of clinical oncology 2013; 31: 3083-3090.

13 World Health Organization. International Classification of Diseases for Oncology, Third Edition [Internet]. 3rd ed. Geneva; 2000 [cited 2017 May 16]. Available from: http://codes.iarc.fr/codegroup/1

14 AJCC AJCOC. AJCC 6th Ed Cancer Staging Manual [Internet]. httpscancerstaging.orgreferencestoolsdeskreferencesPagesdefault.aspx. 2000 [cited 2017 May 16]. Available from: https://cancerstaging.org/references-tools/deskreferences/Pages/default.aspx

15 CBO Kwaliteitsinstituut voor de Gezondheidszorg, Vereniging van Integrale Kankercentra. Guideline 'Treatment of breast cancer' (Richtlijn 'Behandeling van het Mammacarcinoom') [Internet]. Kwaliteitsinstituut voor de Gezondheidszorg CBO; Vereniging van Integrale Kankercentra; National Borstkanker Overleg NABON; 2005. Available from: http://www.oncoline.nl/uploaded/FILES/ mammacarcinoom/Richtlijn\%20Behandeling\%20van\%20het\%20Mammacarcinoom\%20oktober\%202005 .pdf

16 Moossdorff M, van Roozendaal LM, Strobbe LJ, Aebi S, Cameron DA, Dixon JM, et al. Maastricht Delphi Consensus on Event Definitions for Classification of Recurrence in Breast Cancer Research. Journal of the National Cancer Institute. 2014; 106: dju288-dju288.

17 Paik H-J, Lee SK, Ryu JM, Park S, Kim I, Bae SY, et al. Conditional disease-free survival among patients with breast cancer. Medicine (Baltimore). 2017; 96: e5746. 
18 Ignatiadis M, Sotiriou C. Luminal breast cancer: from biology to treatment. Nat Rev Clin Oncol. 2013;10: 494-506.

19 Witteveen A, Vliegen IMH, Sonke GS, Klaase JM, IJzerman MJ, Siesling S. Personalisation of breast cancer follow-up: a time-dependent prognostic nomogram for the estimation of annual risk of locoregional recurrence in early breast cancer patients. Breast Cancer Res Treat. 2015;152:627-636.

20 NCCN. National Comprehensive Cancer Network - NCCN Guidelines Breast Cancer Version 2.2017 [Internet]. Gradishar WJ, editor. 2017 [cited 2017 May 19]. Available from: https://www.nccn.org/ professionals/physician_gls/pdf/breast_blocks.pdf 



\title{
Chapter 8
}

\section{Conditional regional recurrence for different subtypes}

\author{
of breast cancer
}

Vane M, Moossdorff M, Maaren MC van, Kuijk SMJ van, Nijnatten TJA van, Goorts B, Roozendaal LM van, Boerma EG, Wilt JHW de, Smidt ML Submitted 


\section{Abstract}

\section{Background}

Regional recurrence (RR) is an endpoint in several trials concerning reducing axillary treatment in CT1-2NO breast cancer patients. Topic of debate regarding these trials is adequate follow-up time. The risk of RR may decrease with each subsequent event-free year, affecting the yield and consequently usefulness of long(er) follow-up. The aim of this study is to determine the risk of RR as a first event within 5 years after diagnosis in five subtypes of breast cancer, conditional to being event-free for 1, 2, 3 and 4 years.

\section{Methods}

From the Netherlands Cancer Registry, cT1-2NO breast cancer patients diagnosed from 2005 to 2008 were analyzed. Subgroup analysis was performed for pT1-2N+(sn) patients. RR risk was calculated with Kaplan-Meier analysis. Conditional RR (assuming $x$ event-free years) was determined by selecting patients without an event at $x$ years, and calculating the remaining risk for RR within 5 years after diagnosis.

\section{Results}

A total of 18,009 cT1-2NO (all pN stages) breast cancer patients were included. RR occurred in $1.3 \%$ in cT1-2NO and $1.5 \%$ in $\mathrm{pT} 1-2 \mathrm{~N}+(\mathrm{sn})$ patients. The risk of RR varied between subtypes; it was highest for triple negative tumors and lowest for ER+PR+Her2and ER+HER2+ tumors. After 1, 2, 3, and 4 event-free years, the risk of RR decreased in both groups and in all subtypes. After 2 event-free years, the risk of RR is $0.8 \%$.

\section{Conclusions}

The absolute yield of follow-up beyond two years concerning RR is low; for every 125 event-free patients, one RR can be expected until 5 years. This suggests that followup longer than two years is of limited value for detecting RR in both clinical and research setting. 


\section{Introduction}

As a result of several recent (e.g. ACOSOG Z0011, IBCSG 23-01 and AMAROS)and ongoing (e.g. BOOG 2013-07, POSNOC, SENOMAC, SINODAR, BOOG 2013-08, SOUND, INSEMA, and NCT01821768) randomized controlled trials, the extent of axillary treatment in breast cancer patients is being reduced. ${ }^{1-6}$ Frequently used endpoints in these trials are regional recurrence (RR), disease-free survival (DFS) and overall survival (OS). These endpoints are standardly reported as rates after 5 and 10-years of follow-up. However, these rates are likely to improve in case a patient remains event-free for several years.

Conditional survival is defined as the probability of surviving an additional $\mathrm{x}$ years given that a patient has already survived a number of years after diagnosis. ${ }^{7}$ Previous studies assessed conditional OS and DFS among breast cancer patients. ${ }^{8-11}$ These studies showed that conditional survival improves over time, in particular among patients with the worst prognosis at baseline (e.g. stage III versus stage I-II). ${ }^{11}$

This is in accordance to ovarian, colorectal, endometrial, and testicular cancer and melanoma patients, in which prognosis for cancer survivors generally improves with each event-free year. ${ }^{10,12,13}$ No prior studies have assessed conditional RR among breast cancer patients. It is conceivable that in line with OS and DFS the risk for RR might decrease after event-free years.

Adequate duration of follow-up in both clinical and research setting remains controversial. Most studies report their first results after 5 years, but it has been suggested that most RRs occur in the first years after diagnosis. This questions the yield and therefore use of longer follow-up for this purpose. Another topic of debate in these randomized controlled trials is whether different subtypes of breast cancer might require a different approach. The benefit of computing an individual's RR rate is gaining more tailored prognostic information and follow-up time for breast cancer survivors.

The aim of this study is to determine the risk of RR as a first event within 5 years after diagnosis, conditional to being event-free for $1,2,3$, and 4 years. This study will focus on clinically node negative breast cancer patients in general, and additionally on patients with sentinel node involvement. Conditional RR will be presented separately for ER+PR+Her2-, ER+PR-Her2-, ER+Her2+, ER-Her2+, and triple negative tumors. 


\section{Methods}

\section{Data collection}

The Netherlands Cancer Registry (NCR) data is based on all new breast cancer patients from all Dutch hospitals. Data on patient and tumor characteristics, surgical, radiation and systemic treatment were routinely retrieved from patients' records by trained data registrars of the Netherlands Comprehensive Cancer Organisation (IKNL).

For patients diagnosed between 2005 and 2008, an active follow-up was conducted in which data on first breast cancer event within 5 years after diagnosis was gathered directly from patient files. First breast cancer event was registered as new primary ipsilateral breast cancer, contralateral breast cancer, local recurrence, regional recurrence or distant recurrence.

\section{Study population}

We analyzed the risk of RR in women between 2005 and 2008 diagnosed with primary invasive breast cancer in the Netherlands. This study focused on the study populations of previous mentioned randomized controlled trials, involving breast cancer patients with a clinically T1-2 tumor and clinically node negative status. First, the overall clinically T1-2NO population (consistent with the study population of BOOG 2013-08, SOUND, INSEMA and NCT01821768) was analyzed. ${ }^{6}$ Second, patients from this population with a positive sentinel lymph node (SLN) (consistent with the study population of ACOSOG Z0011, IBCSG 23-01, AMAROS, BOOG 2013-07, POSNOC, SENOMAC and SINODAR) were analyzed separately. ${ }^{3-6}$ These patients will be further referred to as the pT1-2N+(sn) subpopulation. Patients were excluded in case of distant metastasis at (or within 91 days of) diagnosis, an incomplete 5 year follow-up, treatment with primary systemic therapy, or in case of no sentinel lymph node biopsy (SLNB) or incomplete registered results.

\section{Locoregional treatment}

Patients were treated according to the Dutch breast cancer guidelines of $2005 .{ }^{14}$ All patients had clinically T1-2 tumors and were clinically node negative (based on physical examination, axillary ultrasound was common but not mandatory).

Locoregional treatment consisted of breast conserving therapy (lumpectomy and whole breast radiotherapy) or mastectomy combined with an SLNB. Patients with a positive SLN were treated with an axillary lymph node dissection (ALND) or axillary radiotherapy, in context of the AMAROS trial. 


\section{Systemic treatment}

Adjuvant systemic treatment was recommended for all pN+ breast cancer patients. Adjuvant systemic treatment for NO patients was recommended for patients $<35$ years and for patients $\geq 35$ years with risk factors. Risk factors were tumor $\geq 3 \mathrm{~cm}$, or tumor $\geq 1 \mathrm{~cm}$ and grade $\mathrm{II}$, or tumor $\geq 2 \mathrm{~cm}$ and grade $\mathrm{II}$. Chemotherapy regimen consisted of five courses 5 Fluorouracil, Epirubicin, Cyclophosphamide (FEC) or six courses of Taxotere, Adriamycin and Cyclophosphamide (TAC). Endocrine therapy (Tamoxifen and/or Luteinizing hormone-releasing hormone agonist) was recommended for ER+ and/or PR+ tumours. In case of Her2Neu receptor (HER 2) amplification, targeted therapy (trastuzumab) was given in addition to chemotherapy.

\section{Endpoints}

The primary endpoint was conditional RR, defined as the risk of RR as a first event within 5 years after diagnosis, conditional to being event-free for 1, 2, 3, and 4 years. RR included recurrence in an ipsilateral axillary-, infraclavicular-, or supraclavicular lymph node, internal mammary/parasternal or intramammary lymph node. ${ }^{15}$

RR within 91 days following diagnosis was regarded as a synchronous event and excluded from analysis. Patients were censored at the date of their first event, at the date of last follow-up, or at the date of death. If another event occurred within 91 days of the first recurrence, this was considered synchronous to the first event, and also counted as a first recurrence.

\section{Statistical analysis}

Statistical analyses were performed using the Statistical Package for the Social Sciences (SPSS), version 22.0 (IBM Corporation, Armonk, NY, USA). RR was determined for the overall population and for the subgroup of clinically node negative patients with positive lymph nodes. Kaplan-Meier analysis was used to determine the probability of RR over time. Significance of the difference between the subtypes (ER+PR+Her2-, ER+PR-Her2-, ER+Her2+, ER-Her2+, and ER-PR-Her2-) was tested with the log-rank test. Multivariable Cox regression was used to determine the effect of subtype corrected for several prognostic variables that may differ among the groups. The risk of conditional RR was calculated by selecting patients who were event free (i.e. no local recurrence, RR, distant recurrence, second primary breast cancer, or death) at 1, 2, 3 and 4 years. The risk of RR within 5 years of diagnosis was calculated for each time point and for five approximate subtypes of breast cancer. A p-value $\leq 0.05$ was considered as statistically significant. 


\section{Results}

\section{Patient demographics and primary tumor characteristics}

A total of 18,009 primary clinically T1-2N0 breast cancer patients were included. Patient and tumor characteristics are summarized in Table 8.1. Median age was 59 years (range 22-98). The most prevalent subtype was ER+PR+Her2- in 9,929 patients (55.1\%), followed by ER+PR-Her2- in 2,032 patients (11.3\%), triple negative tumors in 1,701 patients (9.5\%), ER+Her2+ in 1,231 patients (6.8\%) and ER-Her2+ in 667 patients (3.7\%). Subtype was unknown in 2,449 of the patients (13.6\%). All patients underwent an SLNB for determining axillary lymph node status. Patient and tumor characteristics per subtype are shown in Appendix 8.1.

Table 8.1 Patient demographics and tumor characteristics of the cT1-2N0 population $(\mathrm{N}=18,009)$

\begin{tabular}{|c|c|c|c|}
\hline $\begin{array}{l}\text { Age, years } \\
\text { Median } \\
\text { range }\end{array}$ & $\begin{array}{c}59 \\
22-98 \\
\end{array}$ & $\begin{array}{l}\text { Surgical treatment, } \mathrm{n}(\%) \\
\text { breast conserving } \\
\text { mastectomy }\end{array}$ & $\begin{array}{r}12173(67.6) \\
5836(32.4) \\
\end{array}$ \\
\hline $\begin{array}{l}\text { Tumor type, n (\%) } \\
\text { ductal } \\
\text { lobular } \\
\text { mixed or other }\end{array}$ & $\begin{array}{l}13640(75.7) \\
1858(10.3) \\
2511(14.0)\end{array}$ & $\begin{array}{l}\text { pN-stadium, n (\%) } \\
\text { pN0 } \\
\text { pN1mi } \\
\text { pN1a } \\
\text { pN1b } \\
\text { pN2 } \\
\text { pN3 } \\
\text { unknown }\end{array}$ & $\begin{array}{c}13177(73.2) \\
1211(6.7) \\
2813(15.6) \\
29(0.1) \\
519(2.9) \\
177(1.0) \\
36(0.2) \\
\end{array}$ \\
\hline $\begin{array}{l}\text { Grade (Bloom-Richardson), n (\%) } \\
\text { I } \\
\text { II } \\
\text { III } \\
\text { unknown }\end{array}$ & $\begin{array}{l}4730(26.3) \\
7774(43.2) \\
4872(27.0) \\
663(3.5) \\
\end{array}$ & $\begin{array}{l}\text { Chemotherapy, n (\%) } \\
\text { yes } \\
\text { no }\end{array}$ & $\begin{array}{r}5767(32.0) \\
12242(68.0)\end{array}$ \\
\hline $\begin{array}{l}\text { Subtypes, } n(\%) \\
\text { ER+PR+Her2- } \\
\text { ER+PR-Her2- } \\
\text { ER+Her2+ } \\
\text { ER-Her2+ } \\
\text { triple negative } \\
\text { unknown } \\
\end{array}$ & $\begin{array}{c}9929(55.1) \\
2032(11.3) \\
1231(6.8) \\
667(3.7) \\
1701(9.5) \\
2449(13.6) \\
\end{array}$ & $\begin{array}{l}\text { Hormone therapy for } \mathrm{ER}+, \mathrm{n}(\%) \\
\text { yes } \\
\text { no }\end{array}$ & $\begin{array}{l}7102(47.2) \\
7935(52.8)\end{array}$ \\
\hline $\begin{array}{l}\text { cT-stadium, n (\%) } \\
\text { cT1 } \\
\text { cT2 }\end{array}$ & $\begin{array}{r}13809(76.7) \\
4200(23.3)\end{array}$ & $\begin{array}{l}\text { Trastuzumab and chemotherapy for } \\
\text { HER2+, n (\%) } \\
\text { yes } \\
\text { no }\end{array}$ & $\begin{array}{l}933(49.3) \\
974(50.7)\end{array}$ \\
\hline $\begin{array}{l}\text { pT-stadium, n (\%) } \\
\text { pT0 } \\
\text { pT1 } \\
\text { pT2 } \\
\text { pT3 } \\
\text { pT4 } \\
\text { unknown }\end{array}$ & $\begin{array}{c}1(0.0) \\
12332(68.5) \\
5422(30.1) \\
157(0.9) \\
18(0.1) \\
79(0.4)\end{array}$ & & \\
\hline
\end{tabular}

$\mathrm{N}$ number of cases, ER estrogen receptor, PR progesterone receptor, HER2 human epidermal growth factor receptor 2, cT clinical tumor stadium, pT pathological tumor stadium. 


\section{The effect of event-free years on risk of regional recurrence within}

\section{5 years}

Median follow-up time was 58.3 months (range 0.07-60.02). The incidence of RR as a first event within 5 years of diagnosis was 1.3\% in the overall cT1-2NO group, and $1.5 \%$ in the subpopulation of $\mathrm{pT} 1-2 \mathrm{~N}+(\mathrm{sn})$ patients. These results were corrected for confounders, for both the overall cT1-2N0 group and subpopulation of pT1-2N+(sn) (Appendix 8.2). After 1, 2, 3 and 4 event-free years, the risk of developing RR in the remaining period decreased in both groups. In the overall cT1-2NO group, the risk of RR decreased with additional event-free years to $1.1 \%, 0.8 \%, 0.6 \%$, and $0.3 \%$, respectively (Table 8.2). In the pT1-2N+(sn) subpopulation, the risk of RR decreased to $1.2 \%, 0.8 \%$, $0.6 \%$, and $0.4 \%$, respectively (Table 8.3 ). In both the overall cT1-2N0 group and in the pT1-2N+(sn) subpopulation, the risk of RR as a first event, after 2 event-free years was $0.8 \%$.

Table 8.2 Impact of a number of event-free years on the risk of RR as a first event within 5 years after diagnosis in clinically node negative patients (cT1-2N0)

\begin{tabular}{|c|c|c|c|c|c|c|}
\hline & \multirow[b]{2}{*}{$\mathrm{N}$} & \multirow[b]{2}{*}{$\begin{array}{l}\text { Risk of 5-year } \\
\text { RR at diagnosis }\end{array}$} & \multicolumn{4}{|c|}{$\begin{array}{l}\text { Risk of regional recurrence within } 5 \text { years after diagnosis, } \\
\text { after } x \text { event-free years }\end{array}$} \\
\hline & & & $\begin{array}{l}\text { After } 1 \text { event- } \\
\text { free year }\end{array}$ & $\begin{array}{l}\text { After } 2 \text { event- } \\
\text { free years }\end{array}$ & $\begin{array}{c}\text { After } 3 \text { event- } \\
\text { free years }\end{array}$ & $\begin{array}{l}\text { After } 4 \text { event- } \\
\text { free years }\end{array}$ \\
\hline All patients & 18009 & $\begin{array}{c}1.3 \% \\
(206 / 18009) \\
\end{array}$ & $\begin{array}{c}1.1 \% \\
(163 / 17460) \\
\end{array}$ & $\begin{array}{c}0.8 \% \\
(117 / 16693) \\
\end{array}$ & $\begin{array}{c}0.6 \% \\
(77 / 15891) \\
\end{array}$ & $\begin{array}{c}0.3 \% \\
(35 / 14749) \\
\end{array}$ \\
\hline \multicolumn{7}{|c|}{ Breast cancer subtypes } \\
\hline ER+PR+Her2- & 9929 & $\begin{array}{c}0.8 \% \\
(67 / 9929)\end{array}$ & $\begin{array}{c}0.8 \% \\
(61 / 9695)\end{array}$ & $\begin{array}{c}0.7 \% \\
(51 / 9346)\end{array}$ & $\begin{array}{c}0.5 \% \\
(10 / 3151)\end{array}$ & $\begin{array}{c}0.2 \% \\
(16 / 8316)\end{array}$ \\
\hline ER+PR-Her2- & 2032 & $\begin{array}{c}1.5 \% \\
(27 / 2032)\end{array}$ & $\begin{array}{c}1.2 \% \\
(21 / 1958)\end{array}$ & $\begin{array}{c}0.9 \% \\
(15 / 1873)\end{array}$ & $\begin{array}{c}0.5 \% \\
(3 / 568)\end{array}$ & $\begin{array}{c}0.3 \% \\
(4 / 1644)\end{array}$ \\
\hline ER+Her2+ & 1231 & $\begin{array}{c}1.4 \% \\
(15 / 1231)\end{array}$ & $\begin{array}{c}1.3 \% \\
(14 / 1204)\end{array}$ & $\begin{array}{c}1.1 \% \\
(11 / 1155)\end{array}$ & $\begin{array}{c}0.7 \% \\
(7 / 1098)\end{array}$ & $\begin{array}{c}0.3 \% \\
(2 / 1031)\end{array}$ \\
\hline ER-Her2+ & 667 & $\begin{array}{c}1.8 \% \\
(11 / 667)\end{array}$ & $\begin{array}{c}1.3 \% \\
(8 / 641)\end{array}$ & $\begin{array}{c}0.7 \% \\
(4 / 601)\end{array}$ & $\begin{array}{c}0.6 \% \\
(3 / 568)\end{array}$ & $\begin{array}{c}0.2 \% \\
(1 / 525)\end{array}$ \\
\hline Triple negative & 1701 & $\begin{array}{c}3.7 \% \\
(54 / 1701)\end{array}$ & $\begin{array}{c}2.6 \% \\
(36 / 1594)\end{array}$ & $\begin{array}{c}1.4 \% \\
(17 / 1449)\end{array}$ & $\begin{array}{c}0.9 \% \\
(10 / 1351)\end{array}$ & $\begin{array}{c}0.4 \% \\
(3 / 1255)\end{array}$ \\
\hline
\end{tabular}

\section{Regional recurrence as a first event between different subtypes}

The risk of RR at diagnosis in the overall cT1-2N0 group varied between subtypes, and was highest for triple negative (3.7\%) and lowest for ER+PR+Her2- tumors (0.8\%) (Table 8.2). The difference between the subtypes ER+PR+Her2- and ER+PR-Her2- $(0.8 \%$ vs. $1.5 \%, p=0.001)$; and between ER-Her2+ and triple negative were significant ( $1.8 \%$ vs. $3.7 \%, p=0.029$ ) (Figure 8.1). In the subpopulation of $\mathrm{pT} 1-2 \mathrm{~N}+(\mathrm{sn})$, the risk of $\mathrm{RR}$ at diagnosis also varied between subtypes, and was highest for triple negative $(10.7 \%)$ and lowest for ER+Her2+ tumors (0.4\%) and ER+PR+Her2- (0.5\%) (Table 8.3). The difference 
between the subtypes in the $\mathrm{pT} 1-2 \mathrm{~N}+(\mathrm{sn})$ subpopulation were significant in $\mathrm{ER}+\mathrm{PR}+\mathrm{Her} 2-$ and ER+PR-Her- (0.5\% vs. $1.9 \% \mathrm{p}=0.011), \mathrm{ER}+\mathrm{PR}-\mathrm{Her}-$ and ER+Her2+ $(1.9 \%$ vs. $0.4 \%, p=0.077), E R+H e r 2+$ and ER-Her2+ (0.4\% vs. $3.4 \%, p=0.006)$ and ER-Her2+ and triple negative (3.4\% vs. 10.7\%, $p=0.015$ ) (Figure 8.2 ).

Table 8.3 Impact of a number of event-free years on the risk of RR as a first event within 5 years after diagnosis in clinically node negative patients with a positive SLN (pT1-2N+(sn))

\begin{tabular}{|c|c|c|c|c|c|c|}
\hline & \multirow[b]{2}{*}{$\mathrm{N}$} & \multirow[b]{2}{*}{$\begin{array}{l}\text { Risk of } 5 \text {-year } \\
\text { RR at diagnosis }\end{array}$} & \multicolumn{4}{|c|}{$\begin{array}{l}\text { Risk of regional recurrence within } 5 \text { years after diagnosis, after } \mathrm{x} \\
\text { event-free years }\end{array}$} \\
\hline & & & $\begin{array}{l}\text { After } 1 \text { event- } \\
\text { free year }\end{array}$ & $\begin{array}{l}\text { After } 2 \text { event- } \\
\text { free years }\end{array}$ & $\begin{array}{c}\text { After } 3 \\
\text { event-free } \\
\text { years }\end{array}$ & $\begin{array}{c}\text { After } 4 \\
\text { event-free years }\end{array}$ \\
\hline All patients & 4348 & $\begin{array}{c}1.5 \% \\
(58 / 4348)\end{array}$ & $\begin{array}{c}1.2 \% \\
(45 / 4194)\end{array}$ & $\begin{array}{c}0.8 \% \\
(27 / 4002)\end{array}$ & $\begin{array}{c}0.6 \% \\
(19 / 3798)\end{array}$ & $\begin{array}{c}0.4 \% \\
(12 / 3559)\end{array}$ \\
\hline $\mathrm{ER}+\mathrm{PR}+\mathrm{Her} 2-$ & 2630 & $\begin{array}{c}0.5 \% \\
(13 / 2630)\end{array}$ & $\begin{array}{c}0.4 \% \\
(9 / 2558)\end{array}$ & $\begin{array}{c}0.3 \% \\
(7 / 2472)\end{array}$ & $\begin{array}{c}0.2 \\
(5 / 2372)\end{array}$ & $\begin{array}{c}0.2 \% \\
(4 / 2244)\end{array}$ \\
\hline ER+PR-Her2- & 480 & $\begin{array}{c}1.9 \% \\
(7 / 480)\end{array}$ & $\begin{array}{c}1.5 \% \\
(5 / 457)\end{array}$ & $\begin{array}{c}1.0 \% \\
(3 / 438)\end{array}$ & $\begin{array}{c}0.8 \% \\
(2 / 406)\end{array}$ & $\begin{array}{c}0.8 \% \\
(2 / 371)\end{array}$ \\
\hline $\mathrm{ER}+\mathrm{Her} 2+$ & 366 & $\begin{array}{c}0.4 \% \\
(1 / 366)\end{array}$ & $\begin{array}{c}0.4 \% \\
(1 / 328)\end{array}$ & $\begin{array}{c}0.4 \% \\
(1 / 312)\end{array}$ & $\begin{array}{c}0.4 \% \\
(1 / 298)\end{array}$ & $\begin{array}{c}0.4 \% \\
(1 / 279)\end{array}$ \\
\hline ER-Her2+ & 336 & $\begin{array}{c}3.4 \% \\
(5 / 157)\end{array}$ & $\begin{array}{c}3.4 \% \\
(5 / 152)\end{array}$ & $\begin{array}{c}1.5 \% \\
(2 / 143)\end{array}$ & $\begin{array}{c}1.5 \% \\
(2 / 137)\end{array}$ & $\begin{array}{c}0.0 \% \\
(0 / 126)\end{array}$ \\
\hline Triple negative & 293 & $\begin{array}{c}10.7 \% \\
(24 / 293)\end{array}$ & $\begin{array}{c}8.7 \% \\
(18 / 257)\end{array}$ & $\begin{array}{c}5.2 \% \\
(9 / 220)\end{array}$ & $\begin{array}{c}2.8 \% \\
(4 / 191)\end{array}$ & $\begin{array}{c}1.2 \% \\
(1 / 173)\end{array}$ \\
\hline
\end{tabular}

\section{The effect of event-free years on risk of regional recurrence between subtypes}

The risk of RR as a first event within 5 years after diagnosis decreased in all subtypes from both the overall and subgroup, when more event-free years had passed. Triple negative tumors had the worst prognosis at baseline, but showed proportionally the largest decrease: $3.7 \%$ to $0.4 \%$ in the cT1-2NO group, and $10.7 \%$ to $1.2 \%$ in the pT1$2 \mathrm{~N}+(\mathrm{sn})$ subgroup. Tumors with the best prognosis at baseline, which were ER+PR+Her2tumors in the overall cT1-2NO group (0.8\% to $0.2 \%)$, and ER+Her2+ tumors (0.4\% to $0.4 \%)$ and ER+PR+Her2- $(0.5 \%$ to $0.2 \%)$ in the $\mathrm{pT} 1-2 \mathrm{~N}+(\mathrm{sn})$ subgroup, showed proportionally the smallest decrease. After 2 event-free years, the overall risk of developing RR within 5 years, was less than $1 \%$ in the cT1-2NO group and pT1-2N+(sn) patients (Table 8.2 and 8.3).

Triple negative tumors in the cT1-2NO group achieved this low rate after 3 event-free years. In the subgroup of pT1-2N+(sn) patients, the risk of developing RR within 5 years was less than $1 \%$ after 3 event-free years was, except for ER-Her2+ (1.5\%) and triple negative tumors (5.2\%) (Table 8.3). 


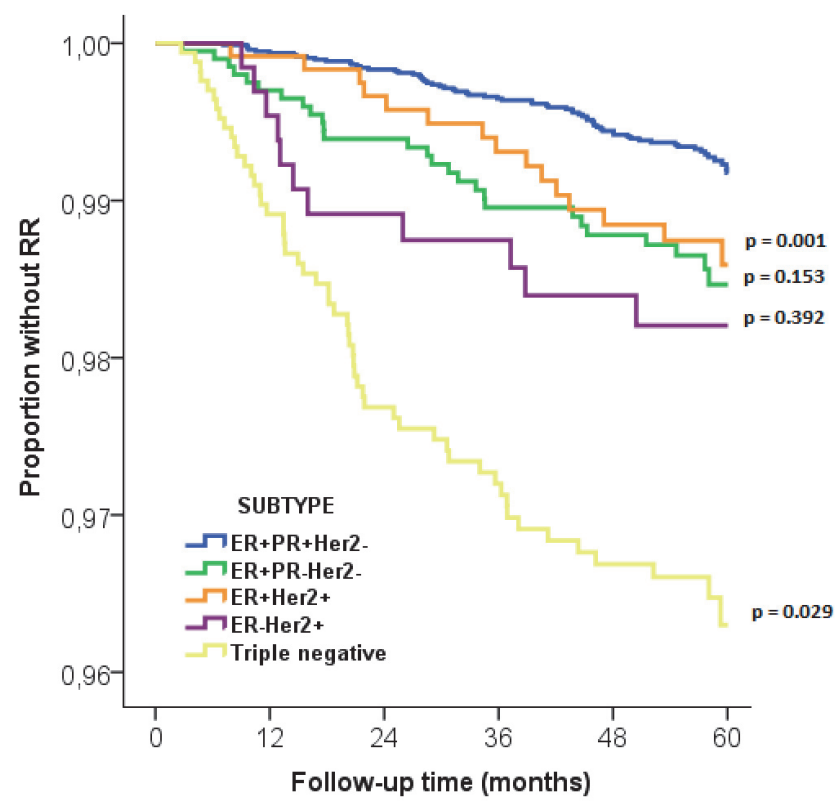

Figure 8.1 Risk of regional recurrence as a first event between different subtypes in cT1-2NO breast cancer

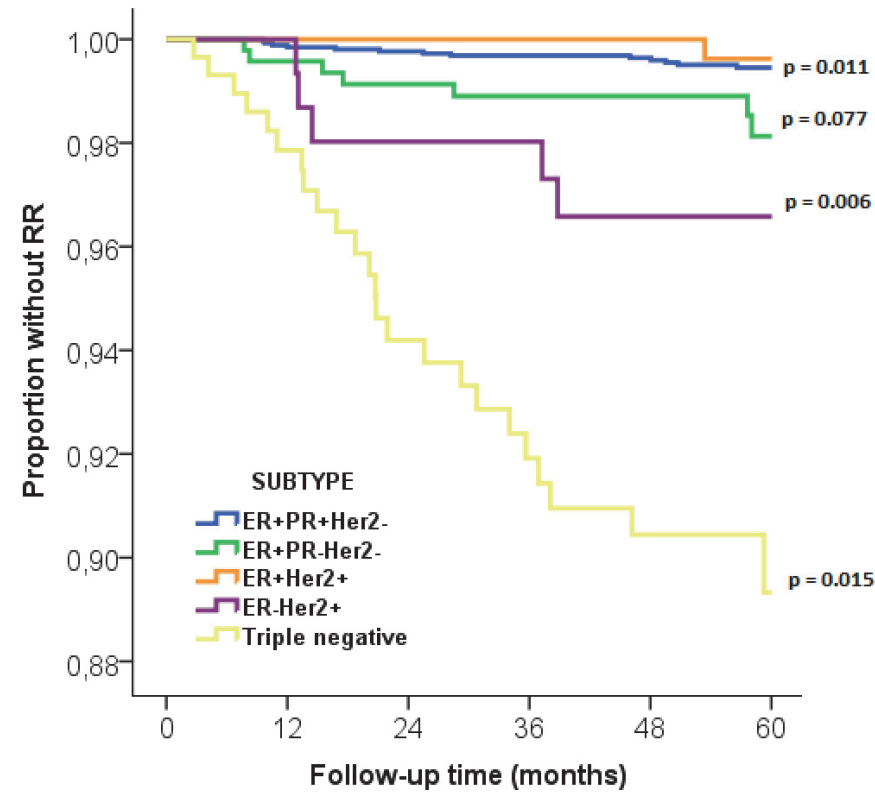

Figure 8.2 Risk of regional recurrence as a first event between different subtypes in pT1-2N+(sn) breast cancer 


\section{Discussion}

The aim of this study was to determine the risk of RR as a first event within 5 years after diagnosis, conditional to being event-free for $1,2,3$, and 4 years. In the overall cT1-2N0 group, the risk of RR was $1.3 \%$, and $1.5 \%$ in the $\mathrm{pT} 1-2 \mathrm{~N}+(\mathrm{sn})$ subpopulation. In the overall group and subpopulation, the risk of RR significantly differed between subtypes. The risk of RR decreased in both groups and in all subtypes when more event-free years passed.

Studies of Allemani et al., Arrington et al. and Janssen-Heijnen et al. showed that conditional DFS and OS improves as time elapses since breast cancer diagnosis. ${ }^{8,9.11}$ Furthermore, the study of Janssen-Heijnen et al. showed a clear difference in conditional survival between stage (favorable for stage III versus stage I-II) and between age groups (favorable for age groups 45-54 and 55-64 years). These differences in conditional survival remained significant, but decreased in time. ${ }^{10,11}$ None of these studies reported the impact of subtype as a prognostic factor on conditional survival. In the current era, subtypes of breast cancer have become more important in addition to traditional prognostic factors, such as age and stage.

The strength of this study is the large cohort of 18,009 breast cancer patients. All new Dutch breast cancer patients diagnosed between 2005 and 2008 were included. Therefore all subtypes, including ER+PR+Her2-, ER+PR-Her2-, ER+Her2+, ER-Her2+, and even triple negative tumors are adequately represented in this cohort. Although triple negative breast cancer patients were less frequently diagnosed with a positive SLN at diagnosis compared to other subtypes, these tumors had the highest risk of RR as a first event within 5 years after diagnosis $3.7 \%$ in the overall group and $10.7 \%$ in the SN positive subpopulation). The systematic review of Lowery et al. concluded that locoregional recurrence was significantly higher in triple negative tumors compared with other subtypes. ${ }^{16}$ Metzger et al. also observed an increased incidence of RR in triple negative tumors compared to other subtypes. ${ }^{17}$ In contrast, van Roozendaal et al. showed that RR occurred in only $2.9 \%$ of the triple negative CT1-2NO patients. ${ }^{18}$ This study showed that the decrease in risk of RR was most explicit in the subtype with the highest risk at baseline (triple negative tumors). This is consistent with previous studies, which suggested that improvement with event-free years is greatest for tumors with the worst prognosis at baseline. ${ }^{11}$

Based on these results, physicians can use conditional RR for more patient tailored prognosis after 1, 2, 3, and 4 event-free years classified by subtype. In clinical setting, 
follow-up is continued to at least 5 years after diagnosis. However, in only one of the 125 patients a RR will occur in the third, fourth, and fifth years of follow-up. This suggests that longer follow-up is of limited value for detection of RR, although this may be required for other outcomes. Furthermore, this study showed that most patients with highest risk of RR at baseline (triple negative $\mathrm{pT} 1-2 \mathrm{~N}+(\mathrm{sn})$ tumors) will develop RR early during follow-up. So even in these tumors, follow-up after three years is of limited value for detection of RR. The information on conditional RR can also be applied in clinical research.

Limitation of this study is the lack of follow-up beyond 5 years. However, Matsen et al. showed that the majority of RR occurred within the first 5 years after surgery. ${ }^{19}$

Late RR defined as RR after more than 5 years of surgery, occurred in only five of the 1,529 included patients. The recently published 10-year results of the ACOSOG Z0011 trial showed that from 5 to 10 years of follow-up, in only two patients a RR occurred in the ALND group versus five in the SLNB alone group. ${ }^{20}$ These results imply that late RR after a negative SLNB is rare. The question remains whether this is also applicable to ER+ tumors treated with at least 5 years of hormone therapy. ${ }^{21,22}$ Further, this analysis includes all patients with a positive SLN, i.e. 1-3 and 4 or more, as only the total number of positive nodes was registered and not the number of positive SLNs. Another limitation of this study is that only the first event (RR) within 5 years after diagnosis was registered, which could have resulted in an underestimated number of events. Finally, patients were treated according to the Dutch breast cancer guideline of 2005 . This differs from current guideline concerning that axillary ultrasound was common but not mandatory and indication changed chemo-, hormone and immunotherapy regimens.

In conclusion, the overall risk of RR as a first event was low (1.3\%). After 1, 2, 3 and 4 event-free years, the risk of RR decreased in both groups and all subtypes. The absolute yield of follow-up beyond two years concerning RR is low (0.8\%); for every 125 eventfree patients, one RR can be expected until 5-years. This suggests that follow-up longer than two years is of limited value for detecting RR in both clinical and research setting. 


\section{References}

1. Giuliano AE, Hunt KK, Ballman KV, Beitsch PD, Whitworth PW, Blumencranz PW, et al. Axillary dissection vs no axillary dissection in women with invasive breast cancer and sentinel node metastasis: a randomized clinical trial. JAMA. 2011;305(6):569-575.

2. Galimberti V, Cole BF, Zurrida S, Viale G, Luini A, Veronesi P, et al. Axillary dissection versus no axillary dissection in patients with sentinel-node micrometastases (IBCSG 23-01): a phase 3 randomised controlled trial. Lancet Oncol. 2013;14(4):297-305.

3. Donker M, Slaets L, van Tienhoven G, Rutgers EJ. [Axillary lymph node dissection versus axillary radiotherapy in patients with a positive sentinel node: the AMAROS trial]. Ned Tijdschr Geneeskd. 2015;159:A9302.

4. van Roozendaal LM, de Wilt JH, van Dalen T, van der Hage JA, Strobbe LJ, Boersma LJ, et al. The value of completion axillary treatment in sentinel node positive breast cancer patients undergoing a mastectomy: a Dutch randomized controlled multicentre trial (BOOG 2013-07). BMC Cancer. 2015;15:610.

5. Goyal A, Dodwell D. POSNOC: A Randomised Trial Looking at Axillary Treatment in Women with One or Two Sentinel Nodes with Macrometastases. Clin Oncol. 2015;27(12):692-695.

6. Gentilini O, Veronesi U. Abandoning sentinel lymph node biopsy in early breast cancer? A new trial in progress at the European Institute of Oncology of Milan (SOUND: Sentinel node vs Observation after axillary UltraSouND). Breast. 2012;21(5):678-861.

7. Henson DE, Ries LA, Carriaga MT. Conditional survival of 56,268 patients with breast cancer. Cancer. 1995; 76(2):237-242.

8. Allemani C, Minicozzi P, Berrino F, Bastiaannet E, Gavin A, Galceran J, et al. Predictions of survival up to 10 years after diagnosis for European women with breast cancer in 2000-2002. Int J Cancer. 2013;132(10):2404-2412.

9. Arrington AK, Goldstein L, Kruper L, Vito C, Yim J, Chen SL. Life expectancy after curative-intent treatment of breast cancer: impact on long-term follow-up care. Am Surg. 2014;80(6):604-609.

10. Janssen-Heijnen ML, Gondos A, Bray F, Hakulinen T, Brewster DH, Brenner H, et al. Clinical relevance of conditional survival of cancer patients in europe: age-specific analyses of 13 cancers. J Clin Oncol. 2010;28(15):2520-2528.

11. Janssen-Heijnen ML, van Steenbergen LN, Voogd AC, Tjan-Heijnen VC, Nijhuis PH, Poortmans PM, et al. Small but significant excess mortality compared with the general population for long-term survivors of breast cancer in the Netherlands. Ann Oncol. 2014;25(1):64-68.

12. Kurta ML, Edwards RP, Moysich KB, McDonough K, Bertolet M, Weissfeld JL, et al. Prognosis and conditional disease-free survival among patients with ovarian cancer. J Clin Oncol. 2014;32(36): 4102-4112.

13. Zamboni BA, Yothers G, Choi M, Fuller CD, Dignam JJ, Raich PC, et al. Conditional survival and the choice of conditioning set for patients with colon cancer: an analysis of NSABP trials C-03 through C-07. J Clin Oncol. 2010;28(15):2544-2548.

14. Guideline Mammacarcinoma 2012 the Netherlands the Netherlands2012. Available from: http://richtlijnendatabase.nl/en/richtlijn/breast_cancer/locoregional_treatment.html.

15. Moossdorff M, van Roozendaal LM, Strobbe LJ, Aebi S, Cameron DA, Dixon JM, et al. Maastricht Delphi consensus on event definitions for classification of recurrence in breast cancer research. J Natl Cancer Inst. 2014;106(12).

16. Lowery AJ, Kell MR, Glynn RW, Kerin MJ, Sweeney KJ. Locoregional recurrence after breast cancer surgery: a systematic review by receptor phenotype. Breast Cancer Res Treat. 2012;133(3):831-841.

17. Metzger-Filho O, Sun Z, Viale G, Price KN, Crivellari D, Snyder RD, et al. Patterns of Recurrence and outcome according to breast cancer subtypes in lymph node-negative disease: results from international breast cancer study group trials VIII and IX. J Clin Oncol. 2013;31(25):3083-3090.

18. van Roozendaal LM, Smit LH, Duijsens GH, de Vries B, Siesling S, Lobbes MB, et al. Risk of regional recurrence in triple-negative breast cancer patients: a Dutch cohort study. Breast Cancer Res Treat. 2016;156(3):465-472.

19. Matsen C, Villegas K, Eaton A, Stempel M, Manning A, Cody HS, et al. Late Axillary Recurrence After Negative Sentinel Lymph Node Biopsy is Uncommon. Ann Surg Oncol. 2016;23(8):2456-2461. 
20. Giuliano AE, Ballman K, Beitsch P, Whitworth PW, Blumencranz P, Leitch AM, Saha S, McCall LM, Morrow M. Ten-year survival results of ACOSOG Z0011: A randomized trial of axillary node dissection in women with clinical T1-2 NO MO breast cancer who have a positive sentinel node. [Abstract]. In press 2016.

21. Pagani O, Price KN, Gelber RD, Castiglione-Gertsch M, Holmberg SB, Lindtner J, et al. Patterns of recurrence of early breast cancer according to estrogen receptor status: a therapeutic target for a quarter of a century. Breast Cancer Res Treat. 2009;117(2):319-324.

22. Saphner T, Tormey DC, Gray R. Annual hazard rates of recurrence for breast cancer after primary therapy. J Clin Oncol. 1996;14(10):2738-2746. 


\section{Appendix 8.1}

Patient demographics and primary tumor characteristics cT1-2N0 per subtype

\begin{tabular}{|c|c|c|c|c|c|c|}
\hline & $\begin{array}{l}\text { All patients } \\
(\mathrm{N}=18,009)\end{array}$ & $\begin{array}{c}\text { ER+PR+Her2- } \\
(N=9,929)\end{array}$ & $\begin{array}{l}\text { ER+PR-Her2- } \\
(\mathrm{N}=2,032)\end{array}$ & $\begin{array}{l}\mathrm{ER}+\mathrm{Her} 2+ \\
(\mathrm{N}=1,231)\end{array}$ & $\begin{array}{c}\text { ER-Her2+ } \\
(\mathrm{N}=667)\end{array}$ & $\begin{array}{l}\text { Triple negative } \\
\qquad(\mathrm{N}=1,701)\end{array}$ \\
\hline $\begin{array}{l}\text { Age, in years } \\
\text { median (range) }\end{array}$ & $59(22-98)$ & $59(22-95)$ & $62(23-91)$ & $54(24-88)$ & $57(30-89)$ & $54(30-89)$ \\
\hline \multicolumn{7}{|l|}{ Tumor type, n (\%) } \\
\hline ductal & $13640(75.7)$ & $7299(73.5)$ & $1454(71.6)$ & $1064(86.5)$ & $606(90.8)$ & $1403(82.5)$ \\
\hline lobular & $1858(10.3)$ & $1205(12.1)$ & $291(14.3)$ & $57(4.6)$ & $3(0.5)$ & $35(2.1)$ \\
\hline mixed or other & $2511(14.0)$ & $1425(14.4)$ & $287(14.1)$ & $110(8.9)$ & $58(8.7)$ & $263(15.4)$ \\
\hline \multicolumn{7}{|l|}{ Grade, n (\%) } \\
\hline 1 & $4730(26.3)$ & 3344 (33.7) & $568(28.0)$ & $130(10.6)$ & $17(2.6)$ & $61(3.6)$ \\
\hline 11 & $7774(43.2)$ & $4732(47.6)$ & 952 (46.9) & $521(42.3)$ & $161(24.1)$ & $293(17.2)$ \\
\hline III & $4872(27.0)$ & $1558(15.7)$ & $434(21.3)$ & $547(44.4)$ & $480(72.0)$ & $1292(76.0)$ \\
\hline unknown & $633(3.5)$ & $295(3.0)$ & $78(3.8)$ & $33(2.7)$ & $9(1.3)$ & $55(3.2)$ \\
\hline \multicolumn{7}{|l|}{ cT-stadium, n (\%) } \\
\hline cT1N0 & $13809(76.7)$ & 7930 (79.9) & $1558(76.7)$ & $890(72.3)$ & $405(60.7)$ & $1123(66.0)$ \\
\hline cT2NO & $4200(23.3)$ & $1999(20.1)$ & $474(23.3)$ & $341(27.7)$ & $262(39.3)$ & $578(34.0)$ \\
\hline \multicolumn{7}{|l|}{ pT-stadium, n (\%) } \\
\hline рTO & $1(0.0)$ & $0(0.0)$ & $1(0.05)$ & $0(0.0)$ & $0(0.0)$ & $0(0.0)$ \\
\hline pT1 & $12332(68.5)$ & 7111 (71.6) & $1381(68.0)$ & $738(63.6)$ & $360(54.0)$ & $955(56.1)$ \\
\hline рT2 & $5422(30.1)$ & $2692(27.1)$ & $624(30.7)$ & $431(35.0)$ & $294(44.1)$ & $723(42.5)$ \\
\hline pT3 & $157(0.9)$ & $81(0.8)$ & $18(0.9)$ & $12(1.0)$ & $9(1.3)$ & $18(1.1)$ \\
\hline pT4 & $18(0.1)$ & $7(0.1)$ & $1(0.05)$ & $1(0.1)$ & $1(0.1)$ & $2(0.1)$ \\
\hline unknown & $79(0.4)$ & $38(0.4)$ & $7(0.3)$ & $4(0.3)$ & $3(0.5)$ & $3(0.2)$ \\
\hline \multicolumn{7}{|c|}{ Surgical treatment, n (\%) } \\
\hline breast conserving & $12173(67.6)$ & $6887(69.4)$ & $1329(65.4)$ & $775(63.0)$ & $367(55.0)$ & $1185(69.7)$ \\
\hline mastectomy & $5836(32.4)$ & $3042(30.6)$ & 703 (34.6) & $456(37.0)$ & $300(45.0)$ & $516(30.3)$ \\
\hline \multicolumn{7}{|l|}{ SLN, n (\%) } \\
\hline negative & $12292(68.3)$ & $6608(66.6)$ & $1397(68.8)$ & $820(66.6)$ & $475(71.2)$ & $1268(74.5)$ \\
\hline micrometastasis & $1322(7.3)$ & $826(8.3)$ & $136(6.7)$ & $87(7.1)$ & $47(7.0)$ & $83(4.9)$ \\
\hline macrometastasis & $3056(17.0)$ & $1821(18.3)$ & $346(17.0)$ & $253(20.5)$ & $111(16.7)$ & $213(12.5)$ \\
\hline unknown & $1339(7.4)$ & $674(6.8)$ & $153(7.5)$ & $71(5.8)$ & $34(5.1)$ & $137(8.1)$ \\
\hline \multicolumn{7}{|c|}{ ALND performed if SLN+, $n(\%)$} \\
\hline yes & 3966 (90.6) & $2376(89.8)$ & $431(89.4)$ & $317(93.2)$ & $146(92.4)$ & $274(92.6)$ \\
\hline no & $412(9.4)$ & $271(10.2)$ & $51(10.6)$ & $23(6.8)$ & $12(7.6)$ & $22(7.4)$ \\
\hline \multicolumn{7}{|l|}{ pN-stadium, n (\%) } \\
\hline pNO & $13177(73.2)$ & 7036 (70.9) & $1491(73.4)$ & $862(70.0)$ & $494(74.1)$ & $1373(80.7)$ \\
\hline $\mathrm{pN} 1 \mathrm{mi}$ & $1211(6.7)$ & $739(7.4)$ & $131(6.4)$ & $80(6.5)$ & $41(6.2)$ & $75(4.4)$ \\
\hline pN1a & $2813(15.6)$ & $1716(17.3)$ & $319(15.7)$ & $208(16.9)$ & $95(14.2)$ & $183(10.8)$ \\
\hline pN1b & $29(0.1)$ & $14(0.1)$ & $3(0.2)$ & $5(0.4)$ & $1(0.1)$ & $4(0.3)$ \\
\hline $\mathrm{pN} 1 \mathrm{c}$ & $47(0.3)$ & $30(0.3)$ & $6(0.3)$ & $1(0.1)$ & $1(0.1)$ & $2(0.1)$ \\
\hline $\mathrm{pN} 2$ & $519(2.9)$ & $292(3.0)$ & $51(2.5)$ & $53(4.3)$ & $23(3.5)$ & $41(2.4)$ \\
\hline pN3 & $177(1.0)$ & $83(0.8)$ & $27(1.3)$ & $21(1.7)$ & $11(1.7)$ & $16(0.9)$ \\
\hline unknown & $36(0.2)$ & $19(0.2)$ & $4(0.2)$ & $1(0.1)$ & $1(0.1)$ & $7(0.4)$ \\
\hline \multicolumn{7}{|l|}{ Chemotherapy, n (\%) } \\
\hline yes & $5767(32.0)$ & $2578(26.0)$ & $463(22.8)$ & $600(48.7)$ & 453 (67.9) & $1095(64.4)$ \\
\hline no & $12242(68.0)$ & $7351(74.0)$ & $1569(77.2)$ & $631(51.3)$ & $214(32.1)$ & $606(35.6)$ \\
\hline \multicolumn{7}{|c|}{$\begin{array}{l}\text { Hormone therapy in case of } E R+, n \\
(\%)\end{array}$} \\
\hline yes & $7102(47.2)$ & $4664(47.0)$ & $951(46.8)$ & $96(64.7)$ & - & - \\
\hline no & 7935 (52.8) & $5265(53.0)$ & $1081(53.2)$ & $435(35.3)$ & - & - \\
\hline \multicolumn{7}{|c|}{$\begin{array}{l}\text { Trastuzumab and chemotherapy, } \\
\text { in case of HER } 2+, n(\%)\end{array}$} \\
\hline yes & $933(87.7)$ & - & - & $526(87.7)$ & $398(87.9)$ & - \\
\hline no & $131(12.3)$ & - & - & $74(12.3)$ & $55(12.1)$ & - \\
\hline
\end{tabular}

Subtype is missing is in $13.6 \%$. N number of cases, ER estrogen receptor, PR progesterone receptor, HER2 human epidermal growth factor receptor 2, cT clinical tumor stadium, pT pathological tumor stadium, SLN sentinel lymph node, ALND axillary lymph node dissection. 


\section{Appendix 8.2}

Multivariable Cox Regression to assess the impact of breast cancer subtype on 5-year regional recurrence as a first event, corrected for confounders

\begin{tabular}{lcccccc}
\hline & \multicolumn{3}{c}{ cT1-2NO patients } & \multicolumn{3}{c}{ pT1-2N+(sn) patients } \\
\cline { 2 - 7 } & HR & $95 \% \mathrm{Cl}$ & $\mathrm{p}$-value & HR & $95 \% \mathrm{Cl}$ & $\mathrm{p}$-value \\
\hline Subtype & & & & & & \\
ER+PR+Her2- & ref & & & & \\
ER+PR-Her2- & 1.943 & $1.225-3.079$ & 0.005 & 2.358 & $0.921-6.035$ & 0.074 \\
ER+Her2+ & 1.926 & $1.028-3.608$ & 0.041 & 0.525 & $0.059-4.644$ & 0.563 \\
ER-Her2+ & 1.415 & $0.604-3.312$ & 0.424 & 1.675 & $0.294-9.539$ & 0.561 \\
Triple negative & 2.477 & $1.442-4.253$ & 0.001 & 2.940 & $1.008-8.574$ & 0.048 \\
Age per year & 0.975 & $0.961-0.989$ & 0.001 & 0.992 & $0.964-1.021$ & 0.591 \\
Grade 1-2 vs 3 & 0.443 & $0.294-0.666$ & 0.000 & 0.174 & $0.080-0.380$ & 0.000 \\
Breast surgery mastectomy vs BCT & 0.605 & $0.302-1.212$ & 0.157 & 0.382 & $0.141-1.034$ & 0.058 \\
pT-stadium T1 vs T2 & 0.506 & $0.355-0.719$ & 0.000 & 0.734 & $0.408-1.320$ & 0.301 \\
pN-stadium N0 vs N1 & 0.400 & $0.278-0.575$ & 0.000 & - & - & - \\
pN-stadium N1 vs N2-3 & - & - & - & 0.536 & $0.231-1.244$ & 0.146 \\
Radiation therapy no vs yes & 2.905 & $1.464-5.763$ & 0.002 & 4.129 & $1.511-11.283$ & 0.006 \\
Chemotherapy no vs yes & 2.701 & $1.656-4.405$ & 0.000 & 2.031 & $0.864-4.777$ & 0.104 \\
Endocrine therapy no vs yes & 2.958 & $1.837-4.763$ & 0.000 & 3.999 & $1.551-10.310$ & 0.004 \\
Trastuzumab no vs yes & 1.369 & $0.579-3.234$ & 0.474 & 1.973 & $0.346-11.236$ & 0.444 \\
\hline
\end{tabular}

ER estrogen receptor, PR progesterone receptor, HER2 human epidermal growth factor receptor 2, BCT breast conserving therapy, pT pathological tumor stadium, pN pathological nodal stadium. 

Summary, discussion and future perspectives 



\section{Summary, discussion and future perspectives}

In summary, this thesis has provided some answers that may help us avoid comparing apples and oranges in breast cancer research.

The first chapter showed that we actually are comparing apples and oranges, even when we focus on local and regional endpoints: many different endpoints are used, definitions are often not provided and if the endpoint is defined, the definition of the same endpoint may vary between studies.

The second chapter showed that worldwide experts in the field of breast cancer indeed disagreed at first about definitions of local and regional endpoints, but finally reached consensus on what we should classify as local event, second primary breast cancer, regional event, and distant event in breast cancer studies. Some issues were subject to debate as the expert panel considered that the available evidence was insufficient, namely whether contralateral lymph node recurrences are distant or regional events, whether we should distinguish between "true recurrences" from "ipsilateral second primary breast cancer" when a recurrence in the ipsilateral breast occurs, and whether irresectable recurrences should be considered distant regardless of location.

The third chapter builds forward on the question regarding contralateral lymph nodes. Whether a contralateral lymph node recurrence (CLNR) should be classified as distant or regional depends on its prognostic impact. This systematic review showed that prognosis of published cases of CLNR was more similar to regional events than distant events, namely $82.6 \%$ overall survival and $65.2 \%$ disease-free survival after a median follow up of 50 months. It also suggested that CLNR alone without any other recurrence has inferior prognosis compared to CLNR and simultaneous ipsilateral breast tumor recurrence (IBTR).

Because this review was limited by a small number of patients and a high risk of publication bias and therefore at risk overestimation of prognosis, Chapter 4 explores prognosis of CLNR in a larger population. It includes data from two national cancer registries and three individual hospitals. In contrast to the systematic review, this population had OS of only $30.2 \%$ after 5 years. This was worse compared to prognosis after ipsilateral lymph node recurrences (5-year OS 57.4\%) but better compared to distant metastasis (5-year OS 10.1\%). The study was limited by the fact that the CLNR population was diagnosed earlier (20\% before 1995), potentially leading to underestimation of prognosis due to suboptimal treatment of both the initial cancer and 
the CLNR. Furthermore, information on both the presence of IBTR, and influence of detection method (repeat SN versus clinically evident CLNR) was lacking, and no conclusions could be drawn about those situations. Despite that, this chapter suggests that prognosis after CLNR is not as good as after ILNR, but considerably better than after distant metastasis. Therefore, all CLNRs are not necessarily similar to ILNR, but curative treatment may be suitable for individual patients.

Chapter 5 builds further on classification of lymph nodes, but focuses on infraclavicular lymph nodes at initial diagnosis. If affected they are classified as pN3a according to TNM, similar to presence of $>10$ affected axillary lymph nodes. This chapter shows that prognosis of patients staged as pN3a based on infraclavicular nodes is better than prognosis of patients staged pN3a based on $>10$ axillary lymph nodes, and suggests that the next TNM classification should not classify them in the same category.

In conclusion, the first part of this thesis shows that in breast cancer research, many different endpoints are used and there is a need for more consistent definition. Regarding some issues with classification, evidence was unavailable, and Chapters 3-5 provide some answers to these questions.

The second part of this thesis focused on individual risk and the timing of local and regional recurrence. A first step towards more individual risk assessment is using the characteristics of tumor biology that we routinely measure in breast cancer patients: hormone and Her2 receptor status. We can divide tumors into subtypes with different biologic behavior and different response to therapy.

Chapter 6 studied the risk of local recurrence after mastectomy in these different subtypes. This study showed that these subtypes are important: their absolute risk of LR varies (triple negative tumors were at the highest risk), and also different risk factors are important in different subtypes. Furthermore, other studies have suggested that different subtypes may respond differently to treatment such as radiation therapy. This means that the decision which patients need local treatment such as radiation therapy should be tailored to subtype, and research investigating local treatment should describe the results separately for different subtypes of breast cancer so we can do better in the future.

Chapter 7 and 8 focus on the timing of local and regional recurrences in different subtypes of breast cancer. We often express prognosis in terms of 5-year risks, for instance "the 5-year risk of local recurrence is 3\%". But if a woman has finished treatment and has been breast cancer free for three years, is her risk of LR in the next 2 
years still 3\%? As an answer to this question, Chapter 7 firstly revealed that the risk of local recurrence in the first 5 years after diagnosis was already quite low at $3 \%$ directly after treatment. Secondly, after 3 event-free years, the risk of LR in the next two years was only 1\%. A similar pattern was seen in Chapter 8 for regional recurrence (RR). The different subtypes showed different patterns of recurrence for both LR and RR: the subtypes with the highest risks (triple negative and Her2+ breast cancer) showed the fastest decline. This information can be reassuring to individual patients who have remained event free for a number of years. It also suggests that follow-up beyond 3 years may have low yield (although recurrences do happen). This is particularly important for breast cancer research, to estimate whether continued follow up will change the message of the study. This may lead to acceptance of earlier results, although longer follow-up may be necessary for other outcomes and for ER+ breast cancer. In individual patients, this information may be used as a starting point to tailor follow-up to individual needs, although there are many reasons for prolonged follow-up besides detecting local and regional recurrences.

In future breast cancer research, these findings may have a positive influence on the quality and fast availability of reliable results. Using clear and consistent definitions throughout breast cancer research will facilitate reliable comparison of results. Tailoring follow-up to subtypes of breast cancer is a first step towards reacting to the biologic behavior of the tumor, instead of a one-size-fits-all approach. The low absolute yield (as a result of the low number of events) may lead to evaluating (preliminary) outcomes after 3 years instead of 5 or 10 (at least for local endpoints). This may speed up certain studies although it will certainly not be possible for every trial.

Particularly the low risk of recurrence will be a challenge for future breast cancer research, as this will make it difficult to obtain statistically significant results. The focus on statistical significance of the results and the lack of power due to low number of events, however, sometimes distracts our attention from the actual size of the benefit. Lack of power because not the expected $5 \%$ but only $1 \%$ developed a recurrence, does not mean that the study is of low quality; it means that both treatments were really good. Furthermore, a difference between two interventions (even if statistically significant) may be so small, that we do not consider it clinically relevant. A very striking example was the ACOSOG Z11 study, ${ }^{1,2}$ randomizing women with cT1-2NO breast cancer and 1-2 positive sentinel nodes after breast conserving therapy to either watchful waiting or axillary lymph node dissection). The study was closed early because of slow accrual and consequently, it was underpowered and no significant difference was seen between the treatment arms. If we look more closely at the actual risks, the absolute 5 -year risk of regional recurrence was $0.9 \%$ without ALND and $0.5 \%$ with ALND after a 
median follow-up of 6.3 years. This difference was not significant, but had it been, it would not be clinically relevant and certainly not justify exposing all patients to the potential morbidity of ALND. Additionally, other meaningful outcomes such as OS and DFS were also not significant. The authors concluded that although prognosis is inferior in women having 1-2 positive sentinel nodes (compared to women without affected sentinel nodes), the axillary lymph node dissection did not improve this prognosis. Despite this, the results have not been implemented in our standard of care in The Netherlands.

When facing the challenge of low event rates, underpowered studies and non-significant results, we should be more flexible than to dismiss a study simply because few events prevented statistical significance. We should focus more on actual results and find new ways to reliably compare treatments.

This study brings us to a second challenge (or opportunity) for future breast cancer research. As Monica Morrow commented on the results of this ACOSOG Z11 so clearly: "Bigger surgery doesn't overcome bad biology". But how do we overcome bad biology? Can we recognize it? Can we target it to treat the cancer? First steps can be taken if we take breast cancer subtype into account, for instance based on receptor status (such as in Part 2 of this thesis). This information is already available for all breast cancer patients diagnosed today. Larger steps have already been taken by genetic profiling of individual tumors, and these tests are even commercially available at this time. Even bigger steps are being taken by studying the tumor even more closely, and find out what is actually happening on a molecular level within the tumor before, during, and after treatment. Through dedicated research, we are slowly learning what these tests mean for prognosis and for predicting which patients benefit from which treatment.

I believe this is the future of breast cancer treatment, but these studies will face the challenge of long follow-up and low event rates. Carefully choosing endpoints, ensuring clear endpoint definitions, balancing the expected yield of continued follow-up and reliable results, and dealing with low event rates in a way that benefits patients most are the keys towards better, faster, and stronger results for breast cancer patients. 


\section{References}

1 Giuliano AE, Hunt KK, Ballman KV, Beitsch PD, Whitworth PW, Blumencranz PW, et al. Axillary dissection vs no axillary dissection in women with invasive breast cancer and sentinel node metastasis: a randomized clinical trial. JAMA. 2011;305:569-575.

2 Giuliano AE, McCall L, Beitsch P, Whitworth PW, Blumencranz P, Leitch AM, et al. Locoregional recurrence after sentinel lymph node dissection with or without axillary dissection in patients with sentinel lymph node metastases: the American College of Surgeons Oncology Group 20011 randomized trial. Ann Surg. 2010 252:426-432-discussion432-433. 



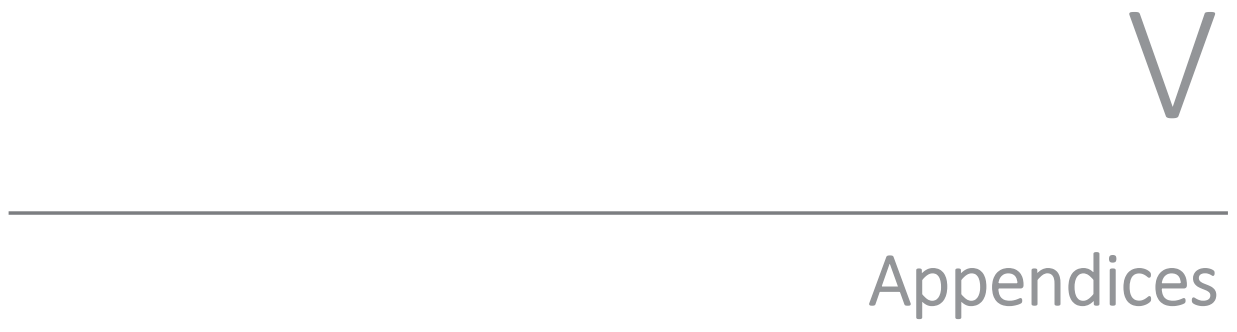



Nederlandse samenvatting 


\section{Nederlandse samenvatting}

Samengevat kan dit proefschrift ons ten eerste helpen om in borstkankeronderzoek niet langer appels met peren te vergelijken en ten tweede om de lange tijd waarin we mensen volgen om te kijken of de ziekte terugkomt, beter op de individuele patiënt af te stemmen.

In Hoofdstuk 1 van dit proefschrift blijkt uit een literatuurstudie dat borstkankeronderzoekers veel verschillende uitkomstmaten gebruiken, dat definities van de uitkomstmaat niet altijd gegeven worden en dat als deze gegeven worden, zij ook nog kunnen verschillen per studie. Dit kan tot gevolg hebben dat studies onderling niet goed te vergelijken zijn. Als we de resultaten toch naast elkaar zetten, trekken we daardoor mogelijk niet de goede conclusies.

Hoofdstuk 2 heeft tot doel een oplossing te bieden voor dit probleem. Een panel van internationale experts nam deel aan een consensusproject, om afspraken te maken over de definities van lokale en regionale terugkeer (ook wel recidief) van borstkanker. Zij waren het eerst niet over alle definities eens, maar uiteindelijk bereikten zij consensus over wat precies een lokaal recidief, een regionaal recidief, een tweede primaire borstkanker en een uitzaaiing op afstand is. Op basis hiervan wordt in Hoofdstuk 2 een voorstel gedaan voor gestandaardiseerde definities voor deze uitkomstmaten.

Hoofdstuk 3 bouwt verder voort op een van de twijfelgevallen die de experts hadden geïdentificeerd in Hoofdstuk 2. Het komt voor dat borstkanker terugkomt in een lymfeklier aan de andere zijde dan de borstkanker (dus een lymfeklier in de linker oksel, terwijl de borstkanker rechts zat). Het was onduidelijk of we deze klieren moesten beschouwen als uitzaaiing of als een 'regionaal' probleem, vergelijkbaar met recidief in een lymfeklier aan dezelfde zijde als de borstkanker. Een manier om die knoop door te hakken, is de prognose van lymfeklierrecidief aan de andere zijde te vergelijken met een lymfeklierrecidief aan dezelfde zijde, en een uitzaaiing op afstand. Hoofdstuk 3 zet alle wetenschappelijke artikelen die hierover verschenen zijn op een rij, waaruit blijkt dat de totale overleving van patiënten met lymfeklierrecidief aan de andere zijde na 6 jaar 82.6\% was en de ziektevrije overleving 65.2\%. Dit komt meer overeen met een lymfeklierrecidief aan dezelfde zijde dan met uitzaaiingen op afstand. Beperkingen van dit onderzoek waren een klein aantal patiënten en het feit dat veel van deze publicaties niet bedoeld waren om deze prognose te bepalen en dus mogelijk niet alle patiënten beschreven worden. Hierdoor kan er overschatting van de prognose plaatsvinden.

Om die reden hebben we in Hoofdstuk 4 de prognose opnieuw bepaald uit kankerregistraties (Nederland, Denemarken) en enkele individuele ziekenhuizen. Hieruit bleek dat de prognose van lymfeklierrecidief aan de andere zijde slechter was dan in 
Hoofdstuk 3 gevonden werd, namelijk 30.2\% totale overleving na 5 jaar, en ergens tussen de prognose van een lymfeklierrecidief aan dezelfde kant (57.4\% 5-jaars overleving) en een uitzaaiing (10.1\% 5-jaars overleving) in zat. Ook deze studie had beperkingen, waaronder ontbrekende gegevens die hadden kunnen helpen verklaren waarom de prognose beter of slechter was en het feit dat de gebruikte gegevens wat ouder waren. Deze beperkingen kunnen er toe leiden dat de prognose in deze studie juist onderschat wordt. Hoofdstuk 3 en 4 wijzen er dus op dat de prognose van een lymfeklierrecidief aan de andere zijde ongunstiger is dan een lymfeklierrecidief aan dezelfde zijde, maar gunstiger dan een uitzaaiing op afstand. Al zijn de twee soorten recidief dus niet hetzelfde, behandelen met genezen als doel ligt bij veel patiënten wel voor de hand.

Hoofdstuk 5 focust ook op classificatie van lymfeklieren, maar dan bij initiële diagnose. Het gaat hier om lymfeklieren die onder het sleutelbeen gelegen zijn. In het officiële classificatiesysteem (TNM) staan die qua ernst gelijk aan aanwezigheid van meer dan 10 aangedane klieren in de oksel. Het onderzoek in Hoofdstuk 5 laat zien dat het weliswaar zeldzaam is dat alleen op basis van de klier onder het sleutelbeen de hoge classificatie wordt gekozen, maar dat de prognose van die patiënten wel beter is dan de patiënten met meer dan 10 klieren in de oksel. Die twee groepen behoren dus niet in een categorie geclassificeerd te worden.

Kortom, de eerste vijf hoofdstukken laten zien dat we helaas vaak appels met peren vergelijken in borstkankeronderzoek en stelt gestandaardiseerde definities voor om dat de voorkomen in de toekomst. Daarnaast worden antwoorden gegeven op enkele van de discussiepunten over classificatie, waar wetenschappelijke gegevens nog voor ontbraken.

Het tweede deel van dit proefschrift focust op individueel risico voor terugkeer en de timing daarvan. Een eerste stap om dat risico beter in te schatten is te kijken naar de receptoren op borstkankercellen. De combinatie van receptoren zegt iets over het biologisch gedrag van de tumor, bijvoorbeeld agressief of juist relatief gunstig. Deze receptoren (oestrogeen, progesteron en HER2) bepalen we al jaren routinematig voor alle nieuwe borstkankers. Op basis van de combinatie van receptoren delen we de tumoren in subtypes in.

Hoofdstuk 6 kijkt naar de kans op lokaal recidief na het verwijderen van de hele borst bij de verschillende subtypes borstkanker. Het blijkt dat die kans inderdaad verschilt per subtype. Gemiddeld was de kans op lokaal recidief 3.8\% in 5 jaar. De kans was het laagst bij de hormoongevoelige (ER+PR+) maar Her2 negatieve tumoren (namelijk 2.8\%) en het 
hoogst bij patiënten met een tumor die negatief is voor alle drie de receptoren (namelijk 9.5\%). Deze resultaten zijn ook een ingang voor verder onderzoek naar de gevoeligheid van de verschillende subtypes voor andere behandelingen zoals bestraling.

Hoofdstukken 7 en 8 kijken naar de timing van de recidieven, uitgesplitst naar subtype, respectievelijk voor lokale en regionale recidieven. We kijken in onderzoek vaak naar "de recidiefkans of sterftekans binnen 5 jaar", maar als een vrouw 3 jaar na de behandeling nog geen recidief heeft, is dat dan een gunstig teken of is de kans nog hetzelfde als bij diagnose? Het blijkt dat zowel voor lokale als regionale recidieven het 5 -jaars risico bij diagnose laag was (3\% lokaal recidief en 1.3\% regionaal recidief in 5 jaar). De kans verschilt ook hier per subtype waarbij de kansverdeling vergelijkbaar is met hoofdstuk 6 : de minste recidieven traden op bij de hormoongevoelige en Her2 negatieve tumoren (2.2\% lokaal en $0.8 \%$ regionaal) en de meeste bij de tumoren die negatief waren voor alle drie de receptoren (6.8\% lokaal en 3.7\% regionaal). Daarnaast bleek dat het risico op lokaal en regionaal recidief afneemt met de ziektevrije jaren en dat die afname het snelst is in subtypes met het hoogste risico bij diagnose. Na 3 ziektevrije jaren was de kans om in de volgende 2 jaar nog een lokaal recidief te krijgen 1\% of minder in alle subtypes (behalve het ongunstigste type met $1.6 \%$ ), en de kans om een regionaal recidief te krijgen minder dan $1 \%$ in alle subtypes.

Deze gegevens zijn belangrijk voor individuele patiënten, omdat dit geruststelling kan bieden en een meer gepersonaliseerd beeld van hun prognose oplevert. Deze informatie kan, in combinatie met andere gegevens en voorkeuren, eventueel ook meegenomen worden in de beslissing om controle in het ziekenhuis te verkorten. Ook in borstkankeronderzoek is de timing van het optreden van recidieven belangrijk, omdat de follow-up duur bepaalt hoe snel de resultaten beschikbaar zijn en omdat een langere duur vaak hoge kosten met zich meebrengt. Voor borstkankeronderzoeken die specifiek naar lokale en regionale recidieven kijken, kunnen deze gegevens de onderzoekers helpen bepalen hoeveel follow-up tijd nodig is voor betrouwbare resultaten. Als dat korter zou kunnen, kan dat niet alleen kostenbesparing beteken maar ook mogelijk eerder beschikbaarheid van data voor de behandeling van patiënten. Voor andere uitkomstmaten, evenals monitoring van bijvoorbeeld hormonale therapie kan het uiteraard wel nodig zijn om patiënten langer op te volgen.

Samengevat is dit proefschrift is een stap naar verbetering van borstkankeronderzoek. Uniforme definities verhogen de kwaliteit en betrouwbaarheid van onderzoeksresultaten, en daarmee ook die van het advies dat we aan patiënten geven. Hetzelfde geldt voor de aandacht voor het biologisch gedrag van de tumor. Dat is in dit proefschrift nog gebaseerd op receptoren, maar in de toekomst zal waarschijnlijk een nog gedetailleerder onderscheid mogelijk zijn. 

Valorisation 



\section{Valorisation}

Valorisation of knowledge means how we create meaningful information from the facts, by presenting it and making it applicable for societal and economic utilization, and by translating it to new business, products, services or processes. ${ }^{1}$

This valorisation chapter will explore how the world outside academia can benefit from this thesis and which new developments might evolve from the generated knowledge.

\section{Economic relevance and relevance to society}

This thesis concerns breast cancer and breast cancer research. Breast cancer is the most common type of cancer in women and the incidence in the Netherlands is approximately 14.500 per annum in The Netherlands. ${ }^{2}$ It is hard to estimate the total amount of funding invested in breast cancer research, but there are (fortunately) countless governmental and non-governmental foundations, charities, and societies supporting breast cancer research worldwide. Searching 'breast cancer' yields, as of August 2017, 346.174 hits on PubMed. This illustrates that achieving better, faster, and stronger results in breast cancer research is not only personally relevant for many, many women and their families confronted with breast cancer, but also for the thousands of citizens and governments investing in breast cancer research.

This issue has become more stressing over the past decade and will become more stressing in the future. The success of breast cancer research in the past few decades has led to few recurrences and very good survival for most breast cancer patients. This means that in the current era, large sample sizes and long follow up are necessary for reliable results. Critically reviewing how we can optimize research by using uniform endpoints and classifications, reconsidering follow up time and finding creative ways to produce reliable evidence with smaller sample sizes will be a necessity for sustainable future breast cancer research. This thesis provides steps towards that goal.

\section{Implications for new initiatives and innovation}

This research can be applied in several ways. First, this thesis generated more detailed prognostic information (i.e. by breast cancer subtype). This information can be integrated in prognostic models that are used to advise individual patients about their treatment. An example is Adjuvant! online. ${ }^{3}$ Integrating the new prognostic information provides patients with more tailored and therefore more accurate information.

Furthermore, this thesis contains new information on prognosis of metastases in contralateral lymph nodes and infraclavicular lymph nodes. This information may be 
used to improve the next version of the TNM classification ${ }^{4}$ of breast cancer, which is used by physicians and researchers throughout the world.

The knowledge generated in this thesis can also help make research easier, more efficient, and more transparent. The best way to achieve this, would be a uniform format for data collection on a nationwide or even worldwide level. A first step could be a mobile application or website which could be used by researchers, data managers and physicians to classify a breast cancer recurrence (for instance according to the standardized definitions from Chapter 2).

The next step (for which more knowledge, software, logistics, and commitment from stakeholders worldwide would be required) should, in my opinion, go towards a nationwide or worldwide, standardized database. This should safely store data with regard to privacy and sensitive information, be affordable and collect all the information that we need to move forward. Current cancer registries and clinical trial data management strategies hold an enormous wealth of information, but still have disadvantages, particularly the fact that they are not standardized (i.e. data are collected in a slightly different way) and can be inefficient, which all makes them are very costly. There are currently several web based and tablet based applications that safely store research data using standardized forms, and some of which can be linked to patient records. This is a huge step forward. However, these are more difficult to integrate and do not necessarily communicate. In the era of transparency and open access, I think uniform data collection (based on international consensus) and safe storage are the next step.

\section{Realisation}

Implementation of new data in guidelines and classification systems works through publication in peer reviewed journals and presentation of results on international platforms. If the information is available and awareness is created, the data will be weighed to the total body of evidence and implemented as appropriate.

Implementation of uniform endpoint definitions particularly needs awareness among clinicians, researchers, but also providers of grants, trial registries and journals, which can demand certain definitions or at least specifications. Furthermore, use of definitions in a final paper also requires that specific data were collected. This means that endpoint definitions should optimally be chosen before initiating the study. This also means that if definitions are implemented today in all new research protocols, it may take several years before we can compare studies that used these standardized definitions.

Implementation of standardized data registration internationally or nationally is an extremely large and extremely costly project. Creating an application that would allow safe and standardized collection of patient data, preferably being able to extract 
information directly from electronic patient's records as soon as they enter a study and/or give permission, and if possible specific for breast cancer research purposes and at a reasonable price would result in a dramatic improvement in efficiency.

In summary, improving efficiency of breast cancer research means anticipating on future challenges of trials requiring large sample sizes at high costs. Such efficiency will be beneficial to society: both for breast cancer patients and their families, as well as on an economic level. Furthermore, the generated knowledge can be implemented in guidelines and classification systems. In the future, applications that further standardize data collection based on international consensus, that allow more efficient pooling and exchange of results, would be a huge leap forward. 


\section{References}

1. Report 'Van voornemens naar voorsprong: kennis moet circuleren - voorstel voor een Nederlandse valorisatieagenda'. Waas, 2010. https://www.rvo.nl/sites/default/files/bijlagen/Rapport\%20kennis\%20 moet\%20circuleren.pdf

2. Cijfers over kanker. Incidentie borstkanker http://www.cijfersoverkanker.nl/selecties/ incidentie_ borst/img568b9b027142c

3. Adjuvant! Online. https://www.adjuvantonline.com

4. Edge SB, Compton CC. The American Joint Committee on Cancer: the 7th edition of the AJCC cancer staging manual and the future of TNM. Annals of surgical oncology. 2010; 17:1471-1474. 
Dankwoord 



\section{Dankwoord}

It was the best of times, it was the worst of times... Maar het is voltooid! Er zijn een aantal mensen zonder wie ik niet gekomen was waar ik nu ben, zonder wie dit proefschrift er nooit geweest was, zonder wie het promoveren beduidend minder leuk was geweest, mensen die er op het goede moment altijd blijken te zijn, en een paar mensen die in al die categorieën vallen. Dit boekje is niet compleet zonder jullie.

Dr. Smidt, Beste Marjolein, je hebt geen idee hoeveel ik van je geleerd heb. Ik kwam als zesdejaars student solliciteren omdat ik het idee van de BOOG-studie (destijds nog SeNoMore) zo'n "goed onderzoeksdoel" vond. Zo blij dat ik de gelegenheid had om aan te sluiten bij het groepje dat je om je heen had verzameld. Het doorlopend thema van je wijze lessen is voor mij ongetwijfeld "Je moet stoppen als het af is" wat natuurlijk ook buiten onderzoek erg breed toepasbaar is (mijn badkamerkraan is je eeuwig dankbaar). Een andere wijze les is de kunst van het presenteren en grants schrijven, ofwel mensen meenemen in je enthousiasme, transparant en eerlijk, maar wel zorgen dat bescheidenheid of correctheid je niet onterecht tegenwerken ("Jij kunt gewoon geen auto zonder motor verkopen", "Ik wil ook helemaal geen auto zonder motor verkopen"). Daarnaast hoe om te gaan met het feit dat vrouwen onderling soms nogal bitchy zijn als ze concurrentie ervaren, en dat caviaraces en sinaasappels volledig algemeen geaccepteerd zijn in wetenschappelijke presentaties. Heb ik, ondanks mijn milde eigenwijsheid, de boodschap toch een beetje begrepen? Dankjewel voor je grenzeloze en stimulerende enthousiasme, de wijze lessen dus, alle fantastische kansen die je me gegund hebt de afgelopen jaren en natuurlijk de onderzoekers-etentjes (maar dan krijgt Ivo ook credits voor de oesters en Linde, Nienke en Gijs voor hun trampoline-skills).

Dr. Strobbe, beste Luc, vanaf het begin was je met je kritische blik een extreem waardevol onderdeel van mijn promotieteam. Jaren ervaring als chirurg en als onderzoeker zorgden meerdere malen voor het tegenwicht dat de stukken nodig hadden om beter te worden. Prof. Stassen, hartelijk dank voor de steun en het vertrouwen, natuurlijk met name in de laatste fase. Dank voor uw constructieve bijdrage, ik kijk uit naar de volgende samenwerking in de kliniek!

Natuurlijk ook de Leden van de beoordelingscommissie: Prof. Beets, beste Geerard, tijdens mijn semi-arts periode in het MUMC heb ik veel van $u$ geleerd over wat een goede dokter is en in mijn huidige baan blijkt dat de patiënten in de regio u nog steeds missen. Na onze korte samenwerking aan het begin van het traject was ik meer dan vereerd dat u wilde deelnemen aan de beoordelingscommissie. Dr. Roumen, beste Rudi, 
hartelijk dank voor het beoordelen van mijn proefschrift, ik heb altijd veel waardering voor uw scherpe vragen en opmerkingen aan de microfoon bij congressen en refereeravonden, wat u natuurlijk een ideale beoordelaar en opponent maakt. Prof. Heeren, Beste Ron, sinds je komst op het lab heb je me meerdere malen verrast met je rust en enthousiasme. De mix van expertise, onderzoek op hoog niveau, waardering voor je team, je enthousiasme waaruit men alleen maar kan concluderen dat je de leukste baan van de wereld hebt, ik vind je een bewonderenswaardig onderzoeker en persoon. Prof. Ramaekers, beste Frans, u bedank ik dubbel, niet alleen voor het vervullen van het voorzitterschap van de beoordelingscommissie maar ook omdat $u$ als directeur van GROW mij en vele medepromovendi zo veel kansen aanreikt om ons verder te ontwikkelen. Dan ontbreken natuurlijk ook Brigitte en Judith niet, jullie hebben ons meerdere malen op het laatste moment geholpen, ook met de grootste successen.

Zonder het vertrouwen van Kankeronderzoeksfonds Limburg was dit proefschrift er ook niet geweest. Speciaal voor Babette Frank, Marcel Bourgonje en Ellen van de Ven: hartelijk dank, voor jullie inzet voor wat deze regio te bieden heeft op onderzoeksgebied, maar ook voor de leuke activiteiten.

Het Borstkanker-team in het MUMC: Marc Lobbes, Esther Heuts, Kristien Keymeulen, Prof. Tjan-Heijnen, Maaike de Boer, Prof. Liesbeth Boersma, uiteraard Elly, Jeanine, Conny, Mieke en Christel, en van wisselende duur ook Bart de Vries, Koen van de Vijver, Leonie Smit, Evert-Jan Boerma en Sanne Engelen. Ik heb veel van jullie geleerd! En Sabeth, je zit natuurlijk op de stafgang en niet op het oncologiecentrum, maar jij hoort hier ook zeker bij: dankjewel voor alle (last minute) hulp!

Veel van de gegevens die we voor de inhoud van dit proefschrift hebben kunnen gebruiken zijn afkomstig van IKNL. Sabine Siesling, Marissa van Maaren, Linda de Munck, Reini Bretveld, bedankt voor de prettige samenwerking, ik heb geleerd wat een eindeloze schat aan informatie het jarenlange monnikenwerk oplevert, ik hoop dat iedereen ziet hoe waardevol een goede landelijke registratie is.

Daarnaast Sander van Kuijk, bedankt voor de je statistische relativeringsvermogen en je creatieve oplossingen. Het is echt bewonderenswaardig hoe goed je de wereld van getallen kan vermengen met de wereld van het ziekenhuis.

Beste Guusje, super efficiënte samenwerking voor de review en ook supergezellig, we gaan elkaar nog zien de komende jaren!

Professor King, dear Tari and Dr. Nahklis, dear Faina. Thank you so much for giving me the opportunity of a lifetime by working with your team in one of the most prestigious 
oncology clinics of the world. The dedication of the team at Dana Farber/Brigham and Women's was inspiring, and the experience will definitely shape my future career in Surgery and research.

Zonder Lori en Robert-Jan had ik vast nooit geleerd om een beetje goede stukken te schrijven (of efficiënt om te gaan met bepaalde copromotoren). Lori, tijdens het maken van jouw promotiefilmpje stuitte ik op een eindeloze hoeveelheid beeldmateriaal waaruit bleek wat een toptijd het was: congressen, labuitjes, San Antonio Spurs, cocktails met gummibeertjes, Stromae concerten en nog veel meer. Robert-Jan, ik was altijd heel blij dat je in ruil voor Engelse spelling-checks goede koffie te bieden had (mede mogelijk gemaakt door Rutger), maar ik wil je vooral bedanken dat ik bij je mocht aansluiten voor de MRI-axilla studie, de artikelen en de grant-aanvragen. Het was een vliegende start en die heb ik voor een groot deel aan jou te danken.

Lieve Briete, een beetje een impuls-actie, maar de tussenstop in New York na het congres in Houston was echt het beste idee! In 5 dagen de uiterst culinaire to-eat lijst afgewerkt, onderkoeld geraakt op het Empire State Building, en uiteindelijk twee keer de Shoegasm bereikt. Je bent een ontzettend fijne collega en persoon en wat mij betreft ook een voorbeeld voor het maken van keuzes waar je gelukkig van wordt (ook al is er soms enige twijfel vooraf). Marissa, het was supergezellig jouw collega te zijn, in Boston woonruimte te zoeken (dakterras beats zweverige holistische thee), ijshockey te kijken, op de kamer met het mooiste uitzicht in UNS40 kopjes thee te drinken, natuurlijk ook met Yvonne (nog steeds sorry voor het unpluggen van de koelkast), en ik vergeet nooit hoe je in slaap viel boven SPSS \& de tabellen van hoofdstuk 7 en 8 tijdens de nachtvlucht naar IJsland. Thiemo, jij hebt kwaliteiten die ik echt bewonder omdat ik ze zelf ook meer zou willen hebben, zoals je precisie bij langdurige projecten en routineklussen, je onverstoorbaarheid bij tegenslagen, de rust die je uitstraalt en je tevredenheid over simpele maar belangrijke zaken in het leven. Ik probeer het soms een beetje na te doen goed?

Uiteraard ook de rest aan UNS 40 side: Givan (dikke bas uit de speaker, raampje open, zonnebril op, je werkkamer op vrijdagmiddag hoeft geen onprettige omgeving te zijn), Britt, Miriam en Rianne. Selwyn en Victor, relaxed dat jullie als mede semi's die tegelijk van de semi-tuin naar UNS40 verhuisden... Toch miste ik Tine, Nikki en Lotte wel een beetje in die semi-context (nouja, Diner Les Cons-context in samenwerking met Frans), gelukkig zijn jullie nog steeds in voor diners! Op diezelfde gang was ook Joost te vinden, maar dat wist ik toen niet, gelukkig kom ik die tegenwoordig op een andere gang wel tegen. Dankjewel voor de bananenpannenkoekjes en het lenen van je monitor! 
En natuurlijk gaat UNS40 naadloos over in UNS50, waardoor een koffietje bij Anne-Claire snel geregeld was. AC, je had even tijd nodig had om er achter te komen dat ik niet "te blij" ben, maar nu dat is goedgekomen kan ik rustig zeggen dat je een held bent in al je activiteiten (en heel soms een sukkel), en dat ik superblij ben dat we nog een hele tijd kunnen carpoolen, thee drinken, racefietsen naar werk ( $\mathrm{hmmm}$ ) en jou kennende, so much more. Twee kamers verder natuurlijk Frans (straks sta je in alle stukjes!), toch altijd een mysterie die activiteiten van jou in UNS50, er waren vaak lijstjes, schema's en geodriehoeken bij betrokken, of botten, en als het echt geheimzinnig werd vetrok je naar Venlo om daar om 4.30 AM allerlei zaken te scannen... Ik hoop wel dat je aan het eind van de rit kunt zeggen "No Kerbals were harmed during the production of this thesis". En op dezelfde kamer David, coach van mijn allereerste racefietsrondje waardoor ik dus eigenlijk nooit commentaar op de route zou mogen leveren, tevens onverslagen meester in Geosense en GeoGuessr en oja: Suit up Friday was een daverend succes, maar Topless Tuesday is geen dingetje. En Kim, zo veel gezellige avonden in Thembi en met fietsen, met als doorlopend thema "never go full retard" (met wisselend succes), Irene Fleur, ik ben echt fan van jouw rust en doelgerichtheid en natuurlijk je interieursmaak, en mijn planten krijgen tegenwoordig ook groene thee :):

Hoewel dinsdag gereserveerd was voor Pubquizzen bij Edd's, is woensdag soms het nieuwe vrijdag en dan zou Thembi niet hetzelfde zijn geweest zonder Audrey, Luuk, Kiran, Leontine, Claire, Junfang, Luuk, Jasper, Tim, Joyce-Manyi (iets met Uranus, maar daar hebben we het op de CASH cursus wel over) en later Jacqueline, maar natuurlijk ook Mo, Kaatje, en Bas. Ondanks je houding tegenover handdoeken en fietspakjes heb ik nog steeds geen antwoord op de eeuwige vraag of je erger bent dan Dennis (ik zie nog steeds alleen Mr Big in mijn telefoon staan).

En gelukkig kan ik Charlotte, Milou en AC tegenwoordig opnieuw collega's noemen, fijn dat ik jullie goede voorbeeld kan volgen! Maar natuurlijk ook aan alle andere lieve Collega's van het Zuyderland (bazen, assistenten, verpleegkundigen, polidames, you know who you are), ik ben blij dat ik met jullie de volgende stap mag zetten!

Dan natuurlijk de Checkjes.... wat moet ik zonder jullie! Marieke, van nachtelijke tosti's, huisjes, boompjes en beestjes, dan via een Viking-helm in Turkije tot kano's en wadlopen, ik ben blij dat de wereld niet meer altijd zwart/wit is, en hoewel grijswaarden niet zo sexy klinkt wordt het leven er veel beter van! Sieltje, je verdient misschien de hospital ink-award, kitty lover-award, slechtste-KKB-in-de-persoonlijke-sfeer award, en soms de awkwardness-award, maar ook de always there-award, de carrot cake-award, de speelpakjes-award en de work hard, play hard-award ("ik heb geen hobby's, ik doe onderzoek" was wel aan een tweede druk toe, bij dezen). You're the best. It's true, she's 
the best. Yasmijn, jouw relativeringsvermogen en altijd beschikbare moral support waren echt onmisbaar de afgelopen 4 jaar! Daar voor natuurlijk ook, 'Dierecteuren' en IJscohoofdpijn gaan je ook niet in de koude kleren zitten... maar promotie-specifieke internetvondsten als 'describe your thesis in one sentence' (om zomaar een voorbeeld te noemen: looked for a gene, didn't find it) kunnen net het verschil maken op een dag die anders in het teken zou staan van rejection letters en onopgeslagen worddocumenten. Na de verdediging maar eens een roadtrip in Ferrari 2.0 (alleen gaat ie wat langzamer van 1 naar 100).

Sanne (roomie!!!), hoewel je het soms goed weet te verbergen ben jij echt een ontzettend betrouwbare vriendin en natuurlijk huisgenoot! Er waren misschien kleine meningsverschillen, zoals het vonnis in de zaak Martine vs. Alexander, de juiste plaats om je van GFT-afval te ontdoen, en de timing van het sluiten van je slaapkamerdeur, maar uiteindelijk waren we het altijd eens over special breakfasts, Batman-posters, festivals, misdaadseries op Netflix en het antwoord op de vraag What does the fox say? Best. Roomie. Ever. Beter dat je weer terug bent in Nederland. Sizzle, ook al ben je nu een beetje ver weg en doen we dingen altijd heel anders, je bent ook een voorbeeld en een spiegel!

Inger en Julia, piglets, ik weet nu al dat jullie uitvoering van Het Mannenlied de performance of a lifetime gaat worden (no pressure). Als er twee mensen zijn die mij goed kennen en er altijd zijn, dan zijn jullie het. Dan maakt het dus ook niet uit of het in Clairvaux of in een bedstee is. The Don, Mosse, Patricia en Edelros sluiten zich hier natuurlijk volledig bij aan en verwachten nog vele Biggen-weekendjes in de toekomst.

Sommige mensen mogen twee keer, in dit geval Frans en Yas. Jullie kunnen echt alles. Dankjewel dat ik mezelf en promotieperikelen mocht toevoegen aan jullie eigen lange to do lijstjes, congressen, vakanties, las- en vliegwerk. Dankjewel dat jullie altijd de telefoon opnemen als ik weer eens denk dat iets in de beschikbare tijd gewoon onmogelijk is maar het toch wel moet. Dankjewel dat jullie altijd met iets kleins de dag weten te redden. En dankjewel dat jullie deze (ongetwijfeld fantastische) dag met me willen delen!

Carien, lieve zus, ik ben echt heel blij dat ik je de afgelopen jaren wat meer gesproken en gezien heb! De Marty McFly Welcome Party kwam niet echt van de grond (die was ook wel teleurgesteld geweest in de kwaliteit van de huidige hoverboards) maar elke keer dat ik een Bassie \& Adriaan-waardige bestemming bezoek denk ik aan jou. lemand vroeg "lijkt je zusje eigenlijk op je" en toen zei ik "ze is heel erg anders, maar toch ook wel erg 
hetzelfde". Juist daardoor ben jij iemand die me laat zien dat dingen ook heel anders kunnen, en dat is soms echt nodig!

Lieve Opa Wim, mijn opa is 90 en hij maakt mijn promotie mee! Ik denk er vaak aan hoe bijzonder dat is (oma had het ook mooi gevonden he). Ik heb ontzettend veel respect voor hoe je de belangrijke dingen in het leven weet te benoemen en hoop dat ik dat de rest van mijn leven ook zal doen.

Papa en mama, mijn mentor op de middelbare school zei al "jouw ouders staan altijd achter je he?", waarop ik natuurlijk alleen maar "ja" kon antwoorden. Dank jullie wel voor de oneindige steun (op een paar scherp geformuleerde sinterklaasgedichten na). Natuurlijk ook voor de gezelligheid, het thuiskomen, post-wandel diners in Limburg, die sinterklaasgedichten dus, willekeurige schapen-foto's van over de hele wereld... en zo veel meer. Een beroemde schrijver schreef "There is no magic on earth strong enough to wipe out the legacies of one's parents" en dat is maar goed ook. 
Curriculum vitae 



\section{Curriculum vitae}

Martine Moossdorff was born on May 21 1988 in Utrecht, The Netherlands. After graduating from secondary school at the Christelijk Gymnasium Utrecht in the summer of 2006, she started medical school at Maastricht University. During the Bachelor's programme, she successfully participated in the Honours Programme International Health. Furthermore, she took one year to participate in the executive committee of her student association (S.V. KoKo, Maastricht) and completed a minor in Globalization and Diversity at the faculty of Arts and Culture, as well as a minor in Health Law at the faculty of Law of Maastricht University. She received her Bachelor's degree in Medicine on December 31, 2010. Afterwards, she proceeded to the Master's programme and graduated cum laude (with distinction) from Medical School on August $31^{\text {st }}, 2013$. During the final year of medical school, she wrote a master thesis on endpoints in breast cancer research, which is the foundation on which this $\mathrm{PhD}$ project was built. For this master thesis, she was awarded the Student Prize for Master Theses of the Faculty of Health, Medicine and Life Sciences. After graduating, she was employed as a PhD candidate at the department of Surgery at Maastricht University/Maastricht University Medical Center from October 2013 until June 2016, with the breast cancer surgery research group of Dr. M.L. (Marjolein) Smidt and under supervision of Prof. L.P.S. (Laurents) Stassen. Martine was awarded the GROW Best Oncology Paper Award of 2014 for Chapter 2 of this thesis. From April 2016 until June 2016, she worked as a guest research fellow at Dana Farber/Brigham and Women's Cancer Center in Boston, Massachusetts (USA), in Dr. T.A. (Tari) King's research group.

Since July 2016, she is employed as a surgical resident at the department of Surgery at Zuyderland Medical Center in Sittard and Heerlen, The Netherlands. As of July $1^{\text {st }}, 2017$, she has started her formal surgical training. 

List of publications 



\section{List of publications}

Moossdorff M, Van Roozendaal LM, Strobbe LJA, Aebi S, Cameron DA, Dixon JM, Giuliano AE, Haffty BG, Hickey BE, Hudis CA, Klimberg S, Koczwara B, Kühn T, Lippman ME, Lucci A, Piccart MJ, Smith BD, Tjan-Heijnen VCG, Van de Velde CJH, Van Zee KJ, Vermorken JB, Viale G, Voogd AC, Wapnir IL, White J, Smidt ML. Maastricht Delphi Consensus on event definitions for classification of recurrence in breast cancer research. Journal of the National Cancer Institute 2014;106(12).

Moossdorff M, Van Roozendaal LM, Schipper R, Strobbe LJA, Voogd AC, Tjan-Heijnen VCG, Smidt ML. Inconsistent selection and definition of local and regional endpoints in breast cancer research. British Journal of Surgery 2014;101(13):1657-1665.

Moossdorff M, Vugts G, Maaskant-Braat AJG, Strobbe LJA, Voogd AC, Smidt ML, Nieuwenhuizen GAP. Contralateral lymph node recurrence in breast cancer: Regional event rather than distant metastatic disease. A systematic review of the literature. European Journal of Surgical Oncology 2015;41(9):1128-1136.

Schipper RJ, Moossdorff M, Nelemans PJ, Nieuwenhuijzen GA, de Vries B, Strobbe LJ, Roumen RM, van den Berkmortel F, Tjan-Heijnen VC, Beets-Tan RG, Lobbes MB, Smidt ML. A Model to Predict Pathologic Complete Response of Axillary Lymph Nodes to Neoadjuvant Chemo(Immuno)Therapy in Patients With Clinically Node-Positive Breast Cancer. Clin Breast Cancer 2014;14(5):315-322.

Van Nijnatten TJA, Moossdorff M, de Munck L, Goorts B, Vane MLG, Keymeulen KBMI, Beets-Tan RGH, Lobbes MBI, Smidt ML. TNM Classification and the need for revision of pN3a breast cancer. Eur J Cancer 2017;79:23-30.

Van Nijnatten TJA, Simons JM, Moossdorff M, De Munck L, Lobbes MB, Van der Pol CC, Koppert LB, Luiten EJ, Smidt ML. Prognosis of residual axillary disease after neoadjuvant chemotherapy in clinically node-positive breast cancer patients: isolated tumor cells and micrometastases carry a better prognosis than macrometastases. Breast Cancer Res Treat 2017;163(1):159-166.

Goorts B, Van Nijnatten TJ, De Munck L, Moossdorff M, Heuts EM, De Boer M, Lobbes $M B$, Smidt ML. Clinical tumor stage is the most important predictor of pathological complete response rate after neoadjuvant chemotherapy in breast cancer patients. Breast Cancer Res Treat 2017;163(1):83-91. 
Habraken V, Nijnatten TJ van, Munck L de, Moossdorff M, Heuts EM, Lobbes MB, Smidt $\mathrm{ML}$. Does the TNM classification of solitary internal mammary lymph node metastases in breast cancer still apply? Breast Cancer Res Treat 2017;161(3):483-389.

Schipper RJ, Moossdorff M, Beets-Tan RGH, Smidt ML, Lobbes MBI. Noninvasive nodal restaging in clinically node positive breast cancer patients after neoadjuvant systemic therapy: a systematic review. European journal of radiology 2015;84(1):41-47. IF 2.304

Kuijs VJL, Moossdorff M, Schipper RJ, Beets-Tan RGH, Heuts EM, Keymeulen KB, Smidt $\mathrm{ML}$, Lobbes $\mathrm{MBI}$. The role of $\mathrm{MRI}$ in axillary lymph node imaging in breast cancer: a systematic review. Insights into Imaging. 2015;6:203-215.

Bosmans JW, Moossdorff M, Al-Taher M, Beek L van, Derikx JP, Bouvy ND. International consensus statement regarding the use of animal models for research on anastomoses in the lower gastrointestinal tract. Int J Colorectal Dis. 2016;31(5):1021-30.

Van Roozendaal LM, Schipper R, Moossdorff M, Strobbe LJA, Smidt ML. [Even goed, met minder: Behandeling van de oksel bij mammacarcinoompatiënten]. Kanker Breed 2013; 5(2):3-7. 



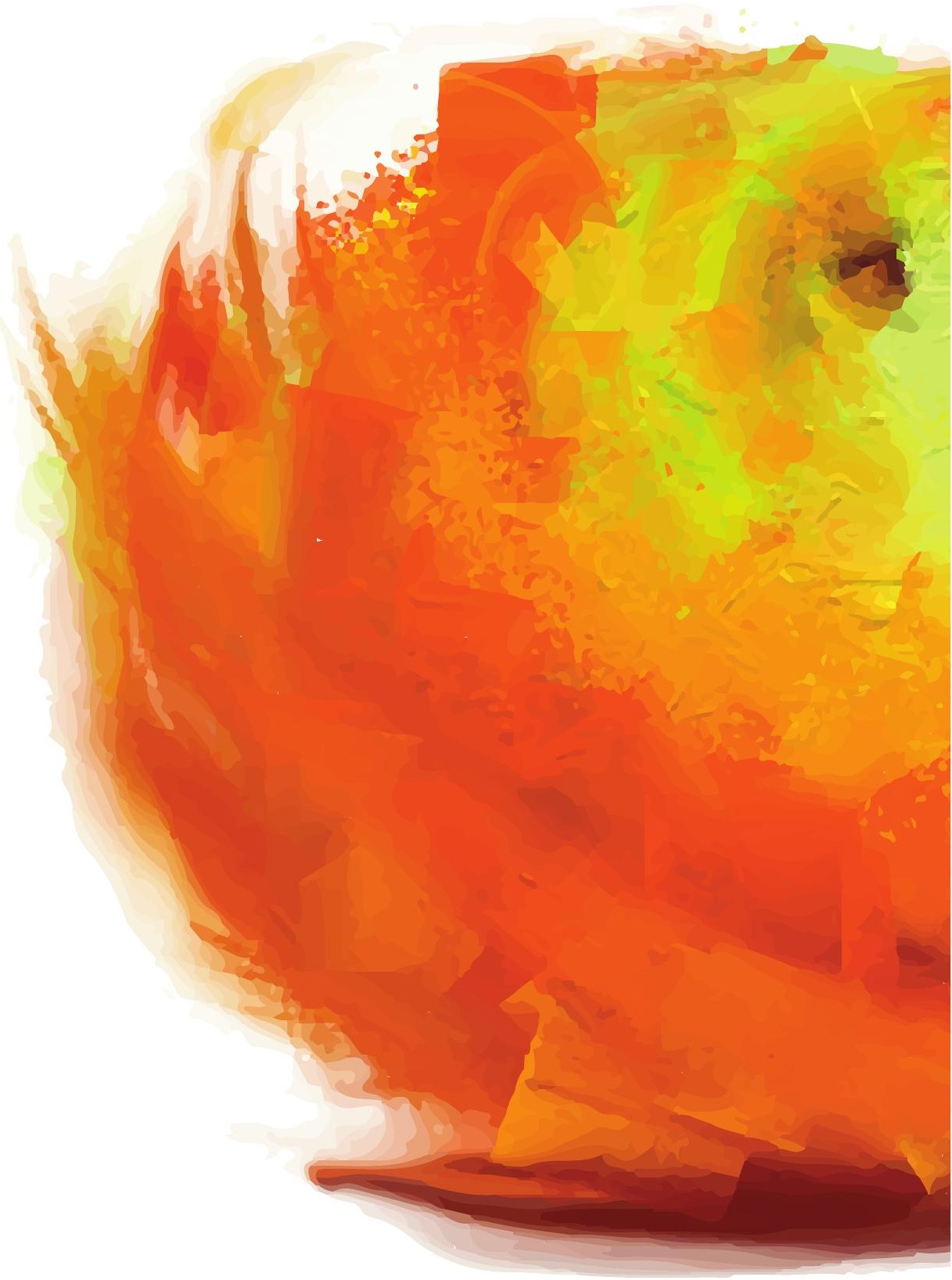

